\title{
WestVirginiaUniversity
}

THE RESEARCH REPOSITORY @ WVU

Graduate Theses, Dissertations, and Problem Reports

2008

\section{Non-apoptotic roles of caspase-8 and caspase-2}

Brooke M. Helfer

West Virginia University

Follow this and additional works at: https://researchrepository.wvu.edu/etd

\section{Recommended Citation}

Helfer, Brooke M., "Non-apoptotic roles of caspase-8 and caspase-2" (2008). Graduate Theses,

Dissertations, and Problem Reports. 4380.

https://researchrepository.wvu.edu/etd/4380

This Dissertation is protected by copyright and/or related rights. It has been brought to you by the The Research Repository @ WVU with permission from the rights-holder(s). You are free to use this Dissertation in any way that is permitted by the copyright and related rights legislation that applies to your use. For other uses you must obtain permission from the rights-holder(s) directly, unless additional rights are indicated by a Creative Commons license in the record and/ or on the work itself. This Dissertation has been accepted for inclusion in WVU Graduate Theses, Dissertations, and Problem Reports collection by an authorized administrator of The Research Repository @ WVU.

For more information, please contact researchrepository@mail.wvu.edu. 
NON-APOPTOTIC ROLES OF CASPASE-8 AND CASPASE-2

$$
\text { by }
$$

Brooke M Helfer
Dissertation submitted to the School of Medicine at West Virginia University
in partial fulfillment of the requirements
for the degree of

Doctor of Philosophy in Cancer Cell Biology

Laura Gibson, PhD, Committee Chairperson

Linda Vona-Davis, PhD

Elena Pugacheva PhD

Daniel Flynn PhD

Scott Weed PhD

Steven Frisch PhD, mentor

Morgantown, West Virginia

2008

Keywords: Caspase-8, Caspase-2, non-apoptotic, adhesion, migration, cytoskeleton 


\section{Abstract \\ NON-APOPTOTIC ROLES OF CASPASE-8 AND CASPASE-2 \\ by Brooke M Helfer}

Caspases are proteolytic enzymes involved in committing apoptosis, but through the studies of knockout phenotypes in mice and Drosophila, it has been speculated that caspases might possess additional non-apoptotic functions.

We have found non-apoptotic functions for caspase-8 in both normal and tumor cell lines. Specifically, we found that caspase-8 promotes cell migration, adhesion, and Rac activation. Subsequently, we also found that caspase-8 interacts with the p85 subunit of PI3K. Accompanying stimulation of motility with epidermal growth factor the phosphorylation of caspase-8 on tyrosine-380 is induced and this phosphorylation allows for p85 interaction, cell migration, adhesion, and Rac activation. Caspase-8 also promoted EGF-induced Erk activation, stimulating cell migration.

Non-apoptotic functions also exist for caspase-2. We demonstrate that caspase-2 promotes cell-matrix adhesion, focal contact formation and FAK phosphorylation. Caspase 2 also mediates stress fiber dissolution in response to protein kinase-C activation. Insights into the mechanism whereby caspase 2 influences cytoskeletal processes with emphasis on Rho/ROCK/LIMK/cofilin and Rho/ROCK/MLC pathways are discussed.

These findings demonstrate a non-apoptotic function of a caspase involving signaling protein interactions rather than proteolysis. This is also the first report that caspase-2 and caspase-8 may mediate cytoskeletal functions independent of cell death. 


\section{Acknowledgments}

I decided in Mrs. Shank's $10^{\text {th }}$ grade biology class that I was going to become a scientist. We had read The Hot Zone and I was hooked. Not long ago, I was able to tell Mrs. Shank that her class really shaped the person I had become.

In college the guidance of Dr Alice Lee and Susan Pietropaolo taught me strength and a deeper appreciation of research. I had the opportunity to work directly with both of these women and truly gained from the experience.

In graduate school, my advisor Dr Steven Frisch pushed me to meet my full potential and became a friend in the process. He and many others in the Health Science Center became mentors in life as well as science.

Vicki, Stephanie, Jamie, Rachel, Amy and Andrea have been amazing friends, study partners, and there to bounce many an idea off of!

Finally I would like to acknowledge my family, specifically my parents, Ken and Delila, my sister Nikki, Grannie Polly, Pappy Brooks, and my Godparents Aunt Nene and Uncle Sonny - their unwavering love, support, and encouragement of my dreams cannot be put into words.

I couldn't have done it without all of you!! 


\section{Table of Contents}

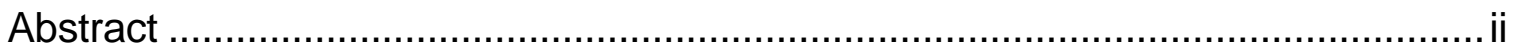

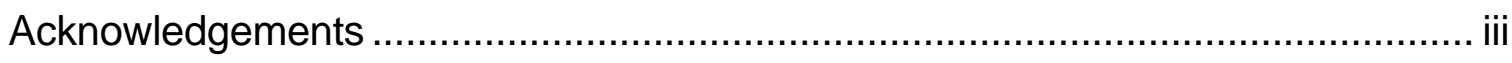

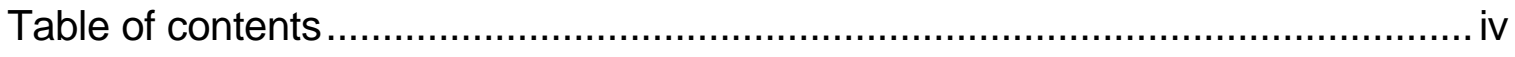

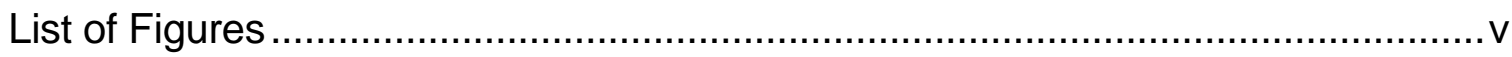

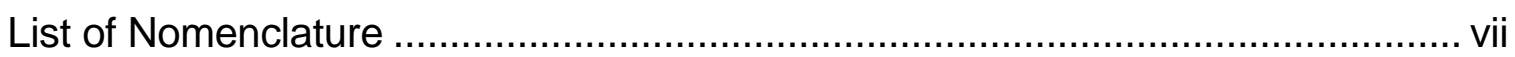

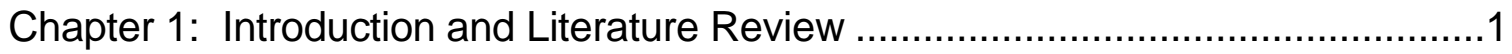

1. Migration and its role in cancer promotion ......................................

2. Apoptotic functions of caspase 2 and 8 ....................................10

3. Non-apoptotic roles of caspases ..............................................13

Chapter 2: Caspase-8 promotes cellular migration and adhesion.......................48

Chapter 3: Pro-caspase-8 promotes cellular migration and adhesion through interaction with the p85 subunit of $\mathrm{PI} 3 \mathrm{~K}$

Chapter 4: Caspase-2 promotes cellular adhesion, FAK activation, and the breakdown of actin stress fibers.

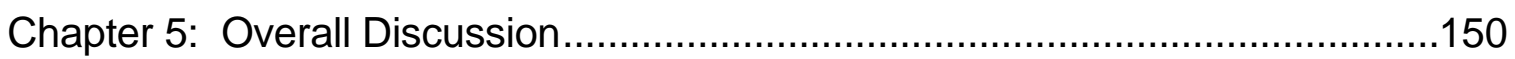

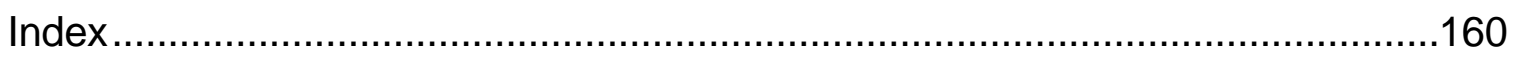

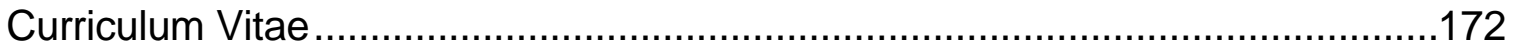




\section{LIST OF FIGURES}

Chapter 1. Introduction and Literature Review:

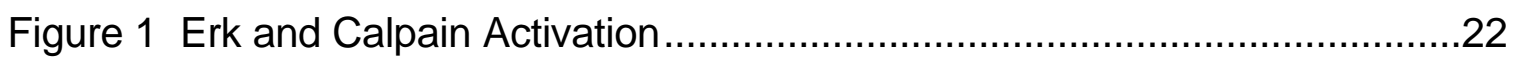

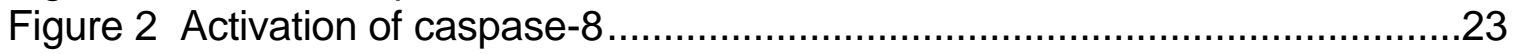

Chapter 2. Caspase-8 promotes cellular migration and adhesion

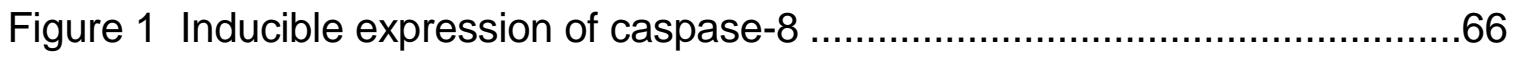

Figure 2 Caspase-8 promotes cell motility ................................................. 65-69

Figure 3 Caspase-8 and FASL promote motility \& invasion ......................... 70-72

Figure 4 Caspase-8 promotes Rac activity ....................................................

Figure 5 Caspase-8 affects cellular processes downstream

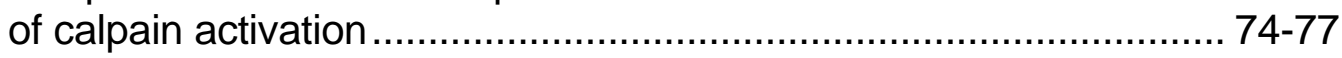

Figure 6 Caspase-8 promotes Erk activation................................................78

Figure 7 Proposed pathway for how caspase-8 is influencing

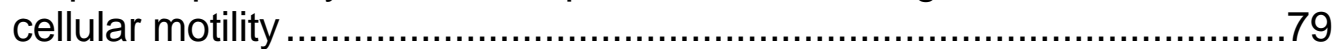

Chapter 3. Pro-Caspase-8 promotes cellular migration and adhesion through interaction with the p85 subunit of PI3K

Figure 1 Caspase-8 expression is maintained in human tumors. 102

Figure 2 Caspase-8 enhancement of cell adhesion/motility is dependent on tyrosine-380 but independent of catalytic activity or autoproteolytic processing 103

Figure 3 Caspase-8 interacts with p85 104-107

Figure 4 Caspase-8-p85 interaction contributes to Rac activation but does not enhance Akt activation 108-109

Figure 5 Caspase-8 relocalizes to cell periphery upon EGF treatment

Figure 6 D285H, a naturally occurring SNP does not influence cellular motility or adhesion

Figure 7 D302H, a mutation adjacent to a YXXM motif influences cellular motility and adhesion.

Figure 8 Caspase-8 is involved in an apoptosis motility switch

Chapter 4. Caspase-2 promotes cellular adhesion, FAK activation, and the breakdown of actin stress fibers

Figure 1 Caspase-2 promotes cellular adhesion 136

Figure 2 Caspase-2 promotes focal contact formation 
Figure 3 Caspase-2 promotes FAK phosphorylation at Y397 upon cellular adhesion

Figure 4 Caspase-2 promotes the loss of stress fibers upon

PMA/TPA treatment

Figure 5 Caspase-2 expression may alter ROCKII cleavage

Figure 6 Cofilin phosphorylation is not altered by caspase-2 expression

Figure 7 Phosphorylation of MLC does not does not differ with caspase-2 expression upon PMA treatment

Figure 8 Tumor tissues do not lose caspase-2 expression 


\section{LIST OF NOMENCLATURE}

\begin{tabular}{ll}
$\boldsymbol{\alpha}$ & alpha \\
ATP & adenosine triphosphate \\
$\boldsymbol{\beta}$ & beta \\
$\mathbf{C}$ & Celcius \\
$\boldsymbol{\mu}$ & micro \\
DAG & diacylglycerol \\
DD & Death Domain \\
DED & Death effector domain \\
DISC & Death-inducing signaling complex \\
DMEM & Dulbecco's modified Eagle's medium \\
DMSO & Dimethyl Sulfoxide \\
EGF & Epidermal growth factor \\
EGFR & Epidermal growth factor receptor \\
Erk & Extracellular signal-regulated kinase \\
FADD & Fas-associated death domain \\
FAK & Focal adhesion kinase \\
FBS & Fetal bovine serum \\
GFP & Green fluorescent protein \\
HBSS & Hank's balanced salt solution \\
kDa & kiloDalton \\
LPA & lysophosphatidic acid \\
\hline
\end{tabular}




\begin{tabular}{ll} 
MAPK & Mitogen-activated protein kinase \\
MEF & Mouse embryo fibroblast \\
MLC & Myosin light chain \\
MLCK & Myosin light chain kinase \\
MMP & Matrix metalloproteinase \\
NF-KB & Nuclear factor-kappa B \\
PARP & Poly ADP ribose polymerase \\
PBS & Phosphate buffered saline \\
PIDD & p53-induced protein with death domain \\
PI3K & phosphoinositide-3 kinase \\
PIP2 & phopshatidylinositol bisphosphate \\
PIP3 & phosphatidylinositol 3, 4 ,5-triphosphate \\
PKC & Protein kinase C \\
PMA & phorbol 12-myristate 13-acetate \\
ROCK & Rho kinase \\
SREBP & Sterol response element binding protein \\
TPA & phorbol 12-myristate 13-acetate \\
TRAIL & Tumor necrosis-related apoptosis-inducing ligand \\
WT & Wild type \\
\hline
\end{tabular}




\section{Chapter 1}

\section{Introduction and Literature Review}

1. Migration and its role in cancer promotion

Types of cell migration

Role of calpains in cell migration

Erk activation in motility and calpain activation

$\mathrm{PI} 3 \mathrm{~K}$ signaling in cellular migration

2. Caspases 2 and 8 in apoptosis

3. Non-apoptotic roles of caspases

Knockout caspase studies

Non-apoptotic functions: Low/basal levels of caspase activity

Non-apoptotic functions: Compartmentalized caspase activity

Other non-apoptotic functions of caspases 


\section{Migration and its role in cancer promotion}

Cell migration is a critical component in cancer progression. Patients with cancer more often die from metastases than from their primary tumor [1]. Gene expression profiles demonstrate that genes associated with cell motility are often increased in carcinoma cells $[2,3]$. Aspects of cell migration and the pathways that control this process directly affect the ability of cells to invade and metastasize [4]. Summarized here are some of the pathways that are integral in the process of cell migration.

\subsection{Types of cell migration}

Cell migration can be broken down into three main types of migration: amoeboid, mesenchymal, and collective. The first two types of migration, amoeboid and mesencymal are types of single cell migration that differ in how they move both phenotypically and mechanistically. The third type of cell migration, collective migration, involves cells moving in groups, connected by cell-cell contacts [5].

Amoeboid: Amoeboid migration is a fast form of migration. Interestingly, amoeboid migration lacks integrin association with matrix and involves only weak interactions with any form of substrate $[6,7]$. These cells move via an expansion and contraction, crawling like mechanism and that is mainly driven through cortical actin dynamics signaled by RhoA and ROCK $[8,9]$. Amoeboid migration is particularly useful when traveling through 3D matrices. Cells employing amoeboid like migration, such as neutrophils and lymphocytes, and cancer cells such as small-cell lung and mammary 
carcinomas and lymphomas, travel through matrices by squeezing through the extracellular matrix rather than needing to degrade it [3, 7, 10-12].

Cell migration of tumors often occurs along fibers of the extracellular matrix (ECM). Cells employ amoeboid migration along these fibers, which often merge with blood vessels, where they can then intravasate into the blood stream and extravasate at a secondary location (reviewed in [13]).

Mesenchymal: Mesynchymal and collective migration utilize migration in the most commonly thought of integrin, Rac dependent manner. Mesenchymal migration occurs via the 4 step process of 1. producing polarized cell projections, 2. stabilizing those projections via focal contacts/adhesions, 3. cell contraction, and 4. rear end detachment resulting in forward cell migration $[14,15]$. This migration is mediated by Rac, Rho and CDC42 promoted cytoskeletal structures. Specifically, Rac and CDC42 promote polarized cellular projections (lamellipodia and filopodia) and Rho mediates cell contractility [16-18].

Lamellipodia projection of these cells has been explored as an important component of cell invasion [19]. Lamellipodia form toward blood vessels in response to chemoattractants such as EGF [19] or those secreted from macrophages [13, 20]. Again, this migration relocates cells to blood vessels where they can enter into the blood stream and spread throughout the body [13]. 
Mesenchymal movement though $3 \mathrm{D}$ matrices is more complex than that seen with amoeboid migration. Mesencymal cells require the digestion or remodeling of ECM, similar to collective migration, in order to move through a matrix, utilizing MMPs [2123].

Collective migration: Collective migration is achieved similarly to mesencymal migration with the difference that cells move as a sheet connected by cell-cell contacts and that the leading cells in the sheet pull connected cells along with them ([24] reviewed in [5]). Collective migration is commonly witnessed in standard scratch wound healing assays, duct formation, sprouting of vasculature, and in invading epithelial clusters (reviewed in [5]). Collective migration and mesenchymal migration will be the means of migration examined throughout the remainder of this document.

\subsection{Role of calpains in cell migration}

Calpain, a protease family that is ubiquitously expressed in cells, has several linkages to cell migration (reviewed in [25-27]). Considering the requirements for cell migration - forming cellular projections, such as lamellipodia, securing those projections with new adhesions, polarization, and rear end detachment - calpain has the ability to impact the majority of these components [28-36]. In light of this, it is not surprising that cells treated with inhibitors of calpain demonstrated decreased cell migration $[33$, 34]. 
Calpain's influence over cell migration is also evident when considering its numerous substrates related to both cellular adhesion and migration. Some of these substrates include talin, vinculin, paxillin, FAK, the EGF receptor, MLCK, and spectrin (reviewed in [26]). Cellular adhesion is both positively and negatively influence by calpain. Cell migration requires both the formation and dissolution of cell adhesions for successful migration. Calpains positively influence adhesions when considering cell spreading. Accordingly, calpain is known to colocalize with the guanine exchange factor aPix. In this context, when cells are treated with a calpain inhibitor, cell spreading and Rac activation are decreased. This effect is able to be rescued by overexpression of aPix suggesting that the interaction of calpain with aPix is able to influence cell spreading [37]. Calpain is also associated with rear end detachment in migrating cells [38]. Cleavage of some of the above listed cellular adhesion related proteins is related with a caplain mediated decrease in cellular adhesion [39]. Calpain proteolysis of paxillin and FAK have also been observed during cell migration [31].

Membrane projections also require the presence of calpain. Calpain knockout mice demonstrate abnormal membrane projections, specifically numerous thin projections with untimely retraction [30]. Furthermore, calpastatin inhibition of calpain inhibited lamellipodia projection and caused abnormal filopodia [35]. In both of these studies, cells demonstrated decreased cell migration. Calpain has also been found necessary for the activation of Rac $[28,29]$, potentially explaining the lack of lamellipodia formation in its absence. 
Calpain-2, based upon it mechanisms for activation, its ability to promote the aspects of cell motility, and the fact that it often displays increased activation or is overexpressed in some tumors [40-42] is an efficient tumor promoter.

\subsection{Erk activation in motility and calpain activation}

The MEKK1/Erk pathway is a pathway of interest because it is involved in the promotion of cell migration, is associated with calpain-2 activation, and is activated upon EGF treatment and cellular adhesion, and is involved in the regulation of transcription factors - it is a multifaceted pathway [43-47]. Here we will consider the impact of Erk activation upon motility, means for Erk activation, and the involvement of calpain activation in the promotion of cell migration.

The Erk pathway influences cellular migration through myosin light chain kinase (MLCK), paxillin, FAK, and calpain signaling (reviewed [46]). As reviewed above,

calpain influences several aspects of cellular migration. Erk may influence these pathways via the phosphorylation induced activation of calpain [48-50]. MLCK is able to be phosphorylated by both MEK1 and Erk [51] and is able to influence cell migration via focal adhesion dynamics and MLCK related cytoskeletal changes [5254]. Erk phosphorylation of MLCK also results in increased MLC phosphorylation and increased cell migration [51]. Erk influence over paxillin regulates adhesion, both formation and turnover by its interaction with FAK [55-57]. The relationship of FAK with Erk in cellular adhesion is somewhat complicated in that adhesion induced FAK activation promotes Erk activation (discussed below), which later results in adhesion 
dissolution though calpain activation by Erk [58] and though Erk's phosphorylation of FAK [57].

Cellular adhesions can influence the activation of MEKK1/Erk via FAK to calpain [59]. There are multiple pathways for the activation of ERK by cell adhesion. First, FAK phosphorylation on Y925 by src is believed to recruit Grb2, and thus activate the classic Ras pathway $[60,61]$. PAK and Raf-1 signaling also need to be taken into account in adhesion induced Erk activation. PAK activation occurs via src and FAK signaling, upon integrin engagement, which then acts upstream of Erk by activation of MEK1 [62]. Erk supported migration is also achieved though calpain proteolysis of FAK for rear end detachment $[46,58]$.

Interestingly, MEKK1 also binds directly to FAK, and may additionally bind directly to ERK [63]. This interaction, when activated by EGF, is able to activate Erk, and thus influence caplain activation and Erk mediated adhesion/motility dynamics [38]. During apoptosis, caspase-8 cleaves MEKK1 resulting in its activation [64]. This interaction may allow MEKK1 to activate Erk and influence cell migration under non-apoptotic circumstances also, an avenue that has yet to be explored.

MEKK1/Erk activation and downstream calpain phosphorylation also occurs via EGF (and other growth factor) stimulated activation of the Ras pathway [49]. This pathway is well established and will only be described briefly. Activation begins with EGFR stimulation and subsequent receptor phosphorylation. Grb2, an adaptor protein, is 
then able to interact with the GEF Sos to activate Ras. Ras then activates Raf (MAPKKK), which then results in MAPKK to MAPK (Erk) activation [65-67]. Interestingly, several members of the Ras pathway are also able to be induced by adhesion $[60,61]$ and reciprocally FAK can be activated by interaction with EGF, an interaction able to support cell migration [68]. Several pathways in which MEKK1/Erk/calpain activation occur are summarized in Figure 1. Note that some of these pathways are predicted to be caspase-8-dependent, which has not yet been tested.

\subsection{PI3K signaling in cellular migration}

EGF stimulation is an important component in both cell migration and tumor metastasis. EGFR signals the activation of several migratory pathways including Rac, CDC42, PI3K, and Erk [48, 49, 69-73]. The activation of these pathways occurs not only though activation of their specific pathways by EGF but also though pathway cross talk. Deregulation of EGFR signaling would have the potential to wreak cellular havoc, an example being ErbB2 signaling in mammary tumors [74]. EGFR overexpression and signaling through EGF have been connected to both increased migration and invasion [75-77], including breast cancer with ErbB2 expression [78-80].

The phosphatidylinositol-3-kinase (PI3K) pathway is one aspect of EGFR signaling. $\mathrm{PI} 3 \mathrm{~K}$ is comprised of three subfamilies, class -1 being comprised of p85 regulatory and p110 catalytic subunits. The p85/110 heterodimer is stimulated by EGF and is able to produce the lipid products phosphatidylinositol 3-monophosphate, phosphatidylinositol 
3,4-bisphosphate, and phosphatidylinositol 3,4,5-triphosphate (reviewed in [81, 82]). These lipid products, especially phosphatidylinositol 3,4,5-triphosphate (PIP3), are able to influence cell migration $[83,84]$. Chemoattractant stimulation of PI3K at the leading edge is one example of how PI3K/PIP3 influences cell migration. PIP3 is associated with the activation of Rac GEFs to mediate Rac activation (reviewed in [85, 86]) and thus cellular protrusions. This interaction also results in CDC42 by aPix and Rho/ROCK cytoskeletal dynamics [86]. Through these processes, PI3K is able to influence the signaling between Rac, Rho, and CDC42, a pathway associated with coordination of lamellipodia projection (Rac), filopodia projection (CDC42), and Rho mediated adhesion and stress fiber modification (reviewed [15]).

PI3K has the ability to activate and be activated by Ras [87-90]. Interaction of PI3K with EGFR [91] is able to mediate activation of the Ras pathway [92]. Through this interaction $\mathrm{PI} 3 \mathrm{~K}$ is able to affect Erk activation as reviewed above. PI3K is also able to activate Raf-1 through Pak resulting in the Raf $\rightarrow$ MEK $\rightarrow$ Erk progression of activation $[93,94]$. 


\section{Apoptotic functions of caspases 2 and 8}

Apoptosis is an organized way of death that occurs in a step-like process. Apoptosis can be described as occurring in three steps - initiation, commitment and execution $[95,96]$. The area of caspase research with regard to apoptosis is astounding. Due to the purpose of this review, the functions of caspase- 8 and caspase- 2 in apoptosis will be reviewed briefly by emphasizing induction of apoptosis and key substrates of these caspases.

Death receptor signaling complexes are responsible for relaying external apoptosis inducing signals to initiator caspases. The DISC, which is means of activating initiator caspase-8, is composed of the CD95/APO1/Fas surface receptor, TRADD, and FADD. Alternatively, FADD is able to bind to CD95 directly without TRADD as an intermediary. Binding of FasL to CD95 results in assembly of the DISC and FADD mediated dimerization of caspase-8. This complex leads to subsequent caspase-8 activation [97] and induction of apoptosis. Activation of caspase-8 occurs via a two step cleavage process. First, the linker region between the $\mathrm{p} 18$ and $\mathrm{p} 10$ subunits of caspase-8 is cleaved at D374 and D384. The second cleavage event is at D216 releasing a mature caspase-8 tetramer from the DISC into the cytoplasm [98] (Figure 2). An interesting dynamic in this process is c-FLIP. c-FLIP can inhibit apoptosis when recruited to a DISC because it competes against caspase-8 recruitment. On the other hand, non-DISC associated caspase-8 is activated by herterodimerization with c-FLIP $[99,100]$. 
Caspase-8 is able to induce MOMP by cleaving Bid protein [101] as well as directly activate caspase-3 and caspase-7 [102], which are "executioner caspases" (ie caspases that cleave critical survival substrates) and plectin [103]; other substrates and binding partners have been proposed as well (hprd.org). Caspase-8 cleavage of plectin may be relevant in the induction of anoikis since plectin is a cytoskeletal anchoring protein. Plectin cleavage by caspase- 8 also is responsible in part for apoptotic reorganization of the cytoskeleton since plectin is a crosslinking protein of the cytoskeleton filament systems [103].

Caspase- 2 activation is also able to be achieved in theory via a death receptor signaling complex, but its main function is in DNA damage-induced apoptosis Caspase-2 is found in a complex with the DISC complex, and is able to be activated by it [104], but is not required for FAS-induced apoptosis [105]. Caspase-2 is able to bind to both CD95 and TNF-R1 via RIP mediated RAIDD binding to caspase-2 through their CARD domains $[106,107]$. Caspase-2 is also recruited to the PIDDosome, through the interaction of its caspase recruitment domain (CARD) with RAIDD [108]. Caspase 2 has been implicated in at least four apoptotic responses: i. heat shock response [109] ii. "mitotic catastrophe", that is cell death induced when mitotic chromosome segregation fails due to improper kinetochore attachment or chromosome damage. Mitotic catastrophe involves activating caspase 2 upstream of mitochondrial outer membrane permeabilization (MOMP) $[110,111]$ iii. DNA damage responses [108, 112-120] iv. Cytoskeletal disruption induced apoptosis [121]. 
Caspase-2 has only a few known substrates. Substrates of note are golgin-160 and Bid. Caspase-2 cleavage of Bid is associated with the mitochondrial driven aspect of apoptosis, of which caspase-2 is thought to be both upstream and downstream of the mitochondria (reviewed in [122]). Bid cleavage by caspase-2 at the mitochondria is responsible for cytochrome $\mathrm{c}$ release and activation of Apaf-1, which further activates caspase- 9 and perpetuates the caspase cascade [123]. Golgin-160 cleavage also influences the apoptotic response in that its cleavage by caspase-2 allows its futher processing by other caspases and result in the disassembly of the golgi apparatus [124]. 


\section{Non-apoptotic functions of caspases}

Caspases, for the most part have been viewed as a cell's death machinery. Recently, functions are emerging for caspases in healthy cells as well. Since caspases are present in cells at all times, it is not surprising that they have functions beyond cell death. The development of these roles often begins with studies of caspases in knock out MEFs and in studies of homologous caspases in other animals. Elucidation of non-apoptotic roles possessed by caspases also produces several trends, these themes and theories are the topics considered here.

\subsection{Caspase knockout animal studies}

The study of caspases has come a long way over the past 15 years. Of note are studies done in both knock out animals and the study of caspase homologs in other animals such as Drosophila (reviewed below). The reasoning that caspases are vital in both life and death begin in these contexts. Caspases 3, 8, and 9 are essential for development and also integral in apoptosis. While other caspases are not embryonic lethal, there are factors in the development of these animals that also suggest roles in addition to apoptosis (reviewed in [125]).

The knock out of initiator caspases 2, 8, and 9 have varying results. Caspases 8 and 9 are lethal when their expression is knocked out. Caspase-8 embyos are E11 embryonic lethal due to the inability to form a functional circulatory system $[125,126]$. More specifically, the conditional knockout of caspase-8 in epithelial cells showed a 
both circulatory failure and lack of neural tube closure [127]. Other conditional knockouts influenced cell differentiation and proliferation (discussed below). Caspase 9 knock outs were perinatally lethal. Most mice died either before or shortly after birth via brain abnormalities; however some were able to survive and developed normally $[125,128,129]$. Contradictory to the idea that initiator caspases are essential for development, knocking out caspase-2 expression does not appear to have much of an effect on developing mice. Female mice show an accumulation of oocytes [130] as their only phenotypic abnormality. This is somewhat surprising given the dependence on the other initiator caspases, 8 and 9, in development and on caspase-2's structural similarity to caspase-9.

Caspase-3 knockouts are lethal as well [131]. Caspase-3 lethality is due to excessive brain tissue expansion, similar to caspase-9. This finding is not surprising given later studies that determine an essential role of caspase-3 and -9 in the differentiation of many cell types (reviewed below), a factor that is undoubtedly an additional aspect in their lethality. Caspase- 3 is one of the three caspases that are thought of as executioner caspases. Caspases 6 and 7 are also executioner caspases, however their knockouts have not been published exclusively, they have been reported as unpublished data, suggesting that caspase- 6 is the only executioner caspase when knocked out results in normal development [125].

Other mouse caspase knockouts include inflammation related caspases: caspase-1, caspase-11, and caspase-12. While these animals develop normally they 
demonstrate deficient apoptosis and often defects in IL-1 $\alpha$ and IL-1 $\beta$ production [132], roles that are not surprising given their apoptotic context.

\subsection{Non-apoptotic functions: Low/basal levels of caspase activity}

In some cases caspases serving non-apoptotic functions exhibit hallmarks of apoptosis but yet do not cause cell death, termed "incomplete apoptosis" [133]. This theme is present across several caspases but is most common with caspase- 3 , a caspase whose activation was once viewed as a point of no return.

Caspase- 3 is involved in the differentiation of lens epithelial cells into lens fibers. The activation of caspase-3 is required for this to occur and results in TUNEL-positive nuclei and PARP cleavage. Addition of a caspase-inhibitor prevents these processes from occurring suggesting further that caspase-3 activation is required [134]. Caspase-3 is also accredited in erythroblast maturation/differentiation. Activation of caspase- 3 occurs via the mitochondrial pathway and cleaves lamin B, an important step in the dissolution of the nuclei $[135,136]$. Interestingly, both caspase- 3 and 9 are associated with compartmentalized monocyte to macrophage differentiation. This activation is present in monocyte to macrophage differentiation but not in monocyte to dendritic cell differentiation. This activation is also in the absence of PARP cleavage. This is unique because PARP cleavage, a hallmark of apoptosis, does not occur but cytochrome-c release and Acinus activation [137], and thus can be characterized as both apoptosis independent and exhibiting "incomplete apoptosis". 
Caspase-14 is also involved in differentiation that results in cell enucleation. Caspase-14 expression is mainly believed to occur in the epidermis, however may also be expressed elsewhere in the body $[138,139]$. Caspase- 14 is associated with human keratinocyte differentiation. While the mechanism in which caspase-14 is contributing to this differentiation is unclear, it is believed to be catalytically active and to be responsible for enucleation of keratinocytes in skin barrier formation $[133,140-$ 142].

Caspases 1 and 3 are associated with skeletal muscle differentiation [143, 144]. While the activity of both caspases is connected to differentiation, the means in which they influence skeletal muscle differentiation are unique. Caspase-1 degrades calpasatin (calpain specific inhibitor) during myoblast differentiation. Calpain activity is required for myoblast fusion into multinucleate fibers. Caspase- 1 is responsible for a decrease in calpastatin, allowing calpain activation, resulting in calpain degradation of proteins to allow for the fusion of myoblasts [143]. Caspase-3 is believed to influence skeletal muscle differentiation via a Mammalian Sterile Twenty-like kinase (MST1) related mechanism. One mean for the activation of MST1 is through cleavage by capsase-3 [145]. Caspase-3 knockout mice have defective skeletal muscle differentiation [132], defects in these myoblasts is restored with active MST1 transfection [144].

Caspase-3, -9, and -1 are also involved in brain development and neuronal differentiation. Caspase-1 involvement in neuronal differentiation is similar to its role 
in myoblast differentiation in that its activity is coupled with calpastatin inhibition and calpain activity. Both caspase-1 and calpain are linked to fodrin degradation, a process that occurs in the later stages of differentiation [146]. Caspase- 3 and -9 are also connected to brain development in that they are believed to be associated with neuronal differentiation, migration (discussed below), and in cell death [131, 147-153].

Caspase- 3 and -8 activity is implicated in promotion the migration and invasion of glioblastoma cells [153]. Caspase-3 activity was found both in areas of necrosis and where unrelated to necrosis. The cells with activated caspase- 3 in areas unrelated to necrosis showed no signs of apoptosis. Interestingly, glioblastoma cells treated with broad spectrum caspase inhibitors and zDEVD, a caspase $3 / 7$ inhibitor demonstrated decreased migration in standard scratch assays. Furthermore, this caspase activity is responsible for the cleavage of gelsolin, a motility associated protein [153].

\subsection{Non-apoptotic functions: Compartmentalized caspase activity}

Caspases have various non-apoptotic roles. The different functions listed above are all united in that they involve basal/low levels of active caspases. The non-apoptotic functions considered here involve caspase activity that differs from those already considered in that it is believed to be contained and not distributed throughout the cell.

Caspase-3, and caspase-9 are involved in the formation of platelets. Platelet formation via caspase- 3 and caspase- 9 is similar to the formation of lens fibers in that it is believed to involve PARP cleavage and similar to glioblastoma caspase-3 induced 
migration in that it involves the cleavage of gelsolin [154]. Staining patters of activated caspase- 3 led the authors to believe that compartmentalized activation of caspases resulted in platelet formation. Caspase-2 is also associated with platelets. Endothelial cells when stimulated with thrombin cleave ROCK in the process of producing endothelial microparticles (EMP). This ROCK cleavage is decreased upon caspase-2 inhibition suggesting a role for caspase-2 mediated ROCK cleavage in EMP formation [155]. EMP formation is associated with the aggregation of platelets [156]. Although the localization (ig. compartmentalized) of caspase-2 has not been explored in this context, the activation of caspase- 3 and -9 is believed to be compartmentalized in the formation of platelets.

\subsection{Other non-apoptotic functions}

Several non-apoptotic roles of caspases are demonstrated in Drosophila melanogaster. DRONC, a Drosophila caspase and homolog of caspases 2 and 9, has non-apoptotic functions. DRONC and DIAP1 (Drosophila inhibitor of apoptosis 1) are connected to border cell migration during Drosophila development though interaction of DIAP1 with the Rac pathway [157]. Regulation of this nonapoptotic function is through DmIKKe, an antagonist of DIAP [158, 159]. It is suggested that DmIKKE is a negative regulator of actin polymerization and a promoter of the nonapoptotic functions of DRONC [160]. DmIKKE may not be the only factor influencing DRONC and DIAP1's role in motility. Factors such as Rac, actin, and other actin regulating proteins cannot be ruled out as regulators of these effects [160]. DRONC is also associated with proliferation of cells in the Drosophila wing disc [161]. 
Cell death results in the compensatory proliferation of cells in the disc. DRONC has been found to increase both proliferation and Wingless expression (also believed to contribute to proliferation) [161].

Drosophila caspases DRICE and DRONC activity is also found to be required for sperm differentiation. DRICE, Drosophila caspase-3-like caspase, activation in sperm is responsible for apoptosis like remodeling of the cytoplasm, in that the bulk of the cytoplasm is removed. This activation of caspases in sperm differentiation is brought about by cytochome-c and DRICE activation occurs in the early steps of sperm individualization and is present throughout this process [162, 163]. Furthermore, DARK, DRONC's activating binding adaptor, and HID, a Drosophila death inducer, are all required along with DRONC for DRONC activation in sperm individualization [164]. Given the similarity of Drosophila and human caspases, it will be interesting to see if human caspases possess similar non-apoptotic functions.

Similar to DARK's influence with DRONC in the non-apoptotic functions of Drosophila, CD95 is associated with non-apoptotic functions in humans. CD95 signaling is linked to cellular invasion [165] through NF-kB, Erk, and caspase 8. Erk activation is further connected to caspase-like activity in that its activation has been connected to association with DED and FADD/caspase-8/c-FLIP signaling $[166,167]$. PEA-15 is a death effector domain (DED) containing protein that regulates Fas induced apoptosis [168, 169]. Interestingly, PEA-15 is also able to influence Erk activity through interaction of Erk with its DED [166, 170, 171]. FADD/caspase-8/c-FLIP signaling is 
involved in the activation of ERK though stimulation with TNF [167]. Moreover, the overexpression of any of these components results in further activation of Erk. It is also interesting to note that when stimulated with TNF cells do not need the enzymatic activity of caspase-8 for Erk activation [167].

TNFR1 signaling leads to NF-KB activation [172]. Caspase-8 and caspase-10 are linked to NF-KB activation through RIP and NIK, and requires the activity of IKK. The DEDs of caspase- 8 and -10 are thought to be required for this activation that has antiapoptotic ramifications [173].

CD95, FADD, C-FLIP, and caspase-8 are also involved in aspects of immune function [174-179]. CD95, FADD, c-FLIP, and caspase-8 are implicated in T-cell proliferation and when these components are lacking proliferation is impaired [174, 178, 180, 181]. Caspase-8 activity is required for NF-KB activation, which is required for both T-cell proliferation and activation [182]. c-FLIP has also been connected to activation, NFKB activation, and Erk activation in T-cells [183-186]. Furthermore, c-FLIP is also involved in the development and maturation of T lymphocytes [183].

Finally, Caspase-11 is also associated with the promotion of motility. Caspase-11 though interaction with Aip1 influences cofilin mediated actin depolymerization [187]. This interaction, with its influence over cofilin is one example of a caspase having influence over cytoskeletal dynamics. Another example is the potential interaction of caspase-2 with RhoB in stress fiber dynamics [188]. While the interaction of caspase- 
2 with RhoB is under apoptotic circumstances, the interaction could also have relevance under non-apoptotic conditions.

\section{Closing Remarks}

Understanding the non-apoptotic roles of caspases is a growing area of research. Of the 14 human caspases $2,3,8,9$, and 11 have been linked to various functions including development, T-cell maturation, proliferation, and/or differentiation (reviewed in $[133,177,189])$. Interestingly, caspases 3,8 , and 9 are also essential for embryonic development. Caspases have also been found to have varying expression levels in both cancer and normal cell types [190-192]. When this information is taken together, it is probable that there are still non-apoptotic roles for caspases that have yet to be explored and that these non-apoptotic functions could have important ramifications in cancer therapies. 


\section{Figure 1}

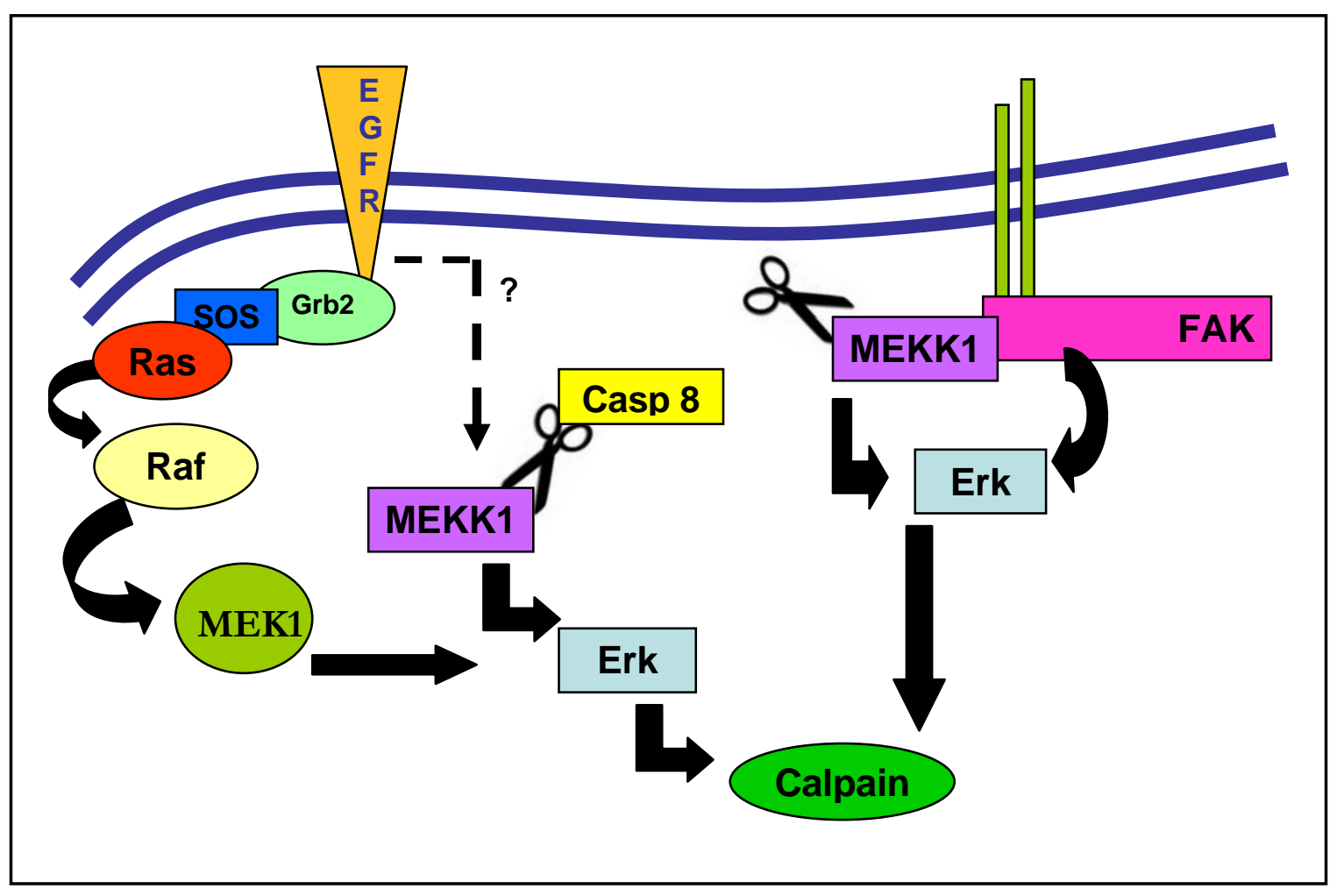

Figure 1: Erk and Calpain Activation. Pathways of Erk and calpain activation, reviewed in section 1.3. The pathway from EGFR through Ras to Erk and calpain activation is well established, however, the pathway of activation from EGFR to MEKK1 is not known 


\section{Figure 2}

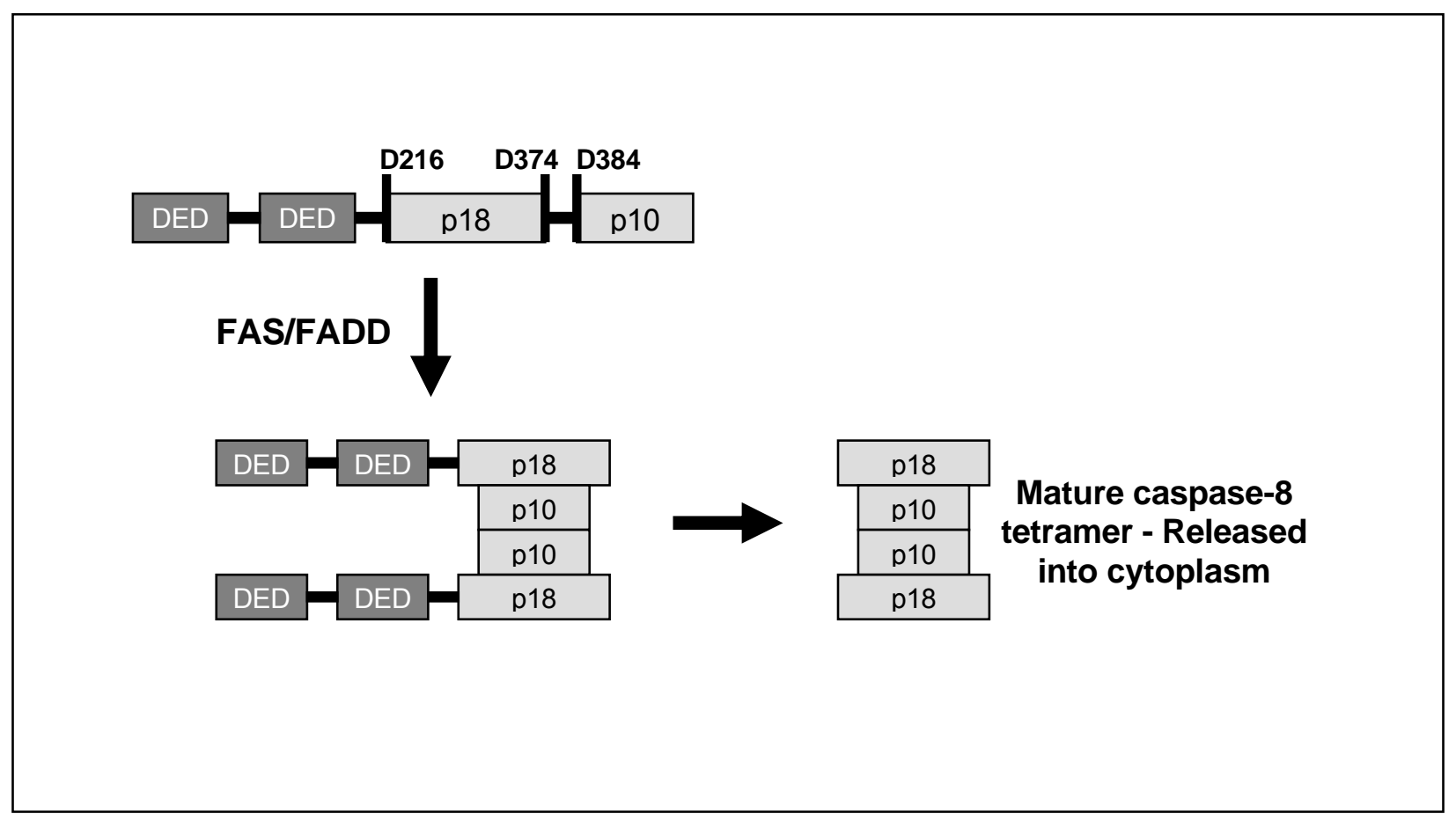

Figure 2: Activation of caspase-8. Caspase-8 dimerizes at the DISC and is autoprocessed by two cleavage events. The first cleavage is at the linker region between p18 and p10 subunits at D374 and D384. The second cleavage event is at D216 which releases the mature caspase-8 tetramer from the DISC into the cytoplasm [98]. 


\section{References}

1. Chambers, A.F., A.C. Groom, and I.C. MacDonald, Metastasis: Dissemination and growth of cancer cells in metastatic sites. Nat Rev Cancer, 2002. 2(8): p. 563.

2. Wang, W., et al., Identification and testing of a gene expression signature of invasive carcinoma cells within primary mammary tumors. Cancer Res, 2004. 64(23): p. 8585-94.

3. Wang, W., et al., Single cell behavior in metastatic primary mammary tumors correlated with gene expression patterns revealed by molecular profiling. Cancer Res, 2002. 62(21): p. 6278-88.

4. Yamaguchi, H., J. Wyckoff, and J. Condeelis, Cell migration in tumors. Curr Opin Cell Biol, 2005.

5. Friedl, P., Prespecification and plasticity: shifting mechanisms of cell migration. Curr Opin Cell Biol, 2004. 16(1): p. 14-23.

6. Fey, P., et al., SadA, a novel adhesion receptor in Dictyostelium. J Cell Biol, 2002. 159(6): p. 1109-19.

7. Friedl, P., S. Borgmann, and E.B. Brocker, Amoeboid leukocyte crawling through extracellular matrix: lessons from the Dictyostelium paradigm of cell movement. J Leukoc Biol, 2001. 70(4): p. 491-509.

8. Smith, A., et al., LFA-1-induced $T$ cell migration on ICAM-1 involves regulation of MLCK-mediated attachment and ROCK-dependent detachment. J Cell Sci, 2003. 116(Pt 15): p. 3123-33. 
9. Worthylake, R.A., et al., RhoA is required for monocyte tail retraction during transendothelial migration. J Cell Biol, 2001. 154(1): p. 147-60.

10. Friedl, P. and K. Wolf, Tumour-cell invasion and migration: diversity and escape mechanisms. Nat Rev Cancer, 2003. 3(5): p. 362-74.

11. Verschueren, H., P. De Baetselier, and J. Bereiter-Hahn, Dynamic morphology of metastatic mouse T-lymphoma cells invading through monolayers of 10T1/2 cells. Cell Motil Cytoskeleton, 1991. 20(3): p. 203-14.

12. Wolf, K., et al., Amoeboid shape change and contact guidance: T-lymphocyte crawling through fibrillar collagen is independent of matrix remodeling by MMPs and other proteases. Blood, 2003. 102(9): p. 3262-9.

13. Condeelis, J. and J.E. Segall, Intravital imaging of cell movement in tumours. Nat Rev Cancer, 2003. 3(12): p. 921-30.

14. Lauffenburger, D.A., Cell motility. Making connections count. Nature, 1996. 383(6599): p. 390-1.

15. Lauffenburger, D.A. and A.F. Horwitz, Cell migration: a physically integrated molecular process. Cell, 1996. 84(3): p. 359-69.

16. Nobes, C.D. and A. Hall, Rho GTPases control polarity, protrusion, and adhesion during cell movement. J Cell Biol, 1999. 144(6): p. 1235-44.

17. Clark, E.A., et al., Integrin-mediated signals regulated by members of the rho family of GTPases. J Cell Biol, 1998. 142(2): p. 573-86. 
18. Keely, P.J., et al., Cdc42 and Rac1 induce integrin-mediated cell motility and invasiveness through PI(3)K. Nature, 1997. 390(6660): p. 632-6.

19. Condeelis, J.S., et al., Lamellipodia in invasion. Semin Cancer Biol, 2001. 11(2): p. 119-28.

20. Lin, E.Y., et al., Colony-stimulating factor 1 promotes progression of mammary tumors to malignancy. J Exp Med, 2001. 193(6): p. 727-40.

21. Kerkela, E. and U. Saarialho-Kere, Matrix metalloproteinases in tumor progression: focus on basal and squamous cell skin cancer. Exp Dermatol, 2003. 12(2): p. 109-25.

22. Nabeshima, K., et al., Front-cell-specific expression of membrane-type 1 matrix metalloproteinase and gelatinase A during cohort migration of colon carcinoma cells induced by hepatocyte growth factor/scatter factor. Cancer Res, 2000. 60(13): p. 3364-9.

23. Wolf, K., et al., Compensation mechanism in tumor cell migration: mesenchymal-amoeboid transition after blocking of pericellular proteolysis. $\mathrm{J}$ Cell Biol, 2003. 160(2): p. 267-77.

24. Farooqui, R. and G. Fenteany, Multiple rows of cells behind an epithelial wound edge extend cryptic lamellipodia to collectively drive cell-sheet movement. J Cell Sci, 2005. 118(Pt 1): p. 51-63.

25. Franco, S.J. and A. Huttenlocher, Regulating cell migration: calpains make the cut. J Cell Sci, 2005. 118(Pt 17): p. 3829-38. 
26. Wells, A., A. Huttenlocher, and D.A. Lauffenburger, Calpain proteases in cell adhesion and motility. Int Rev Cytol, 2005. 245: p. 1-16.

27. Ridley, A.J., et al., Cell migration: integrating signals from front to back. Science, 2003. 302(5651): p. 1704-9.

28. Bialkowska, K., et al., Evidence that beta3 integrin-induced Rac activation involves the calpain-dependent formation of integrin clusters that are distinct from the focal complexes and focal adhesions that form as Rac and RhoA become active. J Cell Biol, 2000. 151(3): p. 685-96.

29. Bialkowska, K., T.C. Saido, and J.E. Fox, SH3 domain of spectrin participates in the activation of Rac in specialized calpain-induced integrin signaling complexes. J Cell Sci, 2005. 118(Pt 2): p. 381-95.

30. Dourdin, N., et al., Reduced cell migration and disruption of the actin cytoskeleton in calpain-deficient embryonic fibroblasts. J Biol Chem, 2001. 276(51): p. 48382-8.

31. Franco, S., B. Perrin, and A. Huttenlocher, Isoform specific function of calpain 2 in regulating membrane protrusion. Exp Cell Res, 2004. 299(1): p. 179-87.

32. Franco, S.J., et al., Calpain-mediated proteolysis of talin regulates adhesion dynamics. Nat Cell Biol, 2004. 6(10): p. 977-83.

33. Huttenlocher, A., et al., Regulation of cell migration by the calcium-dependent protease calpain. J Biol Chem, 1997. 272(52): p. 32719-22. 
34. Palecek, S.P., et al., Physical and biochemical regulation of integrin release during rear detachment of migrating cells. J Cell Sci, 1998. 111 (Pt 7): p. 92940.

35. Potter, D.A., et al., Calpain regulates actin remodeling during cell spreading. J Cell Biol, 1998. 141(3): p. 647-62.

36. Shiraha, H., et al., IP-10 inhibits epidermal growth factor-induced motility by decreasing epidermal growth factor receptor-mediated calpain activity. J Cell Biol, 1999. 146(1): p. 243-54.

37. Rosenberger, G., A. Gal, and K. Kutsche, AlphaPIX associates with calpain 4, the small subunit of calpain, and has a dual role in integrin-mediated cell spreading. J Biol Chem, 2005. 280(8): p. 6879-89.

38. Cuevas, B.D., et al., MEKK1 regulates calpain-dependent proteolysis of focal adhesion proteins for rear-end detachment of migrating fibroblasts. Embo J, 2003. 22(13): p. 3346-55.

39. Glading, A., D.A. Lauffenburger, and A. Wells, Cutting to the chase: calpain proteases in cell motility. Trends Cell Biol, 2002. 12(1): p. 46-54.

40. Braun, C., et al., Expression of calpain I messenger RNA in human renal cell carcinoma: correlation with lymph node metastasis and histological type. Int $\mathrm{J}$ Cancer, 1999. 84(1): p. 6-9.

41. Carragher, N.O., B.D. Fonseca, and M.C. Frame, Calpain activity is generally elevated during transformation but has oncogene-specific biological functions. Neoplasia, 2004. 6(1): p. 53-73. 
42. Wang, Y., et al., Gene-expression profiles to predict distant metastasis of lymph-node-negative primary breast cancer. Lancet, 2005. 365(9460): p. 6719.

43. Pullikuth, A.K. and A.D. Catling, Scaffold mediated regulation of MAPK signaling and cytoskeletal dynamics: a perspective. Cell Signal, 2007. 19(8): p. 1621-32.

44. Katz, M., I. Amit, and Y. Yarden, Regulation of MAPKs by growth factors and receptor tyrosine kinases. Biochim Biophys Acta, 2007. 1773(8): p. 1161-76.

45. Juliano, R.L., et al., Integrin regulation of cell signalling and motility. Biochem Soc Trans, 2004. 32(Pt3): p. 443-6.

46. Huang, C., K. Jacobson, and M.D. Schaller, MAP kinases and cell migration. J Cell Sci, 2004. 117(Pt 20): p. 4619-28.

47. Howe, A.K., A.E. Aplin, and R.L. Juliano, Anchorage-dependent ERK signaling-mechanisms and consequences. Curr Opin Genet Dev, 2002. 12(1): p. 30-5.

48. Glading, A., et al., Epidermal growth factor activates $m$-calpain (calpain II), at least in part, by extracellular signal-regulated kinase-mediated phosphorylation. Mol Cell Biol, 2004. 24(6): p. 2499-512.

49. Glading, A., et al., Epidermal growth factor receptor activation of calpain is required for fibroblast motility and occurs via an ERK/MAP kinase signaling pathway. J Biol Chem, 2000. 275(4): p. 2390-8. 
50. Glading, A., et al., Membrane proximal ERK signaling is required for M-calpain activation downstream of epidermal growth factor receptor signaling. J Biol Chem, 2001. 276(26): p. 23341-8.

51. Klemke, R.L., et al., Regulation of cell motility by mitogen-activated protein kinase. J Cell Biol, 1997. 137(2): p. 481-92.

52. Totsukawa, G., et al., Distinct roles of MLCK and ROCK in the regulation of membrane protrusions and focal adhesion dynamics during cell migration of fibroblasts. J. Cell Biol., 2004. 164(3): p. 427-439.

53. Totsukawa, G., et al., Distinct Roles of ROCK (Rho-kinase) and MLCK in Spatial Regulation of MLC Phosphorylation for Assembly of Stress Fibers and Focal Adhesions in 3T3 Fibroblasts. J. Cell Biol., 2000. 150(4): p. 797-806.

54. Webb, D.J., et al., FAK-Src signalling through paxillin, ERK and MLCK regulates adhesion disassembly. Nat Cell Biol, 2004. 6(2): p. 154-61.

55. Subauste, M.C., et al., Vinculin modulation of paxillin-FAK interactions regulates ERK to control survival and motility. J Cell Biol, 2004. 165(3): p. 37181.

56. Liu, Z.X., et al., Hepatocyte growth factor induces ERK-dependent paxillin phosphorylation and regulates paxillin-focal adhesion kinase association. J Biol Chem, 2002. 277(12): p. 10452-8.

57. Hunger-Glaser, I., et al., PDGF and FGF induce focal adhesion kinase (FAK) phosphorylation at Ser-910: dissociation from Tyr-397 phosphorylation and requirement for ERK activation. J Cell Physiol, 2004. 200(2): p. 213-22. 
58. Carragher, N.O., et al., A novel role for FAK as a protease-targeting adaptor protein: regulation by p42 ERK and Src. Curr Biol, 2003. 13(16): p. 1442-50.

59. Sawhney, R.S., et al., Integrin alpha2-mediated ERK and calpain activation play a critical role in cell adhesion and motility via focal adhesion kinase signaling: identification of a novel signaling pathway. J Biol Chem, 2006. 281(13): p. 8497-510.

60. Schlaepfer, D.D. and T. Hunter, Evidence for in vivo phosphorylation of the Grb2 SH2-domain binding site on focal adhesion kinase by Src-family proteintyrosine kinases. Mol Cell Biol, 1996. 16(10): p. 5623-33.

61. Schlaepfer, D.D. and T. Hunter, Focal adhesion kinase overexpression enhances ras-dependent integrin signaling to ERK2/mitogen-activated protein kinase through interactions with and activation of c-Src. J Biol Chem, 1997. 272(20): p. 13189-95.

62. Slack-Davis, J.K., et al., PAK1 phosphorylation of MEK1 regulates fibronectinstimulated MAPK activation. J Cell Biol, 2003. 162(2): p. 281-91.

63. Yujiri, T., et al., MEK kinase 1 interacts with focal adhesion kinase and regulates insulin receptor substrate-1 expression. J Biol Chem, 2003. 278(6): p. 3846-51.

64. Cardone, M.H., et al., The regulation of anoikis: MEKK-1 activation requires cleavage by caspases. Cell, 1997. 90(2): p. 315-23.

65. Kyriakis, J.M., et al., Raf-1 activates MAP kinase-kinase. Nature, 1992. 358(6385): p. 417-21. 
66. Seger, R. and E.G. Krebs, The MAPK signaling cascade. Faseb J, 1995. 9(9): p. 726-35.

67. Yoon, S. and R. Seger, The extracellular signal-regulated kinase: multiple substrates regulate diverse cellular functions. Growth Factors, 2006. 24(1): p. 21-44.

68. Sieg, D.J., et al., FAK integrates growth-factor and integrin signals to promote cell migration. Nat Cell Biol, 2000. 2(5): p. 249-56.

69. Wennstrom, S. and J. Downward, Role of PI3 Kinase in activation of Ras and MAPK by EGF. Molecular \& Cellular Biology, 1999. 19: p. 4279-4288.

70. Fanger, G.R., N.L. Johnson, and G.L. Johnson, MEK kinases are regulated by EGF and selectively interact with Rac/Cdc42. Embo J, 1997. 16(16): p. 496172.

71. Marcoux, N. and K. Vuori, EGF receptor mediates adhesion-dependent activation of the Rac GTPase: a role for phosphatidylinositol 3-kinase and Vav2. Oncogene, 2003. 22(38): p. 6100-6.

72. Tamas, P., et al., Mechanism of epidermal growth factor regulation of Vav2, a guanine nucleotide exchange factor for Rac. J Biol Chem, 2003. 278(7): p. 5163-71.

73. Tu, S., et al., Epidermal growth factor-dependent regulation of Cdc42 is mediated by the Src tyrosine kinase. J Biol Chem, 2003. 278(49): p. 49293300. 
74. Gillgrass, A., et al., Epidermal growth factor receptor-dependent activation of Gab1 is involved in ErbB-2-mediated mammary tumor progression. Oncogene, 2003. 22(57): p. 9151-5.

75. De Luca, A., et al., The role of the EGFR signaling in tumor microenvironment. J Cell Physiol, 2008. 214(3): p. 559-67.

76. Normanno, N., et al., Epidermal growth factor receptor (EGFR) signaling in cancer. Gene, 2006. 366(1): p. 2-16.

77. Pryczynicz, A., et al., Expression of EGF and EGFR strongly correlates with metastasis of pancreatic ductal carcinoma. Anticancer Res, 2008. 28(2B): p. 1399-404.

78. Kim, H. and W.J. Muller, The role of the epidermal growth factor receptor family in mammary tumorigenesis and metastasis. Exp Cell Res, 1999. 253(1): p. 7887.

79. Pollard, J.W., Macrophages define the invasive microenvironment in breast cancer. J Leukoc Biol, 2008.

80. Xue, C., et al., Epidermal growth factor receptor overexpression results in increased tumor cell motility in vivo coordinately with enhanced intravasation and metastasis. Cancer Res, 2006. 66(1): p. 192-7.

81. Rameh, L.E. and L.C. Cantley, The role of phosphoinositide 3-kinase lipid products in cell function. J Biol Chem, 1999. 274(13): p. 8347-50. 
82. Sotsios, Y. and S.G. Ward, Phosphoinositide 3-kinase: a key biochemical signal for cell migration in response to chemokines. Immunol Rev, 2000. 177: p. 217-35.

83. Niggli, V., A membrane-permeant ester of phosphatidylinositol 3,4, 5trisphosphate (PIP(3)) is an activator of human neutrophil migration. FEBS Lett, 2000. 473(2): p. 217-21.

84. Weiner, O.D., et al., A PtdInsP(3)- and Rho GTPase-mediated positive feedback loop regulates neutrophil polarity. Nat Cell Biol, 2002. 4(7): p. 509-13.

85. Rossman, K.L., C.J. Der, and J. Sondek, GEF means go: turning on RHO GTPases with guanine nucleotide-exchange factors. Nat Rev Mol Cell Biol, 2005. 6(2): p. 167-80.

86. Barber, M.A. and H.C. Welch, PISK and RAC signalling in leukocyte and cancer cell migration. Bull Cancer, 2006. 93(5): p. E44-52.

87. Giglione, C. and A. Parmeggiani, Raf-1 is involved in the regulation of the interaction between guanine nucleotide exchange factor and Ha-ras. Evidences for a function of Raf-1 and phosphatidylinositol 3-kinase upstream to Ras. J Biol Chem, 1998. 273(52): p. 34737-44.

88. Hu, Q., et al., Ras-dependent induction of cellular responses by constitutively active phosphatidylinositol-3 kinase. Science, 1995. 268(5207): p. 100-2.

89. Rodriguez-Viciana, P., et al., Phosphatidylinositol-3-OH kinase as a direct target of Ras. Nature, 1994. 370(6490): p. 527-32. 
90. Rodriguez-Viciana, P., et al., Role of phosphoinositide 3-OH kinase in cell transformation and control of the actin cytoskeleton by Ras. Cell, 1997. 89(3): p. 457-67.

91. Kapeller, R. and L.C. Cantley, Phosphatidylinositol 3-kinase. Bioessays, 1994. 16(8): p. 565-76.

92. Lopez-llasaca, M., et al., Linkage of G protein-coupled receptors to the MAPK signaling pathway through PI 3-kinase gamma. Science, 1997. 275(5298): p. 394-7.

93. Chaudhary, A., et al., Phosphatidylinositol 3-kinase regulates Raf1 through Pak phosphorylation of serine 338. Curr Biol, 2000. 10(9): p. 551-4.

94. Sun, H., et al., Regulation of the protein kinase Raf-1 by oncogenic Ras through phosphatidylinositol 3-kinase, Cdc42/Rac and Pak. Curr Biol, 2000. 10(5): p. 281-4.

95. Takahashi, A. and W.C. Earnshaw, ICE-related proteases in apoptosis. Curr Opin Genet Dev, 1996. 6(1): p. 50-5.

96. Hale, A.J., et al., Apoptosis: Molecular Regulation of Cell Death. European Journal of Biochemistry, 1996. 236(1): p. 1-26.

97. Boatright, K.M., et al., $A$ unified model for apical caspase activation. Molecular Cell, 2003. 11(2): p. 529-41.

98. Chang, D.W., et al., Interdimer processing mechanism of procaspase-8 activation. Embo J, 2003. 22(16): p. 4132-42. 
99. Boatright, K.M., et al., Activation of caspases-8 and -10 by $F L I P(L)$. Biochem J, 2004. 382(Pt 2): p. 651-7.

100. Chang, D.W., et al., c-FLIP $(L)$ is a dual function regulator for caspase-8 activation and CD95-mediated apoptosis. EMBO Journal, 2002. 21(14): p. 3704-14.

101. Wagner, K.W., I.H. Engels, and Q.L. Deveraux, Caspase-2 Can Function Upstream of Bid Cleavage in the TRAIL Apoptosis Pathway. J. Biol. Chem., 2004. 279(33): p. 35047-35052.

102. Slee, E.A., C. Adrain, and S.J. Martin, Serial killers: ordering caspase activation events in apoptosis. Cell Death Differ, 1999. 6(11): p. 1067-74.

103. Stegh, A.H., et al., Identification of the Cytolinker Plectin as a Major Early In Vivo Substrate for Caspase 8 during CD95- and Tumor Necrosis Factor Receptor-Mediated Apoptosis. Mol. Cell. Biol., 2000. 20(15): p. 5665-5679.

104. Droin, N., et al., Involvement of caspase-2 long isoform in Fas-mediated cell death of human leukemic cells. Blood, 2001. 97(6): p. 1835-1844.

105. Lavrik, I.N., et al., Caspase-2 is activated at the CD95 death-inducing signaling complex in the course of CD95-induced apoptosis. Blood, 2006. 108(2): p. 55965.

106. Duan, H. and V.M. Dixit, RAIDD is a new 'death' adaptor molecule. Nature, 1997. 385(6611): p. 86-9. 
107. Ahmad, M., et al., CRADD, a novel human apoptotic adaptor molecule for caspase-2, and FasL/tumor necrosis factor receptor-interacting protein RIP. Cancer Res, 1997. 57(4): p. 615-9.

108. Tinel, A. and J. Tschopp, The PIDDosome, a Protein Complex Implicated in Activation of Caspase-2 in Response to Genotoxic Stress. Science, 2004. 304(5672): p. 843-846.

109. Tu, S., et al., In situ trapping of activated initiator caspases reveals a role for caspase-2 in heat shock-induced apoptosis. Nat Cell Biol, 2006. 8(1): p. 72.

110. Castedo, M., et al., Cell death by mitotic catastrophe: a molecular definition. Oncogene, 2004. 23(16): p. 2825-37.

111. Castedo, M., et al., Mitotic catastrophe constitutes a special case of apoptosis whose suppression entails aneuploidy. Oncogene, 2004. 23(25): p. 4362-70.

112. Baptiste-Okoh, N., A.M. Barsotti, and C. Prives, Caspase 2 is both required for p53-mediated apoptosis and downregulated by $p 53$ in a p21-dependent manner. Cell Cycle, 2008. 7(9).

113. Baptiste-Okoh, N., A.M. Barsotti, and C. Prives, A role for caspase 2 and PIDD in the process of p53-mediated apoptosis. Proc Natl Acad Sci U S A, 2008. 105(6): p. 1937-42.

114. Vakifahmetoglu, $\mathrm{H}_{\text {., }}$ et al., DNA damage induces two distinct modes of cell death in ovarian carcinomas. Cell Death Differ, 2008. 15(3): p. 555-66. 
115. Vakifahmetoglu, $\mathrm{H}$., et al., Functional connection between $p 53$ and caspase-2 is essential for apoptosis induced by DNA damage. Oncogene, 2006. 25(41): $\mathrm{p}$. 5683-92.

116. Cuenin, S., et al., p53-induced protein with a death domain (PIDD) isoforms differentially activate nuclear factor-kappaB and caspase-2 in response to genotoxic stress. Oncogene, 2008. 27(3): p. 387-96.

117. Janssens, S., et al., PIDD mediates NF-kappaB activation in response to DNA damage. Cell, 2005. 123(6): p. 1079-92.

118. Zhivotovsky, B. and S. Orrenius, Caspase-2 function in response to DNA damage. Biochem Biophys Res Commun, 2005. 331(3): p. 859-67.

119. Enoksson, M., et al., Caspase-2 permeabilizes the outer mitochondrial membrane and disrupts the binding of cytochrome $c$ to anionic phospholipids. J Biol Chem, 2004. 279(48): p. 49575-8.

120. Lassus, P., X. Opitz-Araya, and Y. Lazebnik, Requirement for caspase-2 in stress-induced apoptosis before mitochondrial permeabilization. Science, 2002. 297(5585): p. 1352-4.

121. Ho, L.H., et al., Caspase-2 is required for cell death induced by cytoskeletal disruption. Oncogene, 2008.

122. Troy, C.M. and M.L. Shelanski, Caspase-2 redux. Cell Death Differ, 2003. 10(1): p. 101-7.

123. Guo, Y., et al., Caspase-2 induces apoptosis by releasing proapoptotic proteins from mitochondria. Journal of Biological Chemistry, 2002. 277(16): p. 13430-7. 
124. Mancini, M., et al., Caspase-2 Is Localized at the Golgi Complex and Cleaves Golgin-160 during Apoptosis. J. Cell Biol., 2000. 149(3): p. 603-612.

125. Zheng, T.S., et al., Caspase knockouts: matters of life and death. Cell Death Differ, 1999. 6(11): p. 1043-53.

126. Varfolomeev, E.E., et al., Targeted disruption of the mouse Caspase 8 gene ablates cell death induction by the TNF receptors, Fas/Apo1, and DR3 and is lethal prenatally. Immunity, 1998. 9(2): p. 267-76.

127. Kang, T.B., et al., Caspase-8 serves both apoptotic and nonapoptotic roles. J Immunol, 2004. 173(5): p. 2976-84.

128. Hakem, R., et al., Differential requirement for caspase 9 in apoptotic pathways in vivo. Cell, 1998. 94(3): p. 339-52.

129. Kuida, K., et al., Reduced apoptosis and cytochrome c-mediated caspase activation in mice lacking caspase 9. Cell, 1998. 94(3): p. 325-37.

130. Bergeron, L., et al., Defects in regulation of apoptosis in caspase-2deficient mice. Genes Dev., 1998. 12(9): p. 1304-1314.

131. Kuida, K., et al., Decreased apoptosis in the brain and premature lethality in CPP32-deficient mice. Nature, 1996. 384(6607): p. 368-72.

132. Abraham, M.C. and S. Shaham, Death without caspases, caspases without death. Trends Cell Biol, 2004. 14(4): p. 184-93. 
133. Schwerk, C. and K. Schulze-Osthoff, Non-apoptotic functions of caspases in cellular proliferation and differentiation. Biochem Pharmacol, 2003. 66(8): p. 1453-8.

134. Ishizaki, Y., M.D. Jacobson, and M.C. Raff, A role for caspases in lens fiber differentiation. J Cell Biol, 1998. 140(1): p. 153-8.

135. Carlile, G.W., D.H. Smith, and M. Wiedmann, Caspase-3 has a nonapoptotic function in erythroid maturation. Blood, 2004. 103(11): p. 4310-6.

136. Zermati, Y., et al., Caspase activation is required for terminal erythroid differentiation. J Exp Med, 2001. 193(2): p. 247-54.

137. Sordet, O., et al., Specific involvement of caspases in the differentiation of monocytes into macrophages. Blood, 2002. 100(13): p. 4446-53.

138. Ahmad, M., et al., Identification and characterization of murine caspase-14, a new member of the caspase family. Cancer Res, 1998. 58(22): p. 5201-5.

139. Van de Craen, M., et al., Identification of a new caspase homologue: caspase14. Cell Death Differ, 1998. 5(10): p. 838-46.

140. Alibardi, L., E. Tschachler, and L. Eckhart, Distribution of caspase-14 in epidermis and hair follicles is evolutionarily conserved among mammals. Anat Rec A Discov Mol Cell Evol Biol, 2005. 286(2): p. 962-73.

141. Eckhart, L., et al., Terminal differentiation of human keratinocytes and stratum corneum formation is associated with caspase-14 activation. J Invest Dermatol, 2000. 115(6): p. 1148-51. 
142. Fischer, H., et al., Stratum corneum-derived caspase-14 is catalytically active. FEBS Lett, 2004. 577(3): p. 446-50.

143. Barnoy, S. and N.S. Kosower, Caspase-1-induced calpastatin degradation in myoblast differentiation and fusion: cross-talk between the caspase and calpain systems. FEBS Lett, 2003. 546(2-3): p. 213-7.

144. Fernando, P., et al., Caspase 3 activity is required for skeletal muscle differentiation. Proc Natl Acad Sci U S A, 2002. 99(17): p. 11025-30.

145. Graves, J.D., et al., Caspase-mediated activation and induction of apoptosis by the mammalian Ste20-like kinase Mst1. Embo J, 1998. 17(8): p. 2224-34.

146. Vaisid, T., N.S. Kosower, and S. Barnoy, Caspase-1 activity is required for neuronal differentiation of PC12 cells: cross-talk between the caspase and calpain systems. Biochim Biophys Acta, 2005. 1743(3): p. 223-30.

147. Jeon, B.S., et al., Activation of caspase-3 in developmental models of programmed cell death in neurons of the substantia nigra. J Neurochem, 1999. 73(1): p. 322-33.

148. Kermer, P., et al., Caspase-9: involvement in secondary death of axotomized rat retinal ganglion cells in vivo. Brain Res Mol Brain Res, 2000. 85(1-2): $p$. 144-50.

149. Kermer, P., et al., Inhibition of CPP32-like proteases rescues axotomized retinal ganglion cells from secondary cell death in vivo. J Neurosci, 1998. 18(12): p. 4656-62. 
150. Kermer, P., et al., Activation of caspase-3 in axotomized rat retinal ganglion cells in vivo. FEBS Lett, 1999. 453(3): p. 361-4.

151. Yan, X.X., et al., Expression of active caspase-3 in mitotic and postmitotic cells of the rat forebrain. J Comp Neurol, 2001. 433(1): p. 4-22.

152. Urase, K., et al., Detection of activated caspase-3 (CPP32) in the vertebrate nervous system during development by a cleavage site-directed antiserum. Brain Res Dev Brain Res, 1998. 111(1): p. 77-87.

153. Gdynia, G., et al., Basal caspase activity promotes migration and invasiveness in glioblastoma cells. Mol Cancer Res, 2007. 5(12): p. 1232-40.

154. De Botton, S., et al., Platelet formation is the consequence of caspase activation within megakaryocytes. Blood, 2002. 100(4): p. 1310-7.

155. Sapet, C., et al., Thrombin-induced endothelial microparticle generation: identification of a novel pathway involving ROCK-Il activation by caspase-2. Blood, 2006. 108(6): p. 1868-1876.

156. Jy, W., et al., Endothelial microparticles induce formation of platelet aggregates via a von Willebrand factor/ristocetin dependent pathway, rendering them resistant to dissociation. J Thromb Haemost, 2005. 3(6): p. 1301-8.

157. Geisbrecht, E.R. and D.J. Montell, A role for Drosophila IAP1-mediated caspase inhibition in Rac-dependent cell migration. Cell, 2004. 118(1): p. 11125.

158. Oshima, K., et al., IKK epsilon regulates $F$ actin assembly and interacts with Drosophila IAP1 in cellular morphogenesis. Curr Biol, 2006. 16(15): p. 1531-7. 
159. Kuranaga, E., et al., Drosophila IKK-related kinase regulates nonapoptotic function of caspases via degradation of IAPs. Cell, 2006. 126(3): p. 583-96.

160. Montell, D.J., A kinase gets caspases into shape. Cell, 2006. 126(3): p. 450-2.

161. Huh, J.R., M. Guo, and B.A. Hay, Compensatory proliferation induced by cell death in the Drosophila wing disc requires activity of the apical cell death caspase Dronc in a nonapoptotic role. Curr Biol, 2004. 14(14): p. 1262-6.

162. Arama, E., J. Agapite, and H. Steller, Caspase activity and a specific cytochrome $C$ are required for sperm differentiation in Drosophila. Dev Cell, 2003. 4(5): p. 687-97.

163. Muro, I., et al., The Drosophila caspase Ice is important for many apoptotic cell deaths and for spermatid individualization, a nonapoptotic process. Development, 2006. 133(17): p. 3305-15.

164. Huh, J.R., et al., Multiple apoptotic caspase cascades are required in nonapoptotic roles for Drosophila spermatid individualization. PLoS Biol, 2004. 2(1): p. E15.

165. Barnhart, B.C., et al., CD95 ligand induces motility and invasiveness of apoptosis-resistant tumor cells. Embo J, 2004. 23(15): p. 3175-85.

166. Hill, J.M., et al., Recognition of ERK MAP kinase by PEA-15 reveals a common docking site within the death domain and death effector domain. Embo J, 2002. 21(23): p. 6494-504. 
167. Luschen, S., et al., The Fas-associated death domain protein/caspase-8/c-FLIP signaling pathway is involved in TNF-induced activation of ERK. Exp Cell Res, 2005. 310(1): p. 33-42.

168. Condorelli, G., et al., PED/PEA-15: an anti-apoptotic molecule that regulates FAS/TNFR1-induced apoptosis. Oncogene, 1999. 18(31): p. 4409-15.

169. Estelles, A., C.A. Charlton, and H.M. Blau, The phosphoprotein protein PEA-15 inhibits Fas- but increases TNF-R1-mediated caspase-8 activity and apoptosis. Dev Biol, 1999. 216(1): p. 16-28.

170. Formstecher, E., et al., PEA-15 mediates cytoplasmic sequestration of ERK MAP kinase. Dev Cell, 2001. 1(2): p. 239-50.

171. Ramos, J.W., et al., Death effector domain protein PEA-15 potentiates Ras activation of extracellular signal receptor-activated kinase by an adhesionindependent mechanism. Mol Biol Cell, 2000. 11(9): p. 2863-72.

172. Hsu, H., et al., TNF-dependent recruitment of the protein kinase RIP to the TNF receptor-1 signaling complex. Immunity, 1996. 4(4): p. 387-96.

173. Shikama, Y., M. Yamada, and T. Miyashita, Caspase-8 and caspase-10 activate NF-kappaB through RIP, NIK and IKKalpha kinases. Eur J Immunol, 2003. 33(7): p. 1998-2006.

174. Zhang, J., et al., Fas-mediated apoptosis and activation-induced T-cell proliferation are defective in mice lacking FADD/Mort1. Nature, 1998. 392(6673): p. 296-300. 
175. Beisner, D.R., et al., The requirements for Fas-associated death domain signaling in mature $T$ cell activation and survival. J Immunol, 2003. 171(1): p. 247-56.

176. Zhang, J., et al., FADD-deficient $T$ cells exhibit a disaccord in regulation of the cell cycle machinery. J Biol Chem, 2001. 276(32): p. 29815-8.

177. Algeciras-Schimnich, A., B.C. Barnhart, and M.E. Peter, Apoptosisindependent functions of killer caspases. Curr Opin Cell Biol, 2002. 14(6): p. 721-6.

178. Newton, K., et al., A dominant interfering mutant of FADD/MORT1 enhances deletion of autoreactive thymocytes and inhibits proliferation of mature $T$ lymphocytes. Embo J, 1998. 17(3): p. 706-18.

179. Sehra, S. and A.L. Dent, Caspase function and the immune system. Crit Rev Immunol, 2006. 26(2): p. 133-48.

180. Yeh, W.C., et al., FADD: essential for embryo development and signaling from some, but not all, inducers of apoptosis. Science, 1998. 279(5358): p. 1954-8.

181. Walsh, C.M., et al., A role for FADD in $T$ cell activation and development. Immunity, 1998. 8(4): p. 439-49.

182. Su, H., et al., Requirement for caspase-8 in NF-kappaB activation by antigen receptor. Science, 2005. 307(5714): p. 1465-8.

183. Zhang, N. and Y.W. He, An essential role for C-FLIP in the efficient development of mature T lymphocytes. J Exp Med, 2005. 202(3): p. 395-404. 
184. Lens, S.M., et al., The caspase 8 inhibitor c-FLIP $(L)$ modulates $T$-cell receptorinduced proliferation but not activation-induced cell death of lymphocytes. Mol Cell Biol, 2002. 22(15): p. 5419-33.

185. Fang, L.W., et al., Phosphatidylinositide 3-kinase priming couples C-FLIP to $T$ cell activation. J Biol Chem, 2004. 279(1): p. 13-8.

186. Kataoka, T., et al., The caspase-8 inhibitor FLIP promotes activation of NFkappaB and Erk signaling pathways. Curr Biol, 2000. 10(11): p. 640-8.

187. Li, J., et al., Caspase-11 regulates cell migration by promoting Aip1-Cofilinmediated actin depolymerization. Nat Cell Biol, 2007. 9(3): p. 276.

188. Kong, J.Y. and S.W. Rabkin, The association between RhoB and caspase-2: changes with lovastatin-induced apoptosis. Biochem Cell Biol, 2005. 83(5): p. 608-19.

189. Wang, J. and M.J. Lenardo, Roles of caspases in apoptosis, development, and cytokine maturation revealed by homozygous gene deficiencies. J Cell Sci, 2000. 113 (Pt 5): p. 753-7.

190. Yang, L., et al., Coexistence of high levels of apoptotic signaling and inhibitor of apoptosis proteins in human tumor cells: implication for cancer specific therapy. Cancer Res, 2003. 63(20): p. 6815-24.

191. Yoo, N.J., et al., Loss of caspase-2, -6 and -7 expression in gastric cancers. Apmis, 2004. 112(6): p. 330-5. 
192. Teitz, T., et al., Caspase 8 is deleted or silenced preferentially in childhood neuroblastomas with amplification of MYCN.[comment]. Nature Medicine, 2000. 6(5): p. 529-35. 


\title{
Chapter 2
}

\section{Caspase-8 promotes cellular migration and adhesion}

\author{
Portions of this Chapter were published in:
}

Helfer, B, B Boswell, D Finlay, A Cipres, K Vuori, T B Kang, D Wallach, A Dorfleutner, J Lahti, D Flynn, S Frisch. Caspase-8 promotes cell motility and calpain activity under nonapoptotic conditions. Cancer Research 2006; 66: 4273-4278 


\section{Abstract}

Significant caspase-8 activity has been found in normal and certain tumor cells, suggesting that caspase-8 possesses an alternative, nonapoptotic function that may contribute to tumor progression. In this article, we report that caspase-8 promotes cell motility. In particular, caspase- 8 is required for the optimal activation of calpains, Rac and lamellipodial assembly. This represents a novel nonapoptotic function of caspase8 acting at the intersection of the caspase and calpain proteolytic pathways to coordinate cell death vs. cell motility signaling. 


\section{Introduction}

Caspases are typically known for their role in preventing cells with abnormalities from growing and proliferating. Caspase-8 expression and activation, however, is altered in both normal and cancer cancer cells in certain situations. Of note, breast and pancreatic cancers and glioblastomas exhibit significant caspase-8 activity [1-3], which could hold unique non-apoptotic properties. This reveals a potential nonapoptotic role of caspase-8 in tumor progression and normal cell biology.

As reviewed in Chapter 1, non-apoptotic roles are emerging for several caspases. Prior to this study, DRONC, the Drosophila caspase homolog of caspases 2 and 9, was implicated in boarder cell migration via a mechanism involving an interaction of DIAP with the Rac pathway [4]. Additionally, CD95 signaling has been implicated in the promotion of cellular invasion [5]. Under FasL stimulation, cells resistant to caspase-8 induced apoptosis exhibited increased cellular invasion in a NF-kappaB, Erk, and caspase-8 mediated mechanism. Since the publication of this study, additional caspases have been implicated in the promotion of cellular migration $[6,7]$.

The phenotype of caspase- 8 knockout mice and tissue specific caspase-8 knockouts demonstrates embryonic lethality due to the inability to assemble a functional circulatory system [8,9] and proper neural tube closure [8], as discussed in the Introduction. The failure of neural tube and circulatory system closure cannot be explained by a lack of apoptosis, thus it would not be surprising for caspase-8 to

possess a non-apoptotic role involving cellular migration. In this study we 
demonstrate that caspase- 8 regulates cell motility. Furthermore, calpains, which are deeply rooted in the promotion of migration (reviewed in [10]), are implicated in caspase-8 promoted migration. Consequently, we also demonstrate that caspase-8 also regulates cellular processes dependent upon calpain activity, such as Rac activation, assembly of lamellipodia, and the proper execution of cytokinesis. This reveals a potential nonapoptotic role of caspase- 8 in both tumor progression and normal cellular biology. 


\section{Materials and Methods}

Cell lines. Mouse embryo fibroblasts derived from caspase-8-null, caspase-3-null or isogenic wild-type control mice were characterized previously $[9,11]$. WTNR6 cells [12] were provided by Alan Wells (University of Pittsburgh); to generate derivatives expressing the cowpox virus-derived caspase-8 inhibitory protein crmA [13], the HAtagged crmA gene was retrovirally transduced using the vector MSCV-IRES-puro in the gp2+293 cell packaging system (Invitrogen). NB7 cells that were infected with empty GFP retrovirus or rescued with human caspase-8 retrovirus [14] were provided by J. Lahti (St. Jude's Research Institute). MDA-MB231 (American Type Culture Collection) were transduced with crmA as described above. Bcl 460 cells were kindly provided by Dr Rojanasakul (West Virginia University [15]). Cells were maintained in Dulbecco's Modified Eagle's Medium (MEFs, MDA-MB231), Advanced MEM (WTNR6), RPMI1640 (NB7), supplemented with 10\% fetal bovine serum and 1X penicillin-streptomycin-glutamine (Invitrogen).

Cell lines with inducible caspase-8 expression were generated using the Rheoswitch system (www.neb.com). Caspase- $8^{-/-}$MEFs (W102) were co-electroporated with a 4:1 ratio of the "Rheoswitch" activator/repressor-expressing plasmid -NEBR-R1: pcDNA3.1hyg and colonies were selected in $500 \mu \mathrm{g} / \mathrm{ml}$ hygromycin. Colonies were expanded and screened for expression of the VP16 moiety of the hybrid activator protein by Western blotting and for induction of luciferase activity after transient transfection of luciferase/pNEBR-X1. Wild-type mouse caspase-8 cDNAs was subcloned into the pNEBR-X1 target plasmid and co-electroporated in a 4:1 ratio with 
pBABE-puro. Colonies were selected in $5 \mu \mathrm{g} / \mathrm{ml}$ puromycin and screened for caspase- 8 expression by Western blotting with the anti-mouse-caspase- 8 antibody $1 \mathrm{G} 12$ (Alexis) after induction with the compound RSL1. A Western blot of the induction time course is shown below (figure 1). The level of caspase-8 expression at 24 hours of induction was approximately $30-50 \%$ of the level in wild-type MEFs (data not shown).

Cell motility assays. Method 1. Electrical Cell-Substratum Impedance Sensing (ECIS). Cells were plated on the chambers $\left(\sim 1 \mathrm{~cm}^{2}\right)$ of a single-electrode ECIS arrays (Applied Biophysics, Troy, New York; www.biophysics.com) that were pre-coated with fibronectin. After growing the cells to confluence, equal coverage of the electrodes was checked by measuring the initial impedance value (average $=1924$ ohms for caspase-8-/- cells, 2598 for caspase-8+/+ cells). The cells attached to the small electrode disc $(0.25 \mathrm{~mm}$ diameter) were selectively ablated by applying a $4 \mathrm{~V}$ pulse for 10 seconds, causing the impedance drop seen at zero hours. The impedance increase due to the surrounding cells migrating back onto the electrode was then monitored at $40 \mathrm{kHz}$ frequency over the indicated time course; migration was in the presence of $10 \%$ serum and was carried out in triplicate chambers with one additional "unwounded" chamber serving as a negative control. Data were exported to Excel for calculation of slopes.

Method 2. Conventional wound-healing. Cells were grown to confluence on fibronectin-coated coverslips or $35 \mathrm{~mm}$ Mattek dishes, serum-starved overnight $(0.5 \%$ 
serum) and the monolayer was wounded with a pipet tip; EGF (10 nM) was then added. The wound was photographed at zero time and at the indicated time points using vertical and horizontal alignment marks made on the plastic well with a syringe needle. Three wound areas were traced and measured using the Axiovision software, averaged and represented here as the percent wound closure. Alternatively, one hour after attachment, a wound was generated, unattached cells were washed off and videomicroscopy was performed on the dish which was incubated on a heated stage in CO2-independent medium:CCM1 medium (1:1). In experiments using the rheoswitch inducible cell lines, cells were induced for 24 hours in the presence of 500 nM RSL1 prior to wounding.

Method 3. Single cell motility assays. Cells were plated onto fibronectin at low density for one hour in the absence of serum, re-stimulated with serum and recorded by videomicroscopy for 10 hours. The paths traveled by seven individual cells were traced and the total distance traveled by each cell was computed using the Axiovision software (interactive measurement module).

Method 4. Transwell/chemotaxis assays. Cell migration assays were performed with modified Boyden chambers, $6.5 \mathrm{~mm}$ diameter, $8 \mathrm{~mm}$ pore size (Transwell from Costar Corp.) according to the manufacturer's protocol. Briefly, after serum starvation of the MEF cells they were harvested with trypsin and quenched with Soybean trypsin inhibitor (Calbiochem). The lower compartment of the migration chamber was filled with 3T3-fibroblast conditioned media and $6 \times 10^{4}$ cells were suspended in 
DMEM/10mM Hepes/0.5\% BSA $1 \%$ penicillin-streptomycin and added to the upper compartment of the migration chamber. Migration chambers were incubated for $4 \mathrm{~h}$ at $37^{\circ} \mathrm{C}$ in $5 \% \mathrm{CO}_{2}$. After removal of stationary cells from the upper side of the membrane, migrated cells were fixed in 3.7\% paraformaldehyde in PBS and stained with $1 \%$ crystal violet in $2 \%$ ethanol. Membranes were extensively washed, the dye eluted with $10 \%$ acetic acid and absorbance was measured at $600 \mathrm{~nm}$. Results are expressed as $\%$ migrated cells relative to a control in which total adherent cell absorbance was measured.

Method 5. Fas Ligand stimulated motility. Motility assays were performed as previously mentioned for standard scratch assays or transwell assays with the following exceptions: FASL was incubated with FLAG antibody (Sigma) for 15 minutes and applied to cells at a concentration of $100 \mathrm{ng} / \mathrm{mL}$ FASL to stimulate motility in the presence of $2 \%$ fetal bovine serum (Invitrogen).

Rac activity assays. Cells were incubated overnight in DME medium with $0.5 \%$ fetal bovine serum, and detached by trypsinization and neutralized with an equal volume of $2.5 \mathrm{mg} / \mathrm{ml}$ soybean trypsin inhibitor or detached by treatment with Tryple Express (Invitrogen) and neutralized by ten-fold dilution in serum-free medium, followed by centrifugation. The cells were then resuspended in serum-free DME containing 20 $\mathrm{mg} / \mathrm{ml} \mathrm{BSA}$ and plated onto a series of $60 \mathrm{~mm}$ dishes that had been pre-coated with $20 \mu \mathrm{g} / \mathrm{ml}$ fibronectin (Calbiochem) and blocked with 3\% BSA. At the indicated times, cells were scraped into $0.4 \mathrm{ml}$ of cold lysis buffer $(0.5 \%$ TritonX100/50 mM Tris $\mathrm{pH}$ 
7.5/150 mM NaCl/10\% glycerol/10 mM MgCl $2+1 X$ complete Roche protease inhibitors, EDTA-free) and pre-cleared. Ten $\mu \mathrm{g}$ of bacterially expressed GST-PAK (CRIB domain) protein were added and the samples incubated on ice for $1 \mathrm{hr}$, followed by addition of $30 \mu \mathrm{L}$ of a $50 \%$ slurry of glutathione-sepharose (Pharmacia; equilibrated in lysis buffer containing $20 \mathrm{mg} / \mathrm{ml} \mathrm{BSA}$ ) and further incubation for one hour in the coldroom on a rotation device. Beads were washed three times in lysis buffer and analyzed on Western blots for rac using monoclonal antibodies from BDPharmingen, peroxidase-labeled anti-mouse antibody (Invitrogen) and Pierce West Pico chemiluminescence kit.

Immunofluorescence. Cell grown on coverslips were fixed with cold methanol for the tubulin/pericentrin staining or with $4 \%$ paraformaldehyde for the cortactin/actin staining, followed by five-minute permeabilization with $0.1 \%$ TritonX100 for the latter. Cells were stained with anti-a-tubulin mouse monoclonal antibody (DM1A) (Calbiochem), anti-pericentrin pAb (Covance), anti-cortactin mAb (S. Weed) or with GM130, Golgi matrix protein of $130 \mathrm{kDA}$, antibody (BD Transduction Laboratories) followed by Alexa-488 or Alexa-594 labeled secondary antibodies (Pierce) and mounting with Vectashield containing DAPI (Vector Labs). Images were collected on a Zeiss Axiovert 200M microscope using AxioVision software and imported into Photoshop, where individual color intensities and contrast were adjusted for clarity of presentation; image intensities are subjectively accurate but non-quantitative. 
Cellular Adhesion Assay. Electrical Cell-Substratum Impedance Sensing (ECIS). Cells were counted $\left(5 \times 10^{\wedge} 5\right.$ cells/well) and let attach to the chambers $\left(\sim 1 \mathrm{~cm}^{2}\right)$ of a ten-electrode ECIS array (Applied Biophysics, Troy, New York; www.biophysics.com) that were pre-coated with fibronectin. Adhesion was done in the absence of serum and was observed until a steady impedance value was reached. As the cells attached to the electrodes of the ECIS array, the impedance increased. The impedance was monitored at $40 \mathrm{kHz}$ frequency and was carried out in triplicate chamber. Data were exported to Excel for calculation of slopes.

Cellular Invasion Assay. Invasion assays were done according to the BD Biocoat Matrigel Invasion Chamber guidelines with few exceptions. Briefly, invasion chabers were allowed to warm to room temperature before rehydrating matrigel with $1 \mathrm{x}$ phosphate buffered saline (PBS) for 2 hours. $5 \times 10^{4}$ cells $/ \mathrm{mL}$ were counted. $0.5 \mathrm{~mL}$ $\left(2.5 \times 10^{4}\right.$ cells $)$ were added to the top chamber of a 24 well plate insert $+/-100 \mathrm{ng} / \mathrm{mL}$ FASL in $0.5 \%$ serum containing media and allowed to invade for 6-72 hours toward serum and FASL containing media. 


\section{Results}

Caspase-8 regulates cell motility. We assayed mouse embryo fibroblasts (MEFs) obtained from caspase-8-knockout mice or wild type MEFs [9] for motility utilizing an Electrical Cell-Substratum Impedance Sensing (ECIS) instrument [16]. Caspase-8 deficient cells exhibited decreased motility (figure 2A). These results were mirrored in conventional wound-healing assays (figure 2B). The viral caspase-1/8 inhibitor protein crmA [13] was also expressed in the wild-type MEFs, MDA-MB231 mammary carcinoma cells, and WTNR6 fibroblasts. CrmA expression inhibited motility via transwell chemotaxis and via standard wound healing assay analysis (figure $2 \mathrm{C}$ and 2D). Caspase-8 expression was also re-established in an inducible manner in caspase-8 knockout MEFs. Similarly to wild type MEFS, induction of caspase-8 restored cellular migration to knockout MEFs (figure 2E). The addition of crmA, and analysis of inducible caspase-8 re-expression confirm that a decrease in cellular migration was not an irreversible defect of the caspase-8 knockout cell lines. Furthermore, caspase-3 did not stimulate cell motility (figure 2F). This result suggests that caspase-8 may act directly to promote cell motility, rather than through its downstream effector caspase-3. Additional downstream caspases cannot be excluded.

Because mammary carcinomas exhibited decreased transwell migration upon crmA expression, other tumor lines were examined for caspase-8 promoted cell migration. Similar results were obtained in NB7 neuroblastoma cells [14] reconstituted with caspase-8 and in MDA-MB-231 mammary carcinoma cells made to express crmA 
(figure 2G, H, I). Caspase-8's promotion of cellular migration is thus not limited to normal cell biology; it is also an important factor in cancer cell biology and tumor progression.

Activation of caspase-8 by FASL promotes invasion (through NF-kB, Erk, and caspase 8 [5]). Consistent with the results of Barnhart et al. [5] we found that FASL stimulated cellular invasion and that caspase-8 was important for this process (Figure 3 A,B,C). These results suggest that caspase- 8 expression in tumors resistant to caspase-8 induced apoptosis may actually promote tumor metastasis through increased cellular migration and invasion.

Caspase-8 affects cellular processes downstream of calpain activation. Work from our laboratory demonstrated that caspase- 8 is important for the activation of calpain activity by EGF [17]. To confirm that caspase-8 regulates the calpain pathway, we examined several calpain-dependent processes for caspase-8dependence: Rac activation, lamellipodial assembly, cell adhesion and proper cellular division [10, 18-22]. Caspase- $8^{-/-}$cells exhibited a. decreased levels of Rac activation when compared to control cells (figure 4); b. fewer lamellipodia than their caspase-8 expressing counterparts (figure 5A,B); c. decreased cell adhesion, measured by ECIS (figure 5C); d. more multinucleate cells than their caspase-8 expressing counterparts (figure 5D). Calpains are also associated with cell polarity, however this point is only recently found in the literature and is yet to be well established [23]. Interestingly, capase-8 did not influence cell polarity (figure $5 \mathrm{E}$ ). 
Caspase-8 promotes Erk activation. We hypothesized that the connection of caspase 8 and calpain in the promotion of migration occurs through the coordination of the EGFR $\rightarrow$ MAPK/Erk signaling pathway. Both calpains and caspase- 8 have been liked to signaling pathways containing Erk [5, 12, 24-27]. Erk itself is associated with the phosphorylation of calpain and its activation [12] upon stimulation from EGFR. Consistent with this, EGF stimulation of cells lacking caspase-8 demonstrated attenuated activation (Figure 6). 


\section{Discussion and Future Studies}

Caspase-8, an initiator caspase in the process of apoptosis has been suggested to have roles in normal cell biology based upon its requirement for proper development of mice and other developmental requirements in tissue specific knockout animals [8, 9]. In this study we have shown that Caspase-8 is an important element in the promotion of cell migration. Moreover, caspase-8 is also required for enhanced cellular adhesion, invasion, lamellipodia projection, and proper cytokinesis. A comparison of caspase-8 expressing MEF against knockout MEF demonstrated an increased Rac activation upon cellular adhesion in cells expressing caspase-8 (Figure 4).

Additional data from our laboratory has shown that caspase-8 also contributes to calpain activation [17]. This data paired with caspase-8's ability to promote Erk activation makes Erk activation pathways an interesting target for caspase-8's promotion of cell migration. As stated in the Introduction (Chapter 1) calpain is an important mediator of cell migration (lamellipodia projection, cellular adhesion dynamics etc) and that Erk activation promotes calpain activity.

Caspase-8 has previously been linked to Erk activation in the context of CD95/FADD/caspase-8/c-FLIP, as reviewed in the introduction. CD95 (Fas/Apo-1) is a death receptor responsible for the formation of the death inducing signaling complex (DISC) [28] to promote apoptosis. Directly related to this study, CD95 signaling is nonapoptotically associated with invasion and motility through NF-kappaB, Erk and 
caspase-8 signaling [5] upon stimulation by FasL. In our model, motility and invasion are also influenced by caspase- 8 when stimulated/attracted by EGF, a response that is also mirrored with FasL stimulation.

While initially believed to be contradictory to our work, loss of caspase- 8 has been shown to increase metastasis in neuroblastoma cells [29]. Neuroblastoma cells often eliminate caspase-8 expression by hypermethylating the caspase-8 promoter [14], making it an ideal system in which to study the effects of caspase-8. Re-expression of caspase-8 is shown to restore motility in neuroblastoma cells (Figure 2H). Additionally, caspase-8 promotes cellular invasion (Figure $3[5]$ ). While our results would be believed to aid in metastasis, not inhibit it, the results of Stupack et al examine caspase-8 in an apoptotic context. Re-expression of caspase-8 can, paradoxically, suppress metastasis by conferring anoikis-sensitivity $[29,30]$. However, most metastatic tumor cells express caspase-8 but are, by definition, resistant to anoikis. Thus, in most human tumors, the presence or absence of caspase-8 is not the major determinant of anoikis-sensitivity.

Relevant to this study, it is important to note that the use of viral inhibitor crmA [13] should not be interpreted as solely blocking the catalytic activity of caspase-8. CrmA also binds to full length caspase- 8 and thus able to block the catalytic activity of full length caspase-8 as well as block potential protein;protein interactions [31]. Should the catalytic activity of caspase- 8 be required for the promotion of calpain activity, as we predict it is, crmA would be able to block this catalytic activity. Using Rheoswitch 
inducible MEF cells, we attempted to determine if the catalytic activity of caspase-8 was required for the promotion of cell migration and caplain activity, however, these results were inconclusive.

As stated, we hypothesize that the connection of caspase 8 and calpain in the promotion of migration occurs through the coordination of the EGFR $\rightarrow$ MAPK/Erk signaling pathway. In this model, Erk can be activated through the well established growth factor receptor $\rightarrow$ Ras $\rightarrow$ Raf $\rightarrow$ Mek pathway (reviewed [32, 33]) or through interaction of EGFR with FAK signaling to MEKK1 [34]. MEKK1 then activates Erk (Chapter 1, Figure 1) leading to the subsequent calpain activation. Additionally, calpain could be activated in a MEKK1 cleavage dependent manner. Supporting this hypothesis, caspase-8 and -3 can activate MEKK1 by direct cleavage ([35] and [27, 36] respectively). The dependence of caspase-8s proteolytic activity in these processes has yet to be addressed. Our current model predicts that the catalytic activity of caspase-8 is needed for calpain activity through the MEKK1 pathway. Potential pathways are overviewed in Figure 7. Currently, we are in the process of generating MEKK1 inducible Rheoswitch cells that express either wild type MEKK1 or the caspase-8 uncleavable mutant [35] of MEKK1 in MEKK1 knockout MEF [37]. These cells will initially be examined for their ability to promote cell migration, calpain activation/phosphorylation, and Erk activation. While we have yet to make a direct connection in our model between caspase-8 with MEKK1 signaling to calpain, this remains a focus of our laboratory. 
Figure 1

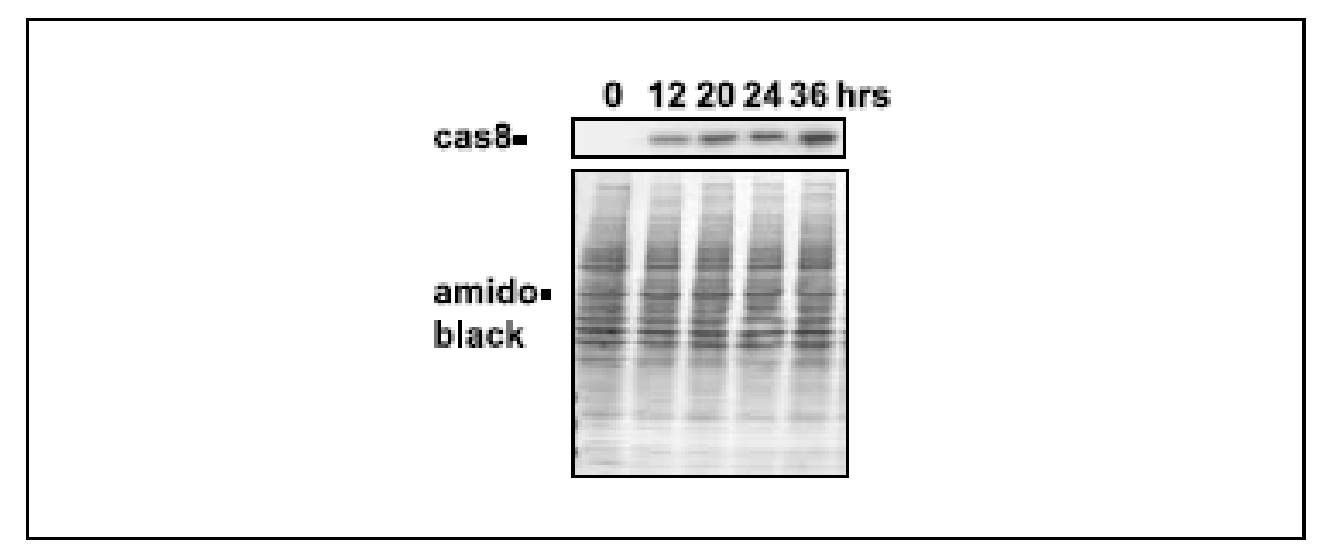

Figure 1. Inducible expression of caspase-8. 


\section{Figure 2}
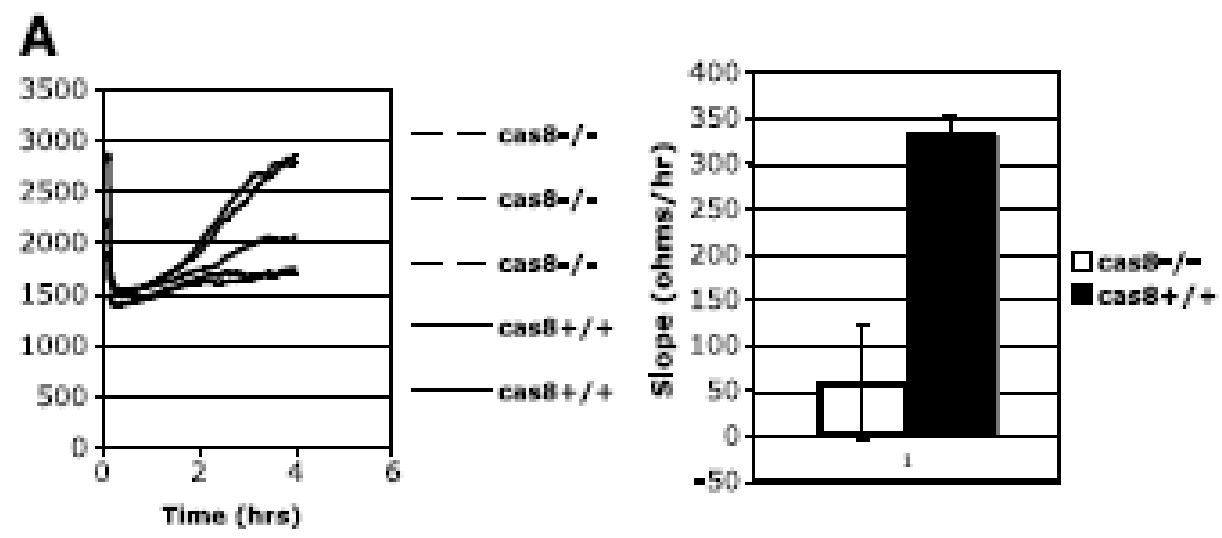

B

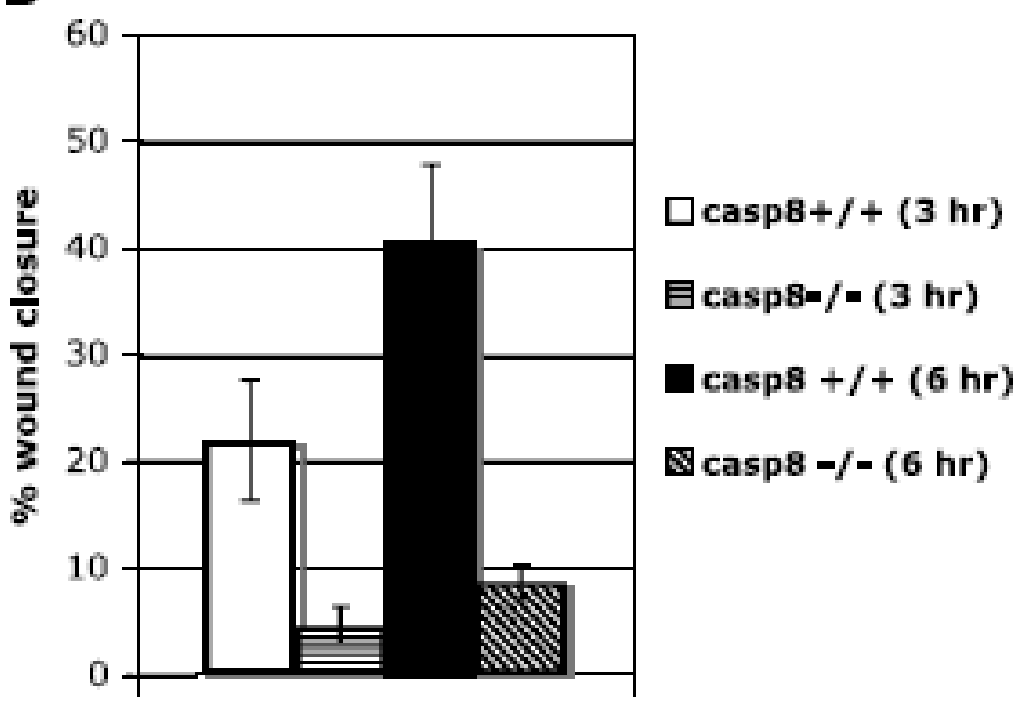

Figure 2. Caspase-8 promotes cell motility. (A). ECIS analysis of caspase- $8^{-1-}$ vs. caspase$8^{+/+}$mouse embryo fibroblasts (MEFs). (left-hand panel): Impedance vs. time plot. (right-hand panel): Graphic representation of slopes calculated from the linear part of the graphs in the ECIS tracing (after subtraction of slopes measured in "unwounded" wells). (B). Conventional wound healing assay, comparing caspase $-8^{-/-}$vs. caspase $-8^{+/+}$MEFs. 
C Transwell Migration 18hrs

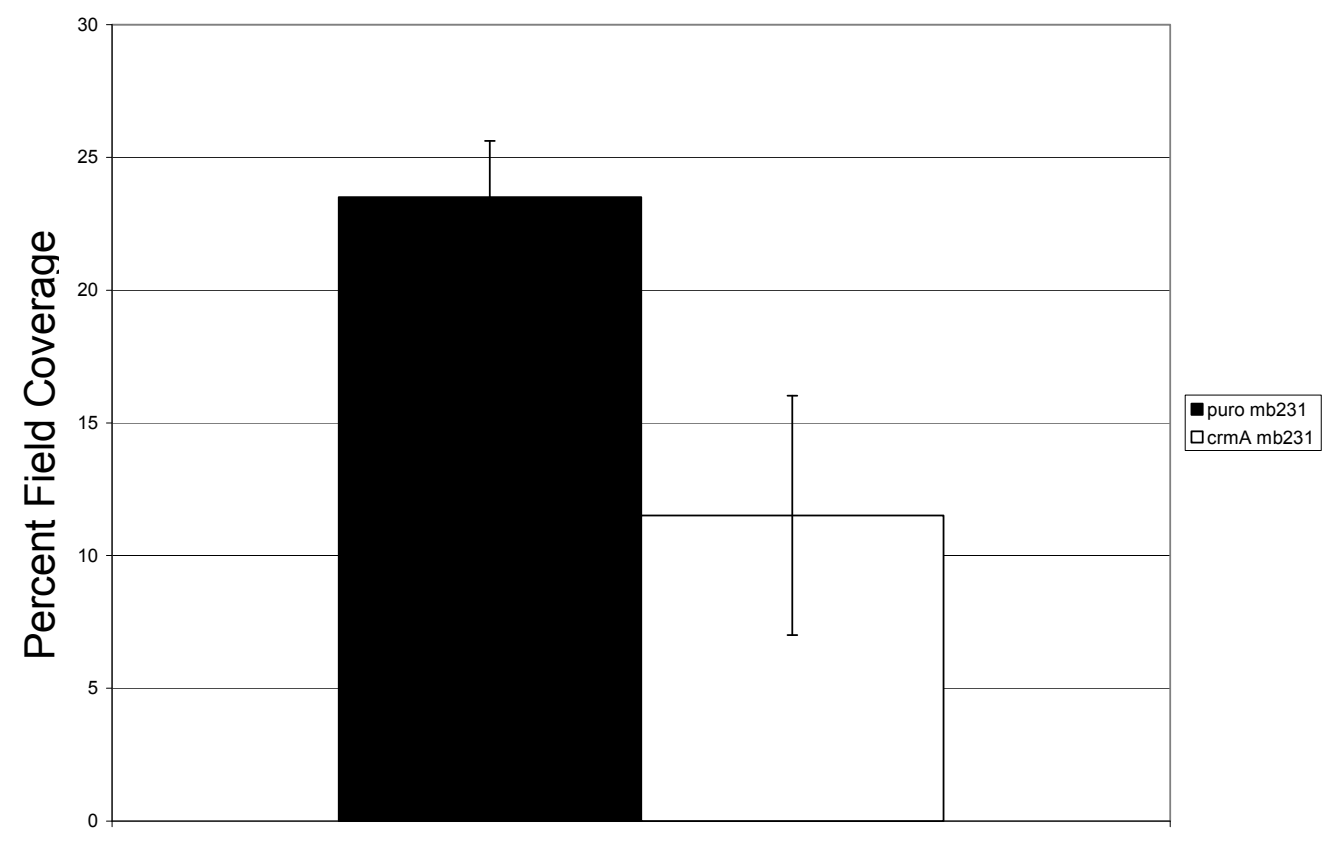

D
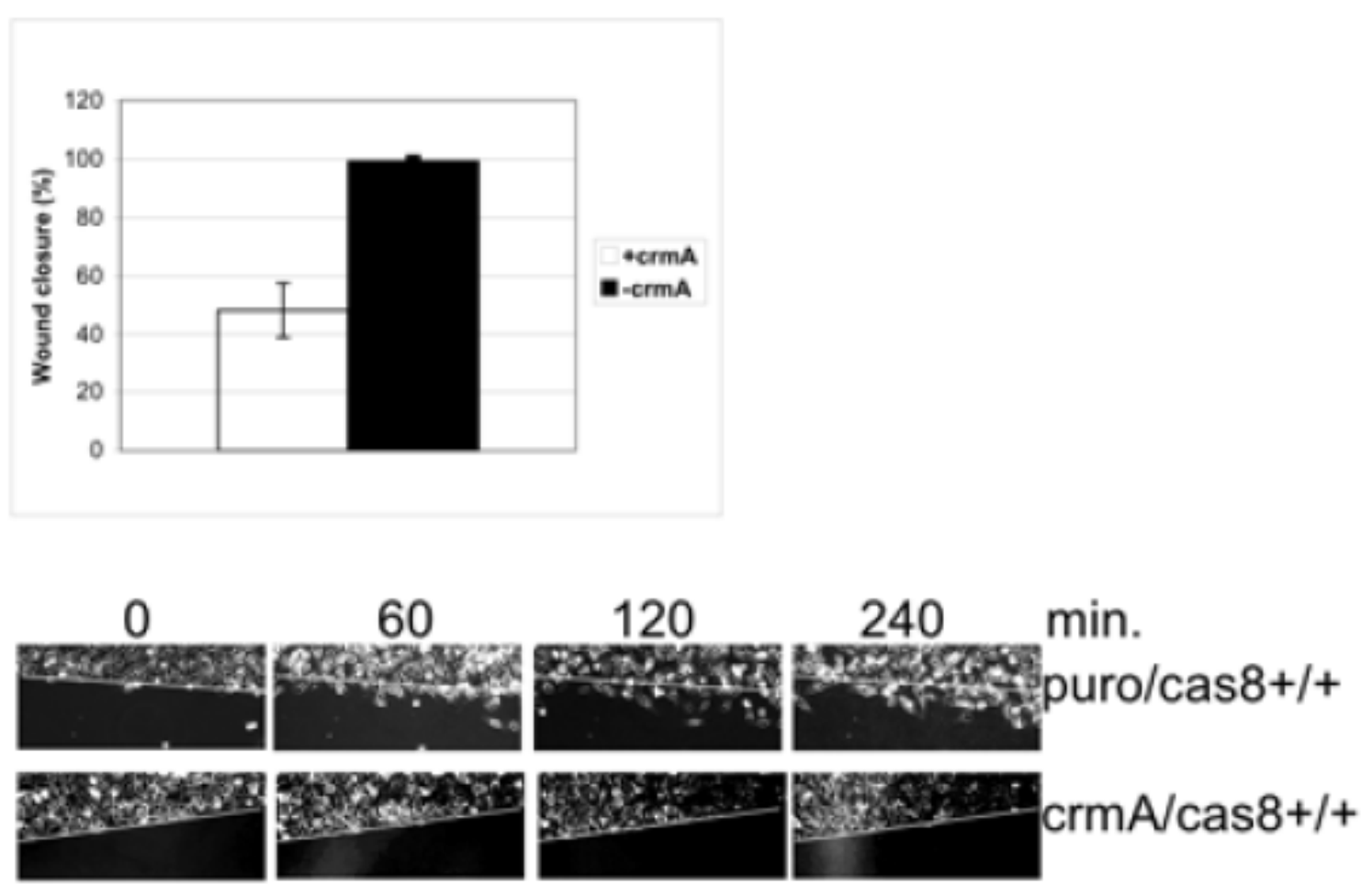

Figure 2. (C). Directed migration/chemotaxis assay, comparing MDA MB231 cells +/- CrmA, normalized for total number of adherent cells (experiment done by AD). (D) CrmA inhibits motility in WTNR6 cells (conventional wound healing assay upper) and in MEFs (conventional wound-healing assay lower). The white lines indicate the position of the original wound border in each photograph. 
E

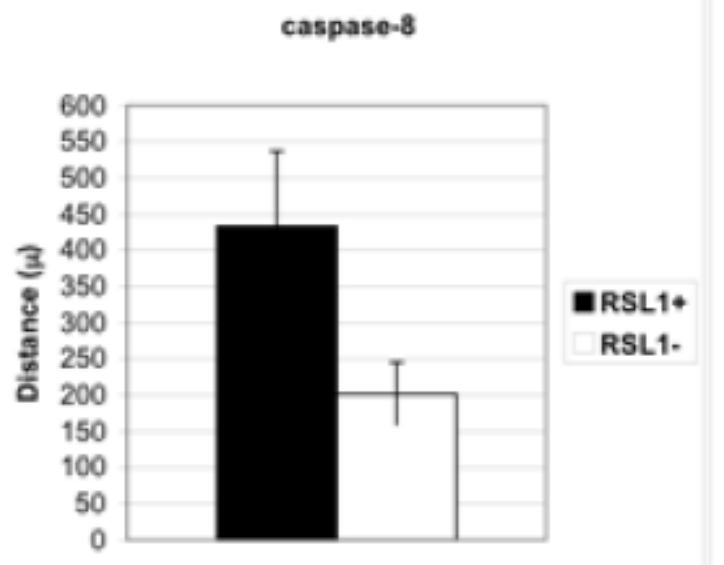

$\mathbf{F}$

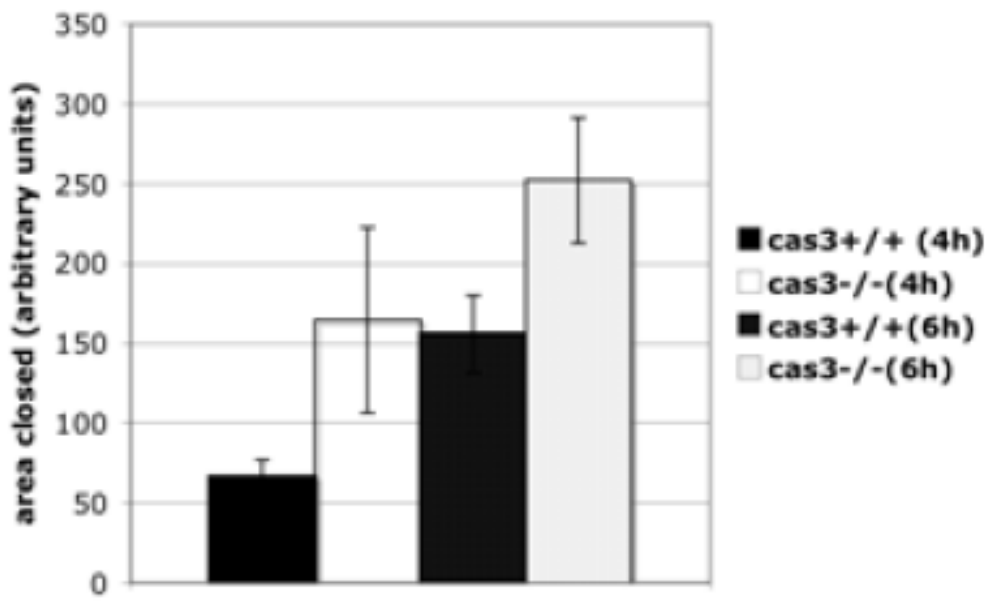

vector

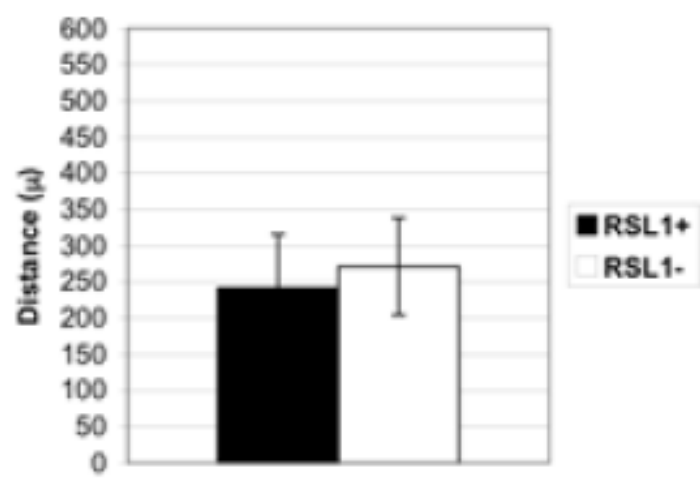

Figure 2. Caspase-8, but not caspase-3 promotes cell migration. (E) Inducible reexpression of caspase-8 stimulates motility in caspase-8-null MEFs $(p<0.001)$; the right-hand panel shows a control cell line with the rheoswitch control proteins but without caspase-8. (F) Caspase-3 does not promote cell motility. Caspase-3-- and caspase-3+++ MEFs were assayed for wound healing as in part $B$. 


\section{G}

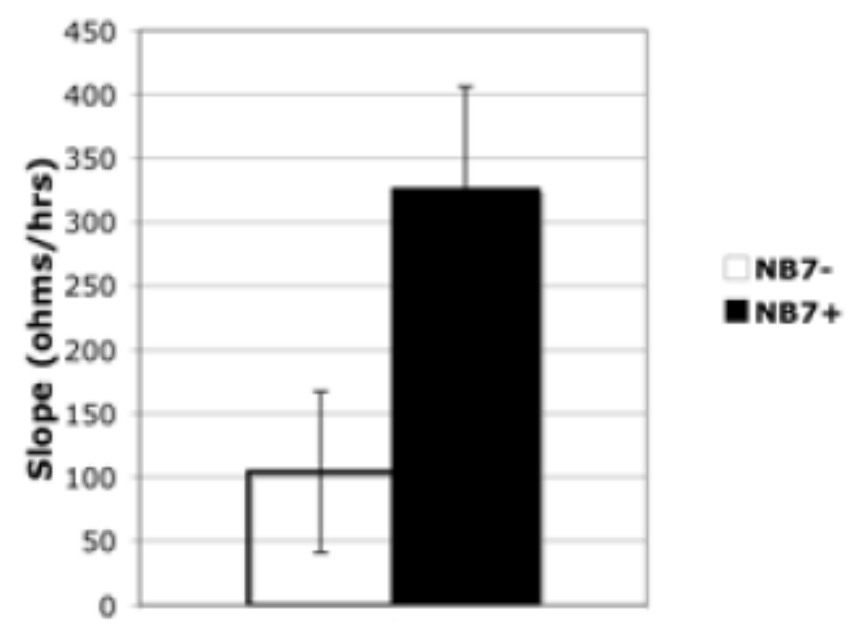

H

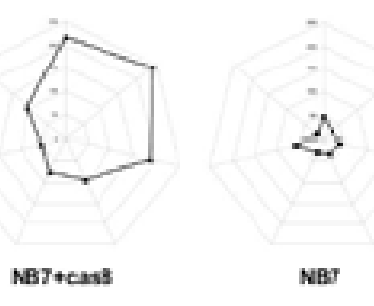

Figure 2. Caspase-8 promotes cell motility in tumor cells. (G). ECIS analysis, comparing the caspase-8-null neuroblastoma cell line NB7 ("NB7-“) vs. the caspase-8-rescued derivative cell line ("NB7+"); a graphic representation of slopes calculated from the linear part of ECIS curves (five samples for NB7-, six samples for NB7+). (H) Single-cell motility assay, comparing NB7 cells vs. NB7+caspase- 8 cells. The plot shows the distance (not direction) migrated by individual cells. 


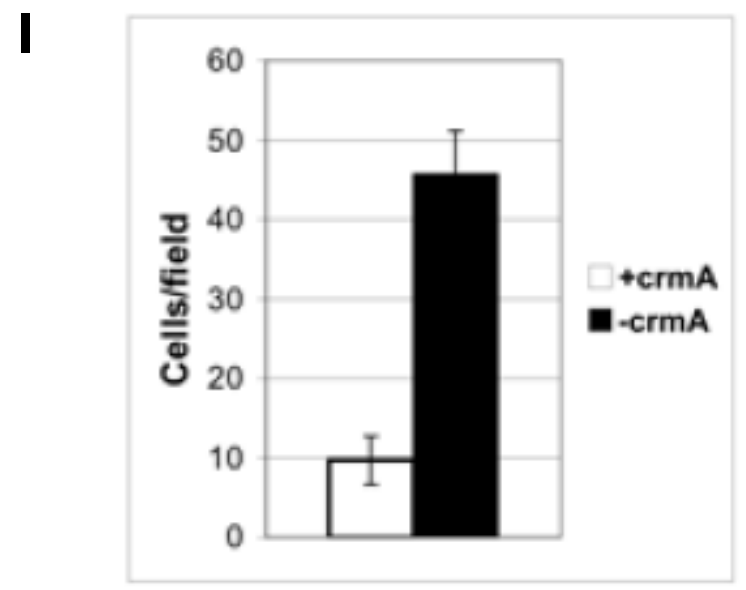

Figure 2. (I) Conventional wound healing assay, comparing the mammary carcinoma cell line MDA-MB-231 with empty vector vs. crmA. Cells migrating (four hours) into three areas of fixed size and fixed distance from original wound border were counted, averaged, and represented graphically. 


\section{Figure 3}

A
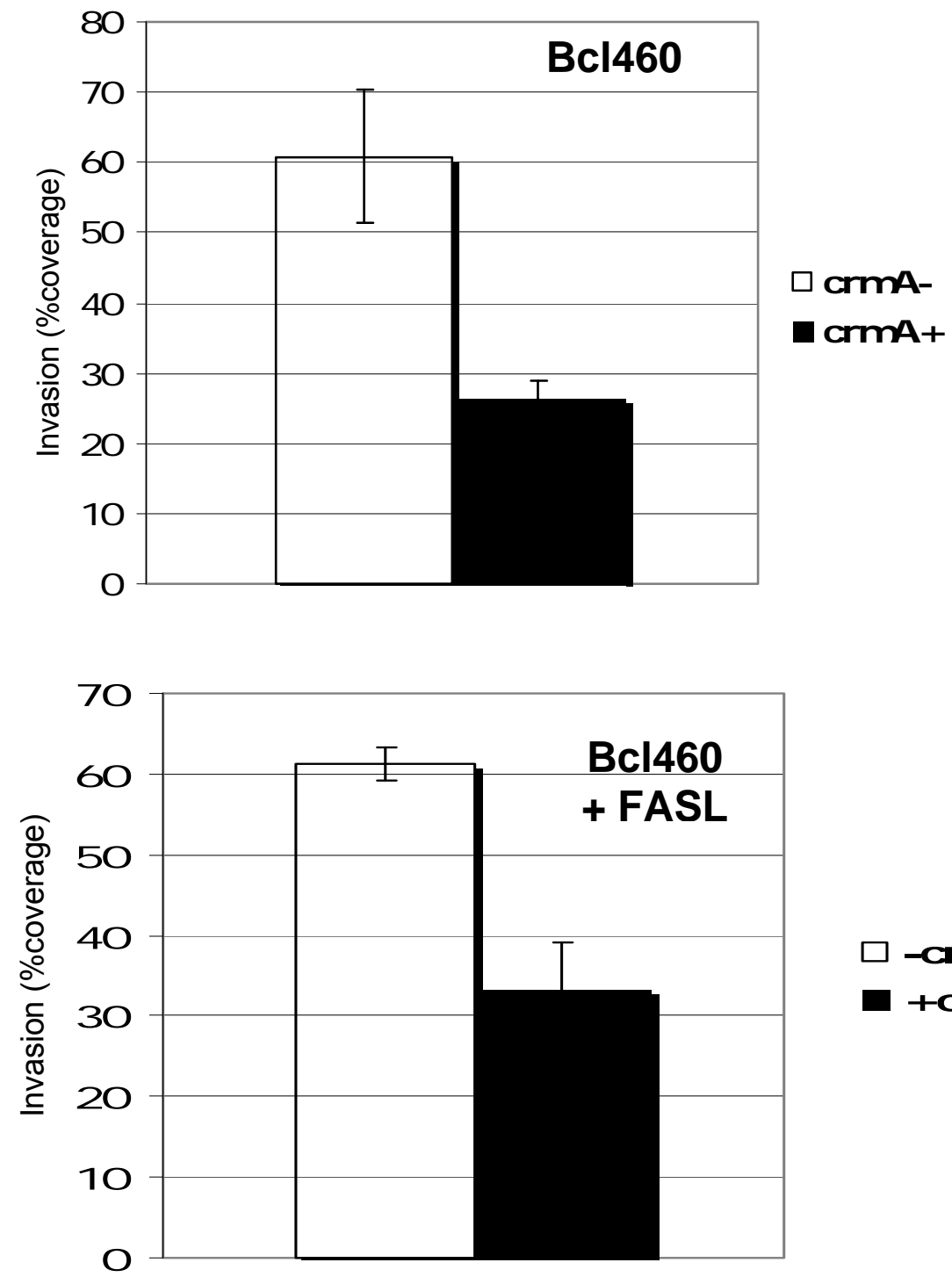

$\square$-ammA

tamma

Figure 3. Caspase-8 and FASL promote motility and invasion. (A) Caspase-8 promotes cellular invasion (upper graph), FASL stimulates invasion similarly to serum. 
B

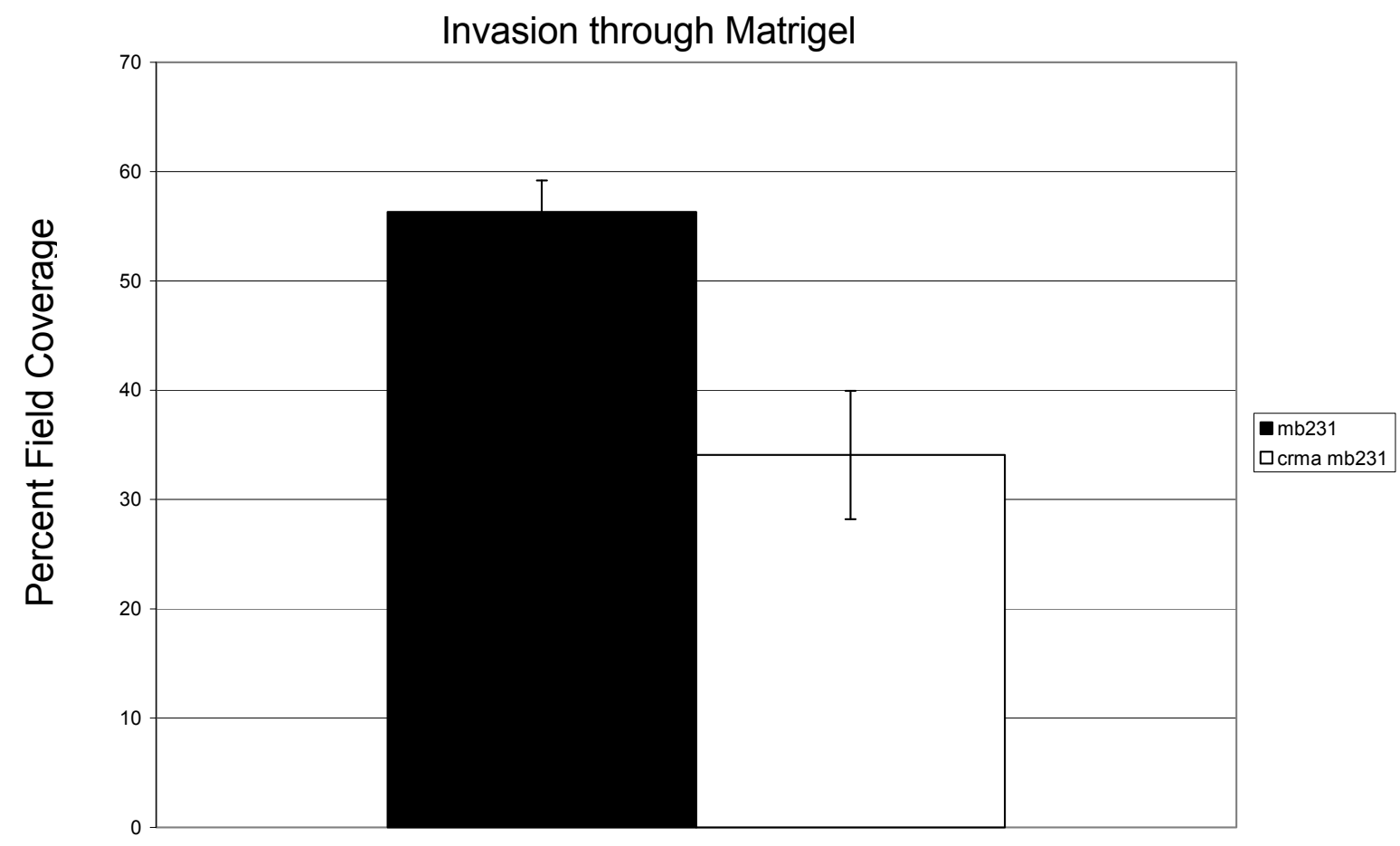

Figure 3. (B) Caspase-8 promotes invasion and transwell migration (Fig 2C) in MDA-MB231 cells. 
FASL stimulated migration

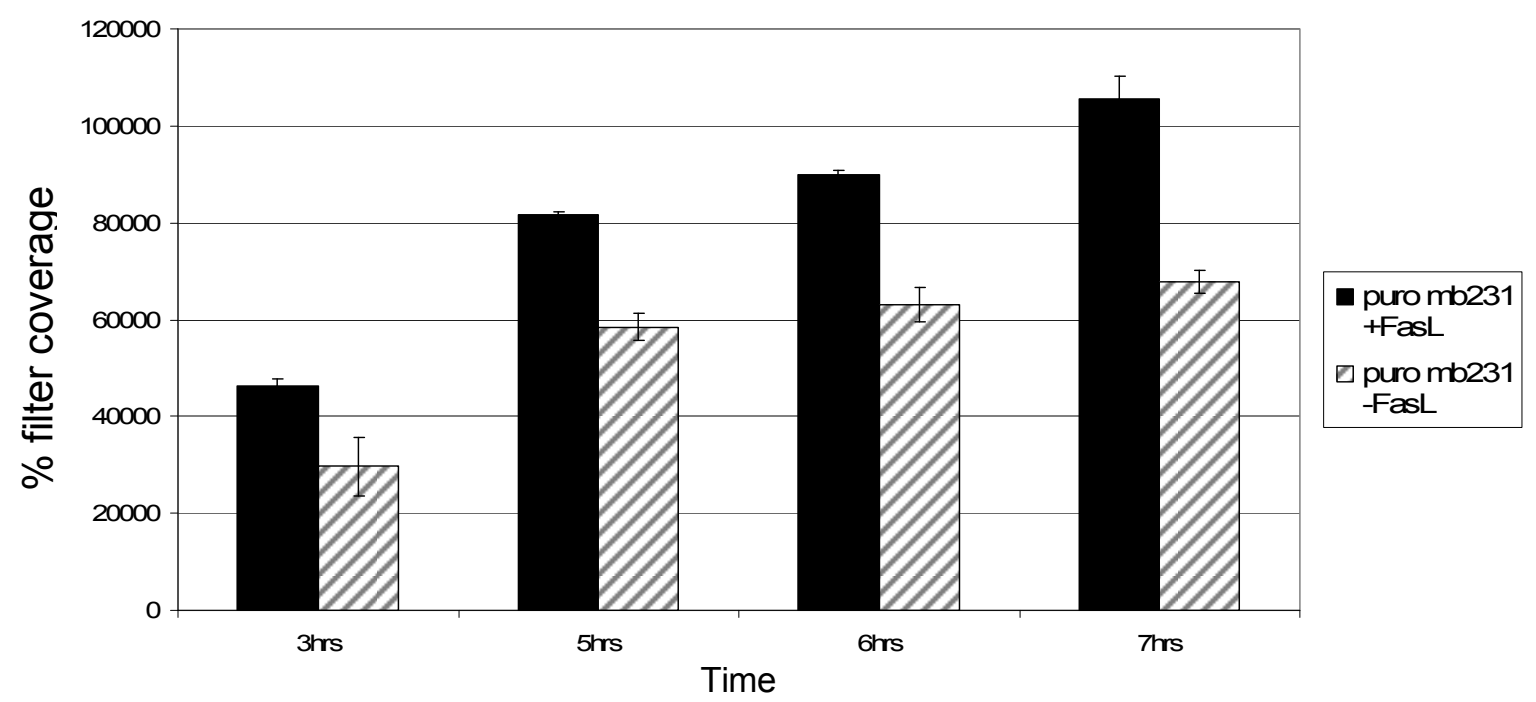

FasL stimulated motility

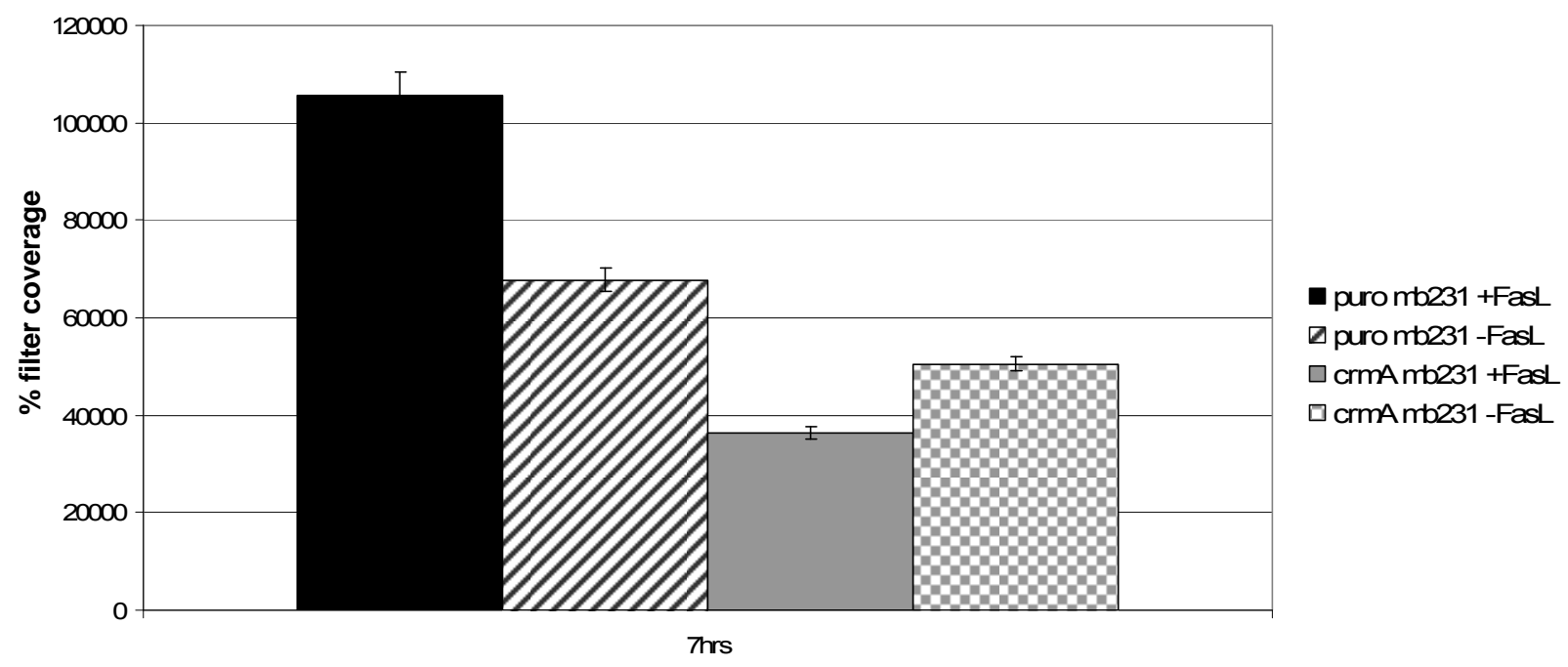

Figure 3. (C) FASL addition in these cells (MDA-MB231) also promotes cell migration (uppercaspase-8 +/- FASL in transwell migration - lower- +/- caspase-8 +/- FASL transwell migration) 


\section{Figure 4}

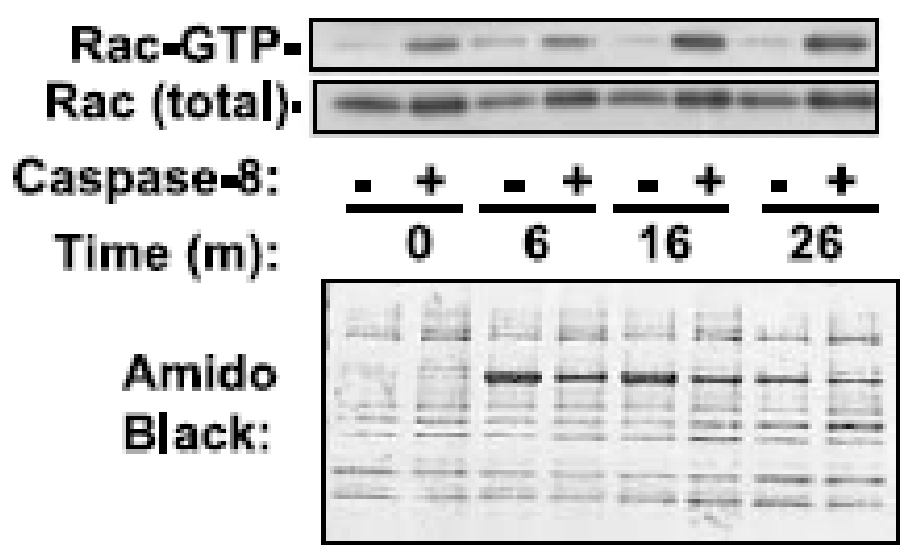

Figure 4. Caspase-8 promotes Rac activity. Caspase- $8^{-/-}$or caspase- $8^{+/+}$MEFs were assayed at the indicated times after attachment to fibronectin for activated or total rac; the amido black stained filter shows equal loading (the BSA band arises from the carrier protein used in the cell attachment-incubation buffer; the zero time sample is free of BSA because cells were immediately lysed after trypsinization.) Experiments done by SF and $\mathrm{BH}$ 


\section{Figure 5}
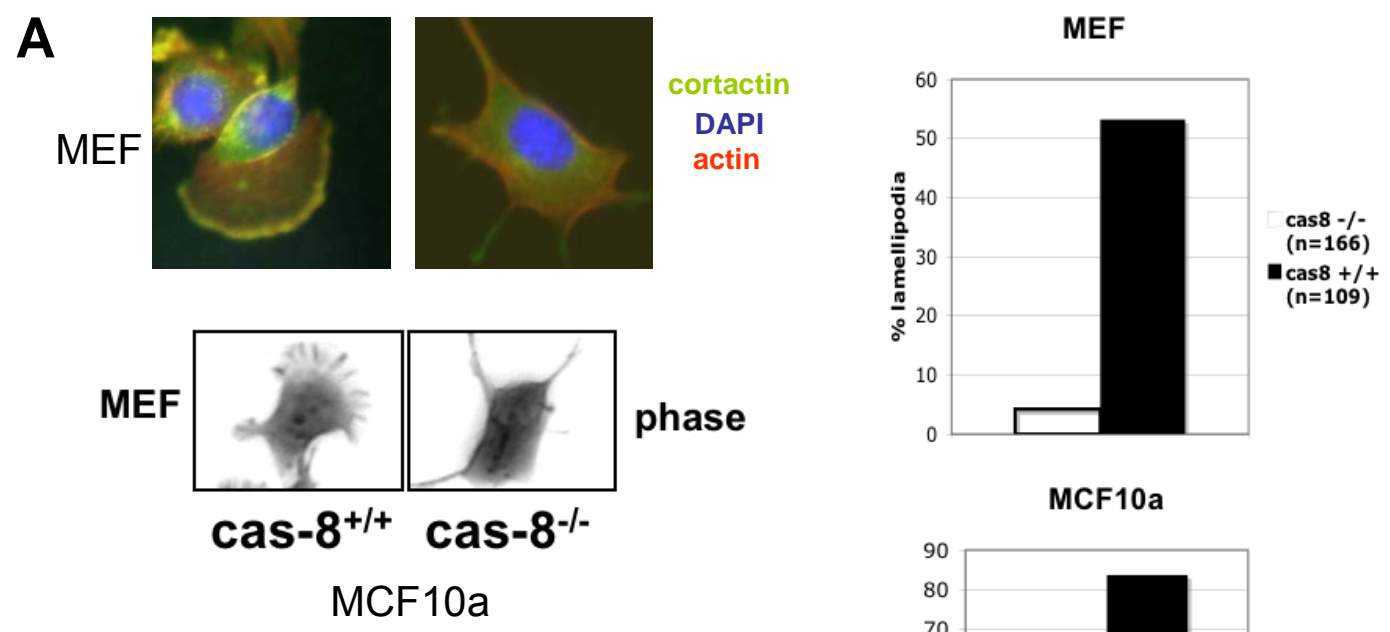

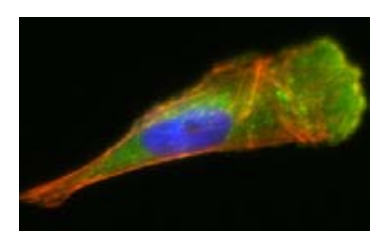

MSCV
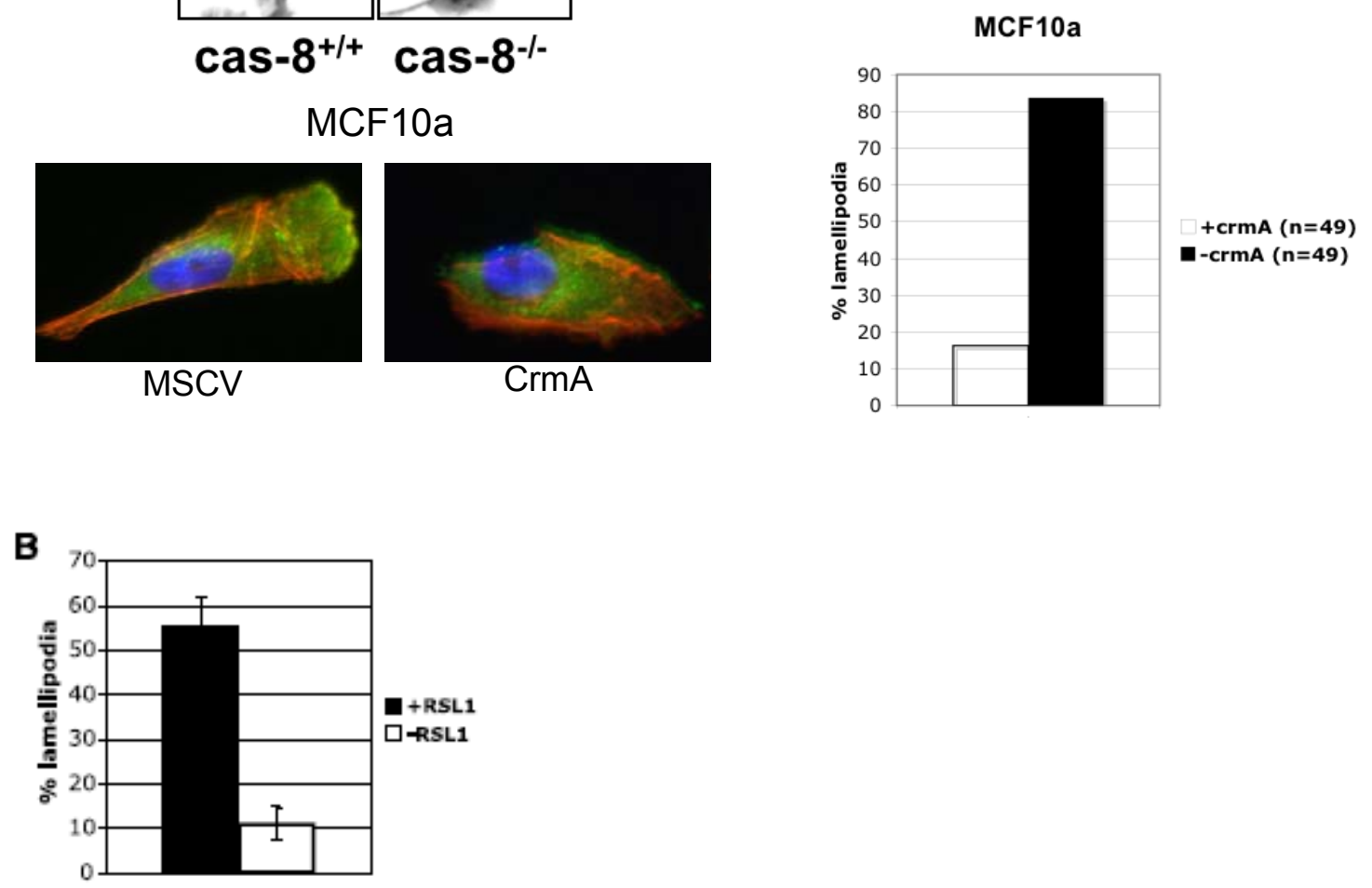

Figure 5. Caspase-8 affects cellular processes downstream of calpain activation: lamellipodial assembly, cell adhesion and normal nuclear segregation. (A). Caspase-8 promotes the accumulation of lamellipodia. (upper two rows). MCF10a cells with or without crmA were plated at low density in media containing EGF, fixed and stained with antibodies against the lamellipodia marker cortactin (green), rhodamine-phalloidin (red) and DAPI (blue). (lower row). The "attachment-control" MEFs that were assayed for chemotactic migration in figure $1 \mathrm{C}$ were stained with crystal-violet and photographed. Frequencies of lamellipodia were scored and represented graphically (the phase images were used to generate the MEF graph). ( $p$-value for MEFs: $p<0.003$, MCF10a: <0.001). (B). Caspase-8 promotes the accumulation of lamellipodia: caspase-8-inducible cell lines. 

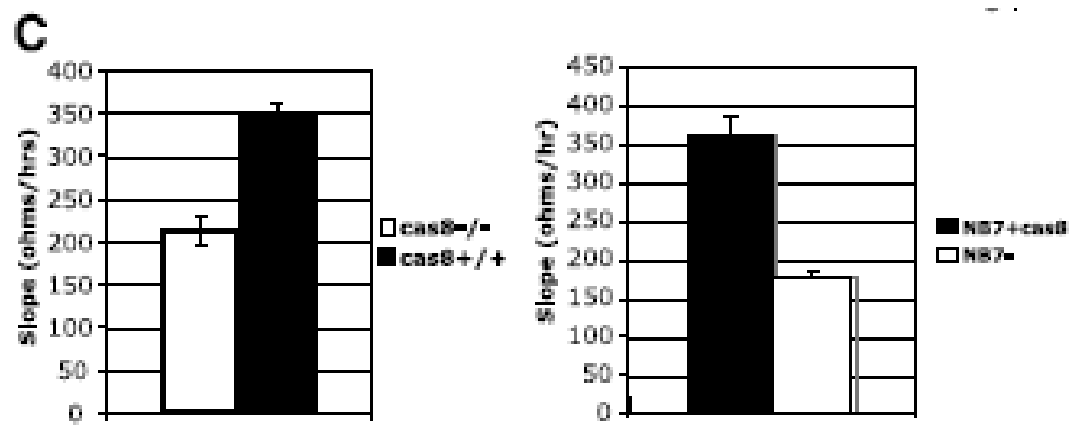

Figure 5: (C). Caspase-8 promotes cell adhesion to matrix in MEF cells and NB7 cells. 
D
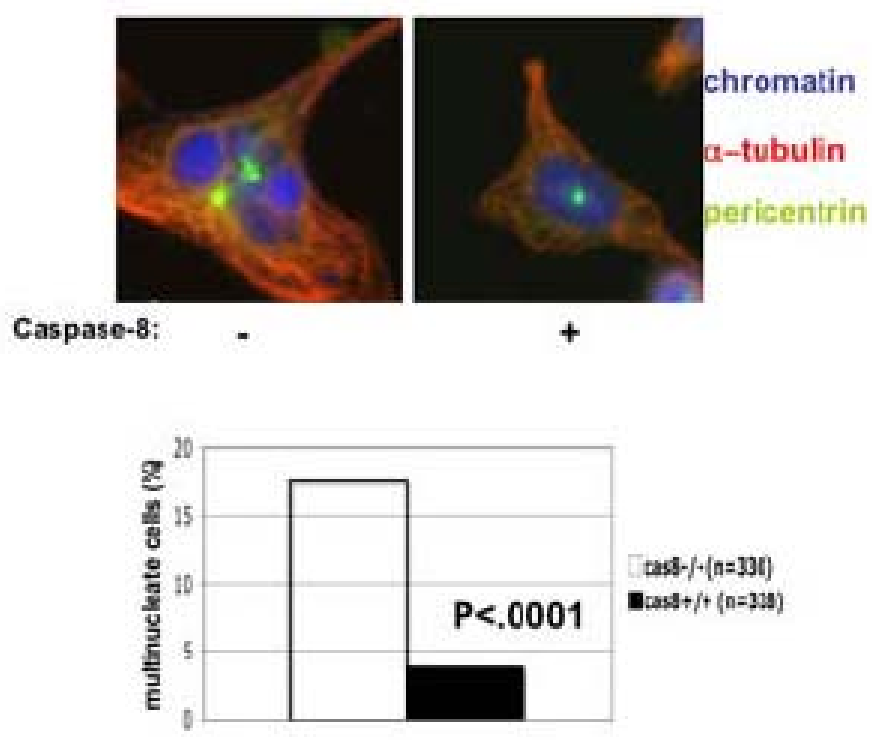

Figure 5: (D) Caspase-8-deficiency induces an elevated frequency of multinucleated and

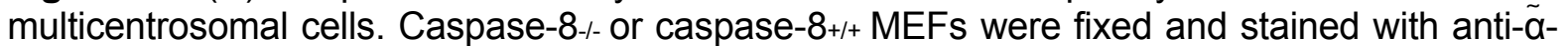
tubulin (red), anti-pericentrin (green) and DAPI (blue). The frequencies of nonmitotic cells with greater than one nucleus were scored and depicted graphically $(p<.0001)$. Examples of a multinucleate cell and a normal cell are shown below. Note that multinucleate cells often had $>2$ centrosomes. 

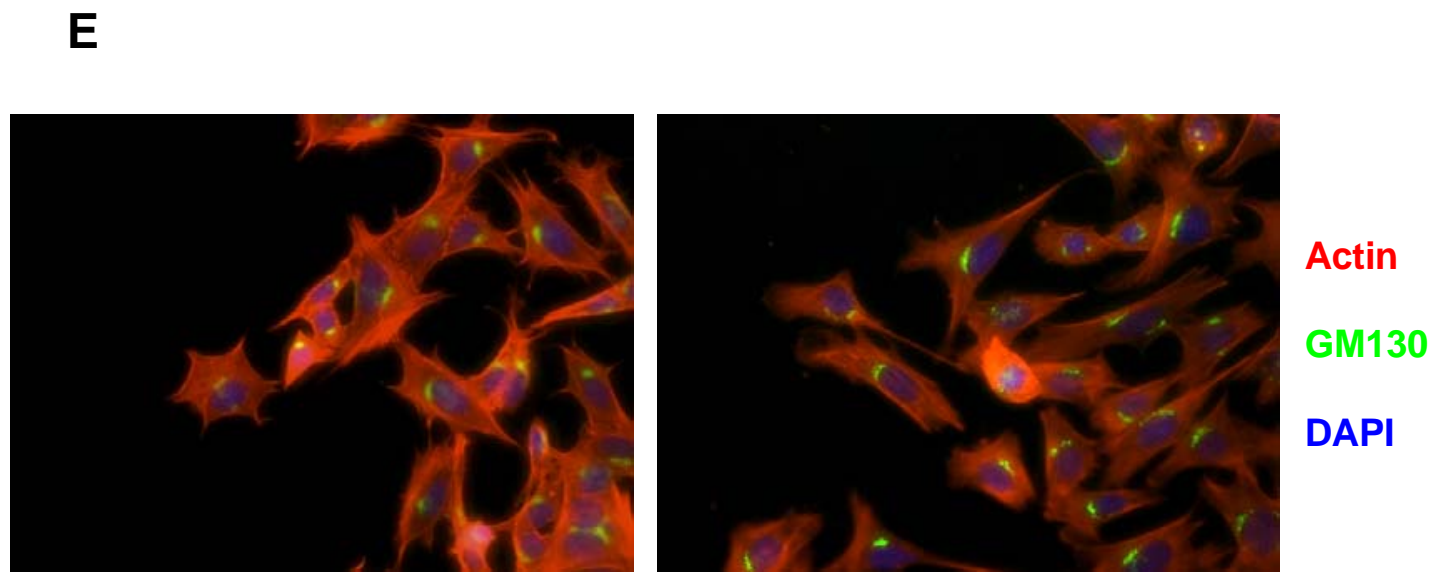

$(-)$

Figure 5: (E). Cell polarity was not altered by the lack of caspase 8. MEFs were fixed and stained with rhodamine phalloidin (red), with GM130 golgi stain (green), and DAPI (blue). 


\section{Figure 6}

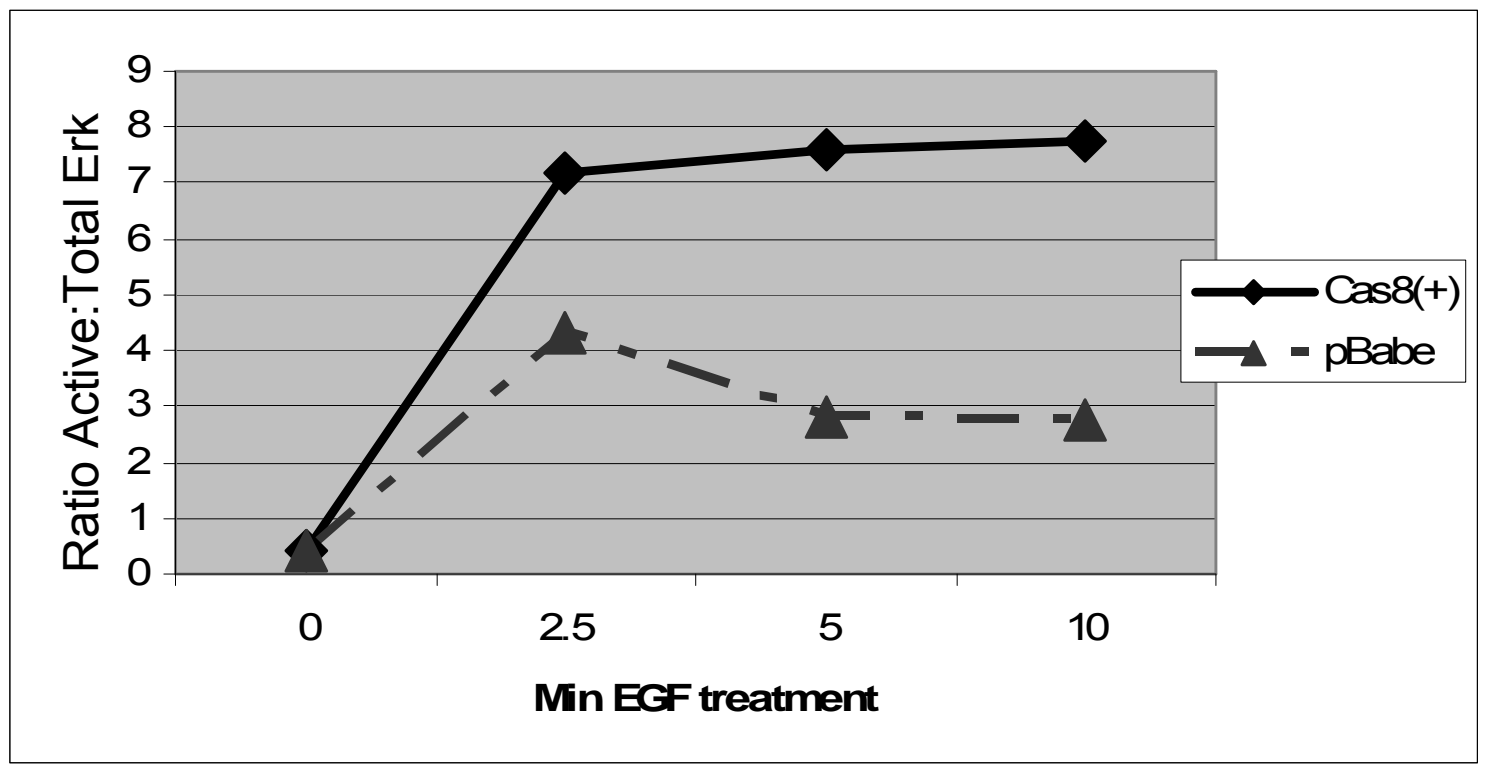

Figure 6. Caspase-8 promotes Erk activation. Cells were serum starved and treated with 10nM EGF to stimulate Erk activation. Graph represents the ratios of active to total Erk based upon densitometry of western blots of the Erk activation lysates. 


\section{Figure 7}

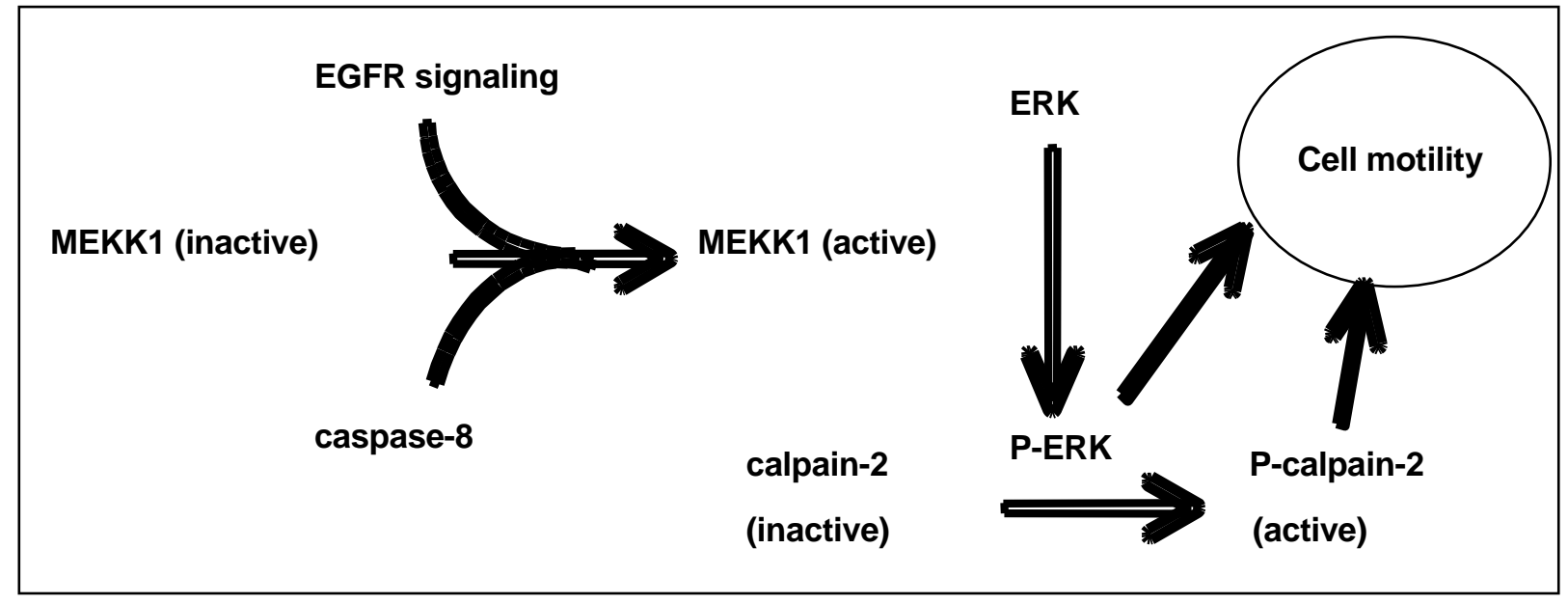

Figure 7. Proposed pathway for how caspase-8 is influencing cellular motility via an MEKK1 activation dependent mechanism that results in subsequent Erk and calpain activation. 


\section{References}

1. Gdynia, G., et al., Basal caspase activity promotes migration and invasiveness in glioblastoma cells. Mol Cancer Res, 2007. 5(12): p. 1232-40.

2. He, L., et al., Monitoring caspase activity in living cells using fluorescent proteins and flow cytometry. Am J Pathol, 2004. 164(6): p. 1901-13.

3. Yang, L., et al., Coexistence of high levels of apoptotic signaling and inhibitor of apoptosis proteins in human tumor cells: implication for cancer specific therapy. Cancer Res, 2003. 63(20): p. 6815-24.

4. Geisbrecht, E.R. and D.J. Montell, A role for Drosophila IAP1-mediated caspase inhibition in Rac-dependent cell migration. Cell, 2004. 118(1): p. 11125.

5. Barnhart, B.C., et al., CD95 ligand induces motility and invasiveness of apoptosis-resistant tumor cells. Embo J, 2004. 23(15): p. 3175-85.

6. $\mathrm{Li}$, J., et al., Caspase-11 regulates cell migration by promoting Aip1-Cofilinmediated actin depolymerization. Nat Cell Biol, 2007. 9(3): p. 276.

7. Zhao, X., et al., Caspase-3-dependent Activation of Calcium-independent Phospholipase A2 Enhances Cell Migration in Non-apoptotic Ovarian Cancer Cells. J. Biol. Chem., 2006. 281(39): p. 29357-29368.

8. Kang, T.B., et al., Caspase-8 serves both apoptotic and nonapoptotic roles. J Immunol, 2004. 173(5): p. 2976-84. 
9. Varfolomeev, E.E., et al., Targeted disruption of the mouse Caspase 8 gene ablates cell death induction by the TNF receptors, Fas/Apo1, and DR3 and is lethal prenatally. Immunity, 1998. 9(2): p. 267-76.

10. Franco, S.J. and A. Huttenlocher, Regulating cell migration: calpains make the cut. J Cell Sci, 2005. 118(Pt 17): p. 3829-38.

11. Zheng, T.S., et al., Deficiency in caspase-9 or caspase-3 induces compensatory caspase activation. Nat Med, 2000. 6(11): p. 1241-7.

12. Glading, A., et al., Epidermal growth factor receptor activation of calpain is required for fibroblast motility and occurs via an ERK/MAP kinase signaling pathway. J Biol Chem, 2000. 275(4): p. 2390-8.

13. Zhou, Q., et al., Target protease specificity of the viral serpin CrmA. Analysis of five caspases. J Biol Chem, 1997. 272(12): p. 7797-800.

14. Teitz, T., et al., Caspase 8 is deleted or silenced preferentially in childhood neuroblastomas with amplification of MYCN.[comment]. Nature Medicine, 2000. 6(5): p. 529-35.

15. Wang, L., et al., Peroxide Is a Key Mediator of Bcl-2 Down-Regulation and Apoptosis Induction by Cisplatin in Human Lung Cancer Cells. Mol Pharmacol, 2008. 73(1): p. 119-127.

16. Keese, C.R., et al., Electrical wound-healing assay for cells in vitro. Proc Natl Acad Sci U S A, 2004. 101(6): p. 1554-9.

17. Helfer, B., et al., Caspase-8 promotes cell motility and calpain activity under nonapoptotic conditions. Cancer Res, 2006. 66(8): p. 4273-8. 
18. Bialkowska, K., et al., Evidence that beta3 integrin-induced Rac activation involves the calpain-dependent formation of integrin clusters that are distinct from the focal complexes and focal adhesions that form as Rac and RhoA become active. J Cell Biol, 2000. 151(3): p. 685-96.

19. Bialkowska, K., T.C. Saido, and J.E. Fox, SH3 domain of spectrin participates in the activation of Rac in specialized calpain-induced integrin signaling complexes. J Cell Sci, 2005. 118(Pt 2): p. 381-95.

20. Dourdin, N., et al., Reduced cell migration and disruption of the actin cytoskeleton in calpain-deficient embryonic fibroblasts. J Biol Chem, 2001. 276(51): p. 48382-8.

21. Franco, S., B. Perrin, and A. Huttenlocher, Isoform specific function of calpain 2 in regulating membrane protrusion. Exp Cell Res, 2004. 299(1): p. 179-87.

22. Potter, D.A., et al., Calpain regulates actin remodeling during cell spreading. J Cell Biol, 1998. 141(3): p. 647-62.

23. Miyazaki, T., K. Honda, and H. Ohata, Requirement of Ca2+ influx- and phosphatidylinositol 3-kinase-mediated m-calpain activity for shear stressinduced endothelial cell polarity. Am J Physiol Cell Physiol, 2007. 293(4): p. C1216-1225.

24. Glading, A., et al., Epidermal growth factor activates m-calpain (calpain II), at least in part, by extracellular signal-regulated kinase-mediated phosphorylation. Mol Cell Biol, 2004. 24(6): p. 2499-512. 
25. Glading, A., et al., Membrane proximal ERK signaling is required for M-calpain activation downstream of epidermal growth factor receptor signaling. J Biol Chem, 2001. 276(26): p. 23341-8.

26. Kataoka, T., et al., The caspase-8 inhibitor FLIP promotes activation of NFkappaB and Erk signaling pathways. Curr Biol, 2000. 10(11): p. 640-8.

27. Deak, J.C., et al., Fas-induced proteolytic activation and intracellular redistribution of the stress-signaling kinase MEKK1. Proc Natl Acad Sci U S A, 1998. 95(10): p. 5595-600.

28. Kischkel, F.C., et al., Cytotoxicity-dependent APO-1 (Fas/CD95)-associated proteins form a death-inducing signaling complex (DISC) with the receptor. Embo J, 1995. 14(22): p. 5579-88.

29. Stupack, D.G., et al., Potentiation of neuroblastoma metastasis by loss of caspase-8. Nature, 2006. 439(7072): p. 95-9.

30. Bozzo, C., et al., Activation of caspase-8 triggers anoikis in human neuroblastoma cells. Neurosci Res, 2006. 56(2): p. 145-53.

31. Dobo, J., et al., Cytokine Response Modifier A Inhibition of Initiator Caspases Results in Covalent Complex Formation and Dissociation of the Caspase Tetramer. J. Biol. Chem., 2006. 281(50): p. 38781-38790.

32. Reddy, K.B., S.M. Nabha, and N. Atanaskova, Role of MAP kinase in tumor progression and invasion. Cancer Metastasis Rev, 2003. 22(4): p. 395-403. 
33. Roux, P.P. and J. Blenis, ERK and p38 MAPK-activated protein kinases: a family of protein kinases with diverse biological functions. Microbiol Mol Biol Rev, 2004. 68(2): p. 320-44.

34. Yujiri, T., et al., MEK kinase 1 interacts with focal adhesion kinase and regulates insulin receptor substrate-1 expression. J Biol Chem, 2003. 278(6): p. 3846-51.

35. Cardone, M.H., et al., The regulation of anoikis: MEKK-1 activation requires cleavage by caspases. Cell, 1997. 90(2): p. 315-23.

36. Widmann, C., et al., MEK kinase 1, a substrate for DEVD-directed caspases, is involved in genotoxin-induced apoptosis. Mol Cell Biol, 1998. 18(4): p. 2416-29.

37. Cuevas, B.D., et al., MEKK1 regulates calpain-dependent proteolysis of focal adhesion proteins for rear-end detachment of migrating fibroblasts. Embo J, 2003. 22(13): p. 3346-55. 


\title{
Chapter 3
}

\section{Pro-Caspase-8 promotes cellular migration and adhesion through interaction with the p85 subunit of PI3K}

\author{
Portions of this Chapter were published in:
}

J Senft *, Helfer, B *, S Frisch. Caspase-8 interacts with the p85 subunit of phosphatidylinositol 3-kinase to regulate cell adhesion and motility. Cancer Rearch 2007; 67: 11505-11504

${ }^{*}$ co-first-authors 


\section{Abstract}

Cell migration plays an important role in tumor cell invasion and metastasis. We reported that caspase-8 contributes to cell migration and adhesion, a novel nonapoptotic function of an established apoptotic factor. In this study, we report that pro-caspase-8 is capable of restoring cell migration/adhesion to caspase-8-null cells, establishing the first biological function of a pro-caspase. The catalytic activity of caspase-8 was not required for cell motility. Stimulation of motility with epidermal growth factor induced the phosphorylation of caspase-8 on tyrosine-380 and the interaction of caspase-8 with the p85a subunit of phosphatidylinositol 3-kinase. Tyrosine-380 was required for the restoration of cell motility and cell adhesion in caspase-8-null cells, demonstrating the importance of the caspase-8-p85 interaction for these nonapoptotic functions. These results suggest that caspase-8 phosphorylation converts it from a proapoptotic factor to a cell motility factor that, through tyrosine-380, interacts with $\mathrm{p} 85$, an established cell migration component. 


\section{Introduction}

In chapter 2, we reported that caspase-8 promotes cell migration, adhesion, invasion, and Rac activation in normal and tumor cell lines [1], processes important in tumor cell metastasis [2]. Certain tumors, such as neuroblastoma and small-cell lung cancer, involve the loss of caspase-8 expression $[3,4]$, but many tumor types retain and often over-express caspase-8 (Figure 1). Thus, caspase-8 may contribute to metastasis in cell types that are resistant to apoptosis due to alterations in numerous genes, other than caspase-8. This mechanism could have important ramifications for cancer therapy.

Recently, caspase-8 has been shown to be phosphorylated by src in a manner that prevents its autocleavage during apoptosis [5]. The site of src phosphorylation is at tyrosine residue 380 , which is located in the caspase-8 linker region spanning amino acids $374-384$ (Figure 8). In the proapoptotic processing of caspase-8 this is the first sequence of caspase- 8 that is cleaved in its autoactivation [6]. Thus, the only form in which caspase- 8 would be phosphorylated would be as a full length procaspase. Our laboratory hypothesized that the phorphorylated form of pro-caspase-8 might interact with signaling proteins involved in cell migration, a major focus of this chapter.

As reviewed in chapter 1 , one important cell migration factor that interacts with tyrosine phosphorylated partners is phosphatidylinositol 3-kinase (PI3K). P85, the regulatory subunit of $\mathrm{PI} 3 \mathrm{~K}$, is affected by the binding of tyrosine phosphorylated proteins to its $\mathrm{SH} 2$ domains, as well as by src-mediated phosphorylation [7]. The p85 
subunit is able to interact with many proteins involved with the promotion of migration, such as EGFR, RhoGAP1, Ras, FAK, c-Src, [8-12]. p85 has also been shown to interact with Rac and cdc42 and potentially contribute to cytoskeletal dynamics [1315]. Although PI3K activity is known to activate Rac by promoting PIP3 and stimulating Rac-GEF activity, the direct interaction of Rac with p85 might play an additional role in Rac regulation, which has not been addressed. The phosphorylation of caspase-8 at Y380, within the sequence YELM, makes it a candidate for SH2 domain interaction.

Procaspase- 8 retains catalytic activity [16] and may be able to promote migration through cleavage of various unidentified substrates; alternatively it may interact with $\mathrm{SH} 2$ domains of migration promoting proteins, such as $\mathrm{PI} 3 \mathrm{~K}$, via phosphorylated $\mathrm{Y} 380$ to promote cell migration. The phosphorylation of caspase-8 also supplies an attractive switch mechanism between its promigratory, non-apoptotic functions, and its role in apoptosis.

In this study, we show that $Y 380$-phosphorylated pro-caspase-8 interacts directly with the p85 subunit of $\mathrm{PI} 3 \mathrm{~K}$, promoting cell adhesion and motility. 


\section{Experimental Procedures}

Cell lines. NB7 cells were obtained and cultured as described previously (2). Stable derivatives expressing caspase-8 mutants were constructed by packaging the appropriate retrovirus construct in the vector pBABE-puro in gp2+293 cells in the presence of pAmpho (Invitrogen), infection of viral stock, selection with $0.5 \mu \mathrm{g} / \mathrm{mL}$ puromycin, and western blotting of mixed populations; similar expression levels were obtained for all constructs used in this study (data not shown). MTLN3s were kindly provided by Dr Scott Weed. A431 epidermal carcinoma cells were obtained from American Type Culture Collection and maintained in MEM containing $10 \%$ fetal bovine serum, penicillin, streptomycin, and glutamine.

DNA constructs, transfections, and pull downs. All caspase-8 constructs were made by PCR using catalytically inactive caspase-8 (C360A) template kindly provided by G. Salvesen (Burnham Institute). Mutations were made by the use of the Stratagene Quick-Change mutagenesis system, according to manufacturer's instructions, in the vector pNEBRX1 (New England Biolabs) and mutant constructs were sequenced, prior to being subcloned into the BamHI-Sall site of pBABE-puro. To create S-tag constructs, a double-stranded oligonucleotide encoding the S-tag sequence (forward: GATCCAAAGAAACCGCTGCTGCGAAATTTGAACGCCAGCA CATGGACTCGTAGGC; reverse: GGCCGCCTACGAGTCCATGTGCTGGCGTTCA AATTTCGCAGCAGCGGTTTCTTTG) was synthesized and three-way ligated with a HindIII-BamHI PCR fragment of caspase-8 and pcDNA3.1 linearized with HindIII and Notl. Caspase-8-S-tag/pBABE-puro retroviruses were constructed by subcloning the 
Pmel-Pmel fragments from the pcDNA3.1 constructs into pBABE-puro that was linearized with SnaBI.

A431 cells were serum-starved in Dulbecco's Modified Eagle's Medium containing penicillin, streptomycin and glutamine overnight. After treatment for four minutes with $200 \mathrm{ng} / \mathrm{ml}$ EGF (Calbiochem), cells were lysed by scraping in in $25 \mathrm{mM}$ Tris $\mathrm{pH} 7.6 / 150 \mathrm{mM} \mathrm{NaCl} / 25 \mathrm{mM} \mathrm{NaF/1 \%}$ TritonX100/1 mM sodium orthovanadate/1X Roche complete protease inhibitor cocktail. Sodium deoxycholate was added to the lysate to give $0.5 \%$ final concentration, and, after pre-clearing at 13,200 rpm for 12 minutes, SDS was added to $0.1 \%$ final concentration. For S-tag pulldown experiments, S-protein agarose bead suspension that had been pre-equilibrated in lysis buffer containing $10 \mathrm{mg} / \mathrm{ml}$ bovine serum albumin and $10 \mathrm{mg} / \mathrm{ml}$ ovalbumin and washed three times prior to use, were added to the lysates. After incubation with the beads at 4 degrees for 1.5 hours with rotation and three washes, samples were boiled in SDS sample buffer and analyzed on western blots. Antibodies for western blotting were from the following sources: caspase-8 (mAb 3-1-9): BD Biosciences; HA (mAb HA.11): Covance, Inc.; p85 (pAbs 06-195 and 06-496): Upstate Biotechnology.

Rac activation assay. Cells were incubated overnight in RPMI with $0.5 \%$ FBS, detached by treatment with TrypLe Express (Invitrogen) and neutralized by 10 -fold dilution in serum-free medium, followed by centrifugation. The cells were then resuspended in serum-free RPMI containing 3\% BSA and plated onto a series of 6 well plates precoated with $15 \mu \mathrm{g} / \mathrm{mL}$ fibronectin (Calbiochem) and blocked with $3 \%$ 
BSA. At the indicated times, cells were scraped into $0.6 \mathrm{~mL}$ cold lysis buffer [25mM HEPES, $150 \mathrm{mM} \mathrm{NaCl}, 10 \mathrm{mM} \mathrm{MgCl} 2,5 \%$ glycerol, 1\% Igepal + complete Roche protease inhibitors, EDTA-free] and precleared. Ten micrograms of bacterially expressed GST-PAK (CRIB domain) protein were added and the samples were incubated on ice for 1 hour, followed by addition of $30 \mu \mathrm{L}$ of a $50 \%$ slurry of glutathione-Sepharose (Pharmacia, Piscataway, $\mathrm{NJ}$; equilibrated in lysis buffer) and further incubation for 1 hour in the cold room on a rotation device. Beads were washed thrice in lysis buffer and analyzed on Western blots for Rac using monoclonal antibodies (mAb) from BD PharMingen, peroxidase-labeled antimouse antibody (Zymed), and Pierce West Pico chemiluminescence kit (Pierce, Rockford, IL).

Adhesion and motility assays. Adhesion measurements using electrical cell substrate impedance sensing (ECIS) and motility measurements using single-cell motility were done as described previously ([1] chapter 2). Summarized briefly:

(Single cell motility assay): Cells were plated onto fibronectin coated dishes at low density for 2 hours in the absence of serum, stimulated with $10 \%$ serum containing $\mathrm{CO}_{2}$ independent media (Invitrogen), and recorded by videomicroscopy for 8 hours. The paths traveled by individual cells were traced and the total distance traveled by each cell was computed using the Axiovision software (interactive measurement module) and depicted radially using Excel.

(Electrical cell-substratum impedance sensing): Eight well ten-electrode electrical cell-substratum impedance sensing (ECIS) arrays (Applied Biophysics, Troy, NY; www.biophysics.com) were pre-coated with $15 \mu \mathrm{g} / \mathrm{mL}$ fibronectin. Cells were the 
trypsinized, spun down, and resuspended in serum free media. $1 \times 10^{5}$ cells were applied per well. As cells attach to the array, the impedance increases due to the cells attaching and spreading onto the electrode. Adhesion was then monitored at 45 $\mathrm{kHz}$ frequency over the indicated time course; adhesion was carried out in duplicate or triplicate. Data were exported to Excel for the calculation of slopes.

Akt and EGFR phosphorylation assays. NB7 cells +/- caspase 8 were serumstarved overnight $(0.5 \%$ serum $)$ at $\sim 75 \%$ confluence. Cells were then treated with 10ng/mL EGF for $0,2.5,5,10,35$, and 60 minutes, washed in cold PBS and lysed in SDS sample buffer. Lysates were analyzed on parallel western blots for phospho-Akt (Cell Signaling rabbit polyclonal Phosphoserine-473-Akt) and total Akt (Cell Signaling Akt pAb) or with phospho-EGFR (Zymed) or total EGFR (BD Transduction Labs). Scans were quantitated by the use of Image $\mathrm{J}$ software.

Recombinant protein generation and in-vitro interactions. S-tag caspase-8 was generated in pET29a and GST-p85 in pGEX. Recombinant protein isolation was done as described previously [17]. Briefly, overnight cultures of bacteriaprep were inoculated into $500 \mathrm{~mL}$ of pre-warmed LB, incubated for two hours and $0.15 \mathrm{mM}$ IPTG added and incubated for an additional 2 hours. Bacteria is then isolated, resuspended in protease inhibitor containing PBS and lysed in $10 \mu \mathrm{M}$ DTT, $1 \%$ Triton-X 100, and $1 \mathrm{mg} / \mathrm{mL}$ lysozyme, sonicated and then pelleted. The supernatant is then incubated stag beads or with glutathione sepharose, that was prequillibrated with PBS, for two hours at $4^{\circ} \mathrm{C}$. Beads were then isolated, washed and resuspended in inhibitor 
containing PBS in the case of caspase- 8 or eluted with $20 \mathrm{mM}$ glutathione and dialyzed against PBS in the case of GST-p85.

Caspase- 8 was phosphorylated by mixing beads with src kinase buffer (25mM HEPES pH7.5, 0.01\% Brij35, 0.03\% beta-mercaptoethanol, and $10 \mathrm{mM} \mathrm{MgCl}_{2}+/-50$ $\mu \mathrm{M}$ ATP + Pierce protease inhibitor cocktail) and $0.5 \mu \mathrm{g}$ of active Src (Upstate) for 30 min on a wheel at $37^{\circ} \mathrm{C}$. Beads were then washed in $1 \mathrm{X}$ interaction buffer ( $25 \mathrm{mM}$ Tris $\mathrm{pH} 8,150 \mathrm{mM} \mathrm{NaCl}, 0.1 \%$ Triton-X 100, 10\% glycerol, $1 \mathrm{mg} / \mathrm{mL}$ BSA, and 0.5mM DTT) then mixed with GST-p85 or GST (5 or $10 \mu \mathrm{g}$ ) for 3 hours at $4^{\circ} \mathrm{C}$. Beads were then isolated, washed with interaction buffer and lysed in $2 x$ LSB $+10 \%$ betamercaptoethanol and western blot probing for p85 N-terminal SH2 domain (Upstate).

Immunofluorescence: Cell grown on coverslips were treated with 100nM EGF or 100nM Rhodamine labeled EGF and then fixed with 4\% paraformaldehyde in PBS. Cells were then permeabilization with $0.1 \%$ TritonX100 for five-minutes. Cells were stained with anti-HA mouse monoclonal antibody (Covance), Primary antibody incubation was followed by Alexa-488 (Pierce) and rhodamine phalloidin when R-EGF was not used. Coverslips were then mounted with Vectashield containing DAPI (Vector Labs). Images were collected on a Zeiss Axiovert 200M microscope using AxioVision software. 


\section{Results}

\section{Caspase-8 enhancement of cell adhesion/motility is dependent on tyrosine-380, but independent of catalytic activity or autoproteolytic}

processing. Chapter 2 demonstrated that caspase-8 promoted EGF stimulated cell migration and adhesion in MEF as well as in other normal and cancer cell models [1]. The lack of caspase-8 activation/cleavage products suggested that pro-caspase-8 might be performing these functions (data not shown). An uncleavable mutant of caspase-8 (DA, D374A/D384A [6]) was examined for its effects on both cellular adhesion and migration (Figure 2). The caspase-8 DA mutant was expressed in neuroblastoma cells and was found to promote both adhesion and migration as effectively as did wild-type caspase-8 (Chapter 2). A catalytically inactive mutant, C360A, also promoted cellular adhesion and migration (Figure 2). These results indicated that caspase-8 performs these functions independently of catalytic activity and auto-cleavage.

Src phosphorylates caspase-8 on Y380 and prevents its proapoptotic processing [5]. Accordingly, we confirmed that EGF stimulation results in the phosphorylation of caspase-8 (Figure 3B [5]). Since Y380 phosphorylated caspase-8 is only present in procaspase-8, non-phosphorylated mutants (Y380F) were tested in the context of both uncleavable and catalytically inactive caspase-8. When Y380 was mutated to phenylalanine, caspase-8 failed to promote both cellular adhesion and migration indicating that this residue is crucial for the promotion of its non-apoptotic functions. These results suggest that caspase-8 may interact with other signaling proteins 
through phospho-Y380 to elicit its effects, being that the promotion of adhesion and migration is independent of catalytic activity.

Caspase-8 interacts with the p85 subunit of PI3K. Phosphorylation of caspase-8 on Y380 warranted the investigation of potential SH2 domain interactions. To test potential interactions with $\mathrm{SH} 2$ domain proteins, a $\mathrm{SH} 2$ domain array (Panomics) was screened with a phosphorylated peptide spanning the Y380 sequence (Figure $3 \mathrm{~A}$, experiment performed by SF). Several proteins were detected via this approach but few failed to show an interaction in co-transfection experiments (supplemental figure 2 of [18]). Of particular interest was the C-terminal SH2 domain of the p85 subunit of PI3K because interaction with p85/PI3K could explain the promigratory/adhesion results of procaspase-8.

To determine an optimal phosphorylation time for caspase-8 upon EGF stimulation, caspase-8 isolation (s-tagged C360A caspase-8 construct pull down) was analyzed in the context of stable A431, epidermal carcinoma, cells. Five minutes of EGF stimulation was optimal for caspase-8 phosphorylation (Figure 3B).

To further examine a caspase-8 p85 interaction, A431 cells were subjected to stagged C360A caspase-8 pull downs after EGF stimulation. A time course of EGF stimulation shows that caspase-8 interacts with the p85 subunit of PI3K (Figure 3C). This interaction was also verified via endogenous caspase-8 immunoprecipitation by p85 (Senft et al Figure 3D [18]). Moreover, the in-vitro phosphorylation of 
recombinant caspase- 8 by src followed by incubation with recombinant p85 indicates that phospho-caspase-8 interacts directly with p85, as implied by the Panomics SH2 array (Figure 3D).

The caspase-8-p85 interaction affects Rac activation. The activation of Rac following cell-matrix adhesion was partially dependent on caspase-8 (Chapter 2 [1]). The importance of $\mathrm{Y} 380$ in both cellular adhesion and migration warranted the examination of its role in Rac activation. Upon cellular adhesion, neuroblastoma cells expressing Y380 or F380 caspase-8, were analyzed for Rac activation. Y380 caspase-8 promoted Rac activation (Figure 4A) more efficiently than F380 caspase8, supporting the results in Figure 2. One potential mechanism for the activation of Rac through p85 is the stimulation of PI3K activity. Utilizing phosphorylation of Akt on serine-473, an indicator of PI3K activity, Akt phosphorylation was not, affected by caspase-8 after EGF stimulation (Fig. 4B) or serum re-addition (data not shown). It is therefore more likely that caspase- 8 affects Rac activation mediated by p85 through an alternative mechanism that remains to be elucidated (see Discussion).

Caspase-8 at the cell periphery, a potential EGFR signaling complex. Caspase-8 could also interact with p85 in a complex involving EGFR. Colocalization imaging was done for caspase-8 and EGFR in A431 cells that were made to express low levels of HA-caspase-8 (C360A). These images showed relocalization of a fraction caspase-8 from a perinuclear staining pattern to membrane ruffles after EGF treatment (Fig 5A). To further examine this phenomenon HA-caspase-8 was examined in MTLN3 cells 
due to their ready formation of lamellipodia upon EGF stimulation. MTLN3 cells again showed a striking relocalization of caspase-8 to membrane ruffles after EGF treatment (Fig 5B). Due to the colocalization of caspase-8 with rhodamine labeled EGF, it is possible that caspase-8 interacts with protein complexes along with EGFR, or with EGFR directly to influence p85 and potention Rac activation. EGFR phosphorylation on Y1086 was not influenced by the expression of caspase-8 (Fig 5C), nor was phosphorylation of Y1068 (data not shown) making it unlikely that capase-8 is affecting EGFR signaling/activation. While these are preliminary experiments, caspase-8 s-tag pull downs of EGFR were variable, but occasionally showed coprecipitation, warranting another look at this potential interaction.

Caspase-8 D302 influences cellular adhesion and migration. Two additional forms of caspase-8 were examined to determine if they influenced caspase-8's ability to promote cell migration and/or cellular adhesion. A single nucleotide polymorphism $(\mathrm{SNP}), \mathrm{D} 285 \mathrm{H}$, is associated with reduced susceptibility to breast, lung, gastric, colorectal, and cervical cancers [19-21]. This polymorphism did not affect cell adhesion or cell motility (Fig 6). We also examined $\mathrm{D} 302 \mathrm{H}$ because this aspartate residue is adjacent to a p85 binding consensus sequence (YXXMD), suggesting that additional p85 binding sites on caspase-8 may exist (see [18] Figure 3B Y380F 293T analysis). Mutation of the aspartate reside (D302H) diminished caspase-8's ability to promote cellular motility and adhesion (Fig 7). These data suggested that the 298YXXM301 motif may be able to bind p85, an experiment that has yet to be addressed. 


\section{Discussion and Future Studies}

Caspase-8 promoted cell migration (Chapter 2). This study expands upon this phenomenon demonstrating that both pro-caspase-8 and a catalytic-inactive mutant of caspase-8 promoted cell migration and adhesion. Importantly, the Y380 residue of caspase-8 was required for these processes, a residue that is lost upon the first autocleavage event of caspase-8 in apoptosis [6].

EGF treatment induces the phosphorylation of caspase-8 on Y380, possibly mediated by c-src [5]. We have shown that this phosphorylation is crucial for caspase-8 interaction with the p85 regulatory subunit of PI3K. Initial screening of phosphorylated caspase-8 with a Panomics SH2 array suggested direct interaction of caspase-8 with other proteins in a phosphorylation $\mathrm{SH} 2$ domain dependent manner. This interaction led us to believe that caspase- 8 could be involved in a signaling complex to promote cellular adhesion and motility. The C-terminal SH2 domain of p85 interacted with Y380-phosphorylated caspase-8 [18]. In vitro interaction studies confirmed that phospho-caspase-8 was interacting directly with p85. A subsequent publication showed an interaction of phosphorylated caspase-8 with c-src in vitro, a result that is not inconsistent with ours' [22]. This report confirmed that the catalytic activity of caspase- 8 was not required for cell migration and the $\mathrm{Y} 380$ residue was required.

We also examined the role of a caspase-8 $\mathrm{D} 285 \mathrm{H}$ in cell migration. D285H is a SNP of caspase-8 that is associated with decreased incidence of several cancers, did not influence cell migration/adhesion. Interestingly, a six-nucleotide polymorphic variant 
has been found in the promoter region of caspase-8 [21]. This polymorphism decreases caspase-8 expression and also results in decreased cancer incidence. This paired with the maintenance of caspase- 8 in many tumors indicates that caspase-8 may contribute to tumor progression through its interaction with the p85 subunit of $\mathrm{PI} 3 \mathrm{~K}$.

A third paper dealing with caspase-8 and the promotion of adhesion agrees that caspase-8 does not require catalytic activity to promote cellular adhesion [23]. They examined caspase-8's ability to interact with src at the cell periphery. They attributed this localization to an interaction of src with the death effector domains of caspase-8. We have not examined the role of the death effector domains of caspase-8 in cellular adhesion and migration, but we noted that, in contrast with Finlay et al [23], the DED domains of caspase-8 were found to aggregate and mislocalize [24]. Also, the DED domains alone were found to induce cell senescence, rendering any effects on adhesion and motility difficult to interpret [25].

Caspase-8's ability to interact with p85 and yet activate Erk and Rac leads to the inquiry of other proteins involved in a complex with caspase-8. This question is one being examined by our lab presently. The caspase-8:p85 complex could interact directly with Rac to influence its activity or could interact with other proteins to convey this promotion [26]. Further in-vitro interactions of caspase-8, p85 and Rac are being explored. Proteomic analysis for novel caspase-8 interactions are being conducted, using stable cell lines expressing caspase-8-FLAG-S-tag constructs and sequential 
purification on FLAG and S-protein affinity resins. Thus far ANT2 (Adenine Nucleotide Translocase) has been isolated as an interactor. We anticipate that caspase- 8 and ANT2, which is a mitochondrial co-factor for Bax activation $[27,28]$, contributes to apoptosis but is unlikely to be involved in mechanisms addressed here.

The interaction of phosphorylated caspase- 8 with p85 provides one mechanism for the effects of caspase- 8 on cell adhesion, migration, and Rac activation. It is important to note, however, that additional mechanisms undoubtedly exist. Mouse caspase-8, which lacks Y380, can also stimulate cell motility [1]. Although the mouse homologue would not be predicted to be effective in the human cell system, there may be human cell contexts in which other mechanisms involving catalytic activity play a more significant role, mechanism such as Erk activation mediated by calpain (Chapter 2). In this connection, mouse and human caspase-8 both enhance Erk activation (Chapter 2 and unpublished data BB and SF). Furthermore, both mouse and human caspase-8 contain $\mathrm{Y} 334$, a site predicted to be phosphorylated by mass proteomics [29]. This tyrosine, Y298, and other unidentified phosphorylation sites, may be able to interact with $\mathrm{SH} 2$ domains in a similar manner to $\mathrm{Y} 380$.

We propose that caspase-8 utilizes an apoptosis vs. migration switch mechanism. This mechanism (Figure 8 ) utilizes caspase-8 being phosphorylated by src or upon EGF treatment to block apoptotic autoprocessing [5]. We have shown here that this phosphorylation is important in the promotion of cell migration. This mechanism would therefore demonstrate the coordination of caspase-8 phosphorylation blocking 
apoptosis and allowing the interaction of caspase- 8 with p85 to promote cell migration, switching caspase-8 from an apoptotic to a migratory factor.

Caspase-8 could regulate PI3K-mediated cell migration [30-32] through at least two different pathways. First, caspase-8 could affect PI3K catalytic activity (reviewed in chapter 1). Second, caspase-8 could affect the activation of Rac or cdc42 that is directly complexed with p85 $[14,26]$. One scenario for this involves the p85 interaction with Rac/cdc42 via Abi1 [26]; however we could not reproduce Abi1 mediated Rac activation in our system (data not shown). The ability of caspase-8 to relocalize upon EGF stimulation does not allow us to exclude the possibility that caspase- 8 is promoting localized PI3K activation. The lack of effect of caspase- 8 on Akt phosphorylation does not support a localized PI3K activation, but rather supports a Rac/CDC42 activating mechanism. Details of this mechanism are being elucidated currently. 


\section{Figure 1}
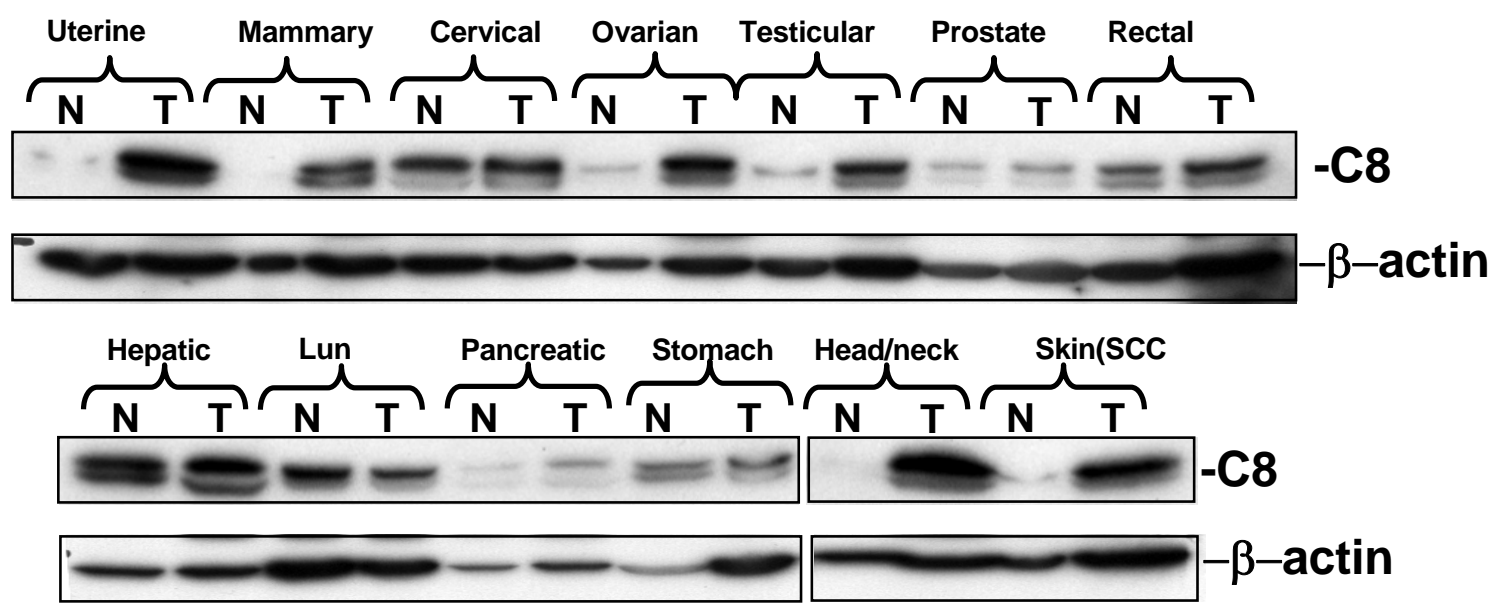

Figure 1: Caspase-8 expression is maintained in human tumors. Two western blots (GBiosciences) containing alternating lanes of total protein (50 micrograms) from the indicated matched normal or tumor samples were probed with antibodies against caspase- 8 or $\tilde{\beta}$-actin. 


\section{Figure 2}

A
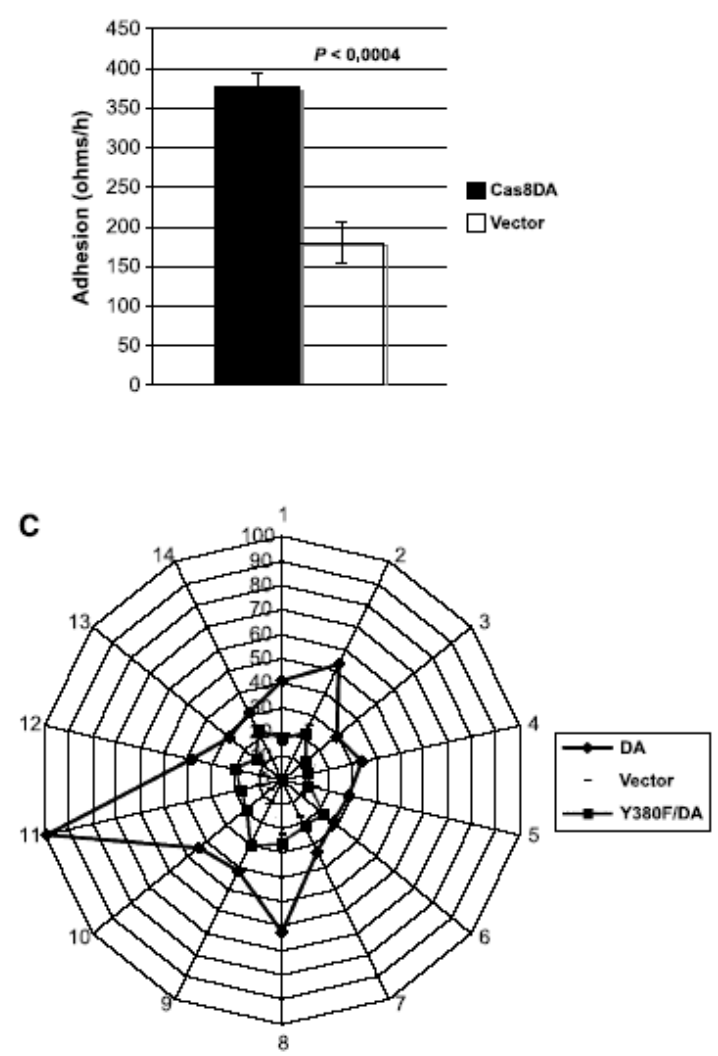

B

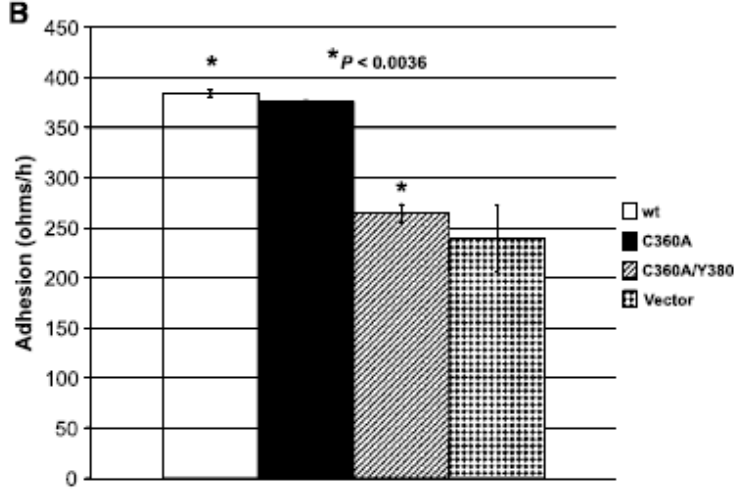

D

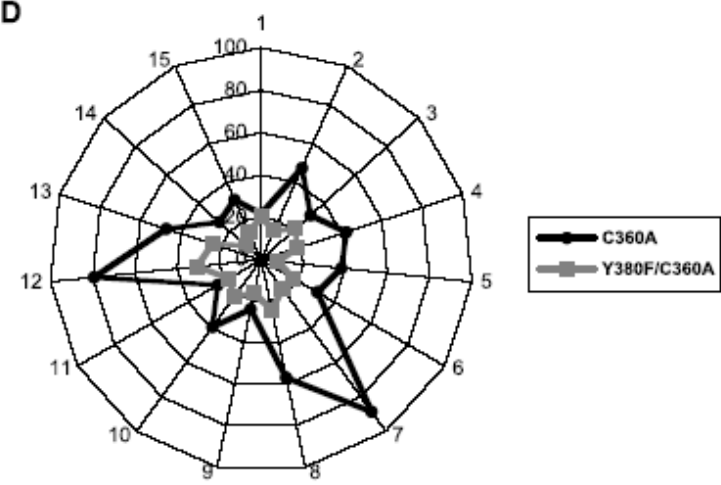

Figure 2: Caspase-8 enhancement of cell adhesion/motility is dependent on tyrosine380 , but independent of catalytic activity or autoproteolytic processing. For these experiments, the caspase-8-null cell line NB7 was infected with retroviral caspase-8 expression constructs as indicated and assayed for motility or adhesion. A, pro-caspase-8 rescues adhesion. Cells were assayed for kinetics of adhesion to fibronectin using ECIS; the slopes of the linear portion of the ECIS tracing are plotted; note that the fold stimulation of adhesion is similar to that of wild-type caspase-8 reported previously (2). Cas8DA, the uncleavable caspase-8 mutant, D374A/D384A; vector, pBABE-puro. B, the catalytic activity of caspase- 8 is not required and the phosphorylation site $Y 380$ is critical for cell adhesion. An ECIS adhesion assay comparing the wild-type (wt), catalytically inactive (C360A), or the phosphorylation site- defective mutant of inactive (Y380F/C360A) caspase-8 is shown. $P$ values for the difference between the indicated samples are shown. C, pro-caspase-8 promotes cell motility, which is dependent on Y380. A single cell-motility assay is presented as a radial plot indicating distance (not direction) of migration. DA, the uncleavable mutant D374A/D384A; Y380F/DA, the phosphorylation site-defective/uncleavable double mutant; vector, $p B A B E$-puro. $D$, inactive caspase-8 promotes cell motility, which is dependent on Y380. C360A, catalytically inactive caspase-8; Y380F/C360A, the phosphorylation sitedefective/catalytically inactive double mutant caspase-8. 


\section{Figure 3}

A

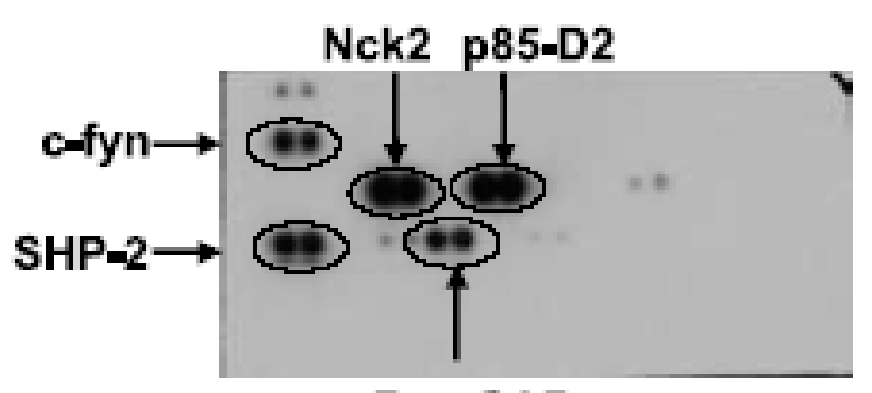

Ras-GAP

Figure 3. Caspase-8 interacts with p85. A, a phosphorylated peptide spanning caspase-8 Y380 reveals candidate interaction partners including p85. Experiment was performed by SF. 


\section{B}

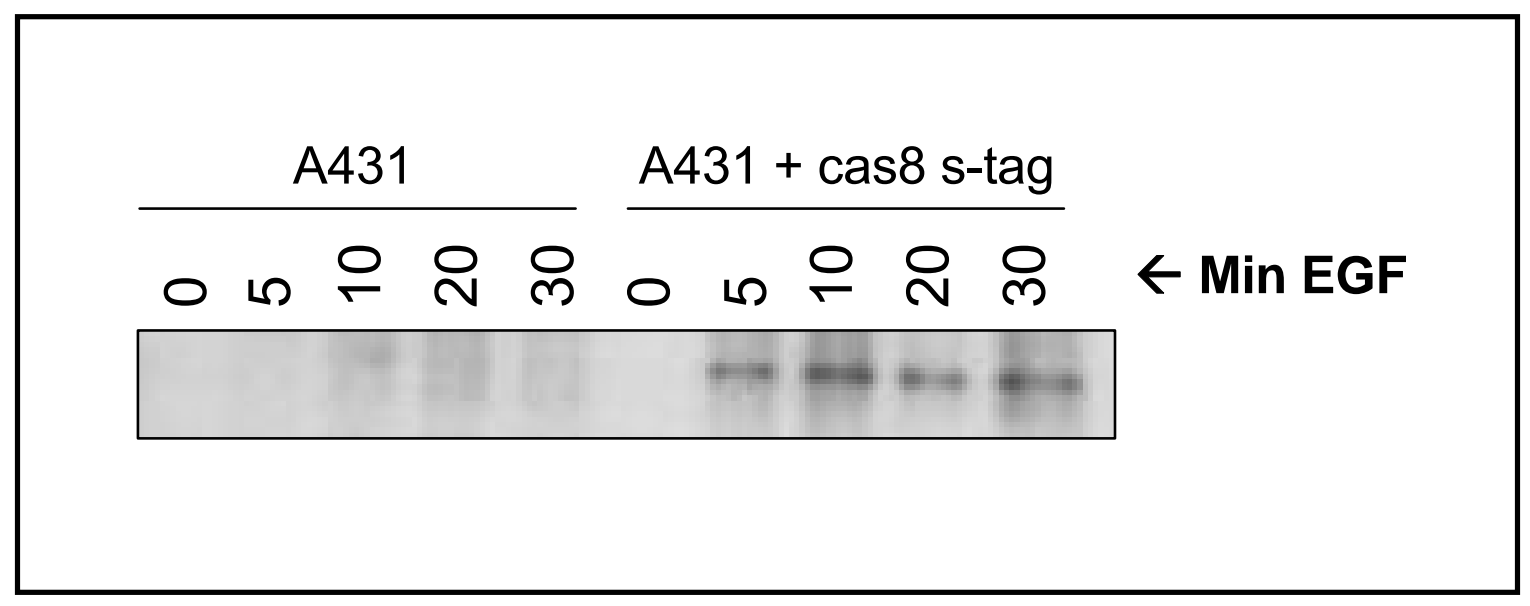

Figure 3. Caspase-8 interacts with p85. B. Phosphotyrosine $4 \mathrm{G} 10$ analysis of caspase-8 stag pulldowns on A431 cells +/- s-tag caspase-8 for detection of caspase-8 phosphorylation. 


\section{C}

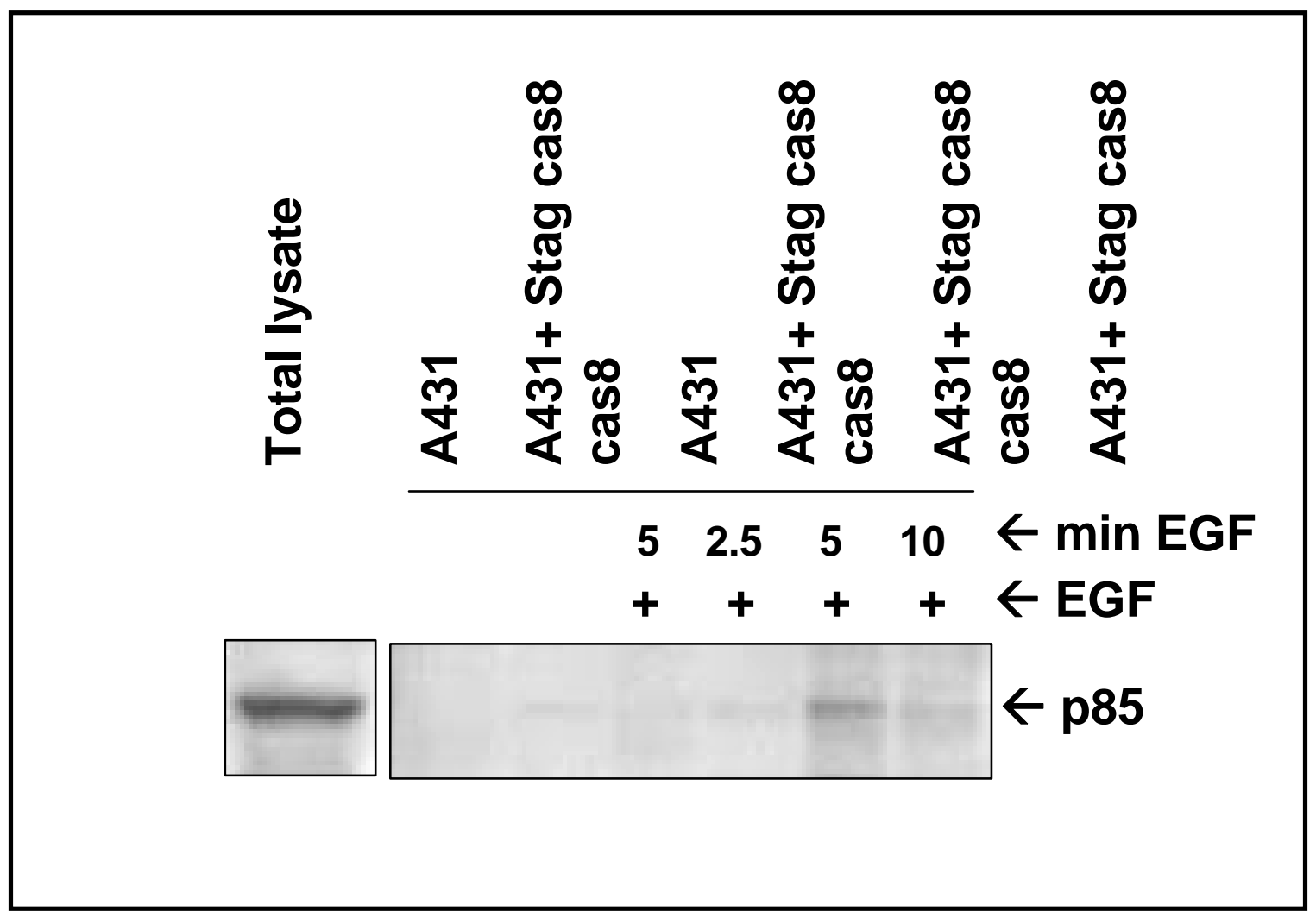

Figure 3. Caspase-8 interacts with p85. C. A431 s-tag pulldowns probed for p85. Stable A431 + caspase-8 s-tag (presumably phosphorylated caspase-8 when treated with EGF) interacted specifically with p85 when compared to control A431 cells treated with EGF. 
D

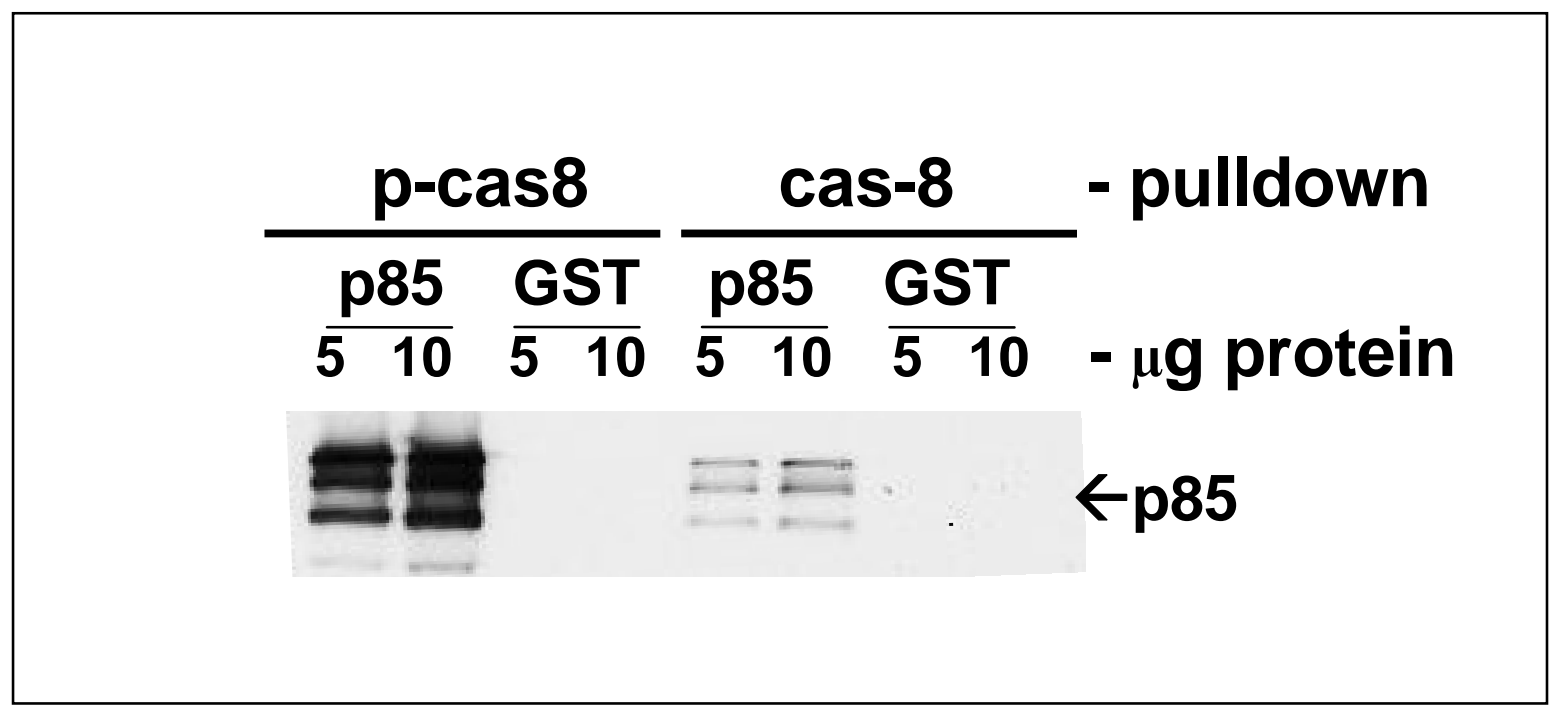

Figure 3. Caspase-8 interacts with p85. D, Direct in-vitro interactions of caspase-8 +/phosphorylation with GST-p85 but not with GST alone. 


\section{Figure 4}
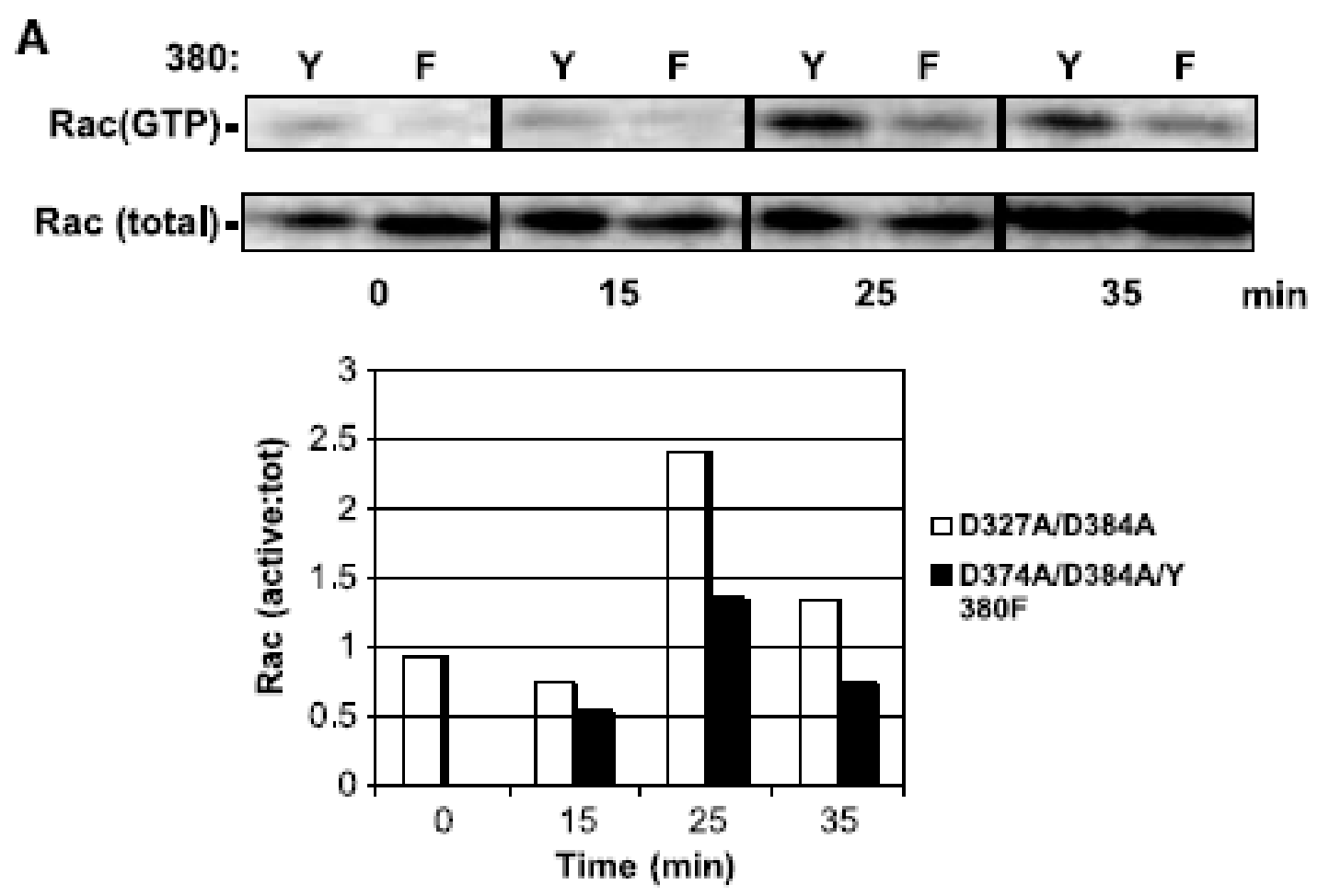

Figure 4: Caspase-8-p85 interaction contributes to Rac activation but does not enhance Akt activation. A, caspase-8-p85 interaction contributes to Rac activation. NB7 cells rescued with uncleavable caspase-8 containing Y380 (Y) or F380 (F) were trypsinized, attached to fibronectin-coated dishes for the indicated times, and lysates were assayed for total or activated Rac. Bottom, a plot of activated Rac to total Rac ratios derived by densitometry; an additional experiment produced similar results. The average fold increase of Y380 versus $\mathrm{F} 380$ at 25 min was $1.67 \mathrm{~F} 0.14$. D374A/D384A, uncleavable caspase-8 mutant; D374A/D384A/Y380F, uncleavable/phosphorylation site-defective caspase-8 mutant. 
B

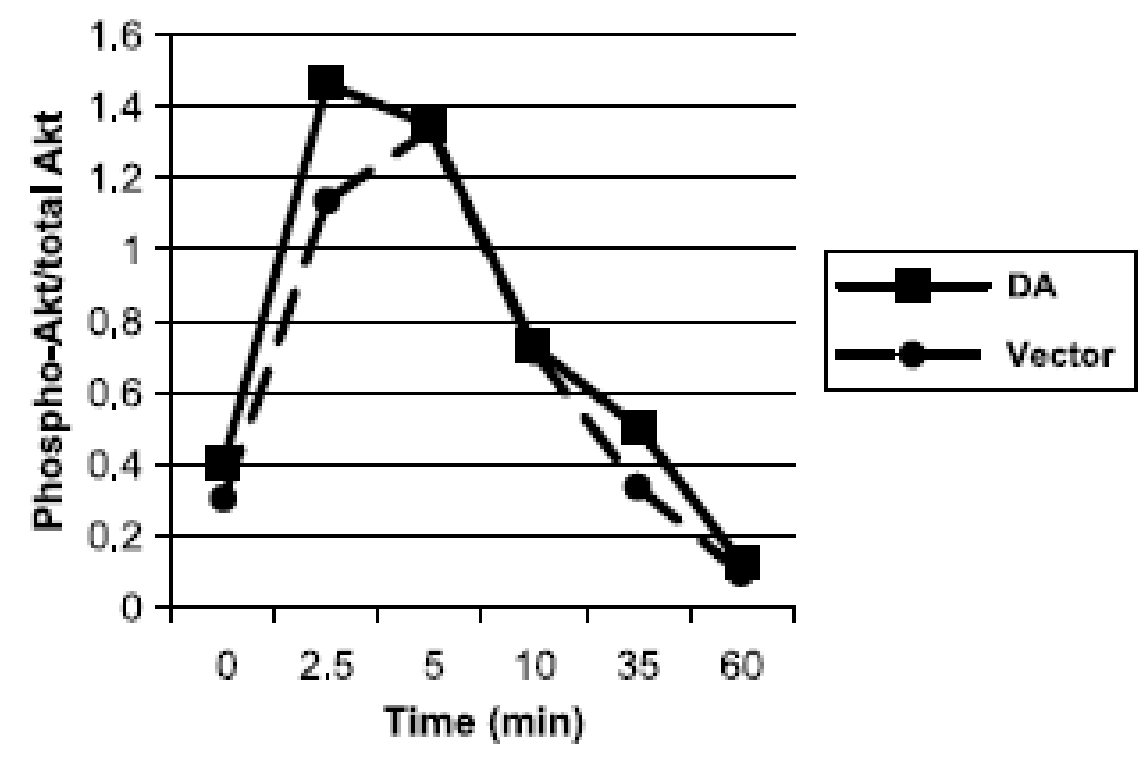

Figure 4: Caspase-8-p85 interaction contributes to Rac activation but does not enhance Akt activation. B, caspase-8 does not contribute to Akt phosphorylation. NB7 cells with the indicated caspase-8 constructs were serum-starved, stimulated with EGF, and assayed for Akt serine-473 phosphorylation by Western blotting. Densitometry of phospho-Akt and total Akt were used to derive ratios, which were plotted here; a lack of effect of caspase-8 on Akt phosphorylation was also observed with matrix-reattachment and serum re-addition (data not shown). 


\section{Figure 5}

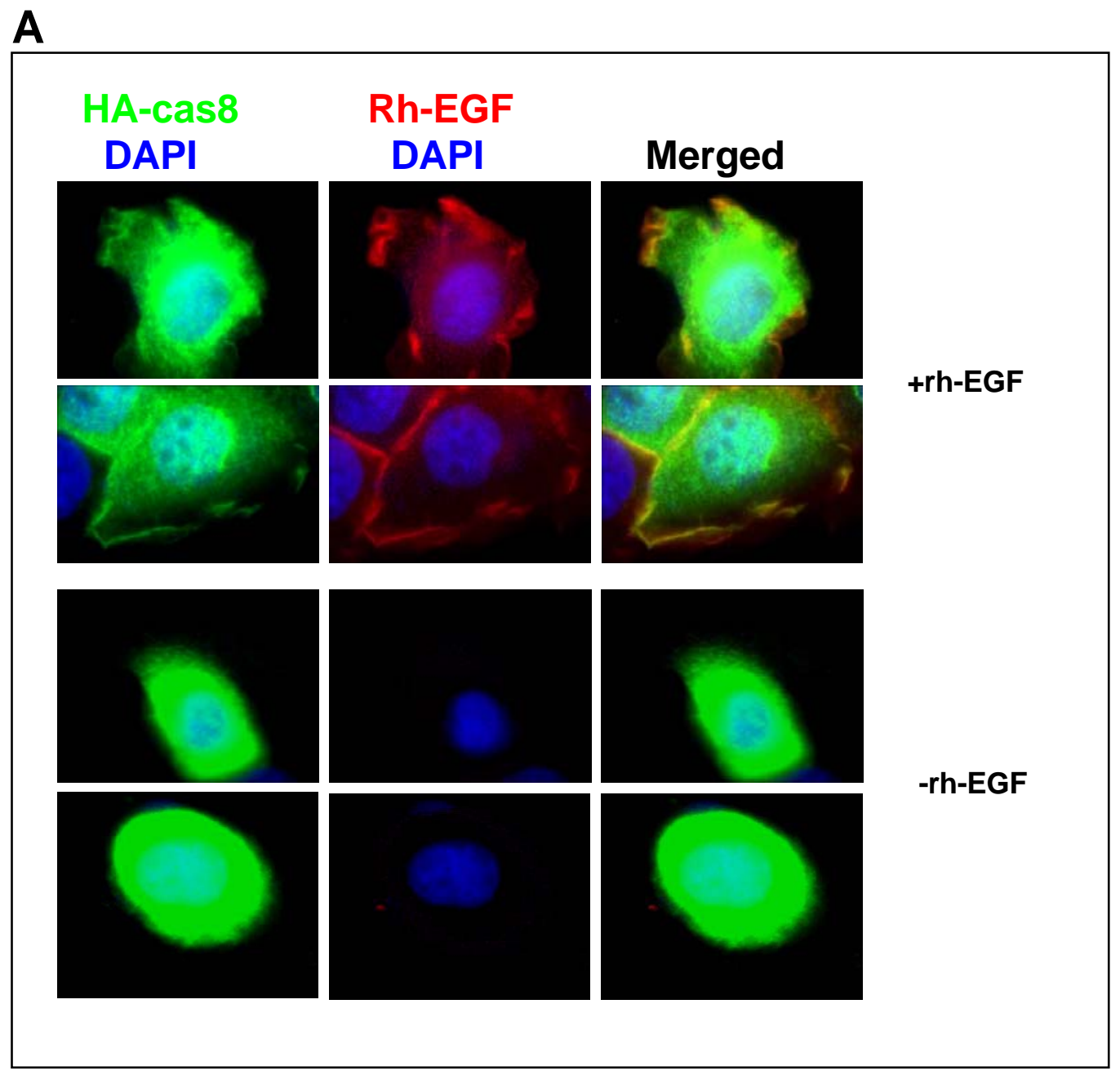

Figure 5: Caspase-8 relocalizes to cell periphery upon EGF treatment and colocalizes with rhodamine-labeled EGF (rh-EGF). A. Rh-EGF treatment colocalizes with HA-C360A caspase-8 in A431 cells 
B

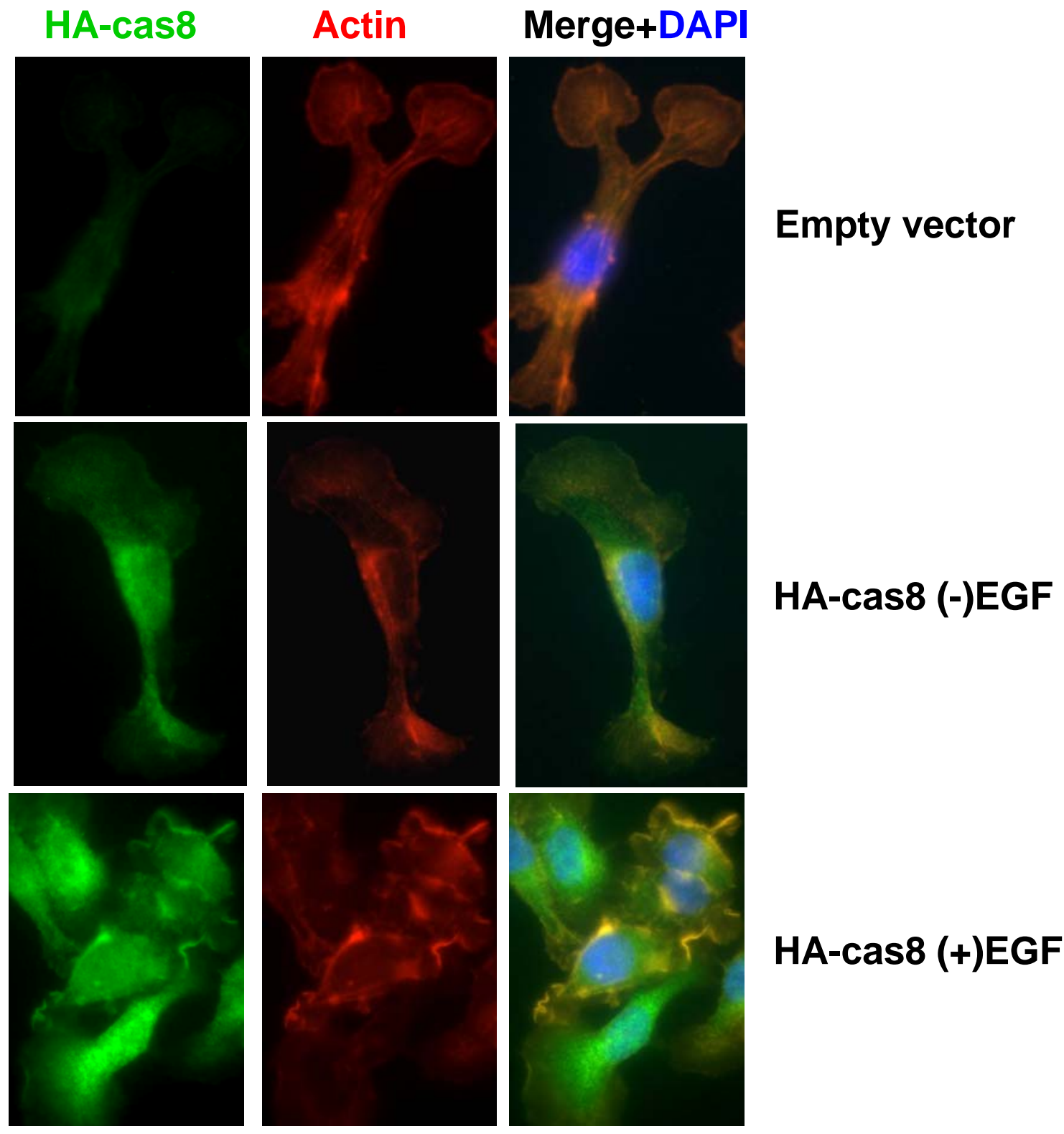

Figure 5: Caspase-8 relocalizes to cell periphery upon EGF treatment. B. HA-C360A caspase-8 localizes to the cell periphery upon EGF treatment. MTLNs were serum starved and treated with 100nM EGF for 5 minutes and IF done for HA and actin. 


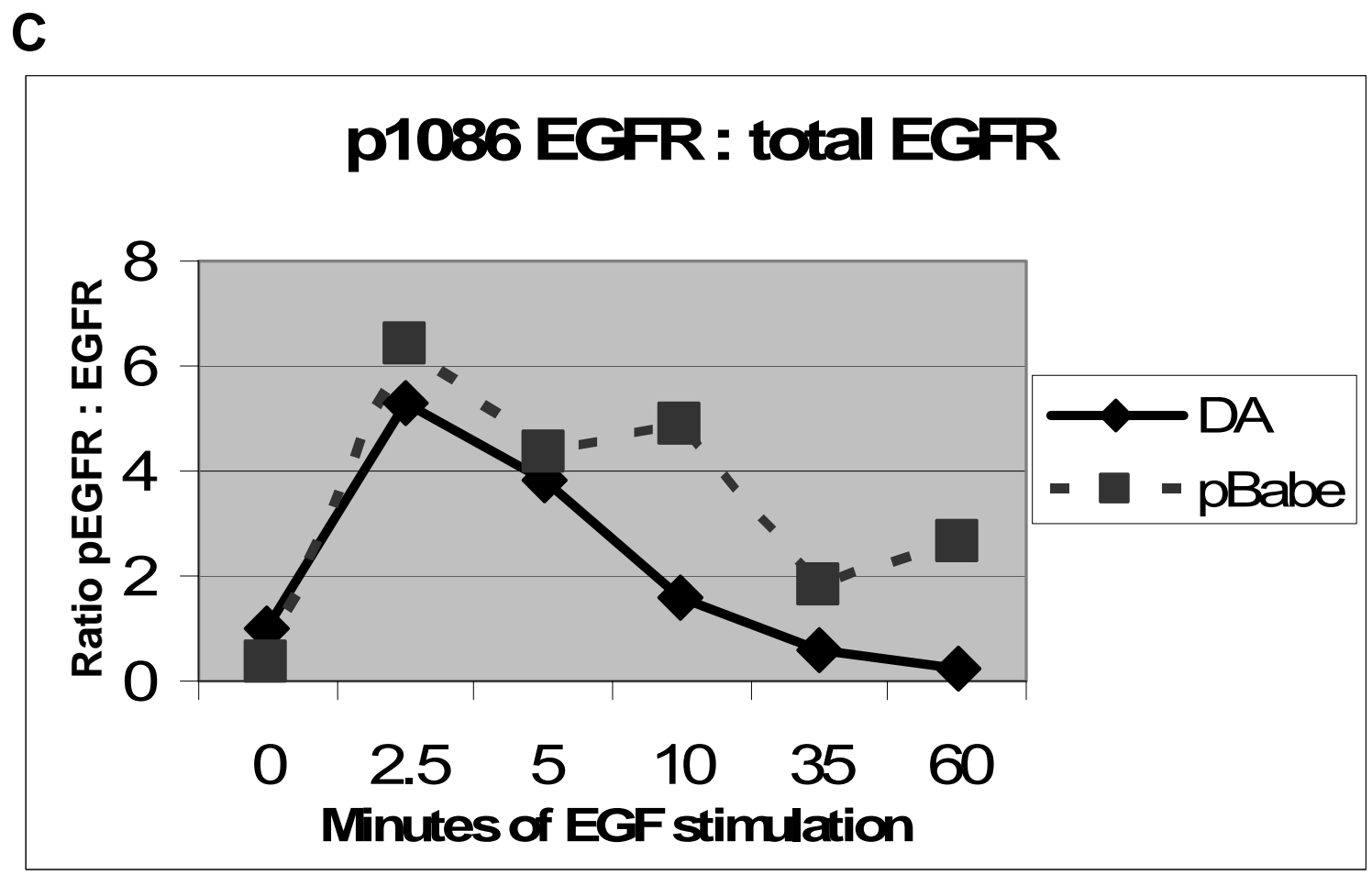

Figure 5: Caspase-8 relocalizes to cell periphery upon EGF treatment. C. Caspase-8 does not influence EGFR phosphorylation. 


\section{Figure 6}

A

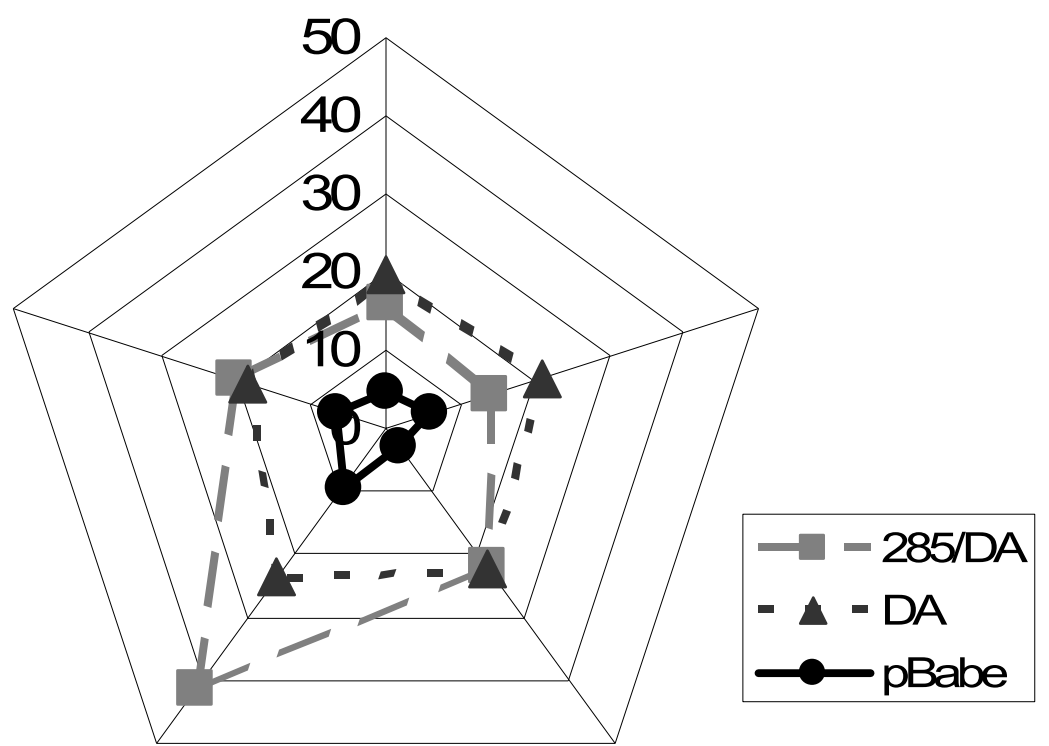

B

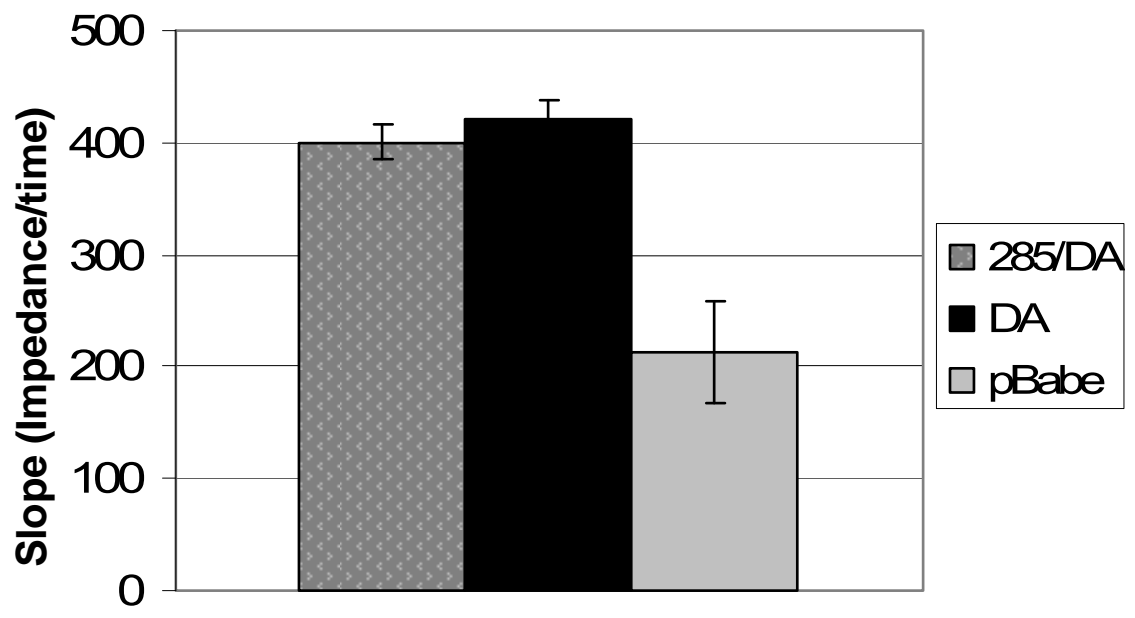

Figure 6. D285H, a naturally occurring SNP does not influence cellular motility or adhesion. Upper panel single cell random migration of NB7 with D285H, DA, or pBabe puro vector control expression. Lower panel - slope of ECIS adhesion assay. The slope represents cell adhesion and spreading across time. 


\section{Figure 7}

A

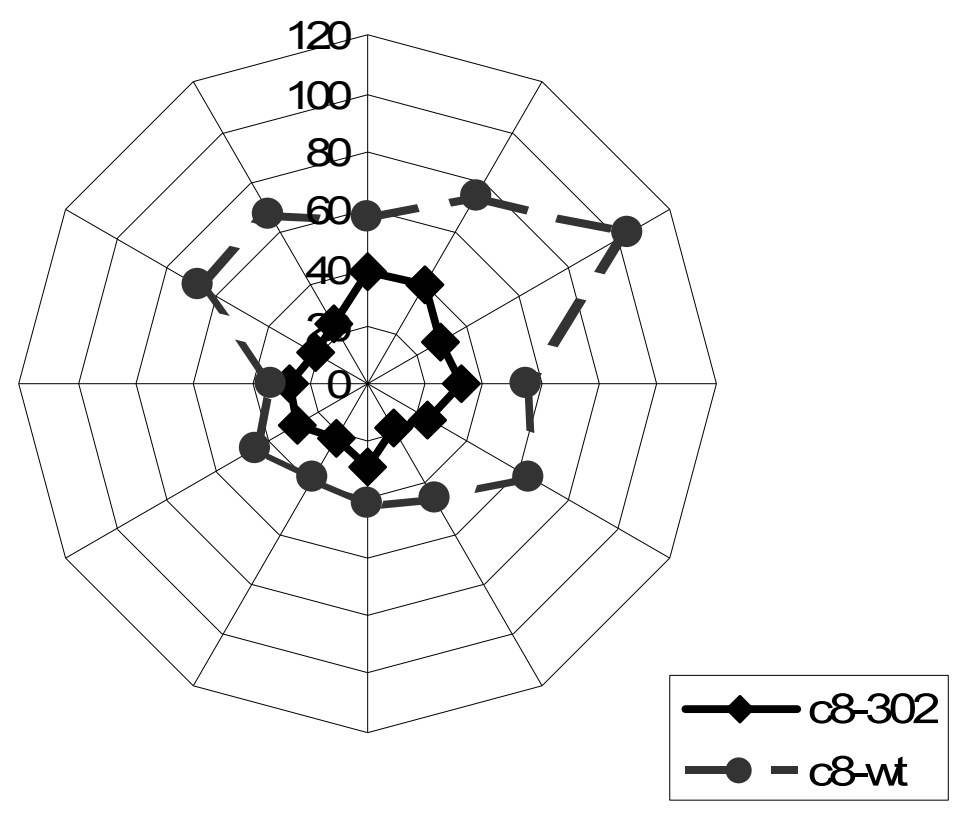

B

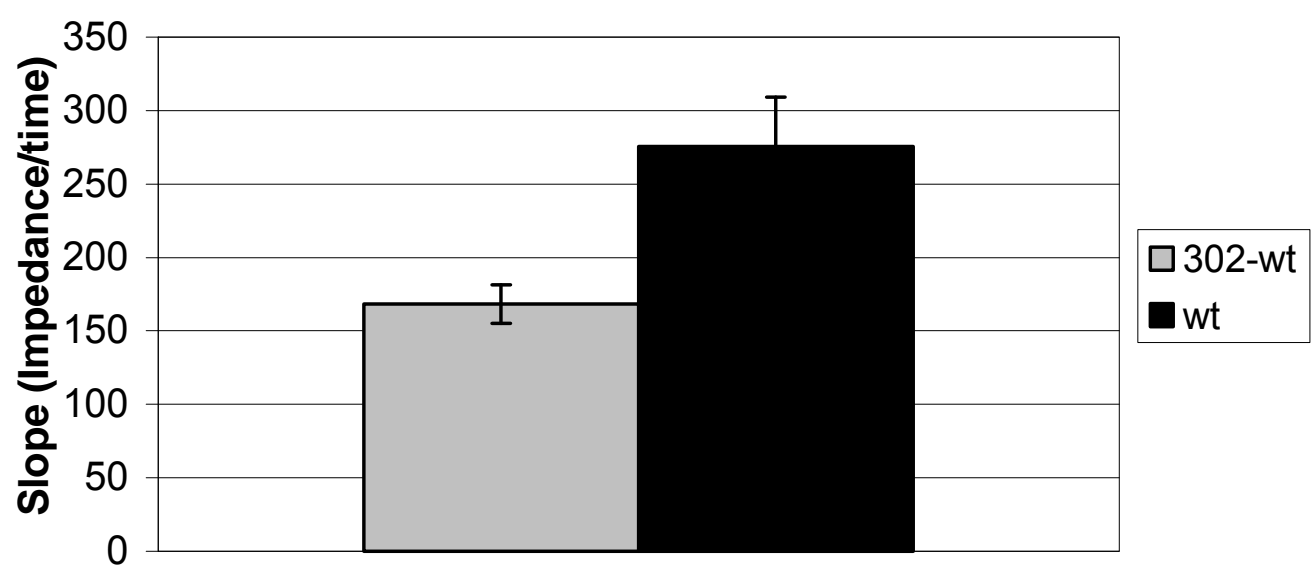

Figure 7. D302H, a mutation adjacent to a YXXM motif influences cellular motility and adhesion. Upper panel single cell random migration of $\mathrm{NB} 7$ with $\mathrm{D} 302 \mathrm{H}$, wt, or pBabe puro vector control expression. Wt caspase-8 expressing cells migrate better than $\mathrm{D} 302 \mathrm{H}$ cells. Lower panel - slope of ECIS adhesion assay. The slope represents cell adhesion and spreading across time. Wt cells attach and spread faster than $\mathrm{D} 302 \mathrm{H}$ expressing cells. 


\section{Figure 8}

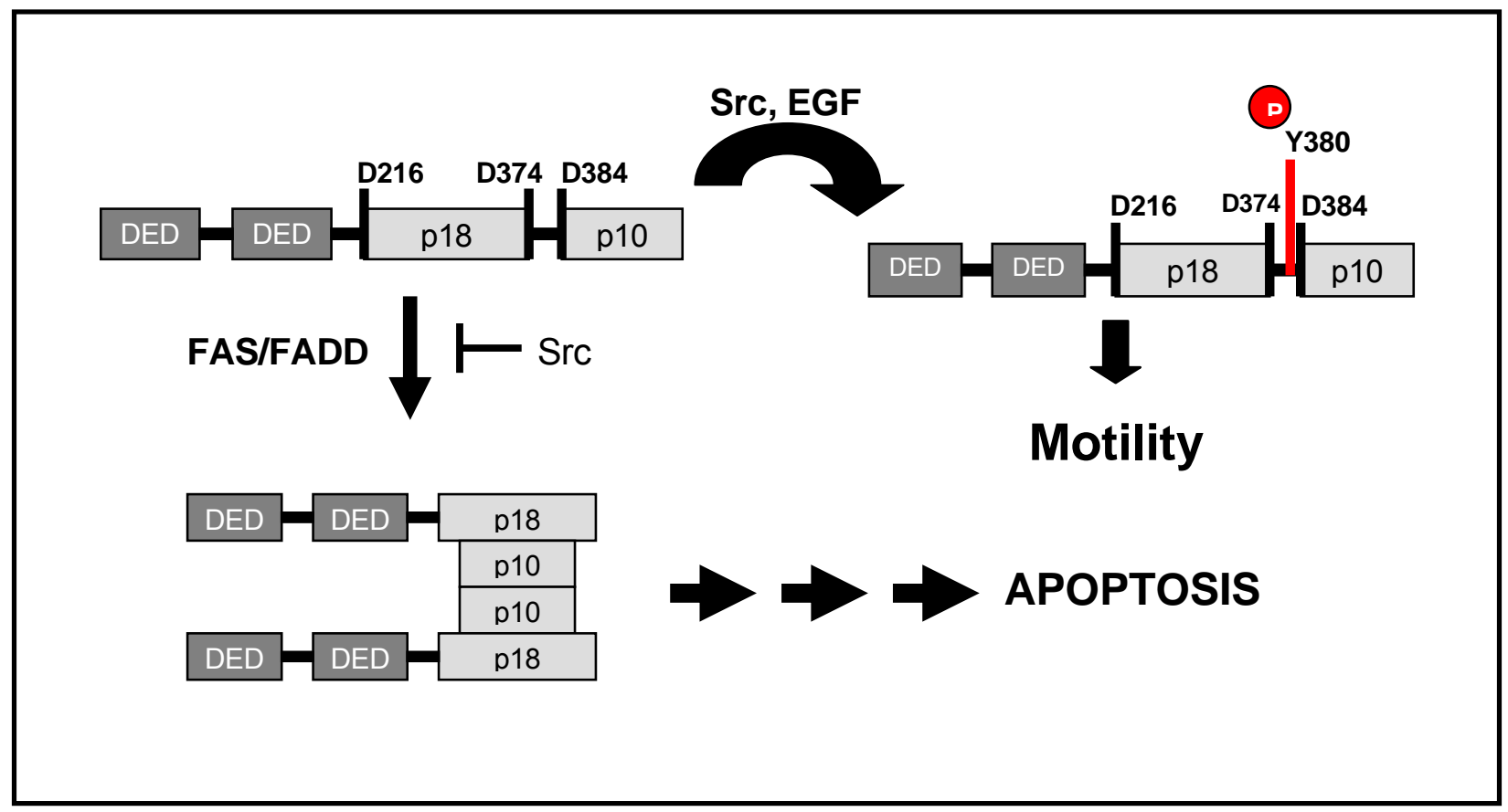

Figure 8: Caspase-8 is involved in a apoptosis motility switch. The phosphorylation of caspase- 8 by Src [5] blocks the first apoptotic autocleavage event of caspase-8's progression to apoptosis [6]. Phosphorylated caspase- 8 is able to promote interaction with the $p 85$ subunit of PI3K and promote cell migration. 


\section{References}

1. Helfer, B., et al., Caspase-8 promotes cell motility and calpain activity under nonapoptotic conditions. Cancer Res, 2006. 66(8): p. 4273-8.

2. Yamaguchi, H., J. Wyckoff, and J. Condeelis, Cell migration in tumors. Curr Opin Cell Biol, 2005.

3. Teitz, T., et al., Caspase 8 is deleted or silenced preferentially in childhood neuroblastomas with amplification of MYCN.[comment]. Nature Medicine, 2000. 6(5): p. 529-35.

4. Shivapurkar, N., et al., Differential inactivation of caspase-8 in lung cancers. Cancer Biol Ther, 2002. 1(1): p. 65-9.

5. Cursi, S., et al., Src kinase phosphorylates Caspase-8 on Tyr380: a novel mechanism of apoptosis suppression. Embo J, 2006. 25(9): p. 1895-905.

6. Chang, D.W., et al., Interdimer processing mechanism of procaspase-8 activation. Embo J, 2003. 22(16): p. 4132-42.

7. Luo, J. and L.C. Cantley, The negative regulation of phosphoinositide 3-kinase signaling by p85 and it's implication in cancer. Cell Cycle, 2005. 4(10): p. 130912.

8. Fukazawa, T., et al., Tyrosine phosphorylation of $\mathrm{Cbl}$ upon epidermal growth factor (EGF) stimulation and its association with EGF receptor and downstream signaling proteins. J Biol Chem, 1996. 271(24): p. 14554-9. 
9. Barfod, E.T., et al., Cloning and expression of a human CDC42 GTPaseactivating protein reveals a functional SH3-binding domain. J. Biol. Chem., 1993. 268(35): p. 26059-26062.

10. Sjolander, A. and E.G. Lapetina, Agonist-induced association of the p21ras GTPase-activating protein with phosphatidylinositol 3-kinase. Biochem Biophys Res Commun, 1992. 189(3): p. 1503-8.

11. Guinebault, C., et al., Integrin-dependent translocation of phosphoinositide 3kinase to the cytoskeleton of thrombin-activated platelets involves specific interactions of p85 alpha with actin filaments and focal adhesion kinase. J Cell Biol, 1995. 129(3): p. 831-42.

12. Gentili, C., S. Morelli, and A. Russo De Boland, Involvement of PI3-kinase and its association with c-Src in PTH-stimulated rat enterocytes. J Cell Biochem, 2002. 86(4): p. 773-83.

13. Hill, K.M., et al., N-terminal domains of the class ia phosphoinositide 3-kinase regulatory subunit play a role in cytoskeletal but not mitogenic signaling. J Biol Chem, 2001. 276(19): p. 16374-8.

14. Zheng, Y., S. Bagrodia, and R.A. Cerione, Activation of phosphoinositide 3kinase activity by Cdc42Hs binding to p85. J Biol Chem, 1994. 269(29): p. 18727-30.

15. Tolias, K.F., L.C. Cantley, and C.L. Carpenter, Rho family GTPases bind to phosphoinositide kinases. J Biol Chem, 1995. 270(30): p. 17656-9.

16. Boatright, K.M., et al., $A$ unified model for apical caspase activation. Molecular Cell, 2003. 11(2): p. 529-41. 
17. Vojtek, A.B., S.M. Hollenberg, and J.A. Cooper, Mammalian Ras interacts directly with the serine/threonine kinase Raf. Cell, 1993. 74(1): p. 205-14.

18. Senft, J., B. Helfer, and S.M. Frisch, Caspase-8 interacts with the $p 85$ subunit of phosphatidylinositol 3-kinase to regulate cell adhesion and motility. Cancer Res, 2007. 67(24): p. 11505-9.

19. MacPherson, G., et al., Association of a common variant of the CASP8 gene with reduced risk of breast cancer. J Natl Cancer Inst, 2004. 96(24): p. 1866-9.

20. Cox, A., et al., A common coding variant in CASP8 is associated with breast cancer risk. Nat Genet, 2007. 39(3): p. 352-8.

21. Sun, T., et al., A six-nucleotide insertion-deletion polymorphism in the CASP8 promoter is associated with susceptibility to multiple cancers. Nat Genet, 2007. 39(5): p. 605-13.

22. Barbero, S., et al., Identification of a critical tyrosine residue in caspase 8 that promotes cell migration. J Biol Chem, 2008.

23. Finlay, D. and K. Vuori, Novel noncatalytic role for caspase-8 in promoting SRC-mediated adhesion and Erk signaling in neuroblastoma cells. Cancer Res, 2007. 67(24): p. 11704-11.

24. Siegel, R.M., et al., Death-effector filaments: novel cytoplasmic structures that recruit caspases and trigger apoptosis. J Cell Biol, 1998. 141(5): p. 1243-53.

25. Ainhoa Mielgo, V.T., Michael Schmid, Simone Barbero, David Shields, Sam Zeitlin, Dwayne Stupack. The death effector domains of caspase 8 induce senescence [abstract]. in Proceedings of the 99th Annual Meeting of the 
American Association for Cancer Research. 2008 Apr 12-16; San Diego, CA. Philadelphia (PA): AACR; 2008. Abstract nr 1837.San Diego, CA.

26. Innocenti, M., et al., Phosphoinositide 3-kinase activates Rac by entering in a complex with Eps8, Abi1, and Sos-1. J Cell Biol, 2003. 160(1): p. 17-23.

27. Jang, J.Y., et al., Over-expression of Adenine Nucleotide Translocase 1 (ANT1) induces apoptosis and tumor regression in vivo. BMC Cancer, 2008. 8(1): p. 160.

28. Le Bras, M., et al., Chemosensitization by knockdown of adenine nucleotide translocase-2. Cancer Res, 2006. 66(18): p. 9143-52.

29. Rush, J., et al., Immunoaffinity profiling of tyrosine phosphorylation in cancer cells. Nat Biotechnol, 2005. 23(1): p. 94-101.

30. Brachmann, S.M., et al., Role of phosphoinositide 3-kinase regulatory isoforms in development and actin rearrangement. Mol Cell Biol, 2005. 25(7): p. 2593606.

31. Fukata, M., M. Nakagawa, and K. Kaibuchi, Roles of Rho-family GTPases in cell polarisation and directional migration. Curr Opin Cell Biol, 2003. 15(5): p. 590-7.

32. Jimenez, C., et al., Role of the PI3K regulatory subunit in the control of actin organization and cell migration. J Cell Biol, 2000. 151(2): p. 249-62. 


\section{Chapter 4}

\section{Caspase-2 promotes cellular adhesion, FAK activation, and the breakdown of actin stress fibers}




\section{Abstract}

Caspase 2 plays important roles in DNA damage-induced, detachment-induced, and heat shock-induced apoptosis. Additional biologic functions are likely; while the developmental phenotype of caspase 2 knockout mice is normal, it is often the case that the same gene knockout examined in different mouse strains has varying effects. Here, we demonstrate that caspase 2 has additional roles in the cell beyond apoptosis. In this study we demonstrate that caspase 2 promotes cell-matrix adhesion focal contact formation and FAK phosphorylation. Caspase 2 also influences stress fiber integrity in response to protein kinase-C activation. Insights into the mechanism whereby caspase 2 influences cytoskeletal processes with emphasis on Rho/ROCK/LIMK/cofilin and Rho/ROCK/MLC pathways are discussed herein. 


\section{Introduction}

Potential non-apoptotic roles for caspase 2 stem from studies of DRONC. DRONC, the Drosophila homolog of caspases 2 and 9, is another caspase with a non-apoptotic function. DRONC and its inhibitor, DIAP1 (Drosophila inhibitor of apoptosis 1) are connected to border cell migration during Drosophila development though interaction of DIAP1 with the Rac pathway [1]. Regulation of this function is through DmIKKE, an antagonist of DIAP [2, 3] that suppresses actin polymerization [4]; other factors may be involved.

Non-apoptotic roles are emerging for caspase 2 as well. Caspase 2 is involved in the SREBP mediated increase of lipid levels in that the siRNA knock down of caspase 2 actually inhibits lipid amplification [5]. Interestingly, cholesterol-lowering drugs, statins (e.g. lovastatin), increase caspase 2 gene expression [5] and alter the actin cytoskeleton through caspase 2 [6]. This process may be mediated through a caspase-2-RhoB interaction [7].

Caspases are also associated with Rho kinase (ROCK) in both apoptotic and nonapoptotic contexts. ROCK I cleavage by caspases is associated with apoptotic membrane blebbing [8]. ROCK II is also associated with myosin light chain (MLC) contractility and membrane blebbing [9]. ROCKII is also associated with a potentially nonapoptotic function of caspase 2. Thrombin-induced ROCK activation and ROCKII cleavage has been proposed to be mediated by caspase-2 [10]. 
Taken together, caspase 2 control of cytoskeletal contractility and, in turn cell adhesion and stress fiber dynamics, though the RhoA/ROCK/MLC and/or RhoA/ROCK/LIMK/cofilin/actin pathway is not unlikely [11-14]. This chapter will examine the relationship of caspase-2 with cellular adhesion and stress fiber dynamics and address possible mechanisms. 


\section{Experimental Procedures}

Cell lines: The caspase 2 deficient and wild type MEF cells (C2-/- and C2+/+ respectively) were provided by $\operatorname{Dr}$ Andreas Strasser [15]. These were immortalized by infection with an SV40 T-antigen retroviral construct. To generate rheoswitchinducible caspase- 2 cell lines, a mouse caspase- 2 expressing derivative of the rheoswitch vector pNEBR-X1 was constructed by subcloning wild type caspase-2 (provided by Sharad Kumar) into the pNEBRX1 vector. The caspase-2-X1 construct, pBabe puromycin construct and inducing vector pNEBR-R1 were linearized at a single cut site in a non-coding portion of the vector. Co-electroporation was done using the Rheoswitch vectors and puromycin as a drug selection marker. Electroporation is preformed using the Amaxa Biotechnology Nucleofector II. Clones of each cell construct were selected with $1.5 \mu \mathrm{g} / \mathrm{mL}$ puromycin (Calbiochem), isolated, and screened for induction.

Adhesion assays: Electrical Cell-Substratum Impedance Sensing (ECIS). Cells were counted $\left(5 \times 10^{\wedge} 5\right.$ cells/well) and let attach to the chambers $\left(\sim 1 \mathrm{~cm}^{2}\right)$ of a tenelectrode ECIS array (Applied Biophysics, Troy, New York; www.biophysics.com) that were pre-coated with fibronectin. Adhesion was done in DMEM in the absence of serum and was observed until a steady impedance value was reached. As the cells attached to the electrodes of the ECIS array, the impedance increased. The impedance was monitored at $40 \mathrm{kHz}$ frequency and was carried out in triplicate chambers. Data were exported to Excel for calculation of slopes. 
Adhesion analysis by Immunofluorescence: Cells are prepared similarly as above except that the cells are serum starved over night in 0.5\% FBS containing DMEM. Cells are then attached to $15 \mu \mathrm{g} / \mathrm{mL}$ fibronectin coated cover slips and are fixed using 4\% paraformaldehyde in PBS. Immunofuorescent preparation/staining is then done (see below).

Adhesion for protein activation: Cells serum starved over night in $0.5 \%$ FBS containing DMEM and let attach to $15 \mathrm{ug} / \mathrm{mL}$ fibronectin coated wells. Wells were covered with matrix over night and then remaining well surfaces were blocked in $3 \%$ BSA containing DMEM for 2 hours. Cells are then detached from wells using TrypLE Express (Invitrogen) and resuspended in $0.5 \%$ FBS, 3\%BSA containing DMEM. Cells are then allowed to attach for the designated time course and lysed with $5 \% \beta$ Mercaptoethanol containing laemmli sample buffer. Western blots of lysates were then performed using primary antibodies: cofilin, phospho-cofilin (Cell Signaling), myosin light chain (from R. Wysolmerski), phospho-myosin light chain (Cell Signaling), focal adhesion kinase, phospho-focal adhesion kinase (Y397, Sigma) or RhoA (Santa Cruz Biotechntology) followed by species specific secondary HRP conjugated secondary antibodies (Zymax).

PMA treatment: Cells were grown to equal but sub-confluent densities and serum starved for at least 8 hours before $0.1 \mu \mathrm{M}$ PMA treatment. The PMA was prepared in warm serum free media, which replaced the serum starving media on the cells for the 
PMA treatment time course. Cells were then lysed for western blot analysis. If the samples were for immunofluorescence (IF), the cells were initially attached to glass cover slips and then treated similarly through PMA treatment. The cells were then fixed with paraformaldehyde and prepared for IF.

Immunofluorescence: Cells that were fixed to cover slips were permeabilized with 0.1\% Triton X-100. $4 \mathrm{G} 10$ phosphotyrosine (Upstate) or vinculin (Sigma) primary antibodies were used and Alexa-488 labeled secondary antibody (Pierce) was applied. For actin staining, rhodamine phalloidin was applied with the secondary antibody. Cover slips were then mounted with Vectashield mounting media with DAPI (Vector Labs). Slide analysis was performed using a Zeiss Axiovert 200M microscope with associated AxioVision software (Carl Zeiss, Göttingen Germany) for image analysis. 


\section{Results}

Caspase 2 promotes cellular adhesion. Cellular adhesion was compared by ECIS using RSL1-induced vs uninduced caspase-2-rheoswitch cell lines, in which induced expression was verified by western blotting (figure 1A). Caspase 2 expression promoted cellular adhesion to matrigel (BD Biosciences, Figure 1B, 1C) and fibronectin (Figure 1D). Similar results were obtained comparing caspase $2 \mathrm{wt}$ and knock out MEFs (data not shown).

To further characterize caspase-2 effects on cellular adhesion, the kinetics of focal contact formation were also examined. At 16 minutes of adhesion, focal complex formation was increased in caspase 2 expressing cells above that in the uninduced cells (Figure 2). Both vinculin and phosphotyrosine staining yield similar results. Staining patterns at 26 minutes of adhesion were consistant with the trends witnessed at 16 minutes but were not as striking (data not shown).

Caspase-2 promotes focal adhesion kinase activation upon adhesion. A time course of adhesion, comparing the uninduced vs induced caspase-2-Rheoswitch cells, also yields a difference in the rate of focal adhesion kinase (FAK) phosphorylation on tyrosine 397 (Figure 3). Similar results were obtained comparing caspase 2 wt MEF and caspase 2 knock out cells (unpublished data SMF). Caspase 2 wt MEFs are shown in Figure 3 for comparison to the induced caspase-2Rheoswitch cells. 
These results suggest that caspase-2 has an important role in mediating cellular adhesion. Caspase-2 may be influencing FAK after engagement of integrins directly. Due to the manner in which the FAK activation assays were conducted, it is not known if FAK is being directly affected or whether caspase-2 is influencing another cytoskeletal component to mediate FAK activation.

Caspase 2 influences stress fiber integrity. Using phorbol esters to mimic DAG signaling in cells, PKC becomes activated. This activation leads to Src activity and cytoskeletal reorganization, such as the breakdown of stress fibers [16, 17]. To determine whether caspase 2 affected this pathway a comparison of phorbol 12 myristate 13-acetate (PMA) treatment of caspase 2 inducible MEF was performed (Figure 4). Surprisingly, caspase 2 null cells were non-responsive to the stress fiber degrading effects of PMA. This finding is novel because it shows caspase 2 mediating stress fiber breakdown in the absence of an apoptotic stimuli. Similar results were found in MEFs using a vdVAD capsase-2 inhibitor (Figure 4C). Interestingly, the inhibition of caspase-8 by IETD was similar to wild type MEF suggesting that regulation of stress fiber dynamics is unique to the initiator caspase- 2 .

How does caspase-2 alter the cytoskeleton? Caspase 2 has been linked to several proteins that alter the cytoskeleton (ROCK, Rho, spectrin etc). Rho/ROCK have a strong association with focal contact formation (reviewed in [18]) and ROCK cleavage is shown to be mediated by caspase $2[10]$. Interestingly, procaspase-2 can 
be catalytically active [19], perhaps allowing it to cleave ROCK in this non-apoptotic context.

To check the ability of caspase 2 to cleave ROCK and look at overall ROCK activity in our system we assesed ROCKII cleavage in the RheoSwitch inducible caspase 2 cells (Figure 5). We stimulated serum starved cells with thrombin and performed a western blot on their lysates. ROCK activity was also analyzed in the context of cellular adhesion. Since we saw a striking difference in FAK phosphorylation (Figure 3), we used a similar time line to detect ROCK cleavage. Though preliminary, in our hands, caspase-2 promoted thrombin stimulated ROCK cleavage (Figure 5) because there is a decrease in total ROCK protein (although there was no evidence of ROCK cleavage products, perhaps due to limitations of the antibody or sensitivity of this western blot). Note also that the equal loading of this western requires confirmation with internal loading controls, and that the ROCKII protein migrated as a larger protein $(\sim 180 \mathrm{kDa})$ than would be predicted from its molecular weight (160kDa), although the same size band was observed using two different antibodies (data not shown).

RhoA activation was also analyzed. Adhesion is influenced upstream of ROCK by RhoA. During initial adhesion, RhoA activity decreases transiently then increases (reviewed in [18]). RhoA experiments seeking activation of RhoA upon cellular adhesion were initially unsuccessful. We were unable to detect an effect of caspase-2 upon RhoA activation following LPA stimulation (YJ Cho, unpublished data). When 
combined, the results suggest that the effect of caspase- 2 may be downstream of RhoA, at the level of ROCKII.

Cofilin and MLC phopshorylation are downstream of ROCK. ROCK, when active, activates LIMK which then phosphorylates cofilin rendering it inactive [14]. We predicted that since caspase 2 null cells do not break down stress fibers as readily upon PMA treatment that they would not inactivate ROCK, thus not alter the phosphorylation of cofilin. Neither cellular adhesion nor PMA treatment altered the levels of phosphorylated cofilin between samples with and without caspase-2 (Figure 6). These results suggest that cofilin is not altered, nor is the LIMK/cofilin pathway being affected due to unaltered cofilin phosphorylation status as a readout.

ROCK activity is noted to increase the phosphorylation of MLC and promote maintenance focal adhesions and stress fibers under normal conditions [11]. LPA stimulation did not alter MLC phosphorylation in the context of caspase-2 expression (unpublished data YJ Cho). The affect of adhesion with caspase-2 upon MLC phosphorylation has yet to be determined. Caspase-2 did not influence phosphorylation of myosin light chain upon PMA treatment (Figure 7). 


\section{Discussion and Future Studies}

Caspase-2 influences cytoskeletal processes through the promotion of cellular adhesion and stress fiber breakdown. Present, developing literatures on caspase-2 propose its connection to Rho/ROCK signaling pathways $[7,10]$. In the context of cellular adhesion or stress fiber breakdown, caspase-2 did not alter Rho/ROCK/MLC or Rho/ROCK/LIMK/cofilin pathways. While the pathway studies are preliminary they do not suggest that caspase-2 was required for their proper activation/execution.

The future of this project involves elucidation of the pathway in which caspase- 2 is influencing cytoskeletal dynamics. Caspase-2 catalytic activity may be required, as seen in Sapet et al [10], or it may be involved independent of catalytic activity as was seen with caspase-8 (Chapter 3). Proteomic screens using a dual tagged (FLAG and S-tag) caspase-2, similar to what is being done with caspase-8, will be done to identify potential interaction partners. Caspase-2 may influence cellular adhesion and stress fiber dynamics via separate mechanisms. The inhibition of caspase-2 was able to mimic caspase-2 knock outs in the maintenance of stress fibers upon DAG like signaling. This suggests that caspase-2's catalytic activity may be required for stress fiber dynamics.

Caspase-2 knock out MEFs also have an accumulation of multinucleate cells in comparison to wild type MEFs (B Helfer and $\mathrm{YJ}$ Cho, data not shown). This accumulation of multinucleate cells could be due to aberrant mitosis or cytokinesis. Collaboration and work being continued by YoungJin Cho Cunnick has established a 
potential connection of caspase-2 in the regulation of cell cycle progression. Caspase-2 expressing cells synchronized by nocodazole and then allowed to go on though the cell cycle exhibit an increased activation of CDC42 above caspase-2 knock out MEFs. Furthermore, western blots of caspase-2 from these experiments display two bands (a doublet) suggesting a potential caspase-2 modification, such as phosphorylation (data not shown).

According to the hypothesis that caspase-2 influences ROCK cleavage, caspase-2's catalytic activity would be required for its downstream cytoskeletal effects. The data using the caspase-2 specific inhibitor zVDVAD-fmk, suggest that the catalytic activity of caspase-2 is required for the breakdown of stress fibers. It is possible that these two phenomena are controlled by caspase- 2 by both different mechanisms and forms of caspase-2. Another hypothesis was also considered; Caspase 2 complexes with RhoB to alter cellular response to PKC activation. RhoB has $85 \%$ sequence homology to both RhoA and RhoC [20]. RhoB is associated with cell survival but has been shown to induce stress fibers when over expressed (reviewed [20]). In studies examining the role of lovastatin in cells, the activation of Rho proteins is linked to the cytoskeletal changes brought upon by lovastatin treatment [21]. Furthermore, with caspase 2 , RhoB is shown to be in a complex with caspase 2 upon lovastatin treatment [7]. It is possible that caspase 2's association with RhoB is involved in PKC mediated rearrangement of stress fibers. We tested this hypothesis but were unable to distinguish heavy-chain antibody bands from caspase-2 detection to confirm proper IP, thus caspase-2 may interact with RhoB to influence cytoskeletal dynamics. Should 
caspase-2 interact with RhoB [7], its response may be via this interaction, suggesting a noncatalytic mechanism.

The recent connection of caspase-2 in cytoskeletal damage induced apoptosis [22] is of great relevance to our studies. The results of this study further support our connection of caspase-2 to cytoskeletal dynamics but also introduces additional means for which caspase-2 can be bringing about its influence. It is possible that a lack of caspase-2 expression, which allows abnormal cytoskeletal activities to occur, does so by preventing cell death, which would normally occur in caspase-2 expressing cells. The lack of apoptosis induction in the caspase- 2 null cells would therefore be allowing these abnormalities to manifest. Should caspase-2 expression exist solely for induction of apoptosis, tumors would be predicted to decrease its expression. Reasons for both its apoptotic and non-apoptotic reduction exist in gastric cancer and in leukemia. In the case of leukemia, lack of cellular adhesion would not be a reason to decrease caspase-2 expression, evasion of apoptosis such as DNA damage induced apoptosis would be beneficial. Accordingly, decreased caspase-2 expression has been linked to drug resistance in childhood acute lymphoblastic leukemia [23]. Loss of caspase-2 in gastric cancer [31] is understandable in terms of decreased anoikis. Since cells that are shed from the intestinal lining normally undergo anoikis [24], evasion of apoptosis induction along with a decreased adhesion would be beneficial to cancer in this context. 
Similar proteomic studies are going to be performed with caspase-2 to determine unique protein interactions that may influence cytoskeletal dynamics, cell cycle progression, or apoptosis induction via the cytoskeletal disruption mechanism discussed above. Caspase-2 protein interactions will be able to distinguish between non-apoptotic and apoptotic roles, aid in RhoB and CDC42 hypotheses, and help determine whether it is via caspase-2's catalytic activity it is producing its effects. .

A prediction for why caspase- 2 knock out MEFs are not embryonic lethal, is that MEF still have expression of caspase- 9 , which due to its structural similarity may be able to compensate for the loss of caspase-2 $[25,26]$. Humans with a caspase-8 inactivating mutation exhibit T-cell immunodeficiency, but are viable [27]. Capase-10 is credited in part for this phenomenon, in that it is structurally similar to caspase-8 and believed to be partially redundant $[26,28-30]$. It will be interesting to see what, if any nonapoptotic roles exist for caspase- 9 and if any of them coincide with those exhibited with caspase-2.

The significance of this project is evident in caspase 2's effects on cytoskeletal processes. While few cancers are noted to decease the presence of caspase 2 [23, 31] a tumor screen (Genotech) showed that others maintained if not increased expression of caspase-2 (Figure 8); this project provides information to why it would be beneficial for cells to alter caspase-2 expression. A decrease in cellular adhesion and altered cytoskeletal processes could have several cellular ramifications, such as the generation of multinucleate cells through unsuccessful cytokinesis [32, 33]. Future 
continuation of this project has the potential to develop into insights on caspase-2's influence over the cytoskeleton both under non- and apoptotic circumstances. Additionally, this project will be able to provide insight into determining if caspase-2 expression will alter patient survival in addition to apoptosis. 


\section{Figure 1}
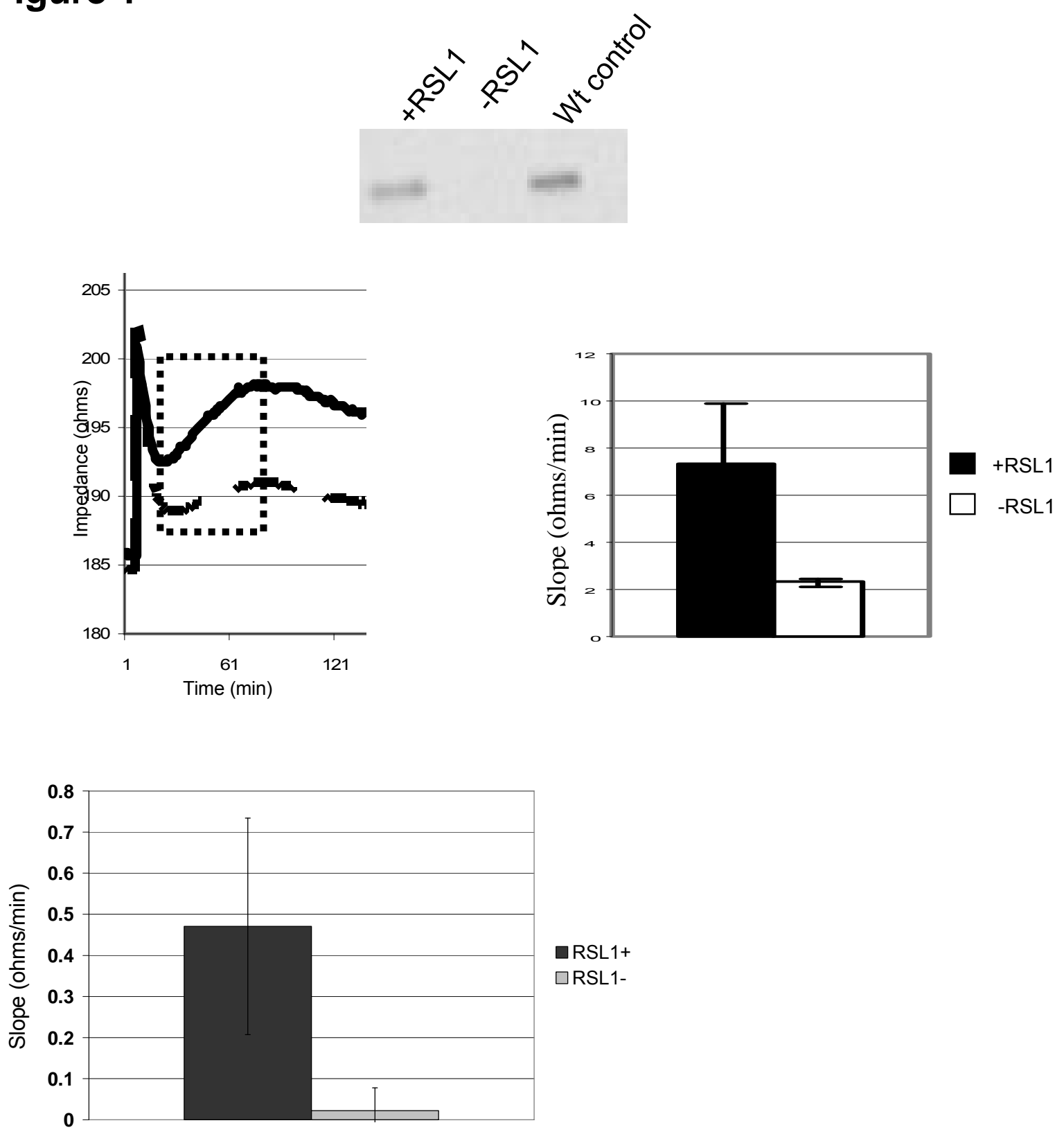

$\square \mathrm{RSL} 1+$

$\square$ RSL1-

Figure 1: Caspase-2 promotes cellular adhesion. A) Verification of caspase-2 induction. Cells are grown to confluence $+/$ - inducer and then lysed. B) ECIS plot of induced caspase-2 (expressing) cells (solid line) and uninduced cells (dashed line). The two lines represent the averages of each of three wells ( $X$ axis time, $Y$ impedance). As cells attach to the plates electrodes the impedance values increase. Panel $B$ is adhesion to Matrigel (BD Biosciences). C) Excel plot of the slope within the dashed box in panel B. Caspase-2 expressing cells attach faster than those lacking caspase-2. D) Excel plot of the slope of cells attaching in ECIS to fibronectin (Calbiochem). 


\section{Figure 2}
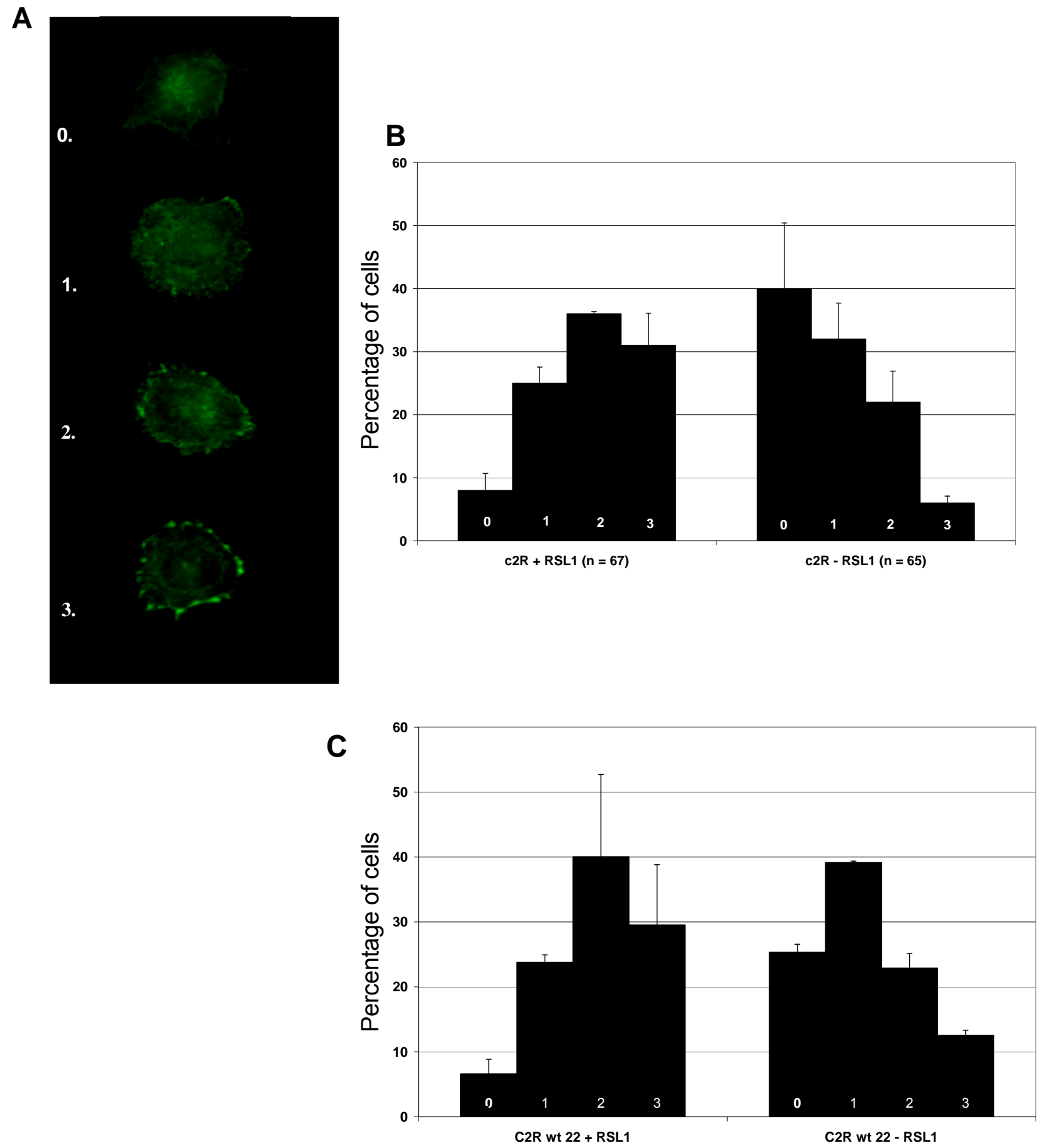

Figure 2: Caspase-2 promotes focal contact formation. A) Grading scale for degree of focal contact formation. $0=$ no contacts $1=$ few, faint contacts $2=$ contacts forming around the cell $3=$ bright mature contacts surrounding the cell. B) Graph of the degree of focal contact formation to fibronectin at 16 minutes of adhesion determined by vinculin staining. C) Degree of focal contact formation based upon phospho-tyrosine staining. 


\section{Figure 3}
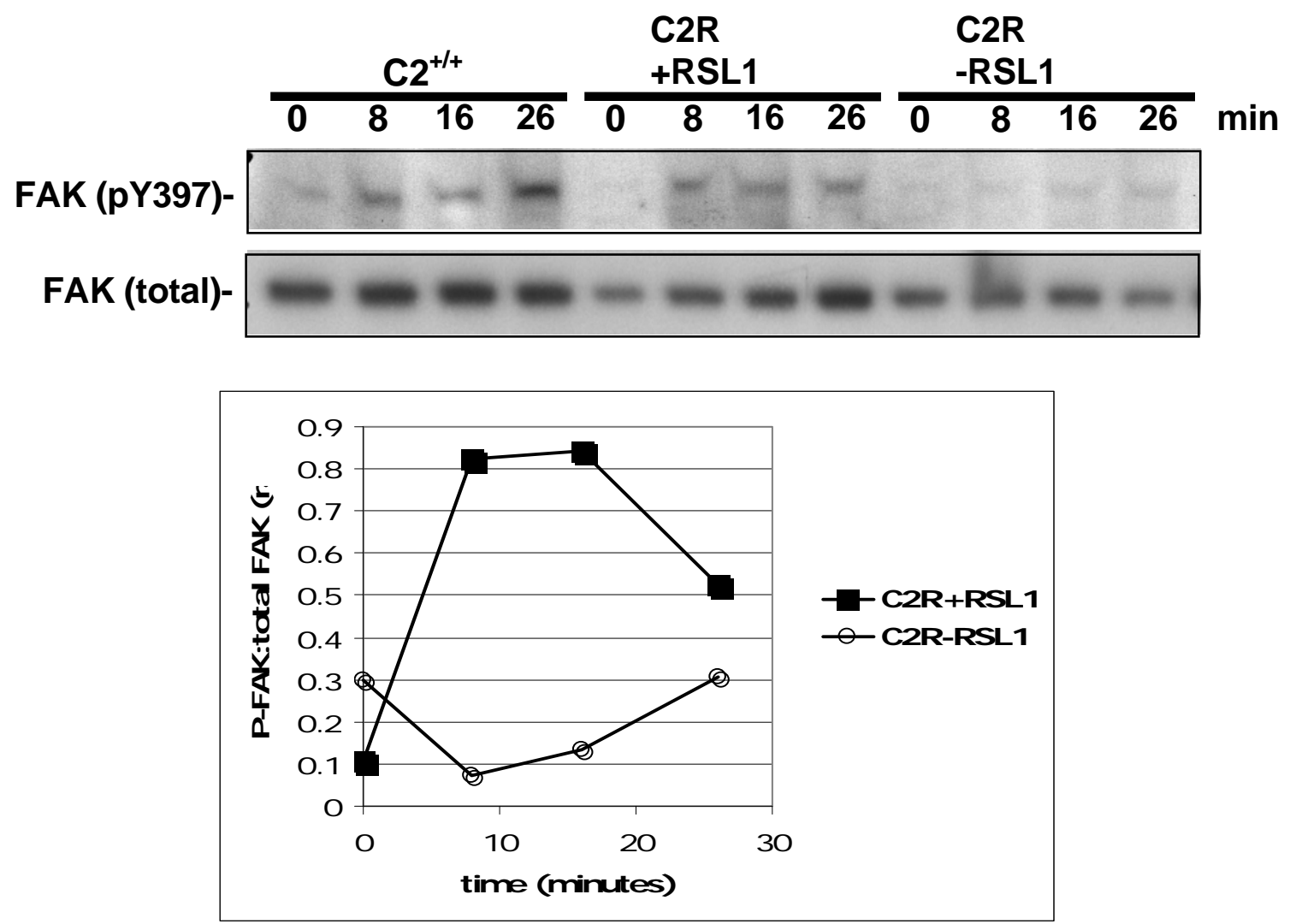

Figure 3: Caspase-2 promotes FAK phosphorylation at Y397 upon cellular adhesion. Caspase-2 wt and induced caspase-2 expressing cells showed pFAK levels above the uninduced, non-caspase-2 expressing cells. Upper panel is of the FAK western blot and densitometry plot of the ratio pFAK to total FAK levels is below. 


\section{Figure 4}

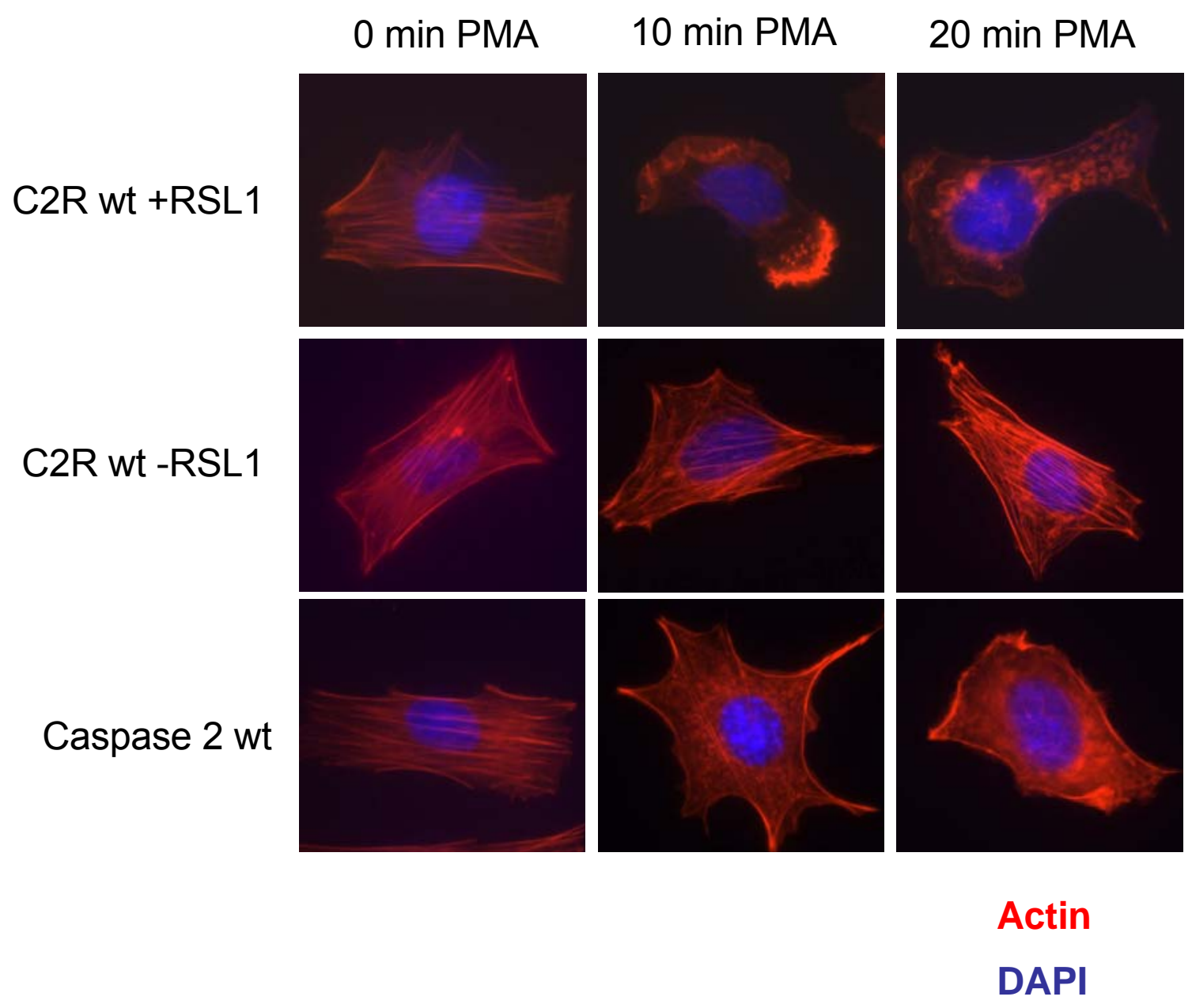

Figure 4: Caspase-2 promotes the loss of stress fibers upon PMA/TPA treatment. Wild type caspase-2 inducible cells (C2Rwt) both induced (+RSL1) and uninduced (-RSL1) in comparison with wild type MEF (caspase-2 wt) were untreated (time 0 ) and treated with PMA for 10, 20 minutes. Caspase-2 uninduced cells did not break down stress fibers where as the wild type and induced cell lines did show breakdown of stress fibers (top). 
B

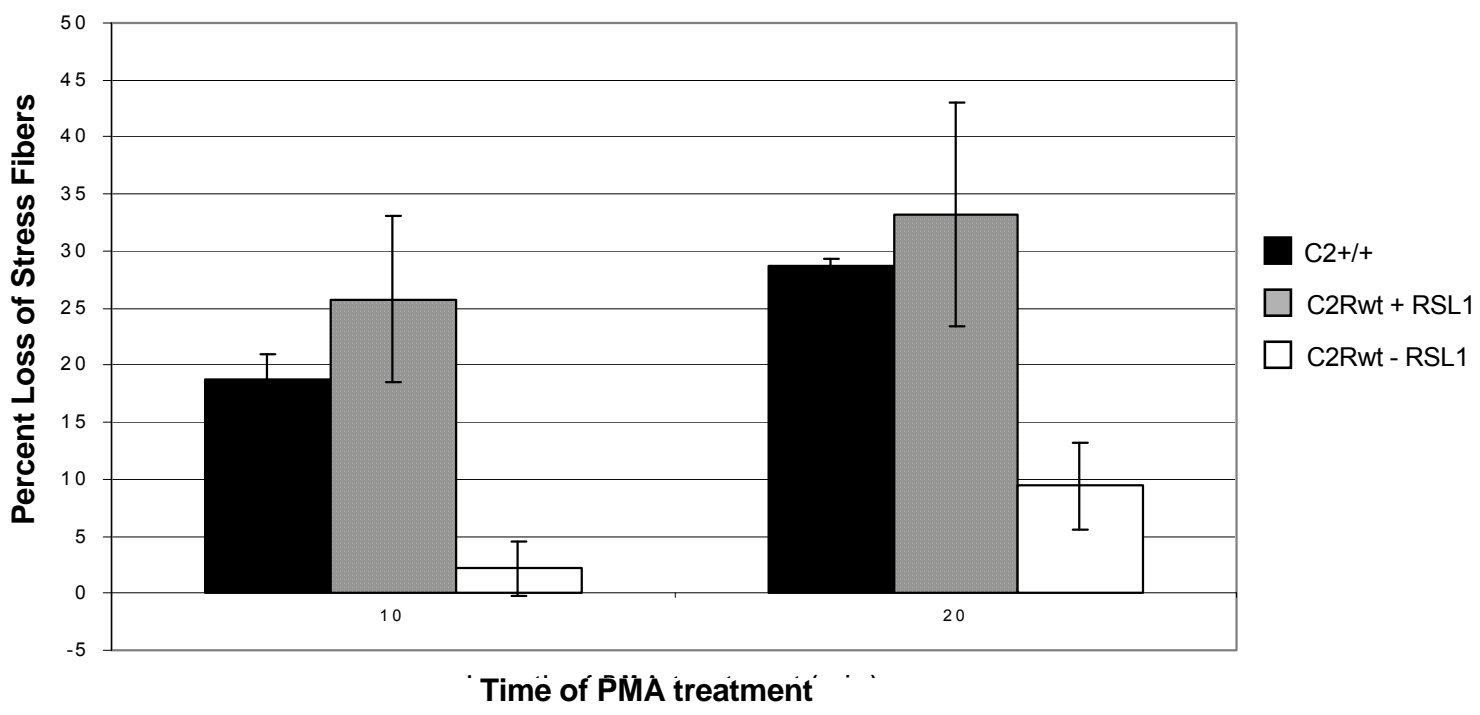

Figure 4: Caspase-2 promotes the loss of stress fibers upon PMA/TPA treatment. B) Graph representing the percentage of cells where there was a loss of stress fibers. At both 10 and 20 minutes caspase- 2 unexpressing cells demonstrate retention of stress fibers where as caspase-2 expressing cells showed a loss. 
C

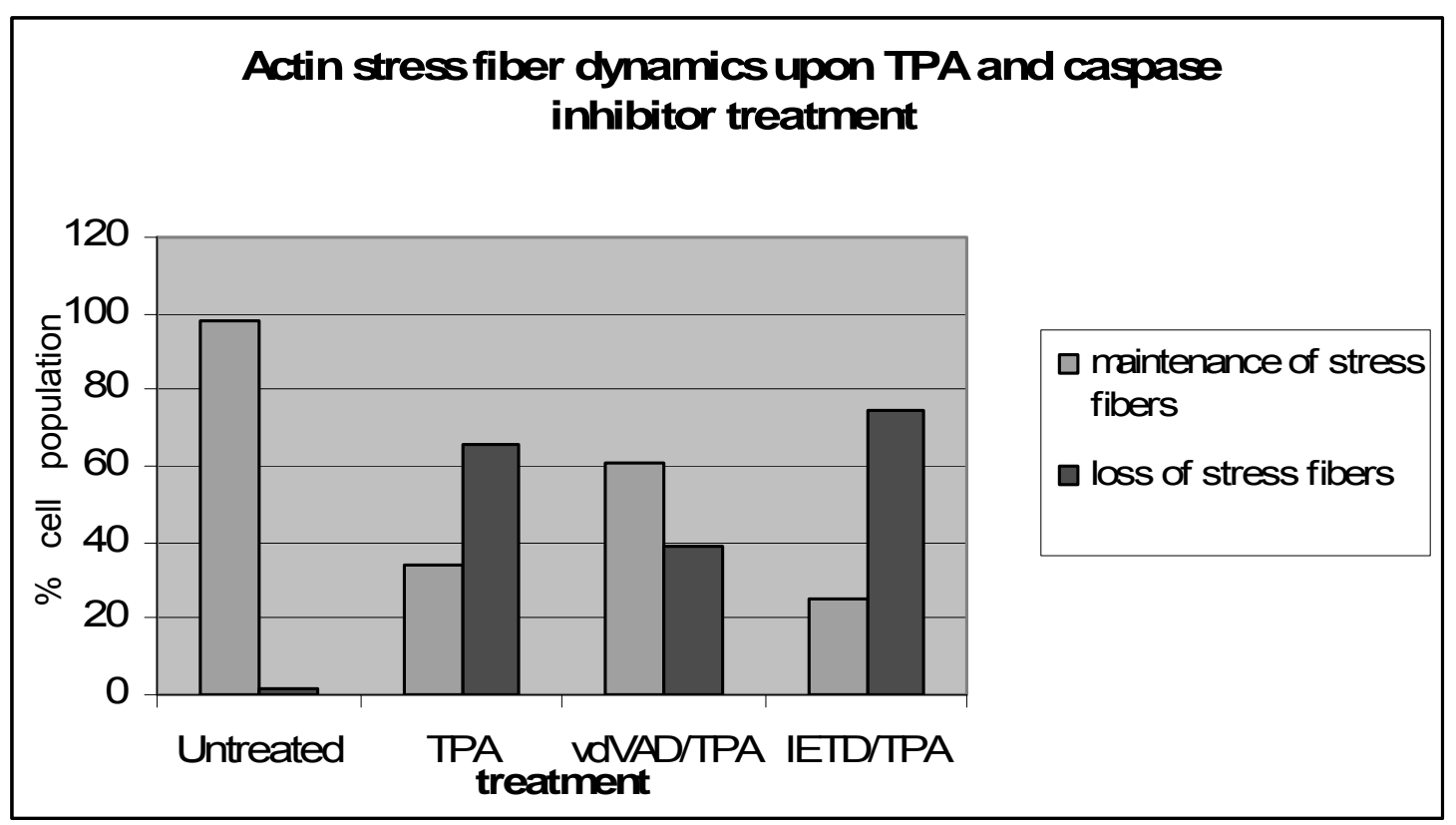

Figure 4: Caspase-2 promotes the loss of stress fibers upon PMA/TPA treatment. C) Caspase-2 inhibition (vdDAD) in wt MEFs produced similar results to caspase-2 unexpressing cells. Caspase-8 inhibition (IETD treated) produced similar results to wild type MEFs. 


\section{Figure 5}

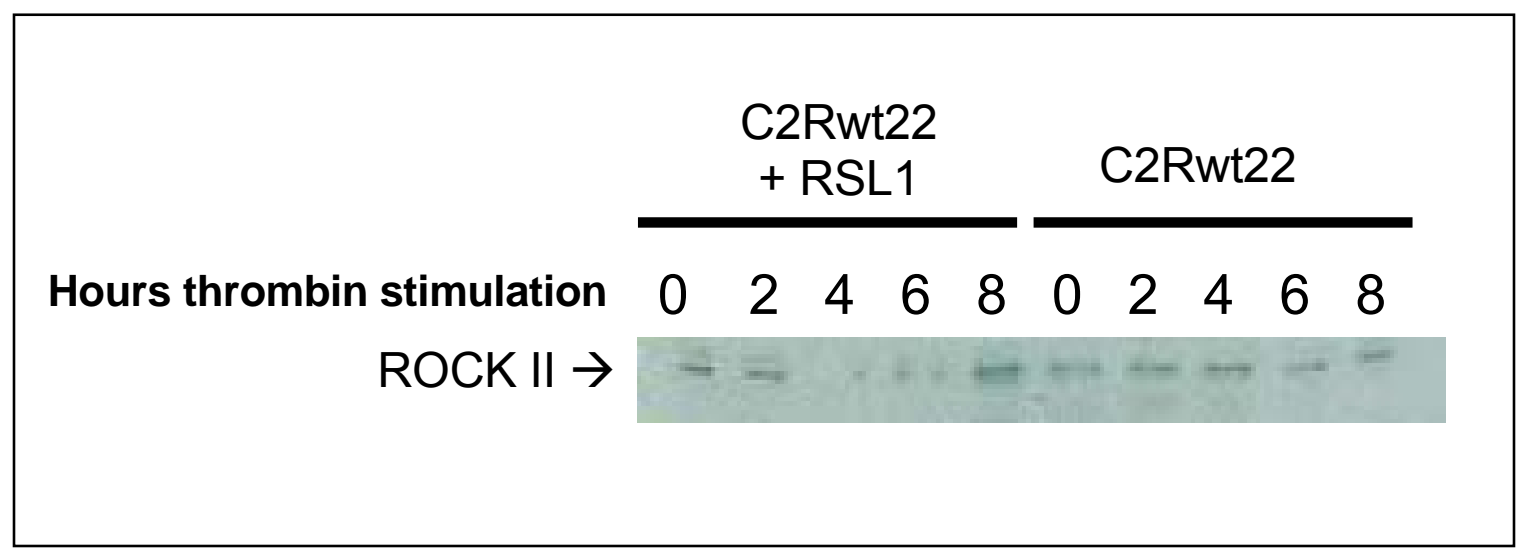

\section{ROCKII Cleavage}

Cas $2+/+$ Cas2-/-

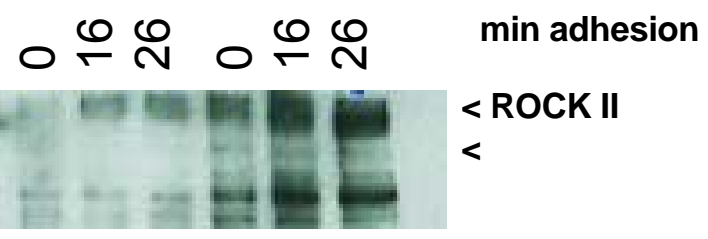

Figure 5: Caspase-2 expression does not alter ROCKII cleavage. A) Caspase-2 did not alter thrombin (1 IU/mL - Enzyme Research Laboratories Inc) stimulation induced ROCKII cleavage. Interestingly, ROCKII cleavage was absent after cleavage. This result is representative of two separate experiments. B) Cellular adhesion +/- caspase-2 did not produce a striking difference in ROCKII cleavage. 


\section{Figure 6}
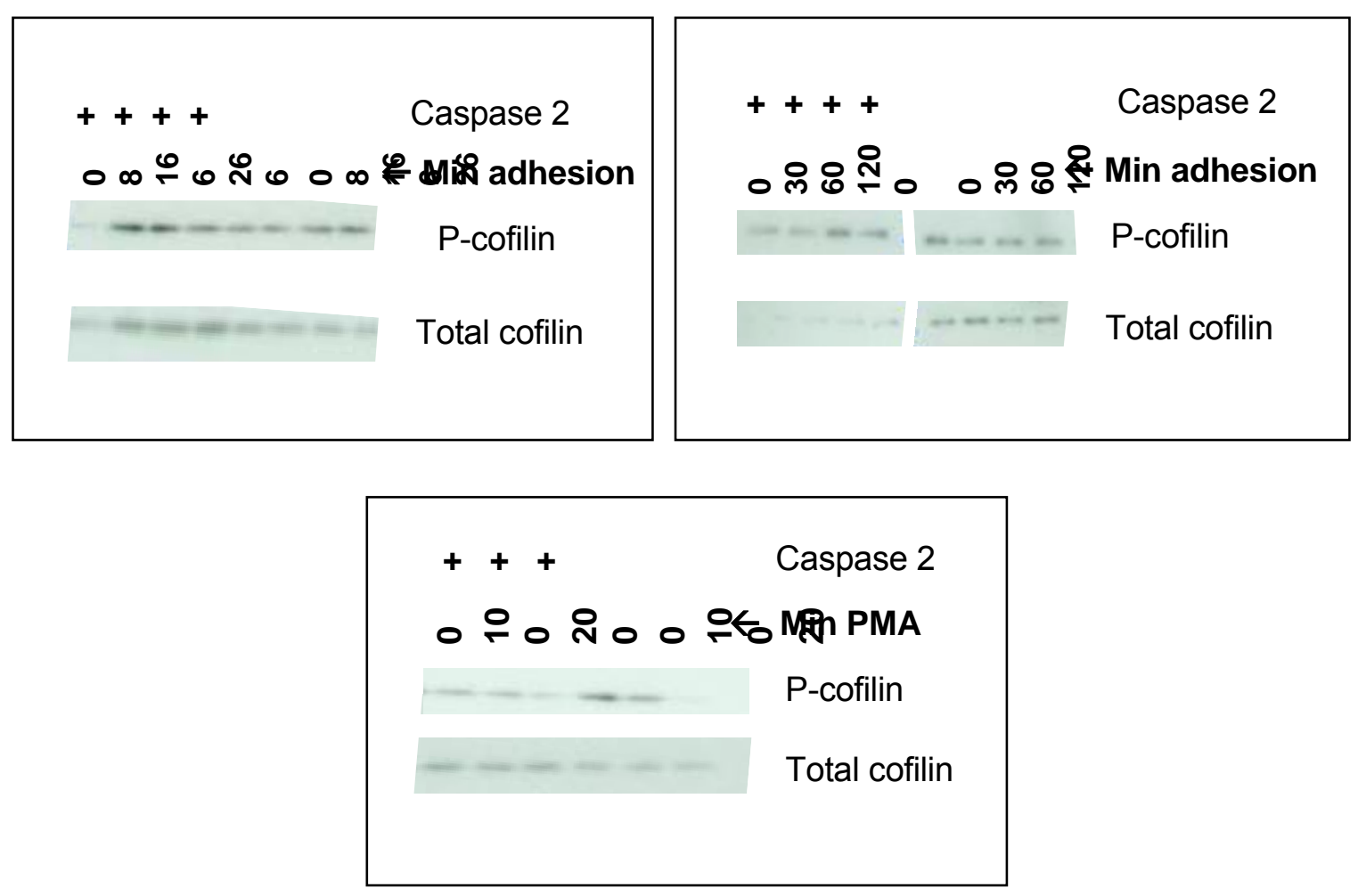

Figure 6: Cofilin phosphorylation is not substantially altered by caspase-2 expression neither in both short and long adhesion time courses nor upon PMA treatment. Upper left shows both total and phosphorylated cofilin upon a short adhesion time course (same used for p-FAK analysis), upper right shows the same during a longer adhesion time course. Lower panel shows total and phospho-cofilin upon PMA treatment. The same time course and dose of PMA were used as was done in figure 4. 


\section{Figure 7}

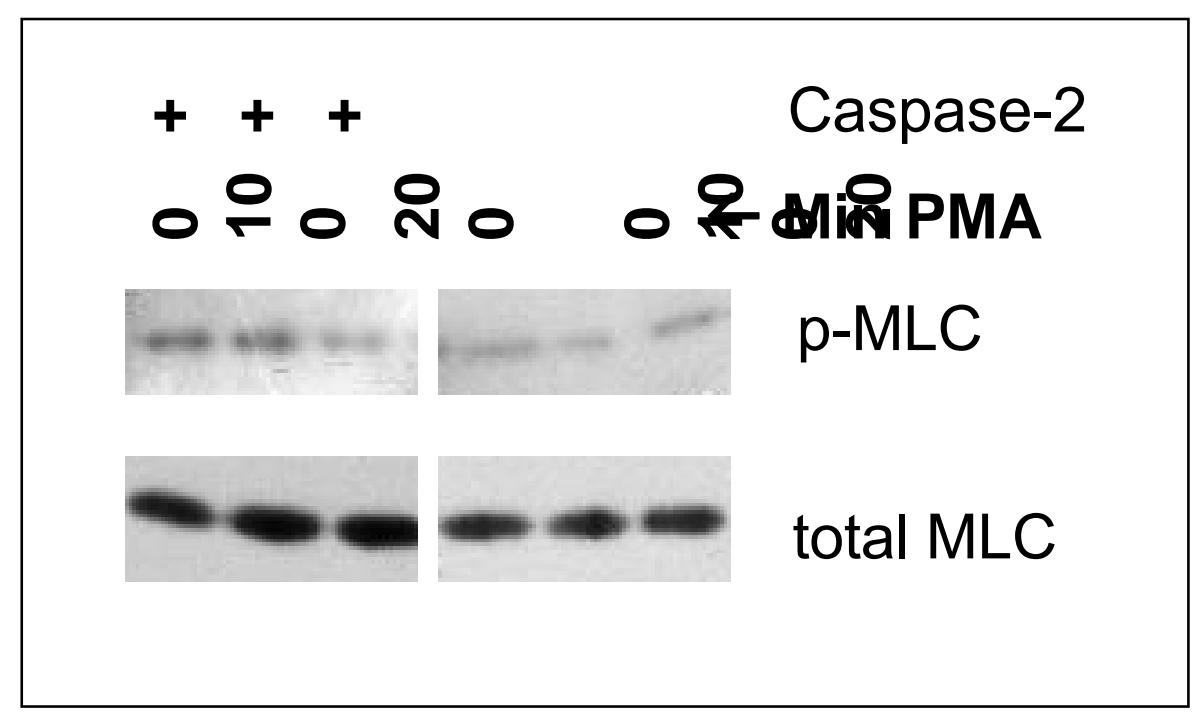

Figure 7: Phosphorylation of myosin light chain does not differ with caspase-2 expression upon PMA treatment. Wild type MEF in comparison to caspase-2 knock out MEF upon PMA treatment exhibit no change levels of phospho-MLC 


\section{Figure 8}

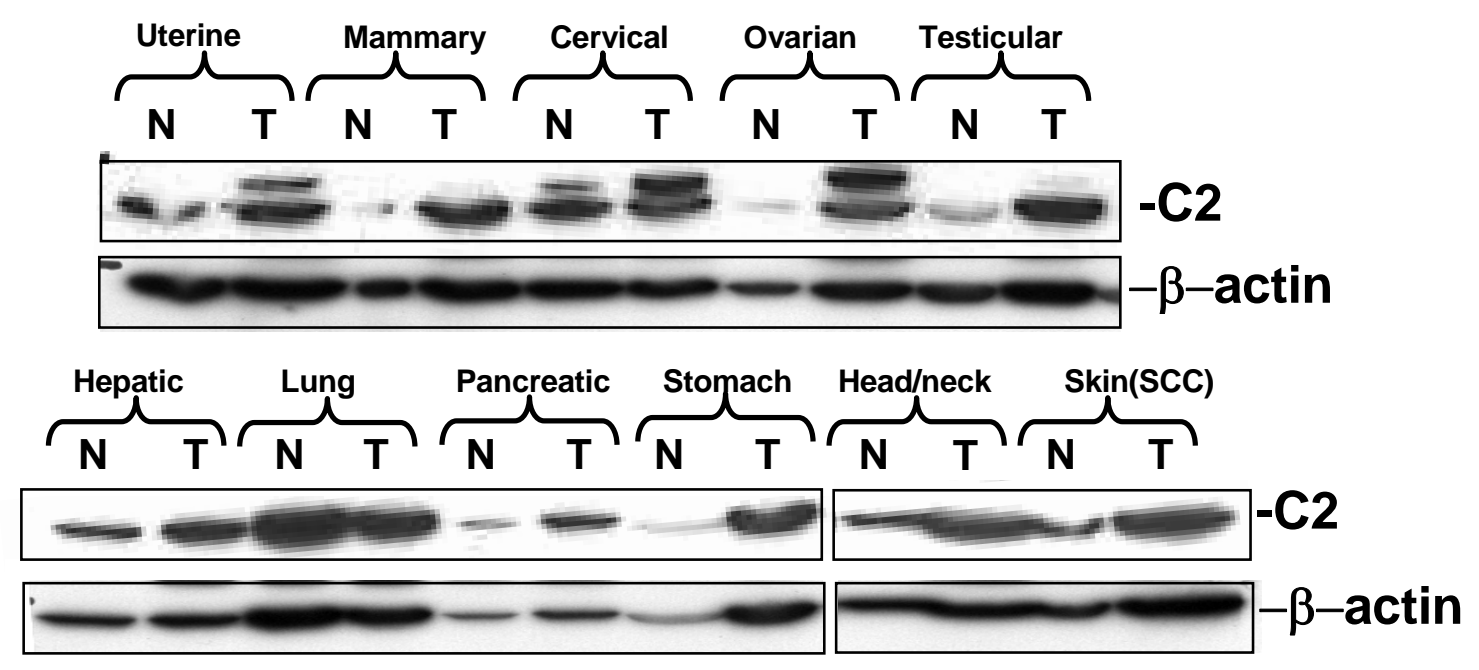

Figure 8: Tumor tissues do not lose caspase-2 expression. Genotech blot comparing normal and tumor tissue samples. Blots were probed for caspase- 2 and compared to $\beta$-actin as a loading control. 


\section{References}

1. Geisbrecht, E.R. and D.J. Montell, A role for Drosophila IAP1-mediated caspase inhibition in Rac-dependent cell migration. Cell, 2004. 118(1): p. 11125.

2. Oshima, K., et al., IKK epsilon regulates $F$ actin assembly and interacts with Drosophila IAP1 in cellular morphogenesis. Curr Biol, 2006. 16(15): p. 1531-7.

3. Kuranaga, E., et al., Drosophila IKK-related kinase regulates nonapoptotic function of caspases via degradation of IAPs. Cell, 2006. 126(3): p. 583-96.

4. Montell, D.J., A kinase gets caspases into shape. Cell, 2006. 126(3): p. 450-2.

5. Logette, E., et al., Caspase-2, a novel lipid sensor under the control of sterol regulatory element binding protein 2. Mol Cell Biol, 2005. 25(21): p. 9621-31.

6. Kong, J.Y. and S.W. Rabkin, Cytoskeletal actin degradation induced by lovastatin in cardiomyocytes is mediated through caspase-2. Cell Biol Int, 2004. 28(11): p. 781-90.

7. Kong, J.Y. and S.W. Rabkin, The association between RhoB and caspase-2: changes with lovastatin-induced apoptosis. Biochem Cell Biol, 2005. 83(5): p. 608-19.

8. Coleman, M.L., et al., Membrane blebbing during apoptosis results from caspase-mediated activation of ROCK I. Nat Cell Biol, 2001. 3(4): p. 339. 
9. Song, Y., B.Q. Hoang, and D.D. Chang, ROCK-Il-induced membrane blebbing and chromatin condensation require actin cytoskeleton. Exp Cell Res, 2002. 278(1): p. 45-52.

10. Sapet, C., et al., Thrombin-induced endothelial microparticle generation: identification of a novel pathway involving ROCK-Il activation by caspase-2. Blood, 2006. 108(6): p. 1868-1876.

11. Totsukawa, G., et al., Distinct Roles of ROCK (Rho-kinase) and MLCK in Spatial Regulation of MLC Phosphorylation for Assembly of Stress Fibers and Focal Adhesions in 3T3 Fibroblasts. J. Cell Biol., 2000. 150(4): p. 797-806.

12. Huang, T.Y., C. DerMardirossian, and G.M. Bokoch, Cofilin phosphatases and regulation of actin dynamics. Curr Opin Cell Biol, 2006. 18(1): p. 26-31.

13. Yoneda, A., H.A. Multhaupt, and J.R. Couchman, The Rho kinases I and II regulate different aspects of myosin II activity. J Cell Biol, 2005. 170(3): p. 44353.

14. Maekawa, M., et al., Signaling from Rho to the actin cytoskeleton through protein kinases ROCK and LIM-kinase. Science, 1999. 285(5429): p. 895-8.

15. O'Reilly, L.A., et al., Caspase-2 is not required for thymocyte or neuronal apoptosis even though cleavage of caspase-2 is dependent on both Apaf-1 and caspase-9. Cell Death Differ, 2002. 9(8): p. 832-41.

16. Brandt, D., et al., Protein Kinase C Induces Actin Reorganization via a Src- and Rho-dependent Pathway. J. Biol. Chem., 2002. 277(23): p. 20903-20910. 
17. Brandt, D.T., et al., Protein Kinase C\{delta\} Induces Src Kinase Activity via Activation of the Protein Tyrosine Phosphatase PTP\{alpha\}. J. Biol. Chem., 2003. 278(36): p. 34073-34078.

18. Arthur, W.T., N.K. Noren, and K. Burridge, Regulation of Rho family GTPases by cell-cell and cell-matrix adhesion. Biol Res, 2002. 35(2): p. 239-46.

19. Baliga, B.C., S.H. Read, and S. Kumar, The biochemical mechanism of caspase-2 activation. Cell Death Differ, 2004. 11(11): p. 1234-41.

20. Wheeler, A.P. and A.J. Ridley, Why three Rho proteins? RhoA, RhoB, RhoC, and cell motility. Exp Cell Res, 2004. 301(1): p. 43-9.

21. Koch, G., et al., Role of Rho Protein in Lovastatin-Induced Breakdown of Actin Cytoskeleton. J Pharmacol Exp Ther, 1997. 283(2): p. 901-909.

22. Ho, L.H., et al., Caspase-2 is required for cell death induced by cytoskeletal disruption. Oncogene, 2008.

23. Holleman, A., et al., Decreased PARP and procaspase-2 protein levels are associated with cellular drug resistance in childhood acute lymphoblastic leukemia. Blood, 2005. 106(5): p. 1817-23.

24. Bullen, T.F., et al., Characterization of epithelial cell shedding from human small intestine. Lab Invest, 2006. 86(10): p. 1052-63.

25. Troy, C.M., et al., Death in the balance: alternative participation of the caspase2 and -9 pathways in neuronal death induced by nerve growth factor deprivation. J Neurosci, 2001. 21(14): p. 5007-16. 
26. Kumar, S., Caspase function in programmed cell death. Cell Death Differ, 2007. 14(1): p. 32-43.

27. Chun, H.J., et al., Pleiotropic defects in lymphocyte activation caused by caspase-8 mutations lead to human immunodeficiency. Nature, 2002. 419(6905): p. 395-9.

28. Launay, S., et al., Vital functions for lethal caspases. Oncogene, 2005. 24(33): p. 5137-48.

29. Tibbetts, M.D., L. Zheng, and M.J. Lenardo, The death effector domain protein family: regulators of cellular homeostasis. Nat Immunol, 2003. 4(5): p. 404-9.

30. Wang, J., et al., Caspase-10 is an initiator caspase in death receptor signaling. Proc Natl Acad Sci U S A, 2001. 98(24): p. 13884-8.

31. Yoo, N.J., et al., Loss of caspase-2, -6 and -7 expression in gastric cancers. Apmis, 2004. 112(6): p. 330-5.

32. Waddell, D.R., K. Duffy, and G. Vogel, Cytokinesis is defective in Dictyostelium mutants with altered phagocytic recognition, adhesion, and vegetative cell cohesion properties. J. Cell Biol., 1987. 105(5): p. 2293-2300.

33. Robinson, D.N. and J.A. Spudich, Towards a molecular understanding of cytokinesis. Trends Cell Biol, 2000. 10(6): p. 228-37. 
Chapter 5

\section{Overall Discussion}




\section{Discussion}

The field of non-apoptotic caspases is an area that until recently has not received its due attention. The executioners of the cell are being found to have roles in development, differentiation and cytoskeletal dynamics. The balance of life and death is often challenged in the occurrence of cancer. Our studies demonstrate signaling pathways that, in the context of cancer cells resistant to apoptosis, may actually contribute to tumor progression. Specifically, our studies demonstrate the roles of caspase- 8 and caspase- 2 in cell adhesion, migration, and cytoskeletal response to phorbol esters. Our work, along with others, highlights the impact that the expression of these caspases can have in normal cells and in cancer progression along with how their expression can influence potential therapy options.

Caspase-8 may influence cellular adhesion/migration in a cell context dependent mechanism or via multiple pathways. In chapter 2, we examined caspase-8's role in adhesion and migration and suggested a calpain dependent mechanism. In chapter 3 , we showed that caspase-8 interacts directly with p85 in a caspase-8 phospho-Y380 dependent manner to promote adhesion, migration, and Rac activation.

Discovering the relative contributions of these apparently distinct mechanisms, and possible connections between them, is the focus of current and planned investigations in the lab. Caspase-8 may be acting via dual mechanisms to promote motility. In our first study, many experiments were peformed using knock out MEFs. Mice lack a very important factor - the Y380 residue [1]. Yet, in these cells the presence of murine 
caspase-8 still promotes cellular migration. The ability to promote calpain activation and Erk activity (and thus the proposed pathway of caspase-8 $\rightarrow$ MEKK1 $\rightarrow$ Erk $\rightarrow$ calpain) is not limited to murine cells (Chapter 2) making this mechanism of action likely to be involved in the promotion of migration/adhesion.

Erk activation is involved in many aspects of cell migration, including calpain activation and phosphorylation of FAK, MLCK, and paxillin (reviewed in the introduction and [2]). In cell systems where the proposed caspase-8/MEKK1/Erk/calpain mechanism predominates (eg. mouse fibroblasts), the catalytic activity of caspase- 8 would be required for cleavage of MEKK1 and subsequent stimulation of Erk. Consistent with the need for catalytic activity, a recent publication highlights basal caspase activity that promotes glioblastoma invasion and migration [3]. In this report, caspase activity is required for invasion and migration. Our experiments where we reconstituted Rheoswitch-inducible expression of catalytically active vs. inactive caspase-8 mutants in knockout MEFs and assayed for cell migration have produced ambiguous results, which require further investigation. Another strategy that we are going to employ for testing the catalytic requirement of caspase-8 is to reconstitute MEKK1-knockout fibroblasts with either wild-type or caspase uncleavable mutant forms of MEKK1 to be assayed for their ability to restore cell migration and adhesion; these experiments are underway in our laboratory.

If caspase-8 catalytic activity and/or MEKK1 cleavage is not found to be required in certain cell lines, another possibility, in addition to the caspase-8-p85 interaction 
discussed extensively herein, for promotion of migration is that additional proteins containing $\mathrm{SH} 2$ domains may interact with caspase-8 $\mathrm{Y} 334$, a residue which is also phosphorylated [4]. Proteins that may interact with this phosphorylated residue and others that may interact with phospho-Y380 are expected to be identified by proteomic analysis.

In support of capsase-8 acting via multiple mechanisms to promote cell migration, CD95 has been connected to migration and invasion utilizing similar mechanisms. CD95 (Fas/Apo-1), is a death receptor responsible for the formation of the death inducing signaling complex (DISC) [5] to promote apoptosis. CD95 signaling is nonapoptotically associated with invasion and motility through NF-kappaB, Erk and caspase-8 signaling [6] upon stimulation by FasL. In the case of CD95, another paper in the literature supports our idea that caspase-8 autoactivation is not required for its promotion of cell motility: the anti-APO-1 antibody, when added to cells both promotes motility and Erk activation but does not induce apoptosis or processing of procaspase-8 [7]. Note that this result does not say whether catalytic activity of caspase- 8 is required or not, as procaspase- 8 can be catalytically active under some circumstances [8]. A recent publication challenges the involvement of caspase-8 in this process. They state that Fas-induced cell migration can occur in some lines independent of caspase-8 recruitment to the DISC, suggesting that in some contexts, Fas may stimulate migration independently of caspase-8 [9] (although this was based on weak evidence only -lack of effect of a caspase-8 inhibitor peptide and lack of recruitment of caspase-8 to the DISC). 
Many cell types, including breast and pancreatic cancer cells, have significant caspase-8 activity that could potentially affect cell behavior under non-apoptotic conditions $[10,11]$. This increased activity may be caused by elevated soluble FASL in some cancers, especially those treated with chemotherapeutic agents [12, 13]. The maintenance of caspase-8 in many tumor types (Chapter 3, Figure 1), along with increased caspase-8 activity brings up an interesting dilemma in selection of cancer therapies. Any therapy (eg TRAIL [14-18]) that activates caspase-8 in apoptosisresistant tumor cells, whether by accident or design, has the potential of stimulating tumor cell migration and invasion, undesirable effects.

Caspase-2, another initiator caspase, examined in Chapter 4, is important for cellular adhesion and for stress fiber break down upon phorbol-ester treatment. While initial hypotheses to identify the mechanism of caspase-2's actions fell short, they did provide reason to take a second look at the proposed mechanisms as well as insight to other potential avenues for caspase-2 action.

According to the hypothesis that caspase-2 influences ROCK cleavage, caspase-2's catalytic activity would be required for its downstream cytoskeletal effects. The catalytic activity of caspase-2 appears to be required for effect upon stress fiber breakdown, because a caspase-2 inhibitor peptide suppressed stress fiber breakdown. This result is consistent with the dependence of ROCKII cleavage on caspase- 2 that we observed in our preliminary data. It is also possible that cellular adhesion and stress fiber dynamics are controlled by caspase- 2 by multiple 
mechanisms or forms of caspase-2. Should caspase-2 interact with RhoB [19], its response may be through this interaction, rather than its catalytic activity.

It is likely that other mechanisms are involved in the regulation of caspase-2 in cytoskeletal dynamics. The data on cofilin and MLC phosphorylation and RhoA activation do not suggest that caspase- 2 is influencing their pathways of activation (Chapter 4 and unpublished data YJC). We are currently in the process of developing a caspase-2-FLAG-S-tag construct to be expressed in knockout MEFs in order to perform proteomic analysis for novel caspase-2 interactions, similar to what is being done with caspase-8. Elucidation of novel interactions may be able to explain how caspase-2 is regulating cellular adhesion and stress fiber breakdown.

Caspase-2 has recently been associated with cytoskeletal disruption induced apoptosis [20], as discussed in Chapter 4. It is possible that a lack of caspase-2 expression allows cytoskeletal abnormalities to manifest instead of undergoing apoptosis, which would be the case in caspase-2 expressing cells. It will be interesting to see, as this project is continued, whether caspase-2 is able to act independently of apoptosis.

Alterations of caspase-2 expression levels also exist in gastric cancer and leukemia $[21,22]$. According to a screen of normal vs. tumor tissue samples, caspase-2 expression is often maintained in tumors (Chapter 4, Fig 8), making it an interesting 
candidate for a non-apoptotic function involved in tumor progression, analogous in some ways to the caspase-8 phenomenon.

Our work with caspase-8 and caspase- 2 has made a notable contribution to the field of non-apoptotic caspase functions. Our work with caspase-8 was the first to demonstrate its role in cell motility and adhesion, as well as the first reported procaspase function, via its interaction with p85. While the experiments dealing with caspase-2 are in the early stages of its development it will be interesting to see how the field progresses as our lab and others continue to contribute to its advancement. 


\section{References}

1. Cursi, S., et al., Src kinase phosphorylates Caspase-8 on Tyr380: a novel mechanism of apoptosis suppression. Embo J, 2006. 25(9): p. 1895-905.

2. Huang, C., K. Jacobson, and M.D. Schaller, MAP kinases and cell migration. J Cell Sci, 2004. 117(Pt 20): p. 4619-28.

3. Gdynia, G., et al., Basal caspase activity promotes migration and invasiveness in glioblastoma cells. Mol Cancer Res, 2007. 5(12): p. 1232-40.

4. Rush, J., et al., Immunoaffinity profiling of tyrosine phosphorylation in cancer cells. Nat Biotechnol, 2005. 23(1): p. 94-101.

5. Kischkel, F.C., et al., Cytotoxicity-dependent APO-1 (Fas/CD95)-associated proteins form a death-inducing signaling complex (DISC) with the receptor. Embo J, 1995. 14(22): p. 5579-88.

6. Barnhart, B.C., et al., CD95 ligand induces motility and invasiveness of apoptosis-resistant tumor cells. Embo J, 2004. 23(15): p. 3175-85.

7. Lee, K.H., et al., The role of receptor internalization in CD95 signaling. Embo J, 2006. 25(5): p. 1009-23.

8. Boatright, K.M., et al., A unified model for apical caspase activation. Molecular Cell, 2003. 11(2): p. 529-41.

9. Kleber, S., et al., Yes and PI3K bind CD95 to signal invasion of glioblastoma. Cancer Cell, 2008. 13(3): p. 235-48. 
10. He, L., et al., Monitoring caspase activity in living cells using fluorescent proteins and flow cytometry. Am J Pathol, 2004. 164(6): p. 1901-13.

11. Yang, L., et al., Coexistence of high levels of apoptotic signaling and inhibitor of apoptosis proteins in human tumor cells: implication for cancer specific therapy. Cancer Res, 2003. 63(20): p. 6815-24.

12. Fulda, S., et al., Chemosensitivity of solid tumor cells in vitro is related to activation of the CD95 system. International Journal of Cancer, 1998. 76(1): p. 105-14.

13. Owen-Schaub, L., et al., Fas and Fas ligand interactions in malignant disease. Int J Oncol, 2000. 17(1): p. 5-12.

14. Nagane, M., H.J. Huang, and W.K. Cavenee, The potential of TRAIL for cancer chemotherapy. Apoptosis, 2001. 6(3): p. 191-7.

15. Wu, X.X., et al., Doxorubicin enhances TRAIL-induced apoptosis in prostate cancer. International Journal of Oncology, 2002. 20(5): p. 949-54.

16. Wajant, H., K. Pfizenmaier, and P. Scheurich, TNF-related apoptosis inducing ligand (TRAIL) and its receptors in tumor surveillance and cancer therapy. Apoptosis, 2002. 7(5): p. 449-59.

17. Held, J. and K. Schulze-Osthoff, Potential and caveats of TRAIL in cancer therapy. Drug Resistance Updates, 2001. 4(4): p. 243-52.

18. Fricker, J., On the TRAIL to a new cancer therapy. Molecular Medicine Today, 1999. 5(9): p. 374. 
19. Kong, J.Y. and S.W. Rabkin, The association between RhoB and caspase-2: changes with lovastatin-induced apoptosis. Biochem Cell Biol, 2005. 83(5): p. 608-19.

20. Ho, L.H., et al., Caspase-2 is required for cell death induced by cytoskeletal disruption. Oncogene, 2008.

21. Holleman, A., et al., Decreased PARP and procaspase-2 protein levels are associated with cellular drug resistance in childhood acute lymphoblastic leukemia. Blood, 2005. 106(5): p. 1817-23.

22. Yoo, N.J., et al., Loss of caspase-2, -6 and -7 expression in gastric cancers. Apmis, 2004. 112(6): p. 330-5. 


\section{Index}




\title{
Caspase-8 Promotes Cell Motility and Calpain Activity under Nonapoptotic Conditions
}

\author{
Brooke Helfer, ${ }^{1}$ Bernard C. Boswell, ${ }^{1}$ Darren Finlay, ${ }^{2}$ Angel Cipres, ${ }^{2}$ Kristiina Vuori, \\ Tae Bong Kang, ${ }^{3}$ David Wallach, ${ }^{3}$ Andrea Dorfleutner,' Jill M. Lahti, \\ Daniel C. Flynn, ${ }^{1}$ and Steven M. Frisch ${ }^{1}$ \\ 'Mary Babb Randolph Cancer Center and Department of Biochemistry, West Virginia University, Morgantown, West Virginia; \\ 'The Burnham Institute, La Jolla, California; 'Department of Biological Chemistry, Weizmann Institute of Science, Rehovot, Israet \\ and 'Department of Genetics and Tumor Cell Biology, St. Jude Children's Research Hospital, Memphis, Tennessee
}

\begin{abstract}
Significant caspase- 8 activity has been found in normal and certain tumor cells, suggesting that caspase- 8 possesses an alternative, nonapoptotic function that may contribute to tumor progression. In this article, we report that caspase8 promotes cell motility. In particular, caspase- 8 is required for the optimal activation of calpains, Rac, and lamellipodial assembly. This represents a novel nonapoptotic function of caspase- 8 acting at the intersection of the caspase- 8 and calpain proteolytic pathways to coordinate cell death versus cell motility signaling. (Cancer Res 2006; 66(8): 4273-8)
\end{abstract}

\section{Introduction}

Many cell types, including breast and pancreatic cancer cells, have significant caspase- 8 activity that could potentially affect cell behavior under nonapoptotic conditions $(1,2)$. This increased activity may be caused by elevated soluble FASL in some cancers, especially those treated with chemotherapeutic agents $(3,4)$. Heterodimerization with overexpressed tumor cell c-FLIP also activates caspase- $8(5,6)$, elevating basal activity. At present, the possible significance of this constitutive caspase- 8 activity is unknown. Diverse nonapoptotic functions are emerging for caspases, including differentiation, proliferation, and promotion of the immune response (7-11), suggesting that constitutive caspase activity may be relevant to normal and tumor biology.

Cell migration is a critical contributor to tumor invasion and metastasis (12). Interestingly, roles for certain caspases in cell migration have been suggested. The Drosophila caspase, DRONC, a homologue of mammalian caspase-2 and caspase-9, modulates developmental cell migration due to an interaction of its endogenous inhibitor, DIAP, with the Rac pathway (13). Caspase- 8 gene knockouts are early-embryonic lethal in mice (14), mainly because they fail to assemble a functional circulatory system, suggesting a defect in endothelial cell migration; other possible cell migratory defects occur in the context of tissue-specific knockouts (15). Moreover, several adhesion/motility-related structural and signaling proteins are cleaved by caspases during apoptosis (16-32). Corresponding cleavage of these substrates during nonapoptotic processes, such as cell migration, may provide a mechanism for

Note: Supplementary data for this article are available at Cancer Research Online (https//cancerres.aacriournals.org/).

Requests for reprints: Steven M. Frisch, Mary Babb Randolph Cancer Center and Department of Biochemistry, West Virginia University, 1 Medical Center Drive, POB 9300, Morgantown WV 26506. Phone: 304-293-2980; E-mail: sfrischghsc.wvu.edu. Q2006 American Association for Cancer Research

doi:10.1158/0008-5472.CAN-05-4183 caspases to control the tumover or alter the activation status of these substrates.

Calpains are implicated in several aspects of cell migration, including adhesion turnover, lamellipodial extension/retraction cycles, detachment of the trailing edge of the cell, and activation of rac (reviewed in ref. 33). Knockout of the mouse calpain-4 gene, which encodes a regulatory subunit that is requined for the in vivo stability of the ubiquitous calpain-1 and calpain-2, generates an embryonic-lethal phenotype (34) that is similar in timing and cause of death to that of caspase- 8 knockout mice. (Note, however, that the substantial amount of cell migration occurring before day 10 of development in these and caspase-8-knockout mice occurs normally, indicating that this cell migration is independent of these proteases, perhaps due to the compensation by or predominance of other migration signaling pathways in early embryonic cell types responding to a unique configuration of growth factors/chemoattractants.) Consistent with their cell migration function, calpains provide an important tumor-promoting function (33, 35-37) and tumor cells often have increased calpain activity $(35,36,38-41)$. A role of caspase-8 and/or a functional relationship between caspase- 8 and calpains in tumor prognession has yet to be addressed.

In this report, we show that caspase- 8 regulates cell motility and calpain activity. Cells that lack caspase- 8 have reduced cell motility and calpain activity as well as other manifestations of calpain deficiency, such as reduced Rac activation, lamellipodial assembly, and fidelity of cytokinesis. This reveals a potential nonapoptotic role of caspase- 8 in tumor progression.

\section{Materials and Methods}

Cell Lines

Mouse embryonic fibroblasts (MEF) derived from caspase-8-null, caspase-3-null, or isogenic wild-type control mice were previously characterized $(14,42)$. WTNR6 cells $(43)$ were provided by Alan Wells. To generate derivatives expressing the cowpox vinus-derived caspase8 inhibitory protein $\mathrm{crmA}$ (44), the hemagglutinin-tagged $\mathrm{crmA}$ gene was retrovirally transduced using the vector MSCV-IRES-puro in the gp $2+293$ cell packaging system (Invitrogen, Carlsbad, CA). NB7 cells that were infected with emply green fluorescent protein retrovinus or rescued with human caspase- 8 retrovirus (45) were provided by Jill Lahti. MDA-MB231 (American Type Culture Collection, Rockville, MD) were transduced with crmA as described above. Cells were maintained in DMEM (MEF, MDAMB231), advanced MEM (WTNR6), and RPMI 1640 (NB7), supplemented with $10 \%$ fetal bovine serum (FBS) and $1 \times$ penicillin-streptomycinglutamine (Invitrogen).

Cell lines with inducible caspase-8 expression were generated using the Rheoswitch system (www.neb.com). Caspase- ${ }^{-1-}$ MEFs (W102) were coelectroporated with a $4: 1$ ratio of the Rheoswitch activator/repressorexpressing plasmid, NEBR-RI:pcDNA3.1hyg and colonies were selected 
in $500 \mu \mathrm{g} / \mathrm{mL}$ hygromycin. Colonies were expanded and screened for expression of the VP16 moiety of the hybrid activator protein by Western blotting and for induction of luciferase activity after transient transfection of luciferase/pNEBR-X1. Wild-type mouse caspase-8 cDNAs were subcloned into the pNEBR-XI target plasmid and coelectroporated in a 4:1 ratio with pBABE-puro. Colonies were selected in $5 \mu \mathrm{g} / \mathrm{mL}$ puromycin and screened for caspase-8 expression by Western blotting with the anti-mouse-caspase-8 antibody 1 G12 (Alexis, San Diego, CA) after induction with the compound RSLI. A Western blot of the induction time course is shown in Fig. 1. The level of caspase-8 expression at 24 hours of induction was $\sim 30 \%$ to $50 \%$ of the level in wild-type MEFs (data not shown).

\section{Production of Calpastatin-TAT Peptide}

Double-stranded oligonucleotides corresponding the active calpaininhibitory domain of calpastatin (46) were subcloned into the vector pET28b-TATv1 (provided by Steve Dowdy). The fusion protein was expressed in Escherichia coli BL21 with 2 hours of isopropyl-L-thio-B-D-galactopyranoside induction; bacteria were lysed in buffer $\mathrm{Z}[6 \mathrm{~mol} / \mathrm{L}$ urea/100 mmol/L $\mathrm{NaCl} / 20 \mathrm{mmol} / \mathrm{L} . \mathrm{HEPES}(\mathrm{pH} 8)]$ and purified on a nickel-chelate resin (Qiagen, Valencia, CA) in the same buffer containing $20 \mathrm{mmol} / \mathrm{L}$ imidazole. The protein was eluted using a $40 \mathrm{mmol} / \mathrm{L}$ interval imidazole step gradient, and pooled fractions were chromatographed on a BioRex 70 column in $50 \mathrm{mmol} / \mathrm{L} \mathrm{NaCl} / 20 \mathrm{mmol} / \mathrm{L}$ HEPES and eluted with $1 \mathrm{~mol} / \mathrm{L} \mathrm{NaCl} /$ $20 \mathrm{mmol} / \mathrm{L}$ HEPES; pooled fractions were dialyzed against $0.5 \mathrm{~mol} / \mathrm{L} \mathrm{NaCl} /$ $20 \mathrm{mmol} / \mathrm{L}$ HEPES and frozen.

\section{Cell Motility Assays}

Method 1. Electrical cell-substratum impedance sensing. Cells were plated on the chambers $\left(\sim 1 \mathrm{~cm}^{2}\right)$ of a single-electrode electrical cellsubstratum impedance sensing (ECIS) arrays (Applied Biophysics, Troy, NY; www.biophysics.com) that were precoated with fibronectin. After growing the cells to confluence, equal coverage of the electrodes was checked by measuring the initial impedance value (averages were 1,924 ohms for caspase $-8^{-/-}$cells and 2,598 for caspase- $8^{+/+}$cells). The cells attached to the small electrode disc $(0.25-\mathrm{mm}$ diameter) were selectively ablated by applying a $4 \mathrm{~V}$ pulse for 10 seconds, causing the impedance drop seen at 0 hours. The impedance increase due to the surrounding cells migrating back onto the electrode was then monitored at $40 \mathrm{kHz}$ frequency over the indicated time course; migration was in the presence of $10 \%$ serum and was carried out in triplicate chambers with one additional "unwounded" chamber serving as a negative control. Data were exported to Excel for calculation of slopes.

Method 2. Conventional wound healing. Cells were grown to confluence on fibronectin-coated coverslips or $35 \mathrm{~mm}$ Mattek dishes, serum-starved overnight $(0.5 \%$ serum), and the monolayer was wounded with a pipette tip; epidermal growth factor (EGF; $10 \mathrm{nmol} / \mathrm{L}$ ) was then added. The wound was photographed at zero time and at the indicated time points using vertical and horizontal alignment marks made on the plastic well with a syringe needle. Three wound areas were traced and measured using the Axiovision software, averaged, and represented here as the percentage wound closure. Alternatively, 1 hour after attachment, a wound was generated, unattached cells were washed off, and videomicroscopy was done on the dish, which was incubated on a heated stage in $\mathrm{CO}_{2}$ -

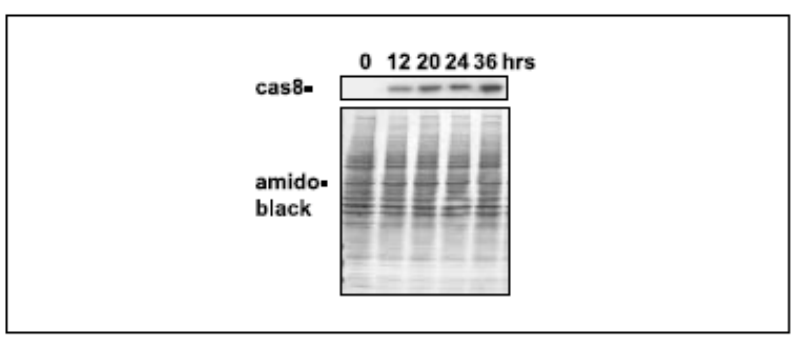

Figure 1. Inducible expression of caspase-8. independent medium/CCM1 medium (1:1). In experiments using the Rheoswitch-inducible cell lines, cells were induced for 24 hours in the presence of $500 \mathrm{nmol} / \mathrm{L}$ RSL1 before wounding.

Method 3. Single-cell motility assays. Cells were plated onto fibronectin at low density for 1 hour in the absence of senum, restimulated with serum, and recorded by videomicroscopy for 10 hours. The paths traveled by seven individual cells were traced and the total distance traveled by each cell was computed using the Axiovision software (interactive measurement module).

Method 4. Transwell/chemotaxis assays. Cell migration assays were done with modified Boyden chambers, $6.5 \mathrm{~mm}$ diameter, $8 \mathrm{~mm}$ pore size (Transwell from Costar Corp., Acton, MA) according to the protocol of the manufacturer. Briefly, after serum starvation of the MEF cells, they were harvested with trypsin and quenched with Soybean trypsin inhibitor (Calbiochem, San Diego, CA). The lower compartment of the migration chamber was filled with 3T3-fibroblast conditioned medium and $6 \times 10^{4}$ cells were suspended in DMEM/10 mmol/L HEPES $/ 0.5 \%$ bovine serum albumin (BSA) $/ 1 \%$ penicillin-streptomycin and added to the upper compartment of the migration chamber. Migration chambers were incubated for 4 hours at $37^{\circ} \mathrm{C}$ in $5 \% \mathrm{CO}_{2}$. After removal of stationary cells from the upper side of the membrane, migrated cells were fixed in $3.7 \%$ paraformaldehyde in PBS and stained with $1 \%$ crystal violet in $2 \%$ ethanol. Membranes were extensively washed, the dye was eluted with $10 \%$ acetic acid, and absorbance was measured at $600 \mathrm{~nm}$. Results are expressed as percentage of migrated cells relative to a control in which total adherent cell absorbance was measured.

\section{Calpain Assays}

Method 1. Plate-reading fluorimetry using succinyl-Leu-Leu-ValTyr-7-amido-4-methylcoumarin substrate. Cells were seeded into 96 well plates $\left(10^{4}\right.$ per well) and allowed to attach overnight (47). When EGF stimulation was used, the cells were serum starved (0.5\% FBS) for 8 to 12 hours before treatment. Five minutes before addition of EGF, the medium was aspirated and replaced with $100 \mu \mathrm{L}$ phenol red-free medium containing $0.5 \%$ FBS with $50 \mu \mathrm{mol} / \mathrm{L}$ succinyl-Leu-Leu-Val-Tyr-7-amido4-methylcoumarin (Suc-LLVY-AMC; Calbiochem). At time zero, $10 \mathrm{nmol} / \mathrm{L}$ EGF was added. The plate was then loaded into the prewarmed $\left(37^{\circ} \mathrm{C}\right)$ plate reader (Gemini XPS, Molecular Devices, Sunnyvale, CA). When the calpstatin-TAT (CPS-TAT) inhibitor was used, the cells were incubated with the inhibitor $(2 \mu \mathrm{mol} / \mathrm{L})$ for 1 hour before addition of the substrate and the assay was conducted in the presence of the inhibitor. Fluorescence was assessed using SOFTmax-pro software at an excitation wavelength of $360 \mathrm{~nm}$ and an emission wavelength of $465 \mathrm{~nm}$. Readings were taken every 3 minutes for a period of 45 minutes. Columns in graphs represent maximum relative fluorescence units, whereas error bars are the SD values (of either of the samples with or without CPS-TAT) of two to four duplicate measurements after subtraction of activity obtained in the presence of CPS-TAT. Line graph represents kinetic readings of triplicate measurements with SDs. The lines were curve-fitted after time zero values were normalized.

Method 2. Fluorescence microscopy using $t$-butoxycarbonyl-LeuMet-chloromethylaminocoumarin substrate. Caspase- $8^{-/-}$versus caspase- $8^{+/+}$MEFs were plated on glass-insert $35 \mathrm{~mm}$ dishes (Mattek), serum-starved overnight, and loaded with $10 \mu \mathrm{mol} / \mathrm{L} t$-butoxycarbonylLeu-Met-chloromethylaminocoumarin (Boc-LM-CMAC) for 16 minutes on a heated microscope stage $(47,48)$. EGF $(10 \mathrm{nmol} / \mathrm{L})$ was added and time-lapse photographs were taken every minute for 16 minutes using the $4^{\prime}, 6$-diamidino-2-phenylindole (DAPI) filter for the fluorescent signal and phase contrast. The original raw images had different overall brightness levels, which were normalized using the numerical values of a small sample of background area, generating images that subjectively resembled those observed under the microscope; thus, these images are nonquantitative.

\section{Rac Activity Assays}

Cells were incubated overnight in DMEM with $0.5 \%$ FBS, detached by trypsinization and neutralized with an equal volume of $2.5 \mathrm{mg} / \mathrm{mL}$ soybean trypsin inhibitor, or detached by treatment with TrypLe Express 
(Invitrogen) and neutralized by 10-fold dilution in serum-free medium, followed by centrifugation. The cells were then resuspended in serum-free DMEM containing $20 \mathrm{mg} / \mathrm{mL}$ BSA and plated onto a series of $60 \mathrm{~mm}$ dishes that had been precoated with $20 \mu \mathrm{g} / \mathrm{mL}$ fibronectin (Calbiochem) and blocked with $3 \%$ BSA. At the indicated times, cells were scraped into $0.4 \mathrm{~mL}$ cold lysis buffer $[0.5 \%$ Triton $\mathrm{X}-100,50 \mathrm{mmol} / \mathrm{L}$ Tris $(\mathrm{pH} 7.5), 150$ $\mathrm{mmol} / \mathrm{L} \mathrm{NaCl}, 10 \%$ glycerol, $10 \mathrm{mmol} / \mathrm{L} \mathrm{MgCl}_{2}+1 \times$ complete Roche protease inhibitors, EDTA-free] and precleared. Ten micrograms of bacterially expressed GST-PAK (CRIB domain) protein were added and the samples were incubated on ice for 1 hour, followed by addition of $30 \mu \mathrm{L}$ of a $50 \%$ slurry of glutathione-Sepharose (Pharmacia, Piscataway, NJ; equilibrated in lysis buffer containing $20 \mathrm{mg} / \mathrm{mL}$ BSA) and further incubation for 1 hour in the cold room on a rotation device. Beads were washed thrice in lysis buffer and analyzed on Western blots for Rac using monoclonal antibodies $(\mathrm{mAb})$ from BD PharMingen, peroxidase-labeled antimouse antibody (Invitrogen), and Pierce West Pico chemiluminescence kit (Pierce, Rockford, IL).

\section{Immunofluorescence}

Cells grown on coverslips were fixed with cold methanol for the tubulin/ pericentrin staining or with $4 \%$ paraformaldehyde for the cortactin/actin staining, followed by 5 -minute permeabilization with $0.1 \%$ Triton $\mathrm{X}-100$ for the latter. Cells were stained with anti- $\alpha$-tubulin mouse mAb (DM1A; Calbiochem), antipericentrin polyclonal antibody (Covance, Berkley, CA), anticortactin mAb (provided by Scott Weed) followed by Alexa-488- or Alexa-594-labeled secondary antibodies (Pierce) and mounting with Vectashield containing DAPI (Vector Labs, Burlingame, CA) using AxioVision software and imported into Photoshop, where individual color intensities and contrast were adjusted for clarity of presentation; image intensities are subjectively accurate but nonquantitative.

\section{Results and Discussion}

Caspase-8 regulates cell motility. We assayed MEFs obtained from caspase-8 knockout mice (14) for motility using an ECIS instrument (49). Caspase-8-null cells were motility deficient (Fig. 2A), in agreement with conventional wound healing (Fig. $2 B$ ) and chemotaxis/transwell assays (Fig. $2 C$ ). To exclude the possibility that these MEFs might differ in motility due to caspase-8-independent epiphenomena, we expressed the viral caspase-1/8 inhibitor protein $\mathrm{crmA}(44)$ in the wild-type MEFs and in WTNR6 fibroblasts; crmA inhibited motility efficiently in both (Supplementary Figs. S1 and S2). To further exclude epiphenomena, reexpression of inducible caspase- 8 gene in caspase-8-null MEFs stimulated cell motility (Supplementary Fig. S3). The knockout of the caspase-8 effector, caspase-3, did not suppress cell motility (Supplementary Fig. S4). This result suggests that caspase- 8 may act directly rather than through caspase-3 to cleave substrates critical for cell motility, although other effector caspases cannot be excluded.

Although different cell types undoubtedly use diverse cell motility pathways, similar results were obtained in NB7 neuroblastoma cells (45) and MDA-MB-231 mammary carcinoma cells (Supplementary Fig. S5-S7; note that the effect in NB7 cells was observed in single-cell motility and ECIS assays but not in a standard wound-healing assay, presumably reflecting greater sensitivity of detection and resistance to cell-to-cell interaction effects in the assays used; data not shown). Thus, caspase-8 may promote motility in tumor cells, potentially enhancing tumor metastasis in contexts where cell migration is rate limiting (e.g., ref. 37). In certain specific tumor types, such as neuroblastoma, where caspase- 8 is silenced or deleted, reexpression of caspase8 can, paradoxically, suppress metastasis by conferring anoikis sensitivity (50). However, most metastatic tumor cells express
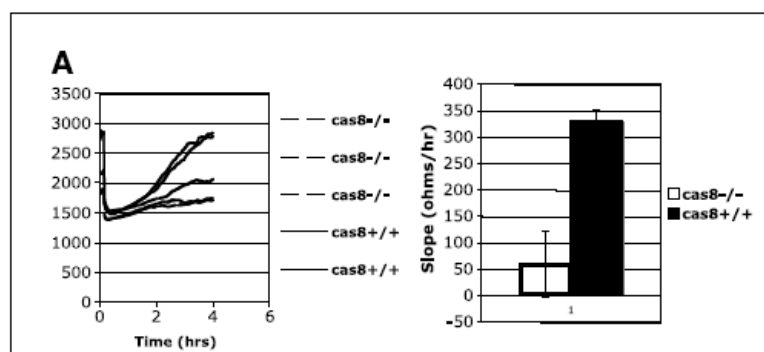

B

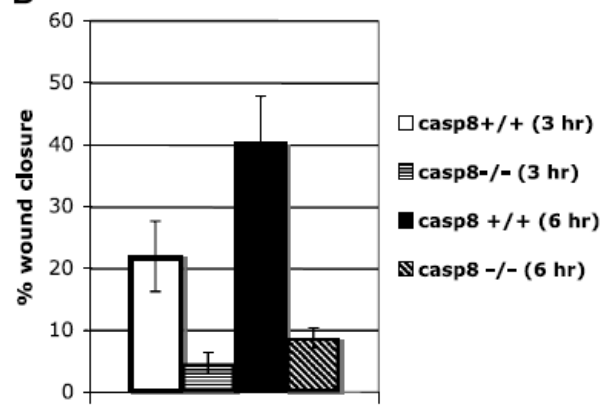

C

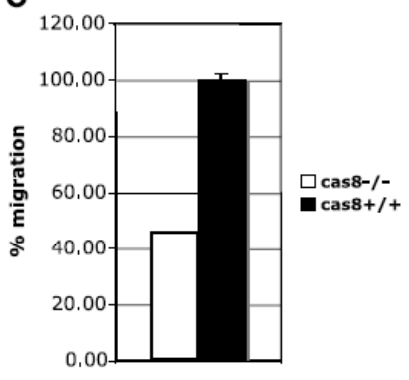

Figure 2. Caspase-8 promotes cell motility. A, ECIS analysis of caspase- $8^{-1}$ versus caspase- $-8^{+/+}$MEFs. Left, impedance versus time plot. Right, slopes calculated from the linear part of the graphs in the ECIS tracing (after subtraction of slopes measured in "unwounded" wells). $B$, conventional wound healing assay, comparing caspase- $8^{-/-}$versus caspase- $8^{+/+} \mathrm{MEFs}$. $C$, directed migration/chemotaxis assay, comparing caspase- $8^{-/-}$versus caspase- $8^{+/+}$ MEFs, normalized for total number of adherent cells.

active caspase- 8 but are, by definition, resistant to anoikis. This resistance is presumably due to alterations in numerous other pathways (e.g., bcl-2 family members, integrin signaling molecules, and autocrine matrix generation). Thus, in most human tumors, the presence or absence of caspase- 8 is not the major parameter of anoikis sensitivity. In summary, although caspase- 8 is metastasis suppressive in neuroblastoma, it is potentially metastasis promoting in other tumor types because it stimulates cell migration.

Caspase-8 promotes calpain activation. Cell motility is dependent on calpain activity in numerous cell systems. In particular, the dependence of cell motility on calpain has been shown in MEFs using genetic knockout of calpain-4 (51) and in WTNR6 cells by using antisense RNA (43).

We tested the effect of caspase- 8 on cell motility in these calpain-dependent cell types. Caspase- 8 promoted calpain activity in both of these cell lines as well as in a tumor cell line (Fig. $3 A-C$; Supplementary Fig. S8). To exclude epiphenomena, inducible 
A

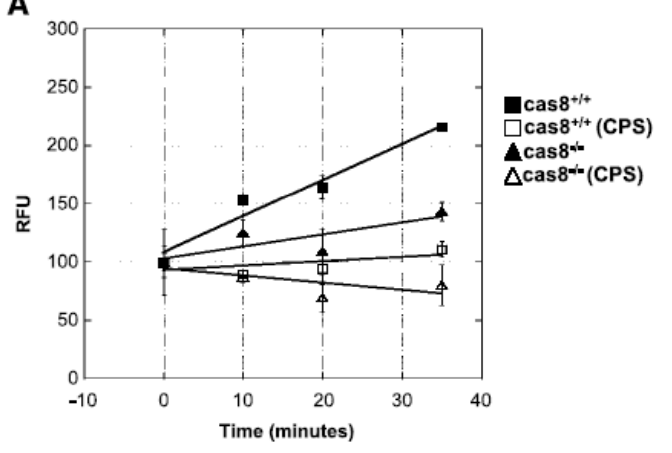

D

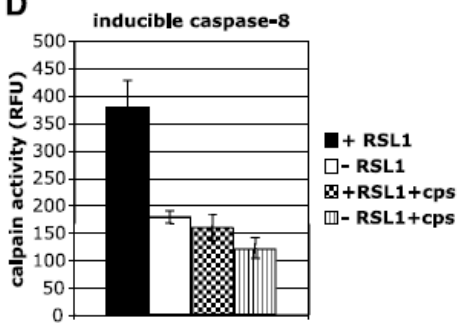

B
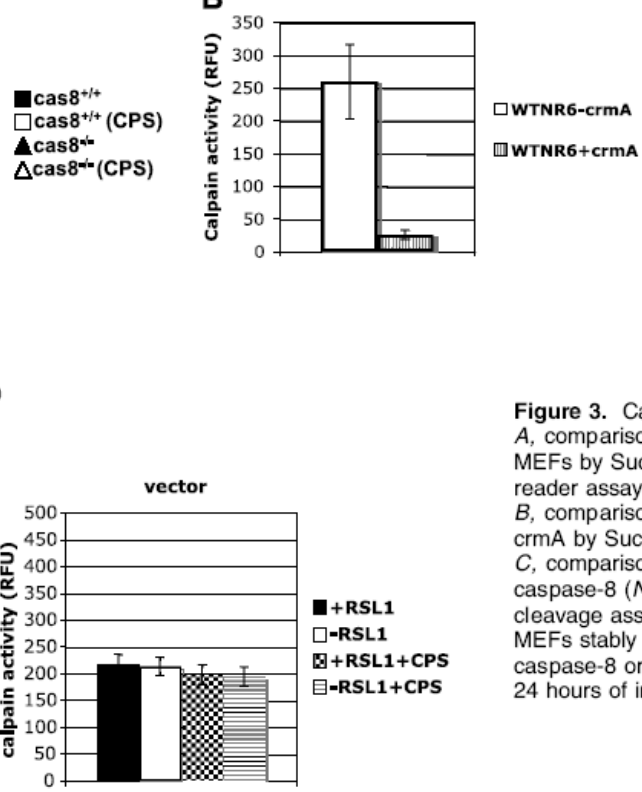

C

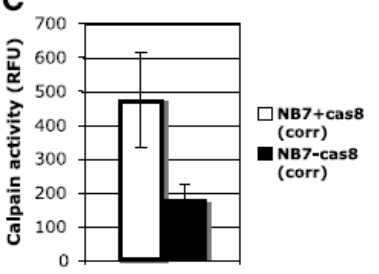

Figure 3. Caspase-8 promotes calpain activity. $A$, comparison of caspase $-8^{-1-}$ versus caspase- $8^{+/+}$ MEFs by Suc-LLVY-AMC cleavage/fluorimetric plate reader assay. $R F U$, relative fluorescence units. $B$, comparison of WTNR6 cells with or without crmA by Suc-LLVY-AMC cleavage assay.

$C$, comparison of NB7 cells with vector alone or with caspase-8 (NB7+cas 8$)$ using the Suc-LLVY-AMC cleavage assay. $D$, comparison of caspase-8-null MEFs stably transfected with Rheoswitch-inducible caspase-8 or with Rheoswitch vector alone after 24 hours of induction.

reexpression of caspase- 8 in caspase-8-null MEFs stimulated calpain activity (Fig. 3D). By contrast, caspase-3 knockout had no significant effect on calpain activity (Supplementary Fig. S9). These data indicate that caspase-8 promotes calpain activity and cell motility in cell types whose motility is calpain dependent. Although these data suggest that caspase- 8 promotes motility through calpain activation, additional effects of caspase8 on calpain-independent aspects of cell motility cannot be excluded.

Calpain activity is often elevated in transformed cells (33) where it promotes cell motility/invasion/metastasis $(35,37)$. Our results suggest that caspase- 8 promotes calpain activity, thereby augmenting these malignant properties of tumor cells.

Caspase-8 affects cellular processes downstream of calpain activation. To confirm that caspase- 8 regulates the calpain pathway, we examined several calpain-dependent processes for caspase-8 dependence: Rac activation, lamellipodial assembly, cell adhesion, and accurate nuclear segregation. First, Rac activation is calpain dependent in certain cell systems (52). Consistent with this, caspase- $8^{-/-}$cells activated Rac inefficiently compared with control cells (Fig. 4). This suggests that caspase- 8 promotes rac activation and subsequent cytoskeletal remodeling through calpains. Second, the genetic knockout of calpain-4 or small interfering RNA (siRNA) depletion of calpain-2 inhibits lamellipodia formation in MEFs (51, 53). Accordingly, caspase-8-deficient MEFs were defective in generating lamellipodia (Fig. $5 A$ ). Interestingly, the caspase-8-null MEFs exhibited numerous long, thin projections resembling calpain-4-knockout MEFs (51) or calpain-2 knockdown cells (53). Conversely, the induced reexpression of caspase- 8 in caspase-8-null MEFs restored their ability to form lamellipodia (Fig. $5 B$ ). Third, calpains are involved in both adhesion complex assembly and disassembly $(52,54)$, suggesting that cell adhesion per se could be affected either positively or negatively in a contextdependent fashion. Caspase-8 promoted cell adhesion in MEFs and
NB7 cells (Fig. 5 C). These results suggested that caspase- 8 also promotes cell adhesion, although cell type specificity of this effect was observed in that caspase-8 reduced cell adhesion in two epithelial cell lines (data not shown).

Fourth, calpain activity is required for accurate alignment of chromosomes on the metaphase plate; siRNA depletion of calpain causes misalignment and increases the frequency with which cytokinesis is aborted, resulting in multinucleate cells (55). Consistent with this, caspase-8-null MEFs displayed an elevated frequency of multinucleate cells (Supplementary Fig. S10). This

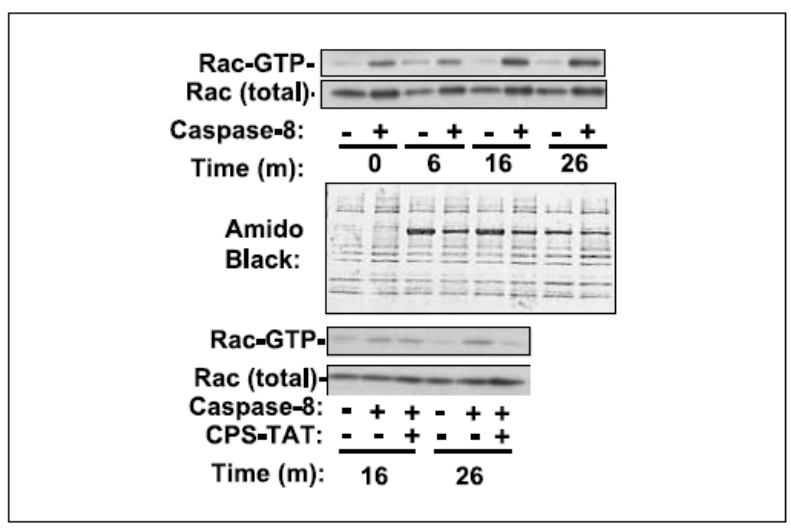

Figure 4. Caspase-8 promotes Rac activity. Caspase- $8^{-1-}$ or caspase- $8^{+/+}$ MEFs were assayed at the indicated times after attachment to fibronectin for activated or total Rac; the Amido Black-stained filter shows equal loading (the BSA band arises from the carrier protein used in the cell attachment incubation buffer; the zero time sample is free of BSA because cells were immediately lysed after trypsinization.) Bottom, a similar experiment was done with or without the addition of CPS-TAT protein to the indicated samples at time zero. (Note that import normally takes 5 to 10 minutes, consistent with the lack of effect at the first time point). 
A

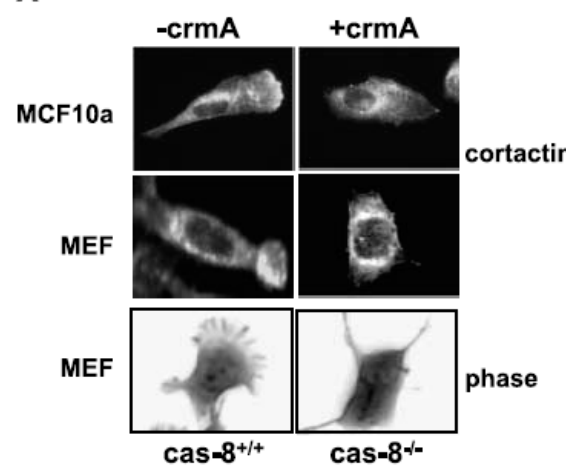

C

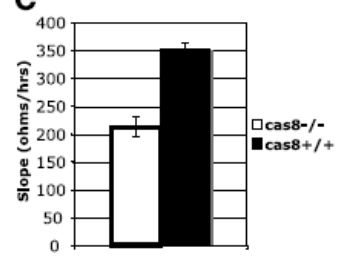

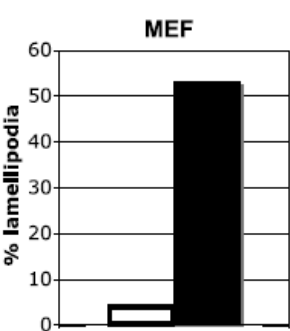
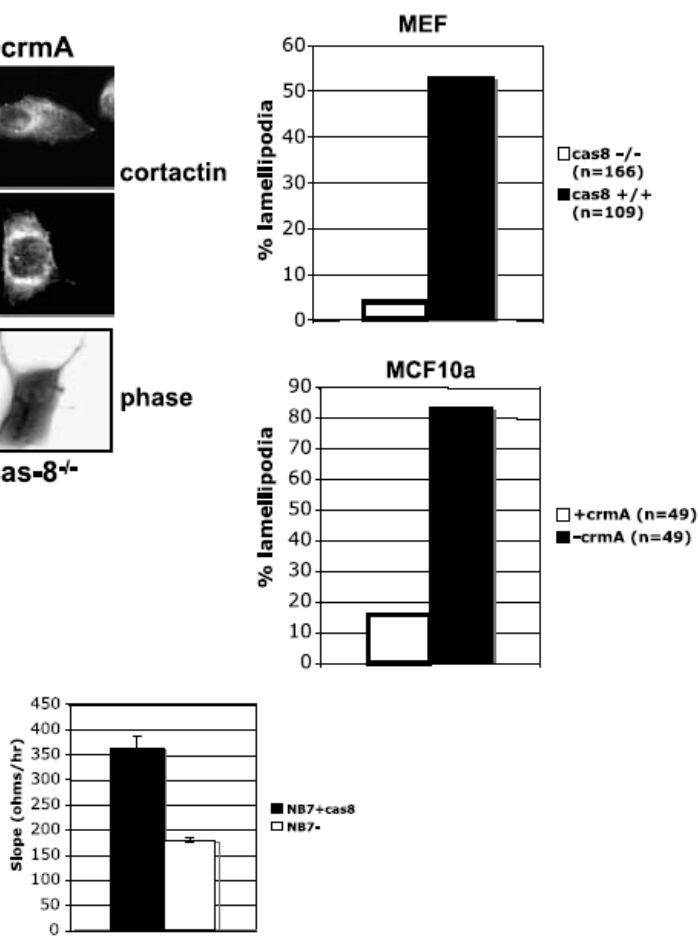

B

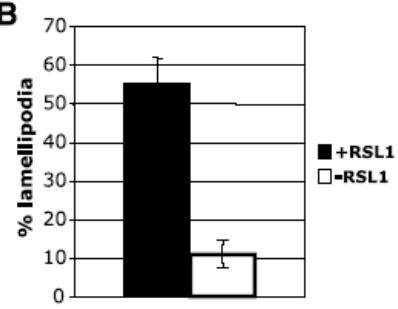

Figure 5. Caspase-8 affects cellular processes downstream of calpain activation: lamellipodial assembly, cell adhesion, and normal nuclear segregation. $A$, caspase-8 promotes the accumulation of lamellipodia. Top and middle, MCF10a cells with or without crmA were plated at low density in medium containing EGF, fixed, and stained with antibodies against the lamellipodia marker cortactin (green), rhodamine-phalloidin (red), and DAPI (blue). Bottom, the "attachment-control" MEFs that were assayed for chemotactic migration in Fig. $1 C$ were stained with crystal violet and photographed. Frequencies of lamellipodia were scored and represented graphically (the phase images were used to generate the MEF graph). MEFs, $P<0.003$; MCF10a, $P<0.001$. B, caspase- 8 promotes the accumulation of lamellipodia: caspase-8-inducible cell lines. $C$, caspase-8 promotes cell adhesion to matrix in MEF cells and NB7 cells. latter result may connect the paradoxical caspase- 8 down-regulation that is observed in restricted tumor types (45) with chromosomal instability (56).

Elucidation of the mechanism by which caspase- 8 activates calpains is in progress. Caspase- 8 could affect the phosphorylation of calpain-2 (48), the levels of the endogenous inhibitor calpastatin (46), or the availability of calcium or phospholipids for interaction with calpains (33); caspase-8 had no effect on the expression of calpain-1 or calpain-2 (data not shown).

The elevated caspase- 8 activities in many tumor cell lines (2) may be causally related to their increased calpain activity, motility, and invasiveness. In this connection, inhibition of calpains suppresses invasion in certain tumor models $(35,37)$, whereas activation of several signaling pathways, including caspase-8, by
FASL promotes invasion (57), suggesting that caspase-8 inhibition may provide the basis for a novel antimetastatic therapy in tumor types that are anoikis-resistant due to other gene alterations.

\section{Acknowledgments}

Received 11/22/2005; revised 2/2/2006; accepted 2/10/2006. Grant support: NIH PO1 grant.

The costs of publication of this article were defrayed in part by the payment of page charges. This article must therefore be hereby marked advertisement in accordance with 18 U.S.C. Section 1734 solely to indicate this fact.

We thank Chris Waters, Fred Minnear, and Charles Keese for support with ECIS; Scott Weed (West Virginia University, Morgantown, WV) for cortactin antibody and helpful discussions; Vipul Shah for technical assistance; Alan Wells (University of Pittsburgh, Pittsburgh, PA) and Richard Flavell (Yale University School of Medicine, New Haven, CT) for cell lines; and Steve Dowdy (University of California, San Diego, La Jolla, CA) for TAT vectors.

\section{References}

1. He L, Wu X, Meylan F, et al. Monitoring caspase activity in living cells using fluorescent proteins and flow cytometry. Am J Pathol 2004;164:1901-13.

2. Yang L, Cao Z, Yan H, Wood WC. Coexistence of high levels of apoptotic signaling and inhibitor of apoptosis proteins in human tumor cells: implication for cancer specific therapy. Cancer Res 2003;63:6815-24.

3. Fulda S, Los M, Friesen C, Debatin KM. Chemosensitivity of solid tumor cells in vitro is related to activation of the CD95 system. Int J Cancer 1998;76:105-14.

4. Owen-Schaub L, Chan H, Cusack JC, Roth J, Hill LL. Fas and Fas ligand interactions in malignant disease. Int J Oncol 2000;17:5-12.

5. Kataoka T. The caspase- 8 modulator c-FLIP. Crit Rev Immunol 2005;25:31-58.
6. Boatright KM, Deis C, Denault JB, Sutherlin DP, Salvesen GS. Activation of caspases- 8 and -10 by FLIP(L). Biochem J 2004;382:651-7.

7. Algeciras-Schimnich A, Barnhart BC, Peter ME Apoptosis-independent functions of killer caspases. Curr Opin Cell Biol 2002;14:721-6.

8. Tibbetts MD, Zheng L, Lenardo MJ. The death effector domain protein family: regulators of cellular homeostasis. Nat Immunol 2003;4:404-9.

9. Wang J, Lenardo MJ. Roles of caspases in apoptosis, development, and cytokine maturation revealed by homozygous gene deficiencies. J Cell Sci 2000;113: $753-7$.

10. Miura M, Chen XD, Allen MR, et al. A crucial role of caspase- 3 in osteogenic differentiation of bone marrow stromal stem cells. J Clin Invest 2004;114:1704-13. 11. Launay S, Hermine O, Fontenay M, Kroemer G, Solary
E, Garrido C. Vital functions for lethal caspases. Oncogene 2005:24:5137-48.

12. Yamaguchi H, Wyckoff J, Condeelis J. Cell migration in tumors. Curr Opin Cell Biol 2005;17:559-64.

13. Geisbrecht ER, Montell DJ. A role for Drosophila IAP1-mediated caspase inhibition in Rac-dependent cell migration. Cell 2004;118:111-25.

14. Varfolomeev EE, Schuchmann M, Luria V, et al Targeted disruption of the mouse caspase 8 gene ablates cell death induction by the TNF receptors, Fas/Apol, and DR3 and is lethal prenatally. Immunity 1998;9:267-76.

15. Kang TB, Ben-Moshe T, Varfolomeev EE, et al. Caspase-8 serves both apoptotic and nonapoptotic roles. J Immunol 2004:173:2976-84

16. van de Water B, Nagelkerke JF, Stevens JL. Dephosphorylation of focal adhesion kinase (FAK) and loss of focal contacts precede caspase-mediated cleavage of 
FAK during apoptosis in renal epithelial cells. J Biol Chem 1999:274:13328-37.

7. Williams ST, Smith AN, Cianci CD, Morrow JS, Brown

TL. Identification of the primary caspase 3 cleavage site in $\alpha$ II-spectrin during apoptosis. Apoptosis 2003;8:353-61.

18. Petrache I, Birukov K, Zaiman AL, et al. Caspasedependent cleavage of myosin light chain kinase (MLCK) is involved in TNF- $\alpha$-mediated bovine pulmonary endothelial cell apoptosis. FASEB J 2003;17:407-16. 19. Stegh AH, Herrmann H, Lampel S, et al. Identification of the cytolinker plectin as a major early in vivo substrate for caspase 8 during $\mathrm{CD}^{-}$and tumor necrosis factor receptor-mediated apoptosis. Mol Cell Biol 2000;20:5665-79.

20. Widmann C, Gerwins P, Johnson NL, Jarpe MB Johnson GL. MEK kinase 1, a substrate for DEVDdirected caspases, is involved in genotoxin-induced apoptosis. Mol Cell Biol 1998:18:2416-29.

21. Cardone MH, Salvesen GS, Widmann C, Johnson G, Frisch SM. The regulation of anoikis: MEKK-1 activation requires cleavage by caspases. Cell 1997;90:315-23.

22. Deak JC, Cross JV, Lewis M, et al. Fas-induced proteolytic activation and intracellular redistribution of the stress-signaling kinase MEKK1. Proc Natl Acad Sci U S A 1998;95:5595-600.

23. Na S, Chuang TH, Cunningham A, et al. D4-GDI, a substrate of CPP32, is proteolyzed during Fas-induced apoptosis. J Biol Chem 1996:271:11209-13.

24. Krieser RJ, Eastman A. Cleavage and nuclear translocation of the caspase 3 substrate Rho GDP dissociation inhibitor, D4-GDI, during apoptosis. Cell Death Differ 1999;6:412-9.

25. Kato M, Nonaka T, Maki M, Kikuchi H, Imajoh-Ohmi S. Caspases cleave the amino-terminal calpain inhibitory unit of calpastatin during apoptosis in human Jurkat T cells. J Biochem (Tokyo) 2000;127:297-305.

26. Porn-Ares MI, Samali A, Orrenius S. Cleavage of the calpain inhibitor, calpastatin, during apoptosis. Cell Death Differ 1998;5:1028-33.

27. Wang KK, Posmantur R, Nadimpalli R, et al. Caspasemediated fragmentation of calpain inhibitor protein calpastatin during apoptosis. Arch Biochem Biophys 1998;356:187-96

28. Neumar RW, Xu YA, Gada H, Guttmann RP, Siman R Cross-talk between calpain and caspase proteolytic systems during neuronal apoptosis. J Biol Chem 2003:278:14162-7.

29. Zhang B, Zhang Y, Shacter E. Caspase 3-mediated inactivation of rac GTPases promotes drug-induced apoptosis in human lymphoma cells. Mol Cell Bio 2003;23:5716-25.
30. Tu S, Cerione RA. Cdc42 is a substrate for caspase and influences Fas-induced apoptosis. J Biol Chem 2001;276:19656-63.

31. Sebbagh M, Renvoize C, Hamelin J, Riche N, Bertoglio J, Breard J. Caspase-3-mediated cleavage of ROCK induces MLC phosphorylation and apoptotic membrane blebbing. Nat Cell Biol 2001;3:346-52.

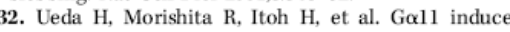
caspase-mediated proteolytic activation of Rho-associated kinase, ROCK-I, in HeLa cells. J Biol Chem 2001:276:42527-33.

33. Franco SJ, Huttenlocher A. Regulating cell migration: calpains make the cut. J Cell Sci 2005;118:3829-38. 34. Arthur JS, Elce JS, Hegadorn C, Williams K, Greer PA Disruption of the murine calpain small subunit gene, Capn4 calpain is essential for embryonic development but not for cell growth and division. Mol Cell Biol 2000;20:4474-81. 35. Kassis J, Radinsky R, Wells A. Motility is rate-limiting for invasion of bladder carcinoma cell lines. Int Biochem Cell Biol 2002;34:762-75.

36. Carragher NO, Fonseca BD, Frame MC. Calpain activity is generally elevated during transformation but has oncogene-specific biological functions. Neoplasia 2004;6:53-73.

37. Mamoune A, Luo JH, Lauffenburger DA, Wells A Calpain-2 as a target for limiting prostate cance invasion. Cancer Res 2003;63:4632-40.

38. Braun C, Engel M, Seifert M, et al. Expression of calpain I messenger RNA in human renal cell carcinoma: correlation with lymph node metastasis and histological type. Int J Cancer 1999;84:6-9.

39. Rios-Doria J, Kuefer R, Ethier SP, Day ML. Cleavage of $\beta$-catenin by calpain in prostate and mammary tumo cells. Cancer Res 2004:64:7237-40.

40. Rios-Doria J, Day KC, Kuefer R, et al. The role of calpain in the proteolytic cleavage of E-cadherin in prostate and mammary epithelial cells. J Biol Chem 2003;278:1372-9.

11. Wang Y, Klijn JG, Zhang Y, et al. Gene-expression profiles to predict distant metastasis of lymph-nodenegative primary breast cancer. Lancet 2005;365:671-9. 42. Zheng TS, Hunot S, Kuida $K$, et al. Deficiency in caspase-9 or caspase- 3 induces compensatory caspase activation. Nat Med 2000;6:1241-7.

43. Glading A, Chang P, Lauffenburger DA, Wells A. Epidermal growth factor receptor activation of calpain is required for fibroblast motility and occurs via an ERK/MAP kinase signaling pathway. J Biol Chem 2000;275:2390-8.

4. Zhou Q, Snipas S, Orth K, Muzio M, Dixit VM, Salvesen GS. Target protease specificity of the vira serpin CrmA. Analysis of five caspases. J Biol Chem 1997:272:7797-800

5. Teitz T, Wei T, Valentine MB, et al. Caspase 8 is deleted or silenced preferentially in childhood neuroblastomas with amplification of MYCN. Nat Med 2000;6:529-35.

16. Wendt A, Thompson VF, Goll DE. Interaction of calpastatin with calpain: a review. Biol Chem 2004 385:465-72.

47. Cuevas BD, Abell AN, Witowsky JA, et al. MEKK regulates calpain-dependent proteolysis of focal adhesion proteins for rear-end detachment of migrating fibroblasts. EMBO J 2003:22:3346-55.

8. Glading A, Bodnar RJ, Reynolds IJ, et al. Epidermal growth factor activates m-calpain (calpain II), at least in part, by extracellular signal-regulated kinasemediated phosphorylation. Mol Cell Biol 2004:24: 2499-512.

9. Keese CR, Wegener J, Walker SR, Giaever I. Electrica wound-healing assay for cells in vitro. Proc Natl Acad Sci U S A 2004;101:1554-9.

50. Stupack DG, Teitz T, Potter MD, et al. Potentiation of neuroblastoma metastasis by loss of caspase- 8 . Nature 2006;439:95-9.

1. Dourdin N, Bhatt AK, Dutt P, et al. Reduced cel migration and disruption of the actin cytoskeleton in calpain-deficient embryonic fibroblasts. J Biol Chem 2001;276:48382-8.

2. Bialkowska K, Saido TC Fox JE SH3 domain of spectrin participates in the activation of Rac in specialized calpain-induced integrin signaling complexes. J Cell Sci 2005;118:381-95.

53. Franco S, Perrin B, Huttenlocher A. Isoform specific function of calpain 2 in regulating membrane protrusion. Exp Cell Res 2004:299:179-87.

54. Carragher NO, Westhoff MA, Fincham VJ, Schalle MD, Frame MC. A novel role for FAK as a protease targeting adaptor protein: regulation by $\mathrm{p} 42$ ERK and Src. Curr Biol 2003;13:1442-50.

55. Honda S, Marumoto T, Hirota T, et al. Activation of $\mathrm{m}$-calpain is required for chromosome alignment on the metaphase plate during mitosis. J Biol Chem 2004;279. 10615-23.

56. Fujiwara T, Bandi M, Nitta M, Ivanova EV, Bronson RT, Pellman D. Cytokinesis failure generating tetraploids promotes tumorigenesis in p53-null cells. Nature 2005 437:1043-7.

7. Barnhart BC, Legembre P, Pietras E, Bubici C Franzoso G, Peter ME. CD95 ligand induces motility and invasiveness of apoptosis-resistant tumor cells. EMBO J 2004:23:3175-85. 


\title{
Caspase-8 Interacts with the p85 Subunit of Phosphatidylinositol 3-Kinase to Regulate Cell Adhesion and Motility
}

\author{
Jamie Senft, Brooke Helfer, and Steven M. Frisch \\ Mary Babb Randolph Cancer Center, West Virginia University, Morgantown, West Virginia
}

\begin{abstract}
Cell migration plays an important role in tumor cell invasion and metastasis. Previously, we reported that caspase- 8 contributes to cell migration and adhesion, a novel nonapoptotic function of an established apoptotic factor. Herein, we report that pro-caspase- 8 is capable of restoring cell migration/ adhesion to caspase-8-null cells, establishing the first biological function of a pro-caspase. The catalytic activity of caspase-8 was not required for cell motility. Stimulation of motility with epidermal growth factor induced the phosphorylation of caspase- 8 on tyrosine-380 and the interaction of caspase- 8 with the $p 85 \alpha$ subunit of phosphatidylinositol 3-kinase. Tyrosine-380 was required for the restoration of cell motility and cell adhesion in caspase-8-null cells, demonstrating the importance of the caspase-8-p85 interaction for these nonapoptotic functions. These results suggest that caspase8 phosphorylation converts it from a proapoptotic factor to a cell motility factor that, through tyrosine-380, interacts with p85, an established cell migration component. [Cancer Res 2007;67(24):11505-9]
\end{abstract}

\section{Introduction}

Cell migration plays a critical role in tumor cell invasion and metastasis (1). Previously, we reported that caspase-8 promotes cell migration, cell adhesion, and Rac activation in normal and tumor cell lines (2). Caspase- 8 has also been implicated in the proinvasive effects of FASL in tumor cells (3). Although the genesis of certain tumor types such as neuroblastoma and small-cell lung cancer involves the loss of caspase- 8 expression, which suppresses anoikis in neuroblastomas (4), expression is maintained or increased in most tumor types (Supplementary Fig. S1). Thus, caspase-8 may contribute to metastasis in the more typical anoikis-resistant tumor cell context. This mechanism could have important ramifications for cancer therapy.

Phosphatidylinositol 3-kinase (PI3K) is an important component of the cell migration apparatus. PI3K supports Rac/cdc42 activation both by production of lipid products that activate or localize guanine exchange factors (GEF; ref. 5), as well as through direct interactions of Rac, cdc42, and Rac-GEFs with the p85 regulatory subunit $(6,7)$, which are involved in cdc42mediated c-Jun- $\mathrm{NH}_{2}$-kinase activation (8). P85 function is affected by the binding of tyrosine phosphorylated proteins to

Note: Supplementary data for this article are available at Cancer Research Online (http://cancerres.aacrjournals.org/).

J. Senft and B. Helfer are co-first authors.

Requests for reprints: Steven M. Frisch, Mary Babb Randolph Cancer Center, West Virginia University, 1 Medical Center Drive, Campus Box 9300, Morgantown, WV

26506. Phone: 304-293-2980; Fax: 304-293-4667; E-mail: sfrisch@hsc.wvu.edu.

(C2007 American Association for Cancer Research.

doi:10.1158/0008-5472.CAN-07-5755 its SH2 domains, as well as src-mediated phosphorylation (9). The potential for caspases to interact functionally with PI3K has not been explored.

In apoptotic cells, caspase- 8 undergoes two sequential autoproteolytic cleavage reactions that separate the large and small catalytic subunits as well as deleting a small linker peptide sequence (amino acids 374-384) and the prodomain (amino acids 1-216), releasing the mature tetramer form into the cytoplasm (10). However, the pro-caspase- 8 form predominates in nonapoptotic cells. In principle, pro-caspase- 8 versus processed caspase- 8 could function as a migration/adhesion and apoptosis factors, respectively. In that scenario, pro-caspase- 8 could potentially cleave key substrates for motility-remaining to be identified-or could function noncatalytically as an interaction partner for a motility factor. In the latter connection, it has been reported recently that pro-caspase- 8 is phosphorylated on tyrosine- 380 by c-src; this phosphorylation suppresses caspase- 8 autocleavage and apoptosis (11). In principle, it could also facilitate the interactions of caspase-8 promigratory proteins containing phosphotyrosine binding domains.

In this report, we show that Y380-phosphorylated pro-caspase-8 interacts with the p85 subunit of PI3K, promoting cell adhesion and motility.

\section{Materials and Methods}

Cell lines. NB7 cells were obtained and cultured as described previously (2). Stable derivatives expressing caspase- 8 mutants were constructed by packaging the appropriate retrovirus construct in the vector pBABE-puro in gp $2+293$ cells in the presence of pAmpho (Invitrogen), infection of viral stock, selection with $0.5 \mu \mathrm{g} / \mathrm{mL}$ puromycin, and Western blotting of mixed populations; similar expression levels were obtained for all constructs used in this study (data not shown). A431 epidermal carcinoma cells were obtained from American Type Culture Collection and maintained in DMEM containing $10 \%$ fetal bovine serum, penicillin, streptomycin, and glutamine.

DNA constructs, transfections, and pull downs. Details of the construction of our expression constructs are provided in the Supplementary Materials and Methods section. For pull-down experiments on transiently transfected S-tagged constructs, cell lysates were incubated with S-protein agarose, washed, and analyzed as described in Supplementary Materials and Methods.

Endogenous coimmunoprecipitation. A431 cells treated with epidermal growth factor (EGF) were lysed and immunoprecipitated with p85 antibodies as described in Supplementary Materials and Methods.

Rac activation assay. Rac activity was assayed by incubation of cell lysates with glutathione $S$-transferase-PAK (CRIB domain) constructs as described in Supplementary Materials and Methods.

SH2 domain array screening. A peptide spanning human caspase8 Y380 [(biotin-K)GGGDSEEQP(phospho-Y)LEMDLSSPQT] was synthesized (Bachem) and reacted with an $\mathrm{SH} 2$ domain array (Panomics), detecting with streptavidin-horseradish peroxidase, according to the manufacturer's protocol, except that detection was with enhanced chemiluminescence reagent (Pierce). 
A

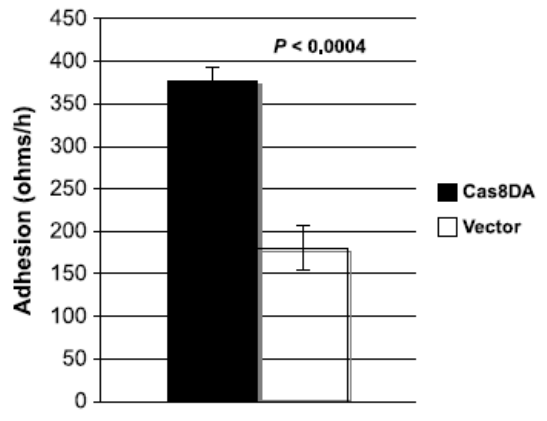

C

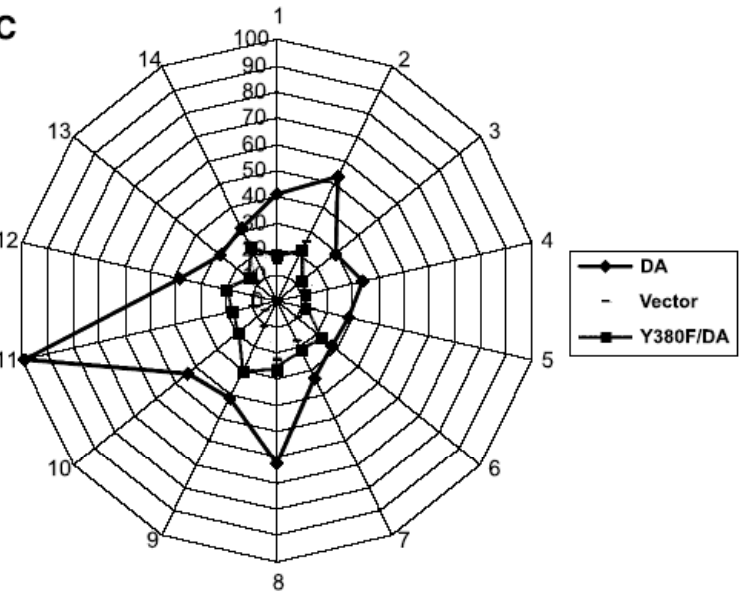

B

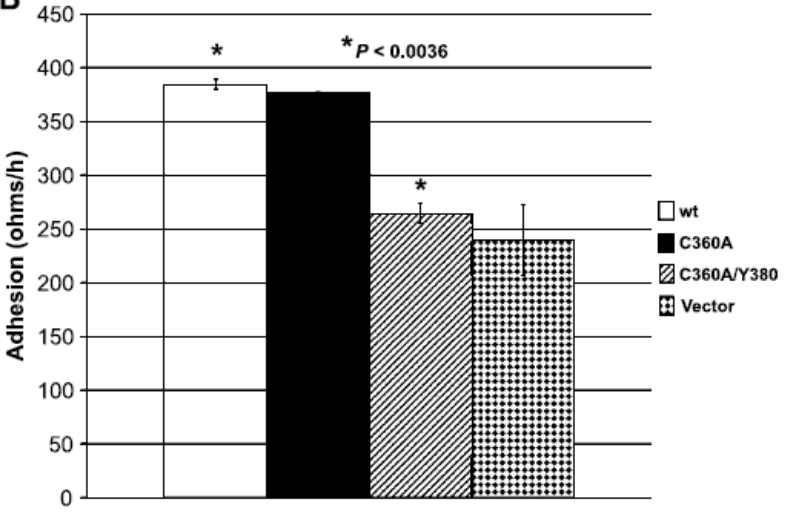

D

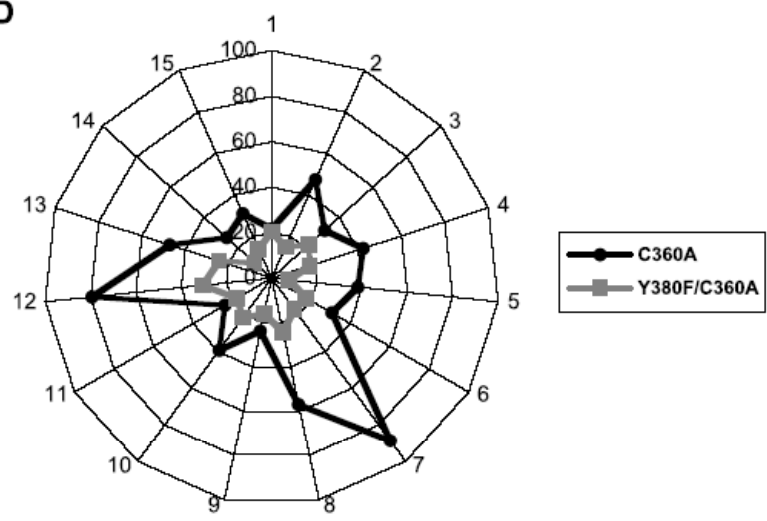

Figure 1. Caspase-8 enhancement of cell adhesion/motility is dependent on tyrosine-380, but independent of catalytic activity or autoproteolytic processing For these experiments, the caspase-8-null cell line NB7 was infected with retroviral caspase-8 expression constructs as indicated and assayed for motility or adhesion. A, pro-caspase-8 rescues adhesion. Cells were assayed for kinetics of adhesion to fibronectin using ECIS; the slopes of the linear portion of the ECIS tracing are plotted; note that the fold stimulation of adhesion is similar to that of wild-type caspase- 8 reported previously (2). Cas $8 D A$, the uncleavable caspase-8 mutant, D374A/D384A; vector, pBABE-puro. B, the catalytic activity of caspase-8 is not required and the phosphorylation site Y380 is critical for cell adhesion. An ECIS adhesion assay comparing the wild-type (wt), catalytically inactive $(C 360 A)$, or the phosphorylation site-defective mutant of inactive $(Y 380 F / C 360 A)$ caspase-8 is shown. $P$ values for the difference between the indicated samples are shown. $C$, pro-caspase-8 promotes cell motility, which is dependent on $Y 380$. A single cell-motility assay is presented as a radial plot indicating distance (not direction) of migration. $D A$, the uncleavable mutant D374A/D384A; Y380F/DA, the phosphorylation site-defective/uncleavable double mutant; vector, pBABE-puro. D, inactive caspase-8 promotes cell motility, which is dependent on Y380. C360A, catalytically inactive caspase-8; Y380F/C360A, the phosphorylation site-defective/catalytically inactive double mutant caspase-8.

Adhesion and motility assays. Adhesion measurements using electrical cell substrate impedance sensing (ECIS) and motility measurements using single-cell motility were done as described previously (2).

Akt phosphorylation assays. Akt phosphorylation after EGF stimulation was monitored on Western blots using a phospho-Akt-specific antibody as described in Supplementary Materials and Methods.

\section{Results and Discussion}

Caspase-8 enhancement of cell adhesion/motility is dependent on tyrosine-380, but independent of catalytic activity or autoproteolytic processing. We reported previously that caspase-8 enhanced EGF-activated cell motility and cellmatrix adhesion in embryonic and adult fibroblasts, as well as several mammary and lung tumor lines, demonstrating the effect of caspase- 8 in diverse cell contexts (2); to facilitate the analysis of caspase-8 mutants, the caspase-8-null NB7 neuroblastoma cell line was used primarily in the current study. The cleavage of caspase-8 was not observed in cells undergoing stimulated motility or cell adhesion (data not shown). We assayed an uncleavable caspase-8 mutant that persists in the full-length procaspase form even in death ligand-stimulated cells and therefore fails to support apoptosis, D374A/D384A (10), for its effects on cell adhesion and motility. When expressed in the caspase-8-null neuroblastoma cell line NB7, this mutant restored both cell adhesion kinetics and single-cell random motility as efficiently as wild-type caspase-8 [compare Fig. 1 with (Supplementary Fig. S6 of ref. 2)]. This result suggested that the pro-caspase- 8 protein, the predominant species observed in nonapoptotic cells, 
functions as a cytoskeletal control element that affects motility and adhesion. Moreover, the inactivation of caspase- 8 catalytic activity (C360A) had no substantial effect on either its motility or adhesion effects (Fig. 1), suggesting a novel noncatalytic mechanism.

Pro-caspase-8 is phosphorylated in cells with hyperactive c-src or stimulated with EGF (11), conditions that stimulate cell motility. The phosphorylation site, Y380, is uniquely present in pro-caspase-8 but absent in processed caspase-8 products. In light of the function of pro-caspase-8 shown above, we hypothesized that this phosphorylation promoted a protein-protein interaction that contributed to the cytoskeletal effects of caspase- 8 . To test this hypothesis, a Y380F (phosphorylation site-deficient) mutant was expressed in NB7 cells in the context of uncleavable (D374A/D384A) or catalytically inactive (C360A) forms of caspase-8. These mutants were defective in rescuing both cell motility and cell adhesion (Fig. 1). This result shows that Y380 plays a substantial role in these nonapoptotic functions of caspase-8. This effect of the phosphorylation site Y380 is clearly distinct from its role in suppressing caspase- 8 processing in that the point mutant Y380F exhibited a strong effect in a caspase-8 mutant that was uncleavable. These results suggested that the phosphorylation at Y380 promoted a

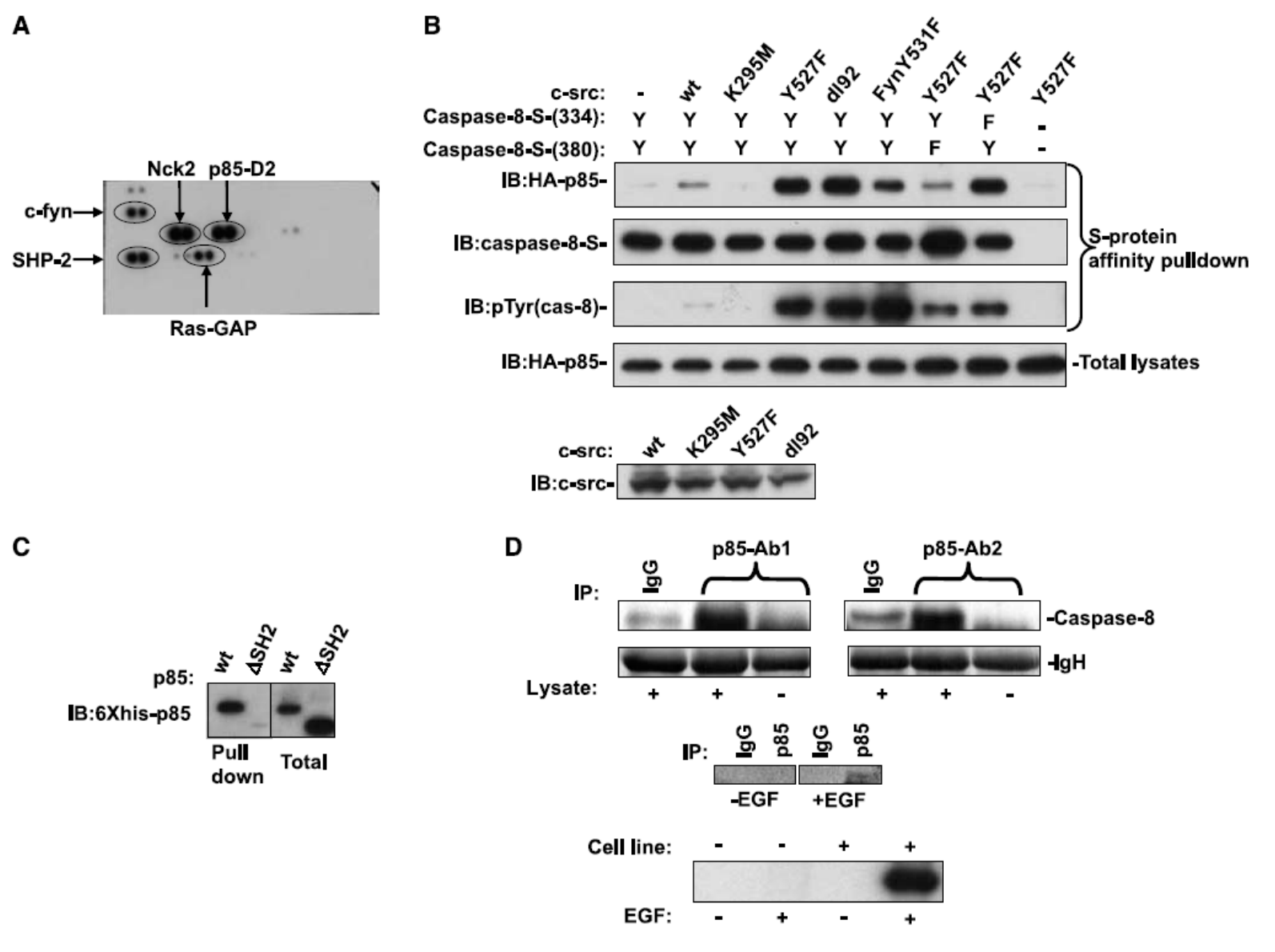

Figure 2. Caspase-8 interacts with p85. $A$, a phosphorylated peptide spanning caspase- 8 Y 380 reveals candidate interaction partners including p85 $B$, caspase-8 interaction with p85: dependence on src and $\mathrm{Y} 380$. $\mathrm{COOH}$-terminally S-tagged constructs of catalytically inactive caspase-8 containing no other mutations $(w t)$, or the mutations $\mathrm{Y} 380 \mathrm{~F}$ or $\mathrm{Y} 334 \mathrm{~F}$ as indicated were cotransfected with the indicated c-src constructs (K295M, catalytically inactive src; Y527F and d/92, activated mutant src) and HA-tagged p85. Lysates were precipitated with S-protein agarose and probed for HA-p85, caspase-8-S-tag, or phosphotyrosine (the band corresponding to caspase-8 is shown). To confirm similar c-src expression levels for the four constructs used, separate wells were transfected, thus avoiding artifactual spillover bands due to the comigration of caspase-8 with c-src (data not shown). IB, immunoblotting. $C$, the $\mathrm{COOH}$-terminal $\mathrm{SH} 2$ domain of p85 is required for the caspase-8 interaction. Caspase-8-S-tag was cotransfected with p85-6Xhis (wild-type or $\Delta \mathrm{SH} 2-\mathrm{COOH}$ terminal) and S-protein agarose pull downs were analyzed for p85 as above. D, endogenous caspase-8-p85 interaction. Top, lysates from EGF-treated A431 cells were immunoprecipitated (IP) with antibodies against p85 $\alpha$, p85-Ab1 (Upstate Biotechnology), or p85-Ab2 (Upstate Biotechnology) or an equal amount of control rabbit IgG. Immunoprecipitates were probed for caspase-8; the Amido Black-stained bands beneath the Western blot show that equal amounts of p85 versus control IgG precipitated. Note that a diffuse band of immunoglobulin heavy chain (not shown) migrated only slightly faster than the caspase-8 band, causing the smear of black density seen at the lower portion of each caspase-8 band. Middle, A431 cells were serum starved, and either induced with EGF (4 min) or uninduced, before the immunoprecipitation with p85-Ab1. For clarity (i.e., to avoid spillover signal from the IgH chain band), a light exposure is shown. Bottom, EGF stimulates caspase-8 phosphorylation. A cell line that expresses S-tagged caspase- 8 at a total caspase- 8 level of $\sim 150 \%$ of the endogenous level was generated (Materials and Methods). This cell line, or, as a control, normal A431 cells were serum starved and treated with EGF $(5 \mathrm{~min})$. Cell lysates were affinity-purified with S-protein agarose and the phosphorylated caspase-8 band was detected on a Western blot by probing with phosphotyrosine antibody. 

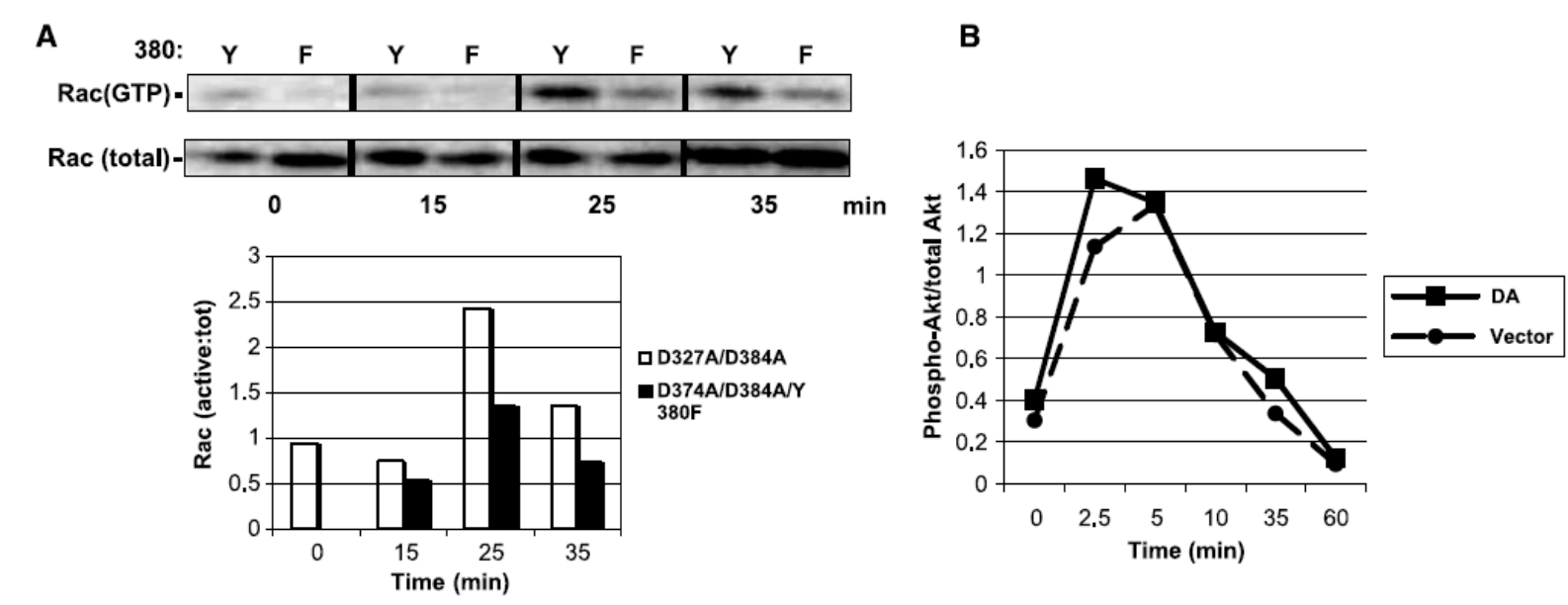

Figure 3. Caspase-8-p85 interaction contributes to Rac activation but does not enhance Akt activation. A, caspase-8-p85 interaction contributes to Rac activation. NB7 cells rescued with uncleavable caspase-8 containing Y380 $(Y)$ or F380 $(F)$ were trypsinized, attached to fibronectin-coated dishes for the indicated times, and lysates were assayed for total or activated Rac. Bottom, a plot of activated Rac to total Rac ratios derived by densitometry; an additional experiment produced similar results. The average fold increase of $\mathrm{Y} 380$ versus F380 at 25 min was $1.67 \pm 0.14$. D374A/D384A, uncleavable caspase-8 mutant; D374A/D384A/Y380F, uncleavable/phosphorylation site-defective caspase-8 mutant. $B$, caspase-8 does not contribute to Akt phosphorylation. NB7 cells with the indicated caspase8 constructs were senum-starved, stimulated with EGF, and assayed for Akt serine-473 phosphorylation by Western blotting. Densitometry of phospho-Akt and total Akt were used to derive ratios, which were plotted here; a lack of effect of caspase-8 on Akt phosphorylation was also observed with matrix-reattachment and serum re-addition (data not shown).

protein interaction that was involved in cell adhesion and/or motility.

Caspase-8 interacts with the p85 subunit of PI3K. Y380 occurs in the peptide sequence YELM, which was predicted by Scansite ${ }^{1}$ to interact with $\mathrm{p} 85 \mathrm{SH} 2$ domains with $99.5 \%$ probability. To test potential interactions with $\mathrm{SH} 2$ domain proteins in an unbiased manner, a SH2 domain array (Panomics) was screened with a phosphorylated, biotinylated peptide spanning this sequence. The array identified the $\mathrm{COOH}$-terminal $\mathrm{SH} 2$ domain of p85, the adaptor protein Nck-2, c-fyn, SHP-2, and Ras-GAP as potential interactors (Fig. $2 A$ ).

In light of the Scansite prediction and these data, we tested the potential of these proteins to interact with an inactive mutant of caspase-8-to prevent apoptosis induction-in transfected cells. P85 strongly interacted with caspase-8 (Fig. $2 B$ ). This interaction depended on the integrity of the Y380 site but was independent of another phosphorylation site, Y334, identified independently by proteomics (12). The interaction was also dependent on cotransfected, activated c-src (Y527F or $\Delta 92-95)$ or c-fyn (Y531F), which were more effective than wild-type c-src or kinase-inactive c-src (K295M). The phosphorylation of caspase- 8 by these src constructs correlated with the magnitude of the caspase-8-p85 interaction. Deletion of the COOH-terminal SH2 domain of p85 abolished the interaction (Fig. $2 \mathrm{C}$ ), implicating this $\mathrm{SH} 2$ domain in the caspase8 interaction. Other candidates identified from the Panomics screen (Nck-2, c-fyn, SHP-2) interacted more weakly or independently of phosphorylation (Supplementary Fig. S2). These data map the interaction on p85 to the $\mathrm{COOH}$-terminal SH2 domain and on caspase-8 to Y380.

To test whether caspase-8 interacted endogenously with p85, total protein from EGF-treated A431 cells was immunopreci-

\footnotetext{
${ }^{1}$ http://scansitemit.edu
}

pitated with polyclonal p85 $\alpha$ antibodies directed against the $\mathrm{NH}_{2}-$ terminal SH2 domain or the full-length protein, followed by Western blotting for caspase- 8 using a highly specific monoclonal antibody. Caspase-8 coimmunoprecipitated with p 85 (Fig. 2D). The reverse coimmunoprecipitation of $\mathrm{p} 85$ with caspase- 8 was observed as well, but a variable background of p85 binding to protein-A beads even under stringent detergent conditions in the presence of protein blocking agents made this direction less reliable (data not shown). These data indicated that caspase8 interacts with p85 $\alpha$ in EGF-stimulated cells. The endogenous caspase-8-p85 interaction was stimulated by EGF, which correlated with the induction of caspase-8 phosphorylation (Fig. $2 D$ ).

The caspase-8-p85 interaction affects Rac activation. Caspase- 8 stimulated the activation of Rac in response to matrix reattachment of mouse embryo fibroblasts (2). In light of the important role of p85 in Rac activation, we hypothesized that the effect of caspase- 8 on Rac was mediated by $p 85$. To test this, NB7 cells with Y380 (wild-type) or F380 (nontyrosine-phosphorylated) forms of caspase- 8 were assayed for Rac activation after reattachment to fibronectin. Consistent with the effect of the Y380F mutation on cell adhesion and motility, this mutation also suppressed Rac activation relative to wild-type caspase-8 (Fig. $3 A$ ). One potential mechanism for the activation of Rac through p85 is the stimulation of PI3K activity, generating lipid products that activate Rac-GEFs. Phosphorylation of Akt on serine473, an indicator of PI3K activity, was not, however, affected by caspase-8, after EGF stimulation (Fig. $3 B$ ) or serum re-addition (data not shown). It is therefore more likely that caspase- 8 affects Rac activation mediated by $\mathrm{p} 85$ through an alternative mechanism that remains to be elucidated.

In principle, caspase- 8 could affect motility and adhesion through multiple mechanisms that may or may not depend on its catalytic activity. Pro-caspase- 8 can acquire catalytic activity by homodimerization mediated by FADD protein or by 
heterodimerization with c-FLIP, and, in apoptotic cells, this proteolytic activity autocatalytically releases the active subunit tetramer form of caspase- 8 into the cytoplasm $(10,13)$. A biological function for pro-caspase-8 other than serving as a precursor for mature caspase- 8 has not yet been found. In this report, we show that neither caspase- 8 processing nor catalytic activity is required for the nonapoptotic cell motility and matrix adhesion functions of caspase- 8 . This dissociates the apoptotic from the nonapoptotic functions of caspase- 8 , which are performed by the processed versus unprocessed forms of the protein, respectively. The observation that Y380 phosphorylation of caspase- 8 by c-src interferes with processing (11), integrated with our current data, suggests a new mechanism for c-src to promote cell migration.

The interaction of phosphorylated caspase- 8 with p 85 and the importance of this interaction for cell motility, cell adhesion, and Rac activation provides one mechanism underlying the effects of caspase- 8 on these processes. It is important to note, however, that additional mechanisms undoubtedly exist, as mouse caspase-8, which lacks a Y380 homologue, can also stimulate cell motility in certain mouse cell lines (2). Although the mouse homologue would not be predicted to be effective in the human cell system used here, there may be other human cell contexts in which other mechanisms involving catalytic activity play a more significant role; in this connection, mouse and human caspase- 8 also enhanced EGF-mediated extracellular signal-regulated kinase activation (data not shown).
Caspase- 8 could regulate PI3K-mediated cell migration (14-16) through at least two pathways. First, caspase-8 could affect PI3K catalytic activity, which generates lipid products (PIP2 and PIP3) that activate GEFs (5). Second, caspase- 8 could affect p85 Bcr-homology domain interaction with Rac/cdc42, and/or the $\mathrm{NH}_{2}$-terminal SH2 domain interaction with a Rac-GEF complex $(6,7,15,17)$. Although the data do not exclude the possibility that caspase- 8 mediates a localized PI3K activation, which could be detected by other experimental approaches, the lack of effect of caspase- 8 on Akt phosphorylation supports the second mechanism. The details of this mechanism are being elucidated currently, as is the possibility that p 85 serves to recruit caspase- 8 to EGF receptor complexes, affecting signaling through this receptor.

Consistent with our observation that caspase- 8 is usually maintained in human tumors, a six-nucleotide polymorphic variant in the caspase- 8 promoter region that attenuates transcription is associated with lowered cancer incidence (18). The contribution of caspase- 8 to cell migration through $p 85$ suggests that caspase- 8 may contribute to tumor progression in this manner.

\section{Acknowledgments}

Received 11/9/2007; revised 11/9/2007; accepted 11/9/2007.

Grant support: Partial support to S.M. Frisch was from the administrative core component of an NIH COBRE grant (D. Flynn, P.I.).

The costs of publication of this article were defrayed in part by the payment of page charges. This article must therefore be hereby marked advertisement in accordance with 18 U.S.C. Section 1734 solely to indicate this fact.

We would like to thank D. Barila, D. Flynn, and G. Salvesen for DNA constructs and Y.J. Cho for critical reading of the manuscript and helpful suggestions.

\section{References}

1. Yamaguchi H, Wyckoff J, Condeelis J. Cell migration in tumors. Curr Opin Cell Biol 2005; 17:559-64.

2. Helfer B, Boswell BC, Finlay D, et al. Caspase8 promotes cell motility and calpain activity under nonapoptotic conditions. Cancer Res 2006;66:4273-8.

3. Barnhart BC, Legembre P, Pietras E, Bubici C, Franzoso G, Peter ME. CD95 ligand induces motility and invasiveness of apoptosis-resistant tumor cells. EMBO J 2004;23:3175-85.

4. Stupack DG, Teitz T, Potter MD, et al. Potentiation of neuroblastoma metastasis by loss of caspase-8. Nature 2006;439:95-9.

5. Rossman KL, Der CJ, Sondek J. GEF means go: turning on RHO GTPases with guanine nucleotide-exchange factors. Nat Rev Mol Cell Biol 2005;6:167-80.

6. Zheng Y, Bagrodia S, Cerione RA. Activation of phosphoinositide 3-kinase activity by $\mathrm{Cdc} 42 \mathrm{Hs}$ binding to p85. J Biol Chem 1994:269:18727-30.
7. Innocenti M, Frittoli E, Ponzanelli I, et al. Phosphoinositide 3-kinase activates Rac by entering in a complex with Eps8, Abil, and Sos-1. J Cell Biol 2003;160:17-23. 8. Taniguchi CM, Aleman JO, Ueki K, et al. The p85 $\alpha$ regulatory subunit of phosphoinositide 3-kinase potentiates $\mathrm{c}$-Jun $\mathrm{N}$-terminal kinase-mediated insulin resistance. Mol Cell Biol 2007:27:2830-40.

9. Luo J. Cantley LC. The negative regulation of phosphoinositide 3-kinase signaling by p85 and it's implication in cancer. Cell Cycle 2005;4:1309-12.

10. Chang DW, Xing Z, Capacio VL, Peter ME, Yang X. Interdimer processing mechanism of procaspase-8 activation. EMBO J 2003;22:4132-42.

11. Cursi S, Rufini A, Stagni V, et al. Src kinase phosphorylates caspase-8 on Tyr380: a novel mechanism of apoptosis suppression. EMBO J 2006;25: 1895-905.

12. Rush J, Moritz A, Lee KA, et al. Immunoaffinity profiling of tyrosine phosphorylation in cancer cells. Nat Biotechnol 2005;23:94-101.
13. Boatright KM, Renatus $M$, Scott $F L$, et al. A unified model for apical caspase activation. Mol Cell 2003:1 1:529-41. 14. Fukata M, Nakagawa M, Kaibuchi K. Roles of Rhofamily GTPases in cell polarisation and directional migration. Curr Opin Cell Biol 2003;15:590-7.

15. Jimenez C, Portela RA, Mellado M, et al. Role of the PI3K regulatory subunit in the control of actin organization and cell migration. J Cell Biol 2000;151249-62.

16. Brachmann SM, Yballe CM, Innocenti M, et al. Role of phosphoinositide 3-kinase regulatory isoforms in development and actin rearrangement. Mol Cell Biol 2005;25:2593-606.

17. Hill KM, Huang Y, Yip SC, Yu J, Segall JE, Backer JM. $\mathrm{N}$-terminal domains of the class IA phosphoinositide 3kinase regulatory subunit play a role in cytoskeletal but not mitogenic signaling. J Biol Chem 2001;276:16374-8. 18. Sun T, Gao Y, Tan W, et al. A six-nucleotide insertiondeletion polymorphism in the CASP8 promoter is associated with susceptibility to multiple cancers. Nat Genet 2007:39:605-13 


\section{BROOKE M. HELFER}

4211 Summervale Dr., Murrysville PA 15668

- 724-263-0873-C

•helferbm@yahoo.com

\section{Education}

West Virginia University, Morgantown, West Virginia (current)

Ph.D. in Cancer Cell Biology, July 2008

Washington \& Jefferson College, Washington, Pennsylvania

B.A. in Cell/Molecular Biology and German, May 2004

- $\quad$ Study Abroad in Germany, January 2001

- Delta Phi Alpha member, German honorary

\section{Work Experience}

West Virginia University, Mary Babb Randolph Cancer Center, Morgantown WV, Fall 2004-present

Cancer cell biology program - doctoral candidate researcher

- $\quad$ Studied the non-apoptotic roles of caspase-8 and caspase-2

- Trained incoming graduate students

Washington \& Jefferson College, Genetics lab assistant, Washington, PA, Feb 2003May 2004

The Children's Hospital of Pittsburgh, Immunogenetics Lab, Pittsburgh, PA, Summers 2003, 2004

- Optimized 2D electrophoresis conditions for the separation of proteins in an insulin sensitive cell line

- Screened Western Blots of the cell line against diabetic patient serum samples

- Location of two putative autoantigens for Type 1 diabetes.

The Centers for Disease Control and Prevention, Atlanta, GA, Summer 2002

Viral and Rickettsial Zoonoses Branch - student researcher

Howard Hughes Medical Institute grant supported

- Performed DNA extraction, quantitation, and sequencing sample preparation

- Examined approximately 450 Ixodes scapularis ticks for the HGE agent

Washington \& Jefferson College, Mentor to incoming freshmen, Washington PA, Fall 2001, 2002

Washington \& Jefferson College, Biology Department, Washington, PA, Summer 2001 Howard Hughes Medical Institute grant supported

- Examined approximately 500 ticks for Lyme disease and HGE pathogens

- Found Anaplasma phagocytophila in Monroe County, PA, a novel finding

\section{Publications}

Helfer, B, J Senft, S Frisch. Caspase-8 interacts with the p85 subunit of phosphatidylinositol 3-kinase to regulate cell adhesion and motility. Cancer Rearch 2007; 67: 11505-11504

Helfer, B, B Boswell, D Finlay, A Cipres, K Vuori, T B Kang, D Wallach, A Dorfleutner, J Lahti, D Flynn, S Frisch. Caspase-8 promotes cell motility and calpain activity under nonapoptotic conditions. Cancer Research 2006; 66: 4273-4278 


\section{Presentations}

2007 E. J. Van Liere Convocation and Research Day, West Virginia University

- Caspase-2 Regulates Cell-Matrix Adhesion, April 2007

American Society of Cell Biology $\mathbf{4 6}^{\text {th }}$ Annual Meeting, San Diego, California

- Caspase-2 Regulates Cell-Matrix Adhesion, December 2006

2006 E. J. Van Liere Convocation and Research Day, West Virginia University

- Caspase 8 promotes cell motility under non-apoptotic conditions, April 28, 2006

$25^{\text {th }}$ Annual Undergraduate Symposium for Western Pennsylvania, Carlow College

- A Proteomic Approach for the Identification of Novel Autoantigens Related to Type 1 Diabetes, April 3, 2004

The Children's Hospital of Pittsburgh Summer Research Presentation

- A Proteomic Approach for the Identification of Novel Autoantigens Related to Type 1 Diabetes, August 7, 2003

$24^{\text {th }}$ Annual Undergraduate Biology Symposium for Western Pennsylvania, Washington and Jefferson College

- Human Granulocytic Ehrilchiosis Vectored by Ixodes scapularis: An Examination of Pennsylvania, Maryland, and New Jersey collected Ticks, April 12, 2003

Howard Hughes Medical Institute, External Advisory Board Presentation, Washington and Jefferson College

- Presentations of grant supported research, October 2001 and 2002 